Portland State University

PDXScholar

Spring 7-25-2013

\title{
Bicycle Traffic Count Factoring: An Examination of National, State and Locally Derived Daily Extrapolation Factors
}

Josh Frank Roll

Portland State University

Follow this and additional works at: https://pdxscholar.library.pdx.edu/open_access_etds

Part of the Sports Studies Commons, and the Urban Studies and Planning Commons Let us know how access to this document benefits you.

\section{Recommended Citation}

Roll, Josh Frank, "Bicycle Traffic Count Factoring: An Examination of National, State and Locally Derived Daily Extrapolation Factors" (2013). Dissertations and Theses. Paper 998.

https://doi.org/10.15760/etd.998

This Thesis is brought to you for free and open access. It has been accepted for inclusion in Dissertations and Theses by an authorized administrator of PDXScholar. Please contact us if we can make this document more accessible: pdxscholar@pdx.edu. 
Bicycle Traffic Count Factoring: An Examination of National, State and Locally Derived Daily Extrapolation Factors

by

Josh Frank Roll

A thesis submitted in partial fulfillment of the requirements for the degree of

\author{
Master of Urban Studies \\ in \\ Urban Studies \\ Thesis Committee: \\ Kelly Clifton, Chair \\ Chris Monsere \\ James Strathman
}

Portland State University

2013 


\begin{abstract}
Since nearly the beginning of the wide spread adoption of the automobile, motorized traffic data collection has occurred so that decision makers have information to plan the transportation system. Widespread motorized traffic data collection has allowed for estimating traffic volumes using developed extrapolation methods whereby short-term counts in sample locations can be expanded to longer periods. As states and local planning agencies make investments in bicycle infrastructure and count programs develop, similar extrapolation methods will be needed. The only available guidance on extrapolating bicycle counts comes from the National Bicycle and Pedestrian Documentation Project (NBPDP), yet no validation of these factors have been done to assess their usability in specific areas. Using bicycle traffic count data from the Central Lane Metropolitan Planning Organization Count Program in Oregon, this research demonstrates that using study area data to generate time-of-day factors. Factors are generated in two separate ways in order to reduce error from estimating daily bicycle volumes. Factors groups are developed using bicycle facility type where counts are collected. This research also seeks to add to the literature concerning bicycle travel patterns by using study area data to establish a university travel pattern exemplified by a flat hourly distribution from morning to evening.
\end{abstract}




\section{Table of Contents}

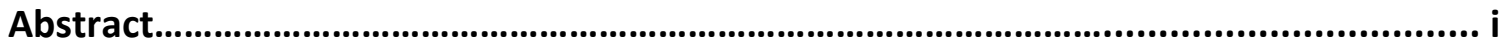

Chapter 1. Introduction ................................................................................................. 1

Chapter 2. Literature Review ......................................................................................... 11

Chapter 3. Data and Analysis........................................................................................... 21

3.1 -Introduction 21

3.2 - Comparison of Daily Factors from Available data Sources 21

3.2.1 Travel Survey Data Description $\quad 21$

3.2.2 Study Area Bicycle Traffic Count Data 22

3.3 - Creation of Time-of-Day Factors 30

3.4 - Establishing a "University" Bicycle Travel 34

3.5 - Validating the Application of Time-of-Day Factors 46

3.5.1 - Time-of-Day Factors from Study Area Data - Scenario 1

3.5.2 - Time-of-Day Factors from Study Area Data - Scenario 2

3.5.3 - Comparison of Scenario 1 and Scenario 2 Factor Results 97

\section{Discussion and}

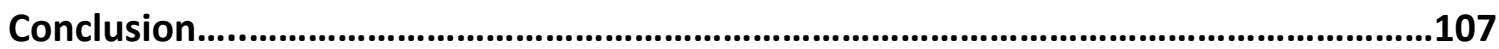

$\begin{array}{ll}\text { 4.1- Introduction } & 107\end{array}$

4.2- NHTS and OHAS Derived Factors 107

4.3 - Review of University Bicycle Travel Patterns 108

4.4 - Study Area Time-of-Day Factors Application 109

4.5 - Research Limitations $\quad 114$

$\begin{array}{ll}4.6 \text { - Conclusion } & 115\end{array}$

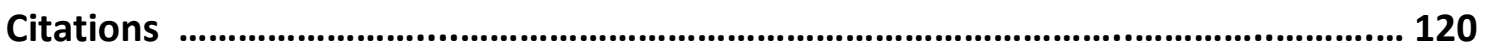

Appendices .....................................................................................................................125

Appendix A - Seasonal Comparison of Hourly Distributions 125

Appendix B - Proportion of Weekly Travel by Day of Week 134

Appendix C - All Daily and Hourly Counts 138

$\begin{array}{ll}\text { Appendix D - R Code } & 139\end{array}$ 


\section{List of Figures}

Figure 1.1 - Research Goals 5

Figure 1.2 - Map of Study Area $\quad 7$

Figure 1.3 - CLMPO Region Commute to Work by Bicycle Mode Share 8

Figure 2.1 - Traffic Factor Types $\quad 12$

Figure 3.1 - Count Station Locations for Study Area 26

Figure 3.2 - Seasonal Comparison of Hourly Distributions 27

Figure 3.3 - Proportion of Weekly Travel by Day of Week 29

Figure 3.4 - Hourly Distribution of Study Area Bicycle Traffic $\quad$ 40-42

Figure 3.5 - Examples of Bicycle Travel Patterns Using Study Area Data 43

Figure 3.6 - Characteristic of University Travel Pattern 45

Figure 3.7 - Time-of-Day Factoring Equation $\quad 47$

Figure 3.8 - Example of Application of Time-of-Day Factor 48

Figure 3.9 - Scenario 1 Time-of-Day Factor Creation Locations (20 locations) 52

Figure 3.10 - Scenario 1 Time-of-Day Factor Application Locations (20 locations) 53

Figure 3.11 - NBPDP Time-of-Day Factoring Equation $\quad 55$

Figure 3.12 - Summary of Application of Time-of-Day Factors for Scenario $1 \quad 56$

Figure 3.13 - Calculation of Measure of Error $\quad 57$

Figure 3.14 - Results for Scenario 1 Study Area Factors Compared to NBPDP Factors

Figure 3.15 - Iterative Process for Determining Most Effective Factors $\quad 73$

Figure 3.16 - Results for Scenario 2 Study Area Factors Compared to NBPDP Factors

Figure 4.1 - Annual Bicycle Traffic Estimation Equation 


\section{List of Tables}

Table 1.1 - U.S. Census Journey to Work by Bicycle Comparison 8

Table 1.2 - Bicycle Related Infrastructure Summary 9

Table 3.1 - 24-Hour Counts Collected by Season 23

Table 3.2 - 24-Hour Counts by Day of Week 23

Table 3.3 - Count Station Weather Summary $\quad 24$

Table 3.4 - Average Tuesday/Thursday Daily Comparison 24

Table 3.5 - Time-of-Day Am and PM Factor Comparison 32

Table 3.6 - Summary Statistics of Hourly Distributions 36

Table 3.7 - Range of Am and Pm Peak Factors for Locations Exhibiting University Travel Patterns $\quad 45$

Table 3.8 - Scenario 1 Time-of-Day Extrapolation Factors 49

Table 3.9 - Scenario 1 Factor Creation Location Daily Volumes $\quad 50$

Table 3.10 - Scenario 1 Factor Application Location Daily Volumes 51

Table 3.11 - Am Peak Factor Results using Study Area Factors $\quad 58$

Table 3.12 - Pm Peak Factor Results using Study Area Factors 60

Table 3.13 - Am Peak Factor Results using NBPDP Factors 62

Table 3.14 - Am Peak Factor Results using NBPDP Factors $\quad 64$

Table 3.15 - Exploring Effect of Outlier s- Average Absolute \% Difference Results $\begin{array}{ll}\text { Comparison } & \mathbf{6 8}\end{array}$

Table 3.16 - Average Absolute \% Difference Results Comparison by Factor Group

Table 3.17 - Relative Levels of Over-estimation

Table 3.18 - Scenario 2 Time-of-Day Extrapolation Factors $\quad 73$

Table 3.19 - Scenario 2 Am Peak Factor Results using Study Area Factors $\quad$ 75-78

Table 3.20 - Scenario 2 Pm Peak Factor Results using Study Area Factors $\quad$ 79-82

Table 3.21 - Scenario 2 Am Peak Factor Results using NBPDP Factors 83-86

Table 3.22 - Scenario 2 Pm Peak Factor Results using NBPDP Factors 87-90

Table 3.23- Relative Levels of Over-estimation $\quad 93$

Table 3.24- Exploring Effect of Outliers- Average Absolute \% Difference Results Comparison $\quad 94$

Table 3.25- Average Absolute Percentage Difference by Factor Group 94

Table 3.26 - Average Absolute Percentage Difference by Season 95

Table 3.27 - Average Absolute Percentage Difference by Tuesday/Thursday 95

Table 3.28 - Comparison of Scenario 1 and Scenario 2 Am Study Area Factors 98

Table 3.29 - Comparison of Scenario 1 and Scenario 2 Pm Study Area Factors 100

Table 3.30 - Comparison of Scenario 1 and Scenario 2 Am NBPDP Factors 102

Table 3.31 - Comparison of Scenario 1 and Scenario 2 Pm NBPDP Factors 104

Table 3.32 - Comparison of Scenario 1 and Scenario 2 Average Absolute Percentage Difference $\quad 106$

Table 4.1 - Annual Estimate of Bicycle Traffic Results 112 


\section{Introduction}

Motorized traffic data collection has been around nearly as long as the widespread adoption of the automobile. Understanding motorized traffic volumes is vital to planning the transportation system. As investments into infrastructure for nonmotorized traffic such as bicycles increase, similar dedication to tracking bicycle traffic is necessary. The literature suggests performance measures like bicycle counts be put in place to monitor the impact that investments into bicycle infrastructure are having on overall demand so that planners and policy makers can make more informed decisions (OAG 1994, OAG 1997, Fleisher, and Mahafy 1997). The use of short-term, typically two-hour peak period, manually-gathered bicycle counts is a popular method for collecting data on bicycle demand in many areas around the United States (FHWA 2005; FHWA 2011).

Short-term counts are not necessarily representative of demand beyond the brief count period when they are collected. However, these short term counts are oftentimes used to represent relative levels of traffic in comparison to one another and are also used to estimate demand for longer periods of time, such as daily, monthly or yearly. This is done by using short-term counts to estimate 24-hour counts, which in turn, are used to estimate longer term counts of interest (e.g. week, month, or year). Factors for expanding short-term counts to longer term counts are created by using 
patterns found in data from long term counts and applying them to data collected over a shorter period.

These longer-term estimates are more meaningful when trying to understand the related economic, health, safety and environmental effects of bicycle usage for research and planning. For instance, the World Health Organization (WHO) uses average daily bicycle travel to assess the health and economic impacts for new infrastructure or as inputs to larger health impact assessments (WHO 2011). A number of studies analyzing safety information utilize some form of daily counts (Wang and Nihan 2004, Sælensminde 2004). Further, travel demand modeling tools require an average daily figure to compare and validate estimated traffic volumes. As these analysis tools progress and bicycle travel is explicitly represented in these models, average daily bicycle counts will be needed for estimation and validation.

The National Bicycle and Pedestrian Documentation Project (NBPDP) have accumulated data from over 600 count locations, gathered by more than 100 organizations. These data have been used to build a spreadsheet tool that extrapolates short-term bicycle counts data such as two-hour manual counts, up to full day, week, monthly, and yearly estimates. The NBPDP is the only recognized standard in both data collection methodology and estimation for daily, weekly, monthly, and yearly counts from short-term data. The Seamless Travel Project (2010) claims the NBPDP data, "should be applicable to a broad range of communities nationwide". Some reports and 
studies (New Orleans 2009; Buffalo Valley 2012, FABB 2011, Alta/Land People 2010) have implemented the data collection and data extrapolation methodology. The World Health Organization cites the NBPDP as a resource for adjusting short-term counts to longer term levels of bicycling (WHO 2011). Currently no review has been published that examines the reliability of using the NBPDP extrapolation factors to estimate 24hour bicycle volumes.

This research will examine a number of items related to bicycle traffic factoring including a review of available data sets that describe bicycle traffic time-of-day travel patterns, exploration of bicycle travel patterns, and the formation and application of time-of-day extrapolation factors. The Central Lane Metropolitan Planning Organization (CLMPO) collects data via mobile automatic pneumatic tube counting devices. This data set includes bicycle traffic counts collected over multiple 24-hour periods across several months and locations which allows for the creation and application of a set of local extrapolation factors asd well as comparison of study area factors with factors derived from other sources.

The outcomes of this research will add to what is currently understood about bicycle traffic factoring in a number of ways. Firstly, a summary and comparison of existing data sources from which daily extrapolation factors could be derived will be presented. These existing data sources include both travel survey information and existing bicycle count data collected at the national, state and local level. This summary and 
comparison will establish the differences in morning and evening peak factors derived from these different data sets.

The second outcome of this research expands on the current literature regarding classification of bicycle traffic patterns. Bicycle traffic count data collected in the study area suggests that the current set of bicycle traffic patterns classifications should not be limited to commute and recreational (and mixes thereof) typologies as described in Miranda-Moreno 2013 but should include another category that describes bicycle travel related to university campuses. This "University" bicycle travel pattern is characterized by less distinguishable peaks in either the morning or evening and instead possesses a relatively stable traffic flow throughout the day. A higher numbers of bicyclists on average during weekdays compared to weekends is another element that characterizes 'University' bicycle travel patterns based on the data collected in the study region. The addition of this typology will be helpful to planners and practitioners looking to understand users on particular facilities but may not be as useful in the formation of time-of-day factor groups.

Lastly, the application of time-of-day extrapolation factors from study area bicycle counts and the NBPDP will be completed in order to give potential users of these factors some indication of their potential error. Using extrapolation factors that expand a short-term count to a full day 24-hour count from data collected in the study region, this work will assess the application of these factors in comparison to factors 
derived from national data sets gathered through the NBPDP. Currently, no published work has been done to assess the reliability of applying the NBPDP factors in specific areas of the U.S., leaving a gap in the understanding of how well these factors perform when employed. Understanding the accuracy of using these different factors will give planners and practitioners looking to expand short-term counts a better understanding regarding the possible levels of error. Figure $\mathbf{1 . 1}$ summarizes this research's primary goals.

Figure 1.1 - Research Goals

Research Goals
1. Summarize and compare daily expansion factors
derived from national, state and local data sources.
2. Use data collected in the study region to establish a
"University" bicycle traffic travel pattern.
3. Establish the reliability of commonly used national
daily expansion factors compared to locally derived daily
expansion factors.

The study area for this work is the CLMPO which encompasses the cities of Eugene, Springfield, and Coburg in the Willamette Valley of Oregon (See Figure 1.2 below) with a population of roughly 242,000 people. The region supports a strong bicycling culture making it an ideal location for this research. The U.S. Census shows that the EugeneSpringfield urbanized area consistently report a higher than the national average 
percentage of workers commuting to their jobs by bicycle (see Figure 1.3). U.S. Census information also (see Table 1.1) shows that Eugene has one of the highest percentages of bicycling to work mode shares in the country compared to other large bike-friendly communities, with $8.0 \%$ of workers commuting to work by bicycle (US Census 2010). The Springfield commute to work by bicycle percentage is $2.5 \%$ which is higher than the national average but lower than Eugene and the region as a whole. The CLMPO region supports 12 Safe Routes to School Programs with more schools in the region adding programs each year (McRhodes 2012). There are a number of advisory and advocate groups, including the Greater Eugene Area Riders (GEARS) group and Bike Lane Coalition, as well as Bicycle and Pedestrian Advisory Committees maintained by both Eugene and Springfield.

The region is home to the University of Oregon (UO) with a student population of approximately 25,000 . The campus has a strong effect on local bicycle traffic demand (CLMPO 2013) with a recent travel survey of students indicating that $26 \%$ use a bicycle as their primary means of transportation to school (UO 2013). The UO supports a number of bicycle friendly programs including the UO Bike Program that offers free space, guidance and tools to fix and maintain student bicycles. The UO has plans to initiate a bike share program very soon as well. Also, like many university towns throughout the country, a great deal of the bicycle traffic for the area is generated by the UO, since the campus has policies that restrict automobile travel to the campus. 
Figure 1.2 - Map of Study Area

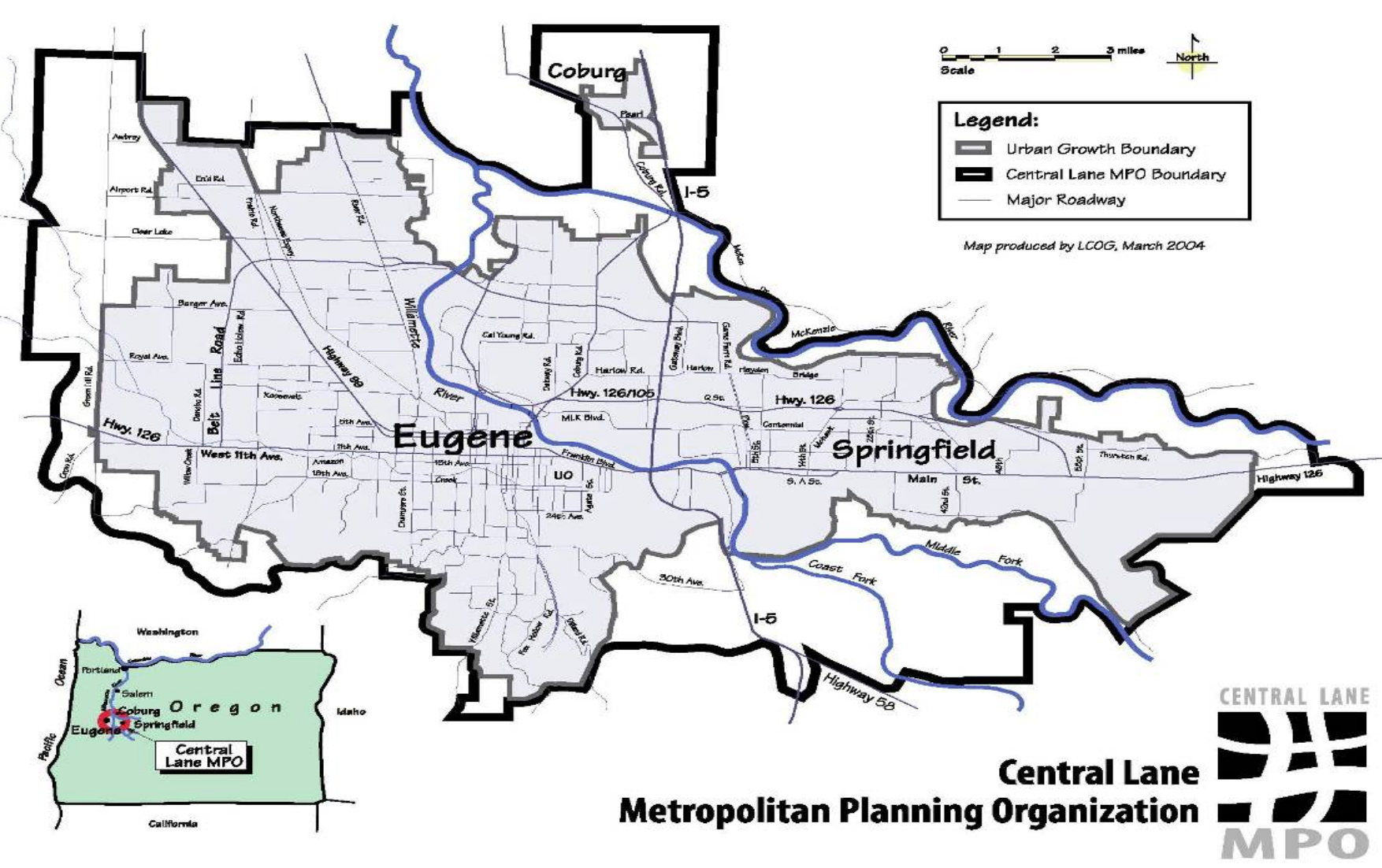


Figure 1.3 - CLMPO Region Commute to Work by Bicycle Mode Share

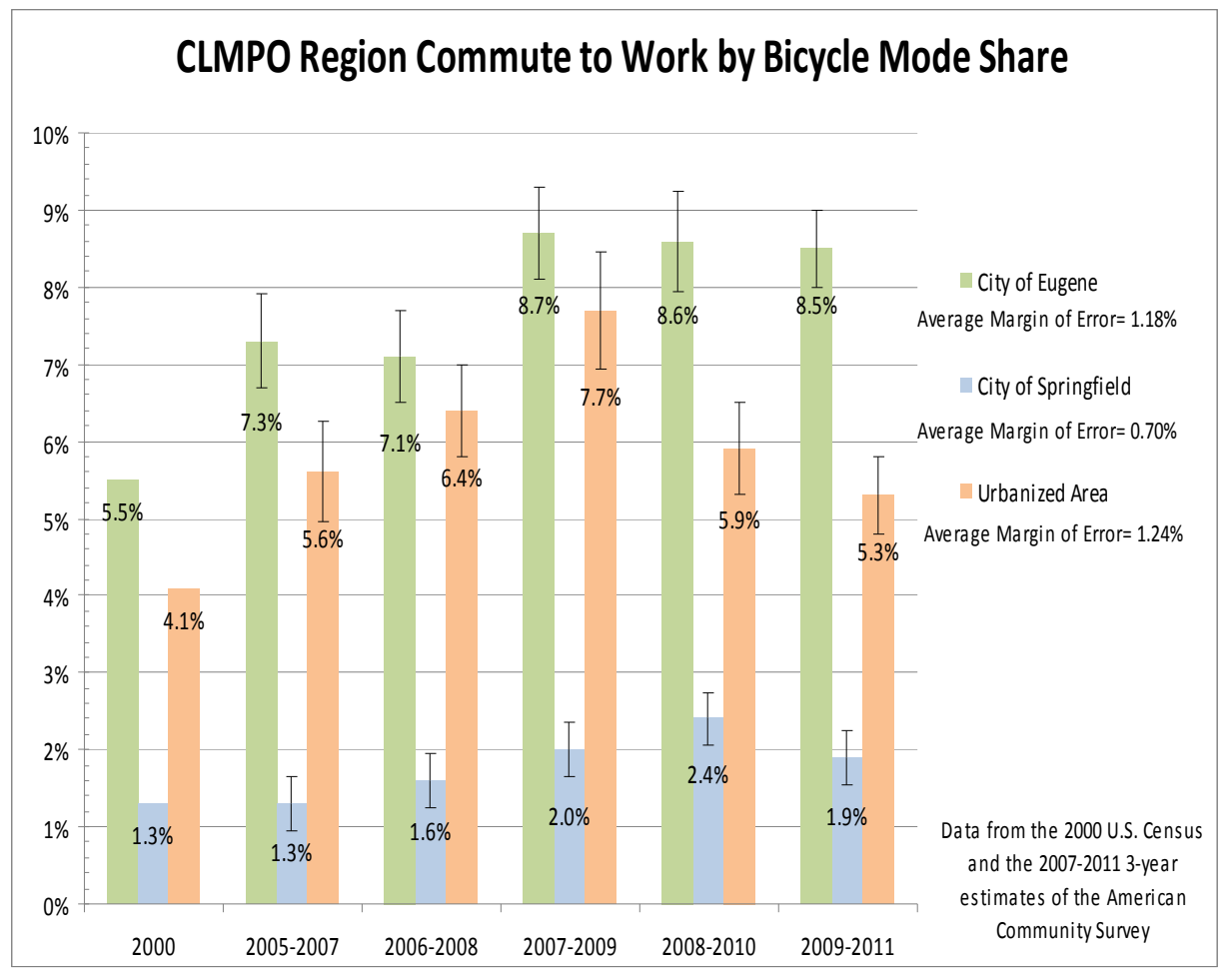

Table 1.1 - U.S. Census Journey to Work by Bicycle Comparison

\begin{tabular}{|c|c|c|c|}
\hline \multicolumn{4}{|c|}{2010 Census - Journey to Work \% } \\
\hline City & State & Population & $\begin{array}{l}\text { Percentage of } \\
\text { Bicycle } \\
\text { Commuters }\end{array}$ \\
\hline Davis & California & 65,740 & $22 \%$ \\
\hline Boulder & Colorado & 97,585 & $10 \%$ \\
\hline Eugene & Oregon & 156,299 & $8 \%$ \\
\hline Berkeley & California & 112,824 & $8 \%$ \\
\hline Cambridge & Massachusetts & 105,337 & $7 \%$ \\
\hline \multicolumn{2}{|c|}{ U.S. Average } & $306,738,433$ & $<1 \%$ \\
\hline
\end{tabular}


Additionally, the CLMPO region has extensive bicycle infrastructure with more than 120 miles of bike lanes, nearly 8 miles of bicycle boulevards, and a network of over 60 miles of off-street multiuse regional paths. Table 1.2 below details the number of miles of bicycle infrastructure by type and also describes attributes associated with each kind of infrastructure.

Table 1.2 - Bicycle Related Infrastructure Summary

\begin{tabular}{|c|c|c|}
\hline $\begin{array}{l}\text { Facility } \\
\text { Type }\end{array}$ & $\begin{array}{l}\text { Miles In CLMPO } \\
\text { network }\end{array}$ & Description \\
\hline Bike Lanes & 121 & $\begin{array}{l}\text { Bike Lanes are marked space along a length of } \\
\text { roadway designated for use by bicyclists. } \\
\text { Wheelchair users and some } \\
\text { motorized scooters are allowed in bike lanes. }\end{array}$ \\
\hline $\begin{array}{l}\text { Bike } \\
\text { Boulevard }\end{array}$ & 7.6 & $\begin{array}{l}\text { Low-volume and low-speed streets that have } \\
\text { been optimized for bicycle travel. Bicycle } \\
\text { Boulevard treatments can be applied at several } \\
\text { different intensities. The City will determine the } \\
\text { exact treatments needed for each corridor during } \\
\text { project design, but it is assumed that all Bicycle } \\
\text { Boulevards in Eugene will at a minimum have } \\
\text { way finding signs, pavement markings, traffic } \\
\text { calming (if needed to keep vehicle speeds low), } \\
\text { and some type of intersection crossing } \\
\text { treatments. }\end{array}$ \\
\hline $\begin{array}{c}\text { Multi-use } \\
\text { Regional } \\
\text { Path }\end{array}$ & 63 & $\begin{array}{l}\text { Multi-use regional paths are paved paths } \\
\text { separate from the roadway network that are } \\
\text { designed for both } \\
\text { walking and bicycling. Where space allows and if } \\
\text { sufficient additional maintenance funding can be } \\
\text { dedicated, an additional } \\
\text { unpaved path may be provided alongside the } \\
\text { paved path. }\end{array}$ \\
\hline No Facility & - & $\begin{array}{l}\text { A roadway that does not have any formal or } \\
\text { informal bicycle facilities. }\end{array}$ \\
\hline
\end{tabular}


Local planning priorities and policies also continue to support increasing current levels of bicycling. The region's local planning agencies have adopted goals to increase the bicycle mode share beyond its current level and have identified bicycle counts as one of the ways to monitor this progress (City of Eugene 2012, City of Springfield 1998).

The study area is located in the Marin West Coast climate zone with temperatures varying from cool to warm, with dry summers and cool wet winters. Some winter snowfall occurs but is often time irregular and typically does not accumulate in large quantities. 


\section{Literature Review}

The state of the practice for collecting traffic counts and developing factors from those counts for motorized travel is well established but remains in the development stage for bicycle traffic. Bike counts are becoming more common around the U.S. which has facilitated investigation into the development of factors for this mode. An important part of developing traffic expansion factors is definition of factor groups which are associated with the usage patterns at the count site. Some research attempts to use observed bicycle counts and their relationship with surrounding urban form variables to establish a method for using limited count data to estimate demand for larger geographic area. Much of this work is relatively new and so there is less accumulated knowledge on the subject.

An important example of advanced stage of motorized traffic monitoring is the Highway Performance Measuring System (HPMS). The HPMS is a national level highway information system that develops federal guidelines for state and local departments of transportation count collection programs (FHWA 2001). These guidelines are published in the Traffic Monitoring Guide (TGM) published by the Federal Highways Administration (FHWA) and details traffic evaluation protocols including data collection and processing (FHWA 2001). These data collection and processing guidelines inform state and local government transportation agencies on how to estimate total vehicle miles traveled (VMT) for their jurisdictions. The VMT estimates are important in 
understanding basic details about an area's transportation system and are significant metrics for allocating federal and state transportation funding dollars (MAP-21). A section of this guidance document details the creation and application of expansion factors that expand short-term counts of as little as 12 hours to daily, weekly, monthly, and annual estimates and also describe guidance as to the usage of factors for greater geographic coverage. Types of factors include:

Figure 2.1 - Traffic Factor Types

\begin{tabular}{|c|}
\hline $\begin{array}{l}\text { Factor Types } \\
\text { 1. Time-of-day: This factor adjusts a sub-daily count to a total } \\
\text { daily count. } \\
\text { 2. Day-of-week: This factor adjusts a single daily count to an } \\
\text { average weekday count, weekend count, or day of week count. } \\
\text { 3. Month/season-of-year: This factor adjusts an average daily } \\
\text { count to an average annual count. }\end{array}$ \\
\hline
\end{tabular}

Continuous counts (i.e., counts taken over the course of an entire year), which allow for a comprehensive understanding of day of the week, monthly, and other seasonal effects are used to construct the above described expansion factors. These adjustment factors are developed for distinct factor groups, which are groups of continuous counters with similar traffic patterns. The continuous counters in this factor group provide year-round traffic counts and permit short term counts to be annualized in a 
way to minimize error (TMG (2012). Counting programs are augmented by shorter term data collected systematically across a state and depending on resources, are bolstered by smaller agency data collection efforts at the county and city level.

The TMG tome has a soon to be released (July of 2013) chapter offering guidance on non-motorized traffic data collection and processing. Most of the principles used in motorized traffic counting apply to non-motorized traffic though there are some key differences. One of the major differences is the general state of the practice for nonmotorized data collection. Most non-motorized data collection programs are smaller scale and limited in duration, often times just two hours per day. The non-motorized section of the TMG describes the primary differences between motorized and nonmotorized count collection programs. An important difference is that non-motorized traffic is typically higher on roadways with fewer automobiles like shared use paths. Most motorized traffic counts are focused on streets with high volumes of automobiles. The last important difference between motorized and non-motorized traffic count programs according to the TMG, is that the technologies for counting bicycles and pedestrians are still emerging and the levels of error associated with these counting devices is not as well known. The forth-coming non-motorized section of the TMG offers guidance about creating factor groups from continuous data whereby patterns of variation are used to create groups of similar count locations that can be used to factor short-duration counts to annual volume estimates. Finally, the chapter on nonmotorized traffic counting in the TMG discusses data collection methods and 
technologies including referencing the National Bicycle and Pedestrian Documentation Project (Jones 2009) discussed in more detail below. More guidance is expected on non-motorized travel factoring through the National Cooperative Highway Research Project 8-78, which will be producing a guide for measuring bicycle and pedestrian use entitled "Estimating Bicycle and Pedestrian Demand for Planning and Project Development" to be completed June 2013 (TR News 2012).

Some progress has been made in the recent past to collect information from around the U.S. on non-motorized traffic. Jones (2009) attempts to establish a consistent national collection methodology and database for bicycle and pedestrian traffic counts. This methodology for count collection has become the current state of the practice and is also referenced in the forth-coming guidelines on non-motorized traffic monitoring (TMG 2012; Hankey et al. 2012). The Federal Highways Administration (FHWA) compiled information on count programs including methodologies and technologies which attempted to understand the state of the practice. A conclusion of this synthesis was that the common practice of extrapolating short-term sample counts has the "potential to produce skewed interpretations of the level of bicycling and or walking occurring in a community". The report also mentioned that, "the extrapolation of limited count locations to larger geographic areas is a common struggle and no known reliable solution exists" (FHWA 2011). The idea of using nationally derived factors can 
be questioned by findings in Jones (2009) which demonstrated that non-motorized traffic on trails in Indianapolis differ from patterns of use documented in other regions of the U.S. and concluded that, "unlike vehicle use patterns, there appear to be significant regional differences in seasonal patterns". There has been much less standardization in bicycle traffic factor estimation compared to methods long since developed for motorized traffic. For many years, at both the state and federal level, guidance has been issued for factoring automobile traffic but not for non-motorized transportation.

Even though much more work has been done in developing extrapolation factors for motorized traffic, similar work is beginning to be done for non-motorized traffic. Nordback et al. (2012) quantified the uncertainty when estimating annual average daily bicycle traffic counts from short-term counts. The authors use continuous count data from the equivalent of six permanent counting devices to form two sets of factors (one commute pattern and one non-commute pattern), and then tests the performance through application of those factors to different samples of short-term counts ranging from one hour peak period to four weeks. This research demonstrated that with short term counts of three hours or less, error could range from $25-58 \%$ absolute percentage difference, but with short term counts of at least a week, error could be minimized to an average of $22 \%$ absolute percertage difference. The research also concluded that to form meaningful factors, five to six continuous counters should be used. 
Defining meaningful factor groups is an important part of expansion factor development. For motorized traffic factor groups are typically defined by the federal classification of the road way, either a freeway/interstate, major/minor arterial, major or minor collector, or some other lower status facility type. These classifications are related to the total volumes observed on these facilities and make associating the usage patterns clear because of their connection with the observed traffic volumes. Bicycle traffic does not fit as nicely into this framework because of the variation in traffic volumes by facility type from one urban area to another (Barnes and Krizek 2005). Though facility type may not offer a high level definition of the usage patterns for a particular bicycle facility, Miranda-Moreno et al. (2013) offer another method for classifying usage patterns. Using data from five large North American cities including Montreal, Ottawa, Portland, San Francisco, and Vancouver, the authors create factor groups based on observed hourly distributions and total average weekday and weekend volumes. The factor groupings include a Primarily Utilitarian, Mixed-Utilitarian, MixedRecreational, and Primarily Recreational. These typologies represent the usage pattern on a continuum from strictly commuter with strong morning and evening and less travel on weekends compared to weekdays, to strictly recreational patterns where no definable morning or evening peaks periods exist but instead have a broad hourly peak from early afternoon to early mid evening hours with more daily travel on weekends compared to weekdays. These patterns are useful in helping to think about how to define factor groups for bicycle count stations, and the work from Miranda-Moreno et 
al. helps to inform one of the goals of this research which is to define another usage pattern discussed in more detail below.

Other work using bicycle count data include Lindsey et al. (2007) which advance methods using data collected through infrared detectors on urban greenway trails in Indianapolis, Indiana to develop two methods for estimating annual trail traffic. The first method used socio-demographic and urban form information in conjunction with time of year and weather attributes as inputs for a regression model to estimate annual trail traffic. This method provided estimates within $20-30 \%$ of the observed counts. The second method used a factoring methodology similar to the methodology proposed in this research where an observed sample of traffic is extrapolated to a full day estimate, which is then used to create week, month and annual estimates. This method produced results for monthly estimates ranging in accuracy from $-6.2 \%$ to $-31.6 \%$ and $20.2 \%$ to $-36.4 \%$ for annual traffic estimates. (Lindsey et al. 2007).

Similar to one of the methods featured in Lindsey et al. (2007), there are bicycle traffic estimation models that use socio-demographic and urban form information to predict bicycle volumes where count data has been collected. Hankey et al. (2012) developed an estimation model using bicycle counts data collected from 259 locations in Minneapolis, Minnesota using manual data collection methods. Using ordinary least squares and negative binomial regression the authors attempted to estimate 12-hour traffic counts using variables described in past studies as being relevant to bicycle traffic 
including weather, neighborhood socio-demographics, built environment characteristics, and road or bicycle facility type. The models developed were able to explain up to $47 \%$ of the variation using a negative binomial modeling approach. The results from Hankey et al. (2012) were derived by scaling (i.e., factoring or extrapolating) short-term two-hour counts to half-day counts (i.e., 12 hour counts) using a standard time-of-day factoring methodology but the authors do not appear to substantiate the reliability of this factoring. The analysis then uses these estimates of 12 -hour traffic to compare to the negative binomial and OLS models to test the accuracy of the 12-hour results. Comparing an estimate from one method to an estimate from another in order to assess the predictive capability of one method may lead to spurious conclusions.

Another associated area of research aims to estimate area wide bicycle demand using bicycle counts. For a study that attempts to create a bicycle and pedestrian demand model in San Diego County, researchers used data from two-hour manual counts from 80 locations in conjunction with land use and employment data in order to estimate weekday morning bicycle traffic. A number of different estimation approaches were tried using independent variables including the number of driveway approaches, number of sidewalks, total length of off-street paths, population density, and employment density within a quarter-mile of the count station. A stepwise method using the total length of off-street paths, and employment density was found to be the most reliable and was used to estimate bicycle travel patterns throughout the city of San Diego. (Caltrans 2010) 
Griuswold et al. (2011) predict bicycle intersection volumes by estimating a model using 2-hour counts at 81 locations. Using information about the intersections surrounding land use, transportation system, and site characteristics, the authors employed a log linear regression to estimate intersection bicycle volumes in Alameda County. No attempt was made at forecasting daily volumes of traffic.

Other studies attempt to estimate area wide bicycle demand using data sets that gauge different measures of bicycling such as responses from travel surveys and the U.S. Census commute to work survey. For example, Barnes and Krizek (2005) defined a simple model that uses data from the National Household Travel Survey and U.S. Census to estimate total daily adult bicyclist percentage for different geographic scales. The authors attempted to estimate this measure at the state, metropolitan statistical area (MSA) and disaggregate zone level for Minneapolis where data was available. Barnes and Krizek (2005) suggest that estimating bicycle travel from socio-demographic and land use characteristics is not likely to ever produce satisfactory results. They claim that because adjacent land uses do not necessarily generate demand for the facility, using adjacent land uses to predict demand is not always informative. This reality is related to the fact that not all bicycle travel is utilitarian, so relating an estimate of bicycle demand as a function of employment density or another similar measure, does not always hold theoretically. The authors also claim that when land use information is used to explain bicycle traffic it cannot account for the different levels of traffic in the same city. For instance, in Minneapolis one off-street path may exhibit four times as much traffic as an 
off-street path across town with similar surrounding land use characteristics. Barnes and Krizek (2005) continue by positing that the low levels of bicycling are causing sampling errors thus affecting estimation parameters for models using socio demographic and land use variables as inputs. And lastly, the authors suggest that, as noted by Car and Dill (2003) causation for bicycling and infrastructure go in the opposite direction, with a large number of bicyclists in an area offering the politically favorable conditions to build bicycle infrastructure. Any of these conditions make using sociodemographic and land use characteristics difficult to interpret in explaining bicycle demand.

While other research related to bicycle demand modeling has been done, there has not been a great deal of work published using bicycle count information as the basis for the factor estimations and a review of the error. No work has been done assessing the application of the NBPDP factors, though Jones' (2009) research has been cited frequently when different cities describe their bicycle count program methodology. 


\section{Data and Analysis}

\section{1 - Introduction}

The following section describes the data and analysis methodology used to address three primary research questions. The first goal of this research is to use available data sets to construct time-of-day expansion factors for comparison with one another and with those derived from study area bicycle count data. This research has access to travel survey information from the 2009 National Household Travel Survey (NHTS), the Oregon Household Activity Survey (OHAS), bicycle traffic count collected throughout the study region, information on factoring from the National Bicycle and Pedestrian Documentation Project (NBPDP), and bicycle count data collected throughout the study area. The second goal seeks to add to the existing literature related to bicycle traffic patterns by describing a "University" pattern using data collected from the study area. Lastly, using time-of-day factors developed from bicycle count data from the study area and the NBPDP, some validation tests will be conducted to determine levels of error associated with applying these factors. This validation analysis will answer the question for the study area: Are locally derived factors more sensible to use than those from the NBPDP?

\section{2 - Comparison of Daily Factors from Available Data Sources}

\subsection{1 - Travel Survey Data Description}


The NHTS data set used in this evaluation was collected in 2009 and is considered "the authoritative source of national data on the travel behavior of the American public." The dataset allows analysis of daily travel by all modes, including characteristics of the people traveling, their household, and their vehicles (NHTS 2009). The OHAS data set comes from a detailed study of 17,000 Oregon households collected between April 2009 and June 2012. Detailed information about travel behavior and sociodemographic information is gathered from each member of the surveyed households through the OHAS. These data will be used to inform travel related research for the entire state.

\subsection{2 - Study Area Bicycle Traffic Count Data}

The CLMPO Regional Bicycle Count Program began collecting data on bicycle traffic during the summer of 2012 in order to provide better information to local planners, policy makers, and the public about the effect of bicycle related infrastructure investments. Data collection began in July of 2012 with 20 sites surveyed using automatic tube counters capable of collecting 24-hour data for as many days as the device is deployed. Data has now been collected at over 50 different sites across the study region with varying number of days of data collection per site. Data collected in summer of 2012 was typically collected for 6 days or more but was limited to 20 count stations. Data collected in fall of 2012 and winter of 2013 focused on only Tuesday and Thursday due to the goals of the CLMPO RBCP (CLMPO 2012), and consists of 41-46 
locations. One goal of the RBCP is to count 48 regionally significant count stations each season, though due to device problems and vandalism not all 48 locations are successfully surveyed each season. Table 3.1 below describes the number of count performed in each season. Data collected in summer consists of multiple days, (between 6-10 days) while data collected in fall and winter adhere to the CLMPO RBCP protocol of collecting data on either a Tuesday and Thursday. Table 3.2 details the total number of 24-hour counts by day of the week. Total rainfall observed during deployment days (days when counting devices were recording bike traffic) and average temperature for deployment days are summarized in Table 3.3. Overall summer was warmer and drier than fall. Winter was colder and wetter than both summer and fall.

Table 3.1 - 24-Hour Counts Collected by Season

\begin{tabular}{|c|c|c|}
\hline \multicolumn{3}{|c|}{$\begin{array}{c}\text { Number of Sites Counted Each } \\
\text { Season }\end{array}$} \\
\hline $\begin{array}{l}\text { Summer } \\
2012\end{array}$ & $\begin{array}{l}\text { Fall } \\
2012\end{array}$ & $\begin{array}{l}\text { Winter } \\
2013\end{array}$ \\
\hline 20 & 46 & 41 \\
\hline
\end{tabular}

Table 3.2 -24-Hour Counts by Day of Week

\begin{tabular}{|c|c|}
\hline \multicolumn{2}{|c|}{ 24-Hour Counts by Day of Week } \\
\hline Day of week & 24 Hour counts \\
\hline Sunday & 52 \\
\hline Monday & 37 \\
\hline Tuesday & 87 \\
\hline Wednesday & 38 \\
\hline Thursday & 90 \\
\hline Friday & 43 \\
\hline Saturday & 52 \\
\hline Total & 399 \\
\hline
\end{tabular}


Table 3.3 - Count Station Weather Summary

\begin{tabular}{|c|c|c|c|}
\hline \multicolumn{4}{|c|}{ Weather Information on Deployment Days By } \\
Season
\end{tabular}

These weather differences combined with fewer students on cmapus in summer result in lower expected average daily traffic values by season represented in Table 3.4 below. For count sites where counts were collected during each season, the differences in total daily counts can be observed. Not unexpectedly, bicycle traffic increases from summer into fall but then drops off again once the rain and cold of winter arrive.

Table 3.4 - Average Tuesday/Thursday Daily Comparison

\begin{tabular}{|c|c|c|c|}
\hline \multicolumn{4}{|c|}{ Count Data Average Weekday (Tuesday-Thursday) } \\
\hline Count Location & $\begin{array}{l}\text { Summer } \\
2012\end{array}$ & $\begin{array}{l}\text { Fall } \\
2012\end{array}$ & $\begin{array}{l}\text { Winter } \\
2013\end{array}$ \\
\hline 12th West High & 365 & 466 & 393 \\
\hline 15th East Agate & 321 & 1021 & 814 \\
\hline 15th West Alder & 462 & 974 & 579 \\
\hline Alder South 18th & 1215 & 1862 & 1085 \\
\hline 13th West Alder & 1298 & 2602 & 1631 \\
\hline 18th West Alder & 348 & 646 & 248 \\
\hline Agate South Franklin & 531 & 576 & 337 \\
\hline Onyx North Franklin & 662 & 1121 & 707 \\
\hline $\begin{array}{l}\text { DeFazio South River East } \\
\text { Bridge }\end{array}$ & 713 & 566 & 230 \\
\hline Frohnmayer South River & 1142 & 1691 & 637 \\
\hline
\end{tabular}


The map in Figure 3.1 describes the count locations throughout the study area over the three different seasons. 
Figure 3.1 - Count Station Locations for Study Area

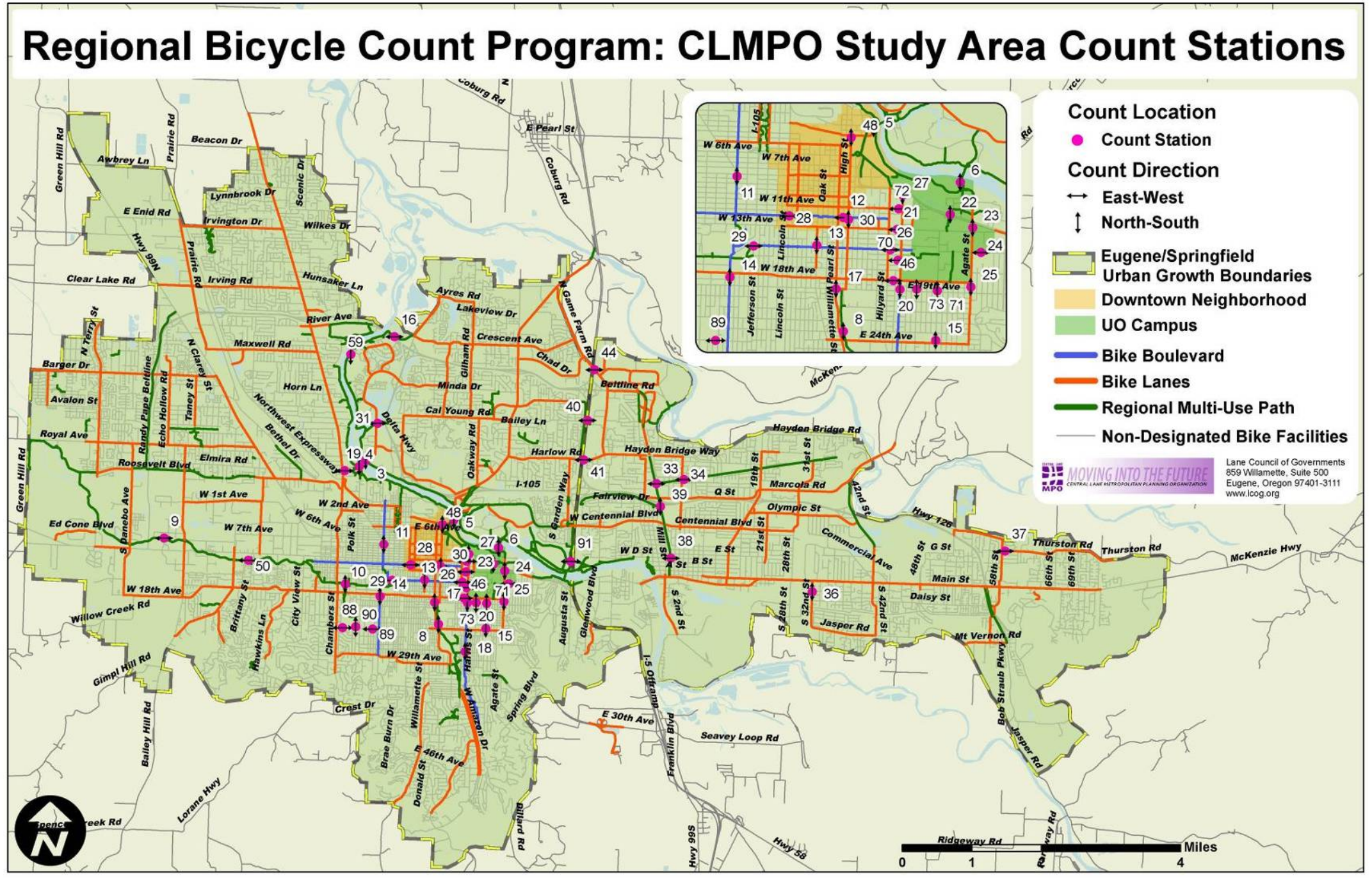


Even though average daily traffic data collected in the study region varies across different seasons, hourly distributions remain relatively stable for most of the locations surveyed. Figure 3.2 below demonstrates the hourly distributions for a subset of count stations where data made comparisons possible, and additional comparisons are available in Appendix A. These plots represent data collected on either a Tuesday or a Thursday and have the total average daily traffic for each season presented in the right hand margin of each plot for context.

Figure 3.2 - Seasonal Comparison of Hourly Distributions
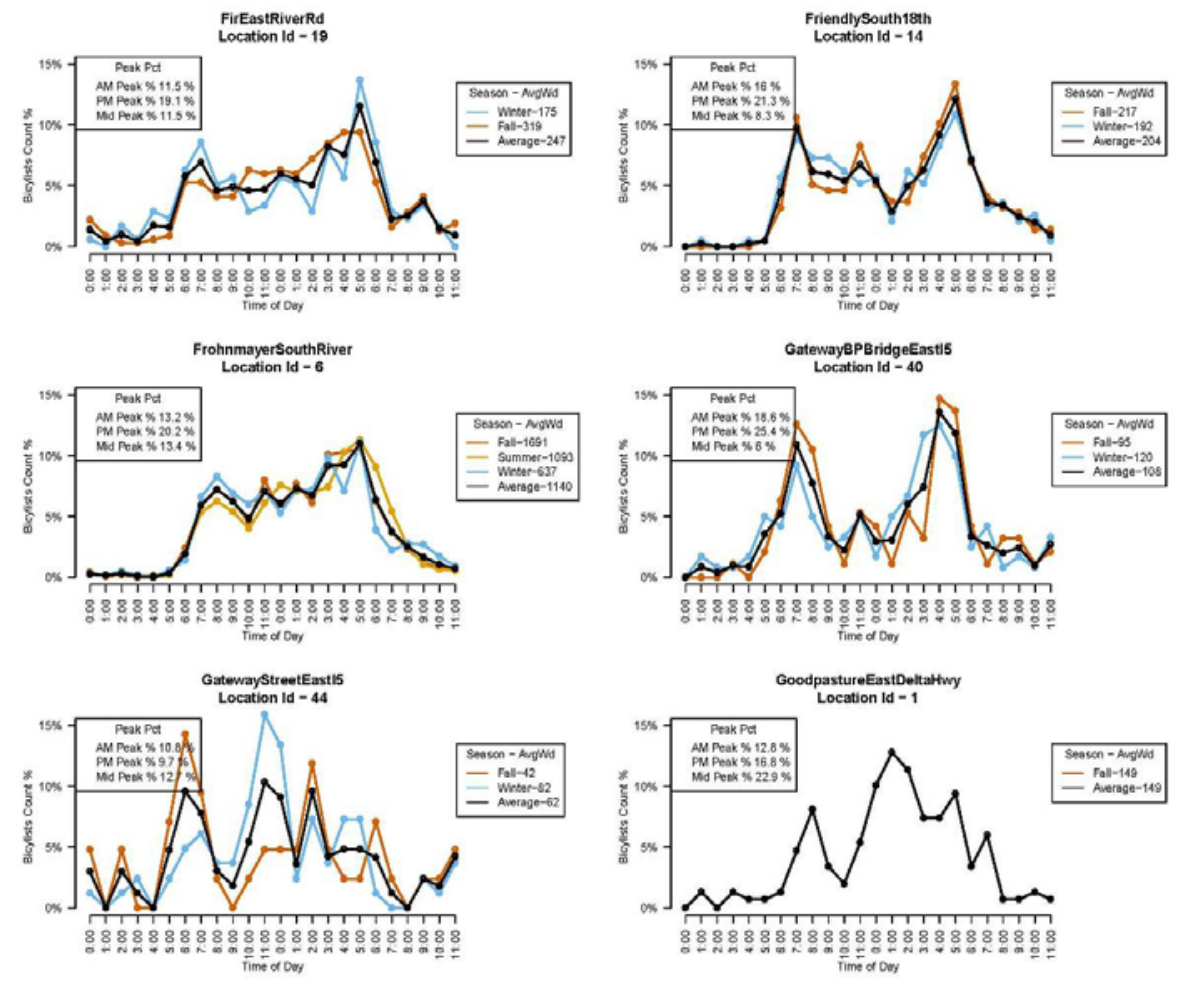

Due to the structure of the data collection program Tuesday and Thursday are the typical days when data is collected. However, for data collected in the summer of 2012, 
counting devices were deployed for longer periods of up to a week or more. Figure 3.3 provides a summary of these summer data by normalizing each daily traffic summary to the weekly average in order to demonstrate the relative daily traffic by day of the week. This summary also presents the average weekday traffic compared to the average weekend traffic. These pieces of information are informative and allow for some idea of what kinds of users are using the facility. Some days do not have a bar representing the proportion of weekly traffic because no data was collected on that day. Figure 3.3 summarizes four locations' weekday proportions with additional locations weekday proportions presented in Appendix B. Since most locations where multiple days of data were collected are near the University of Oregon, the daily proportions are higher for weekdays compared to weekends. The above data summaries are presented in order to describe the count data collected in the study region. All data including daily and hourly summaries can be found in Appendix C. 
Figure 3.3 - Proportion of Weekly Travel by Day of Week

11thWestAlder Average Daily Factors for July

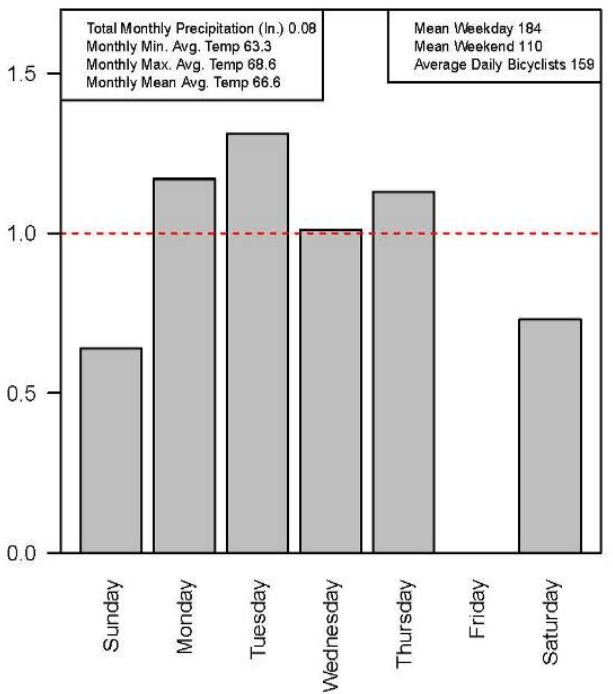

12thWestHigh Average Daily Factors for September

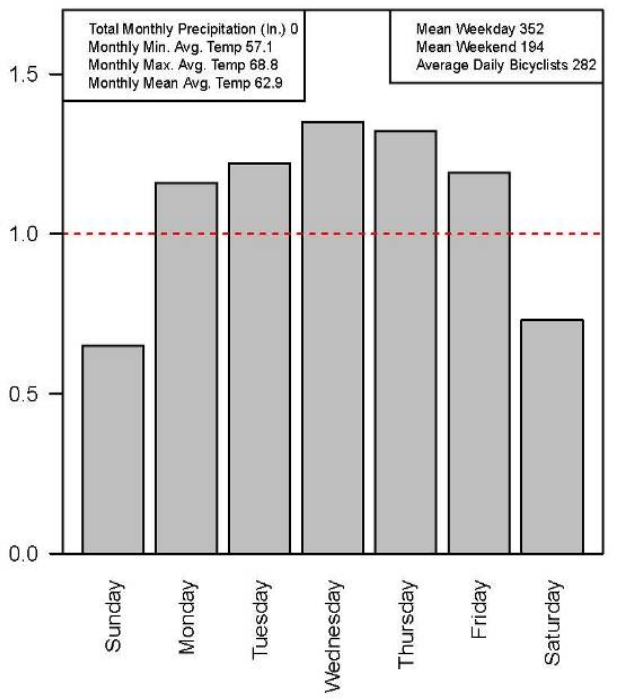

12thEastLincoln Average Daily Factors for September

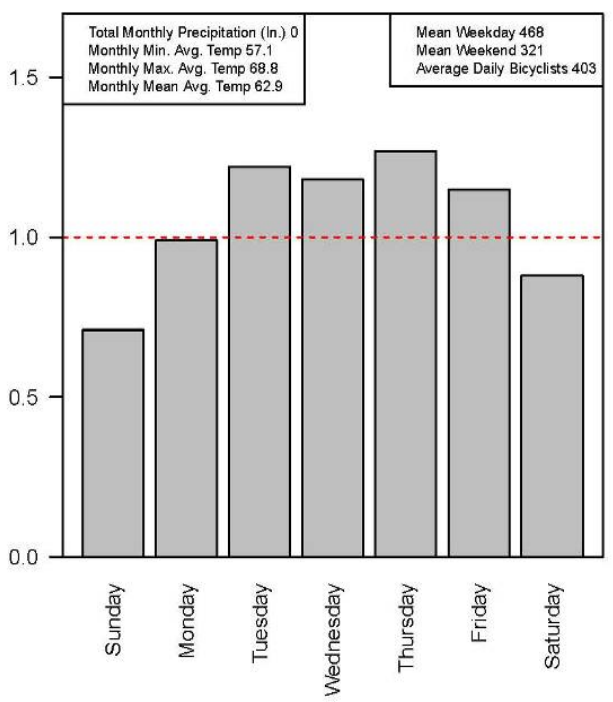

13thWestAlder Average Daily Factors for July

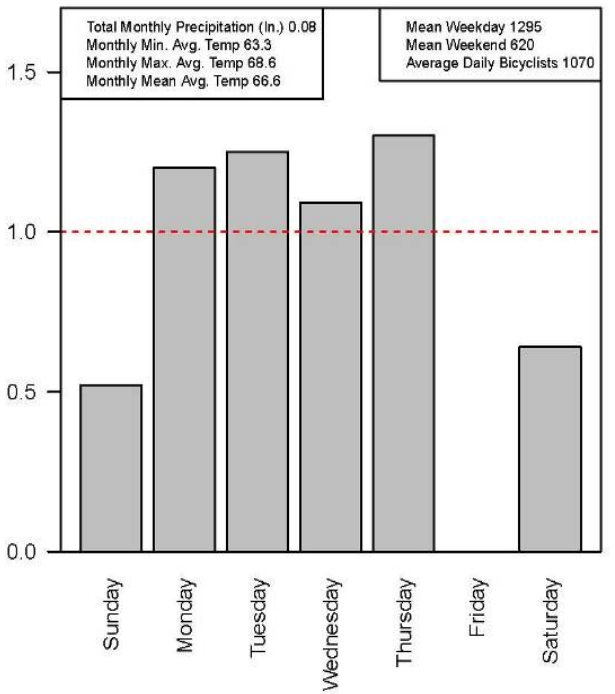




\subsection{Creation of Time-of-Day Factors}

As described in the Literature Review section, a time-of-day factor adjusts a subdaily (typically two-hour) count to a total daily count estimate. For example, a common practice for many bike count programs is to count during the morning or evening peak periods, so a time-of-day factor could be employed to extrapolate the counts collected for 2-hours into a total daily estimate. The NHTS and OHAS data sets are both travel surveys with detailed information collected from a sample of Americans about their daily transportation choices, including when they start their trips. In order to construct the factors in the table below, trip arrival time was summarized to the hour in which the trip took place for all trip purposes. There is some geographic resolution to the NHTS since some places could request over sampling in their area in exchange for funding to do so, but the data summarized below represents all households in the data set regardless of location, therefore representing the U.S. average. Factors constructed from OHAS data account for the city in Oregon where surveys were completed and also summarize the state overall. It should be noted that the OHAS data summarized below were done using household weights applied to the trips data where person weights would have been more appropriate. The Oregon Department of Transportation identified issues with the person weights so reformation of these weights is in progress at this time. 
The factors constructed from bicycle traffic counts collected in the study region were derived in two ways with each set of results presented in the table. One of the goals of this research is to establish reliable time-of-day factors; therefore two different factor sets have been constructed. The factors in Scenario 1 were created by averaging the AM and PM peak period percentages from data collected in the summer of 2012 by factor groups based on the bicycle facility type in which the counts were collected. Described in more detail in section 3.5.1, factors from Scenario 1 will be applied to independent data collected in the fall of 2012 to determine the levels of error associated with the their application. Also described in more detail below, the factors in Scenario 2 are derived from an iterative process where different factors were tested against all available study area data to determine which factors are best at minimizing error of estimated daily counts. It was found that another factor group should be created to account for differences in bicycle traffic patterns on path facilities. It should also be pointed out that these factors are constructed using data from Tuesday and Thursdays only, therefore minimizing some of the variation that may exist in hourly distributions had all days of the week been used.

Lastly, time-of-day factors derived from the NBPDP data are also presented in the table below. These factors are the factors used in a section 3.5.1 and 3.5.2 of this research and are derived from the NBPDP spreadsheet tool developed to extrapolate short-term counts to longer term estimates. Using the data described above, time-of- 
day factors have been constructed and are presented below for comparison. These factors are presented in Table 3.5 below.

Table 3.5 - Time-of-Day AM and PM Factor Comparison

\begin{tabular}{|c|c|c|c|c|}
\hline \multicolumn{5}{|c|}{ Time-of-Day Factor Comparison } \\
\hline \multicolumn{2}{|c|}{ Data set } & $\begin{array}{c}\text { AM Peak } \\
\text { Factor (7-9 AM) }\end{array}$ & $\begin{array}{l}\text { PM Peak } \\
\text { Factor (4- } \\
6)\end{array}$ & $\begin{array}{c}\text { Observati } \\
\text { ons in } \\
\text { Survey }\end{array}$ \\
\hline \multicolumn{2}{|c|}{ NHTS } & 0.11 & 0.20 & 9443 \\
\hline \multirow[b]{5}{*}{ OHAS } & Oregon & 0.20 & 0.20 & 3564 \\
\hline & Eugene & 0.21 & 0.19 & 666 \\
\hline & Springfield & 0.06 & 0.14 & 42 \\
\hline & Portland & 0.21 & 0.21 & 1151 \\
\hline & Bend & 0.19 & 0.21 & 226 \\
\hline \multirow{4}{*}{$\begin{array}{c}\text { Study Area Counts } \\
\text { - Scenario } 1\end{array}$} & Path & 0.11 & 0.21 & NA \\
\hline & Lane & 0.13 & 0.16 & NA \\
\hline & Blvd & 0.13 & 0.18 & NA \\
\hline & No Facility & 0.13 & 0.17 & NA \\
\hline \multirow{5}{*}{$\begin{array}{c}\text { Study Area Counts } \\
\text { - Scenario } 2\end{array}$} & Path - Rec & 0.15 & 0.25 & NA \\
\hline & Path - Commute & 0.13 & 0.21 & NA \\
\hline & Lane & 0.15 & 0.17 & NA \\
\hline & Blvd & 0.15 & 0.19 & NA \\
\hline & No Facility & 0.13 & 0.19 & NA \\
\hline \multicolumn{2}{|c|}{ NPBDP Counts } & 0.10 & 0.15 & NA \\
\hline
\end{tabular}

As noted above, the NHTS and OHAS factors are derived from trip data for all trip purposes. NHTS data looks a bit more reasonable with the AM peak being smaller than the PM peak which is what would be expected based on observations from data collected in the study area. This observation does not apply to some of the OHAS factors however, where each set of factors by city, including the state as a whole, demonstrate nearly identical factors for each peak period. Springfield is the only subset 
of OHAS data that adheres to the expected result with a higher evening peak relative to the morning peak though the number of observations was minimal for this subset. The use of household weights instead of the more appropriate person level weights may be playing a role in the similarity between AM and PMm peak factors. The study area factors offer a more detailed summary of factors as they have been assigned factor groups based on bicycle facility type (for Scenario 1 ) and a bicycle facility type and likely user type classification (Scenario 2). When applied in this way, the time-of-day factors base on these factor group specifications yield less error when deployed as time-of-day factors as demonstrated in a later section below. Each set of factors from the study area see larger factors (indicating higher percentage of daily traffic) in the morning compared with the evening which suggests that recreational and other non-work related bicycle travel is occurring. And lastly, the NBPDP factors are consistent with what would be expected with more travel in the PM compared to the AM as reflected in a larger factor for the evening peak as compared to the morning peak.

The NHTS derived factors are similar to some of the factors derived from study area data and the trip information does give some details about trips purpose but without more information about the facility the trip took place on, deriving more detailed factor groups is impossible. The OHAS data does not line up well with factors derived from study area either and would likely be an unreliable source to base time-of-day factors on. Validation of the study area factors will take place in section 3.5 .1 and 3.5.2 below. 


\section{4 - Establishing a "University" Bicycle Traffic Pattern}

From a set of continuous count data from several North American cites, MirandaMoreno (2013) dissect bicycle traffic patterns to create factor groupings based on usage patterns utilizing observed hourly distribution patterns and weekday versus weekend average daily counts. Data collected in the study region suggest consideration of another bicycle traffic pattern related to university travel known as, a "University" travel pattern. Unlike the data used in Miranda-Moreno (2013) which was from multiple continuous counters, data from the study region was collected with temporary tube counters and is therefore limited to at most to 10 days of continuously collected data. Additionally, for a majority of the data, only single day snap shots have been collected, representing either a Tuesday or Thursday. Because of these limitations, definition of a university travel pattern will be limited to exploration only and more data collection will be needed for a firmer classification.

Reviewing the hourly distributions for count stations in the study area, some consistencies are observable that could be useful in classifying certain locations as exhibiting a university travel pattern. The plots in Figure $\mathbf{3 . 4}$ below detail the hourly distributions for a subset of count locations in the study region and display summary statistics that help to establish the university travel pattern. For many of the locations, a generally flat hourly distribution can be observed between the hours of 7 AM and 6 PM. A standard deviation calculation of the hourly percentages between $7 \mathrm{AM}$ and 6 
PM is less than .02 for many of the locations near the university. A comparison of this "uniformity" metric (across all count stations where data is available) demonstrates a consistent finding where locations near the university have a uniformity metric of less than 0.02. Table 3.5 details this uniformity metric (StdDev7-6) as well as the AM, PM, and Mid-day (12 PM-2 PM) factors. 
Table 3.6 - Summary Statistics of Hourly Distributions

\begin{tabular}{|c|c|c|c|c|c|}
\hline Location & $\begin{array}{l}\text { AM } \\
\text { Factor }\end{array}$ & $\begin{array}{l}\text { PM } \\
\text { Factor }\end{array}$ & $\begin{array}{l}\text { Mid } \\
\text { Factor }\end{array}$ & $\begin{array}{l}\text { StdDev7-6 } \\
\text { (Uniformity } \\
\text { Metric) }\end{array}$ & $\begin{array}{l}\text { Near } \\
\text { University }\end{array}$ \\
\hline HighSouth4th & 0.126 & 0.15 & 0.15 & 0.012 & FALSE \\
\hline 12thEastLincoln & 0.119 & 0.162 & 0.162 & 0.014 & TRUE \\
\hline 15thEastAgate & 0.127 & 0.146 & 0.146 & 0.014 & TRUE \\
\hline 18thWestAlder & 0.107 & 0.153 & 0.153 & 0.014 & TRUE \\
\hline 13thWestAlder & 0.137 & 0.132 & 0.132 & 0.015 & TRUE \\
\hline 15thWestAlder & 0.139 & 0.174 & 0.174 & 0.016 & TRUE \\
\hline 16thWestAlder & 0.11 & 0.144 & 0.144 & 0.016 & TRUE \\
\hline 12thEastLawrence & 0.153 & 0.158 & 0.158 & 0.017 & TRUE \\
\hline AgateSouthFranklin & 0.148 & 0.188 & 0.188 & 0.017 & TRUE \\
\hline AlderSouthFranklin & 0.184 & 0.155 & 0.155 & 0.017 & TRUE \\
\hline OnyxNorthFranklin & 0.153 & 0.186 & 0.186 & 0.017 & TRUE \\
\hline PearlSouth19th & 0.093 & 0.176 & 0.176 & 0.017 & TRUE \\
\hline 24thEastFilmore & 0.163 & 0.156 & 0.156 & 0.018 & FALSE \\
\hline FrohnmayerSouthRiver & 0.132 & 0.202 & 0.202 & 0.018 & TRUE \\
\hline FirEastRiverRd & 0.094 & 0.188 & 0.188 & 0.019 & FALSE \\
\hline UniversitySouth18th & 0.096 & 0.19 & 0.19 & 0.019 & TRUE \\
\hline DefazioSouthRiverEastBridge & 0.132 & 0.201 & 0.201 & 0.02 & FALSE \\
\hline HighNorth13th & 0.206 & 0.127 & 0.127 & 0.02 & TRUE \\
\hline PolkNorth24th & 0.168 & 0.145 & 0.145 & 0.02 & FALSE \\
\hline MonroeSouth8th & 0.114 & 0.194 & 0.194 & 0.021 & FALSE \\
\hline WillametteNorth15th & 0.059 & 0.153 & 0.153 & 0.021 & FALSE \\
\hline FernRidgeWestChambers & 0.137 & 0.201 & 0.201 & 0.022 & FALSE \\
\hline 24thWestAdams & 0.142 & 0.172 & 0.172 & 0.023 & FALSE \\
\hline UniversitySouth24th & 0.128 & 0.18 & 0.18 & 0.023 & TRUE \\
\hline
\end{tabular}




\begin{tabular}{|c|c|c|c|c|c|}
\hline 11thWestAlder & 0.085 & 0.207 & 0.207 & 0.025 & TRUE \\
\hline 12thWestHigh & 0.089 & 0.215 & 0.215 & 0.025 & TRUE \\
\hline GWestMohawk & 0.126 & 0.118 & 0.118 & 0.025 & FALSE \\
\hline AlderNorth27th & 0.17 & 0.188 & 0.188 & 0.026 & FALSE \\
\hline FriendlySouth18th & 0.16 & 0.214 & 0.214 & 0.026 & FALSE \\
\hline HarlowEastl5 & 0.14 & 0.162 & 0.162 & 0.026 & FALSE \\
\hline HeronBridgeSouthFernRidge & 0.156 & 0.204 & 0.204 & 0.026 & FALSE \\
\hline 15thWestJefferson & 0.111 & 0.228 & 0.228 & 0.027 & FALSE \\
\hline 32ndSouthOregon & 0.144 & 0.192 & 0.192 & 0.027 & FALSE \\
\hline SouthbankSouthGreenwayBr & 0.126 & 0.224 & 0.224 & 0.027 & FALSE \\
\hline AmazonPathNorth24th & 0.196 & 0.195 & 0.195 & 0.029 & FALSE \\
\hline GatewayStreetEastl5 & 0.108 & 0.097 & 0.097 & 0.029 & FALSE \\
\hline RichardsonBridge & 0.169 & 0.242 & 0.242 & 0.029 & FALSE \\
\hline NorthbankEastKnickerbocker & 0.103 & 0.233 & 0.233 & 0.03 & FALSE \\
\hline NorthbankWestDeltaHwy & 0.072 & 0.215 & 0.215 & 0.03 & FALSE \\
\hline DEastPioneerPkwyPath & 0.128 & 0.242 & 0.242 & 0.031 & FALSE \\
\hline HarrisSouth18th & 0.091 & 0.182 & 0.182 & 0.032 & FALSE \\
\hline AgateSouth18th & 0.1 & 0.23 & 0.23 & 0.034 & TRUE \\
\hline GoodpastureEastDeltaHwy & 0.128 & 0.168 & 0.168 & 0.034 & FALSE \\
\hline UniversityNorth24th & 0.105 & 0.194 & 0.194 & 0.035 & TRUE \\
\hline 42ndNorthCommercial & 0.105 & 0.186 & 0.186 & 0.036 & FALSE \\
\hline FernRidgeEastDanebo & 0.177 & 0.27 & 0.27 & 0.036 & FALSE \\
\hline NorthbankSouthOwossoBr & 0.105 & 0.267 & 0.267 & 0.037 & FALSE \\
\hline EWEBPathEastPioneerPkwy & 0.192 & 0.256 & 0.256 & 0.038 & FALSE \\
\hline NorthbankSouthGreenwayBr & 0.102 & 0.283 & 0.283 & 0.038 & FALSE \\
\hline GatewayBPBridgeEastl5 & 0.186 & 0.254 & 0.254 & 0.039 & FALSE \\
\hline PioneerPkwySouthQ & 0.088 & 0.198 & 0.198 & 0.039 & FALSE \\
\hline ThurstonEast58th & 0.226 & 0.14 & 0.14 & 0.04 & FALSE \\
\hline DeltaBPBridgeEastGoodPasture & 0.126 & 0.273 & 0.273 & 0.041 & FALSE \\
\hline EWEBPathEast5th & 0.197 & 0.184 & 0.184 & 0.041 & FALSE \\
\hline
\end{tabular}


The uniformity metric is good at highlighting locations with relatively flat hourly distributions but does not always point out locations near the university, as three locations with a less than 0.02 uniformity metric are not very close to the university. This might indicate that locations not near the university still experience university related travel, or that the flatness in the hourly distribution is not a good indicator of university related travel. Since 13 of the 15 locations where the uniformity metric indicates a university travel pattern are near the university, the former supposition might be more correct. 
Figure3.4- Hourly Distribution of Study Area Biycle Traffic

11thWestAlder

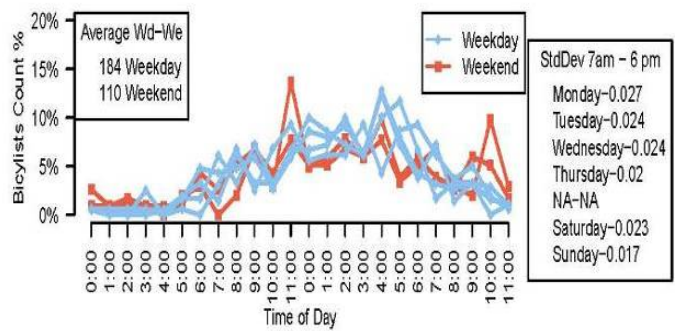

12thWesthigh

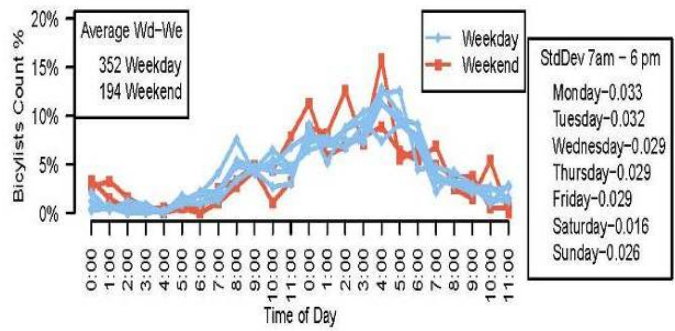

15thEastAgate

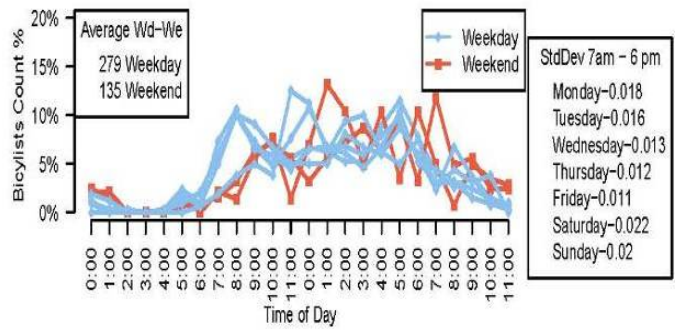

16thWestAlder

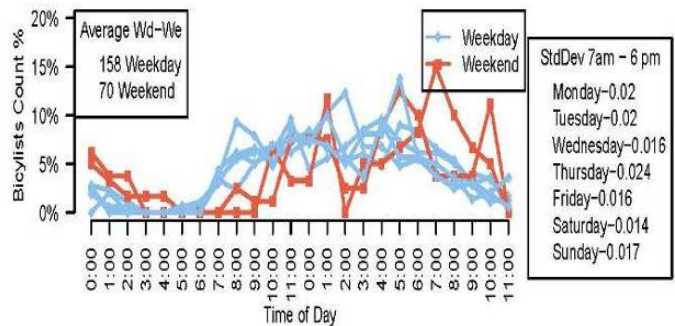

12thEastLincoln

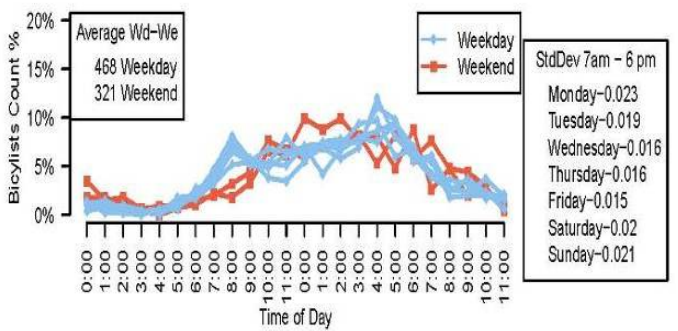

13thWestAlder

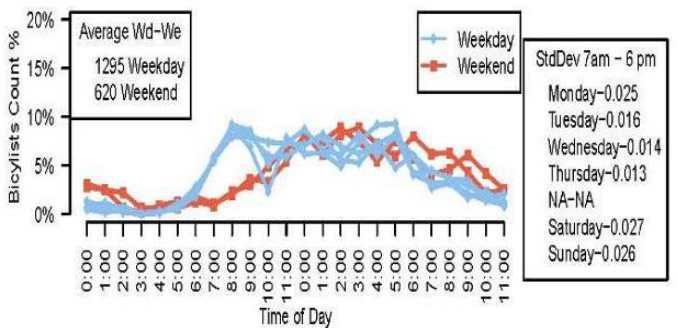

15thWestAlder

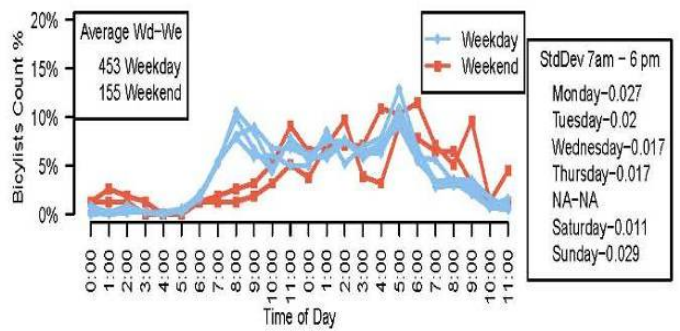

18thWestAlder

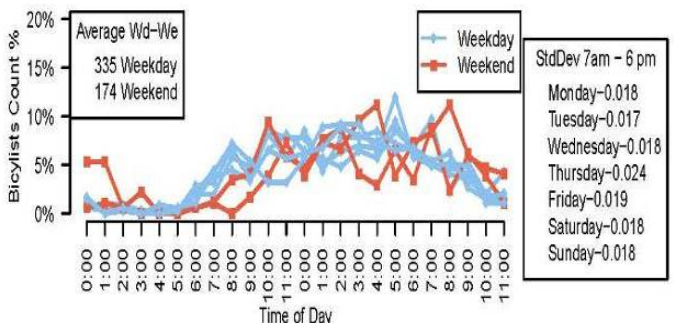


24thEastFilmore

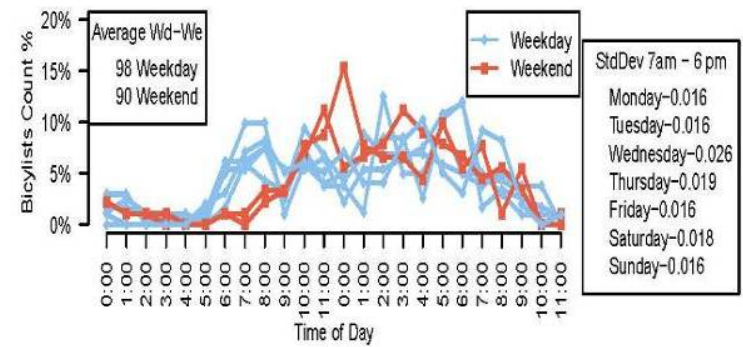

AgateSouth18th

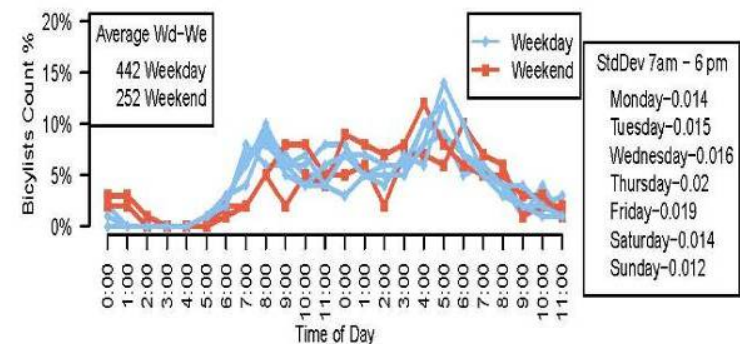

AlderSouthFranklin

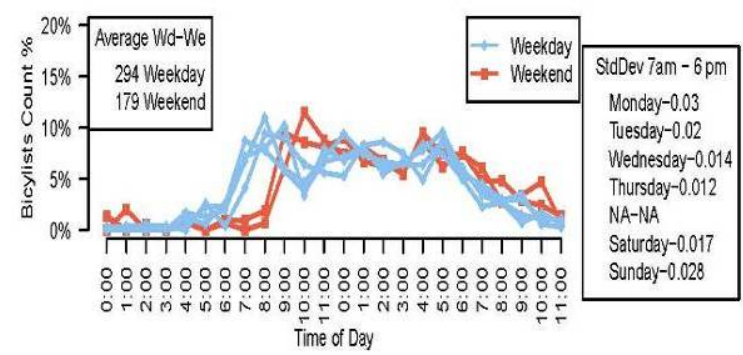

FrohnmayerSouthRiver

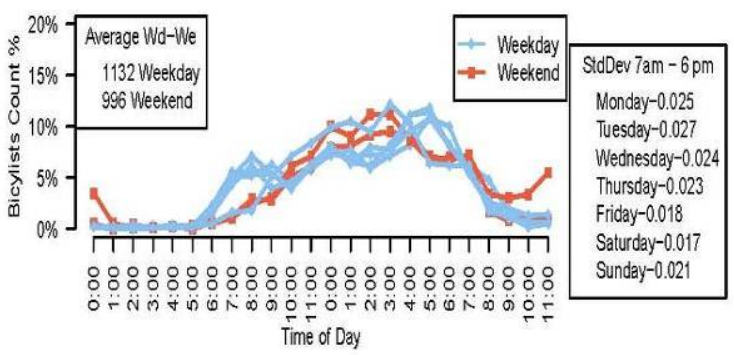

24thWestAdams

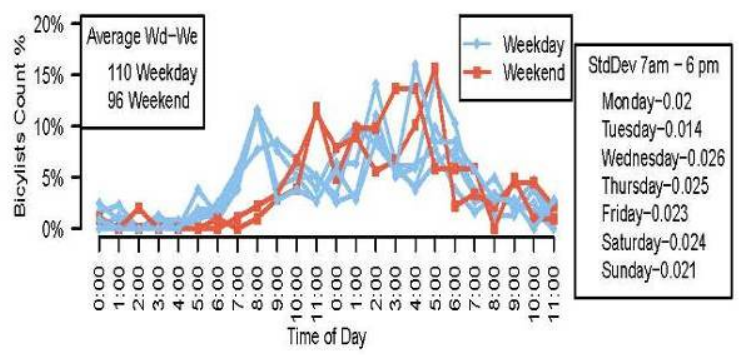

AgateSouthFranklin

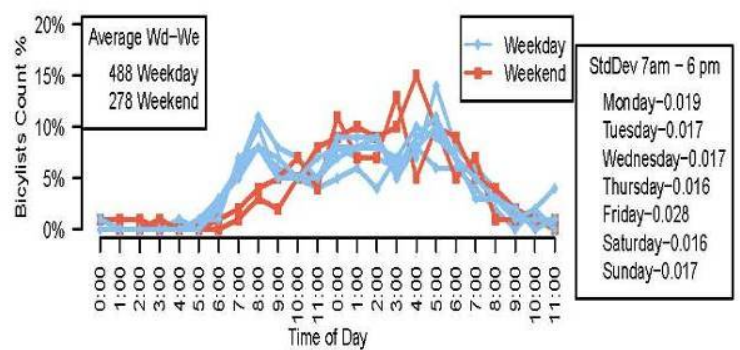

DefazioSouthRiverEastBridge

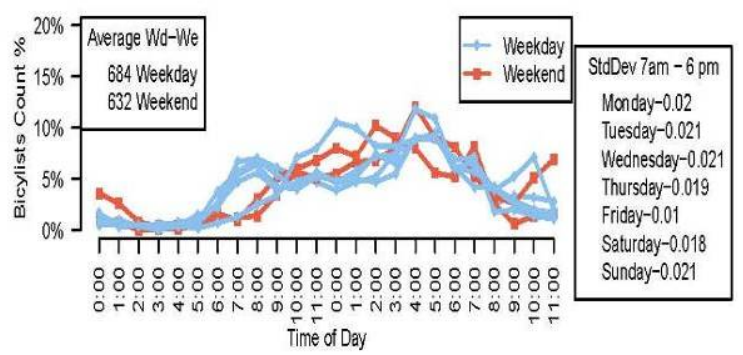

HarrisSouth18th

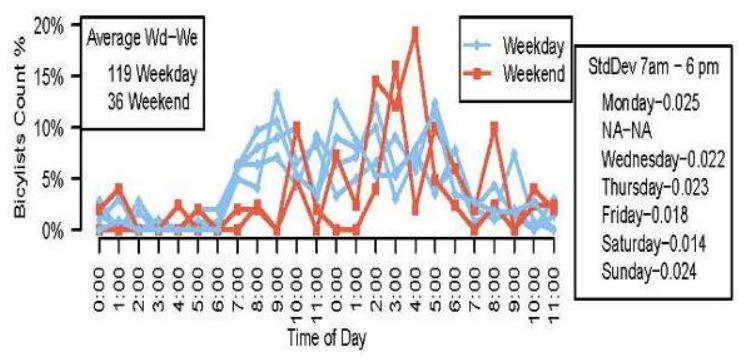




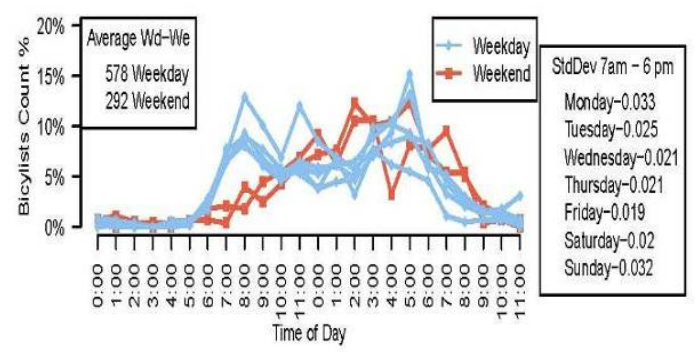

UniversitySouth18th

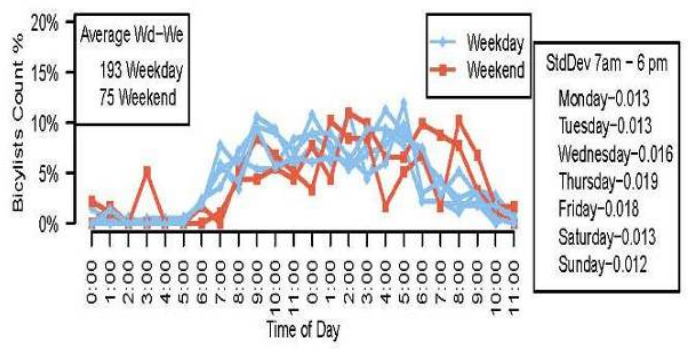

PolkNorth24th

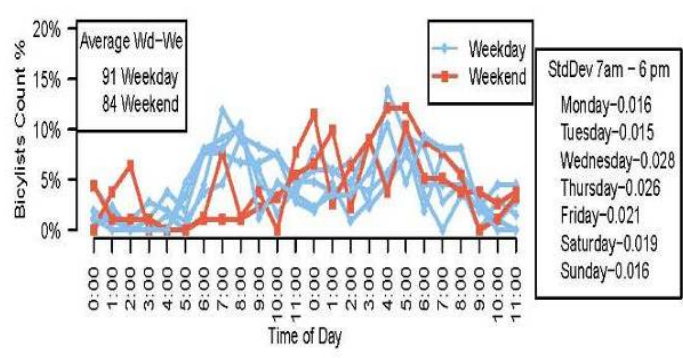

UniversitySouth24th

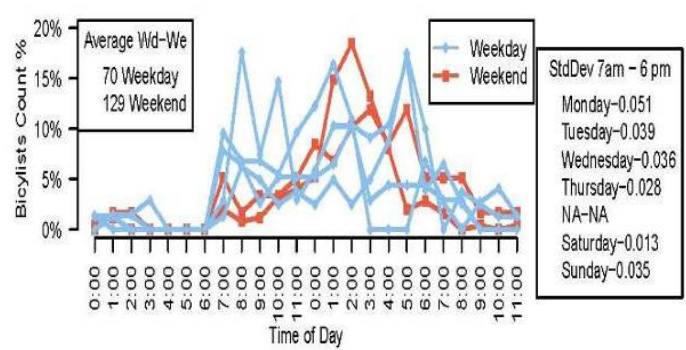


The lack of variation in hourly distributions as measured by the uniformity metric is what would be expected since locations serving primarily university users would probably experience steady traffic throughout the day as university students travel to and from campus as opposed to a standard commute pattern where the hourly distribution exhbits a strong morning and evening peak. To get a clearer idea of the university travel pattern compared to the traffic patterns discussed in Moreno-Miranda (2013), an example of each of the three traffic patterns has been constructed in Figure 3.5 using data gathered in the study area. 
Figure 3.5 - Examples of Bicycle Travel Patterns Using Study Area Data
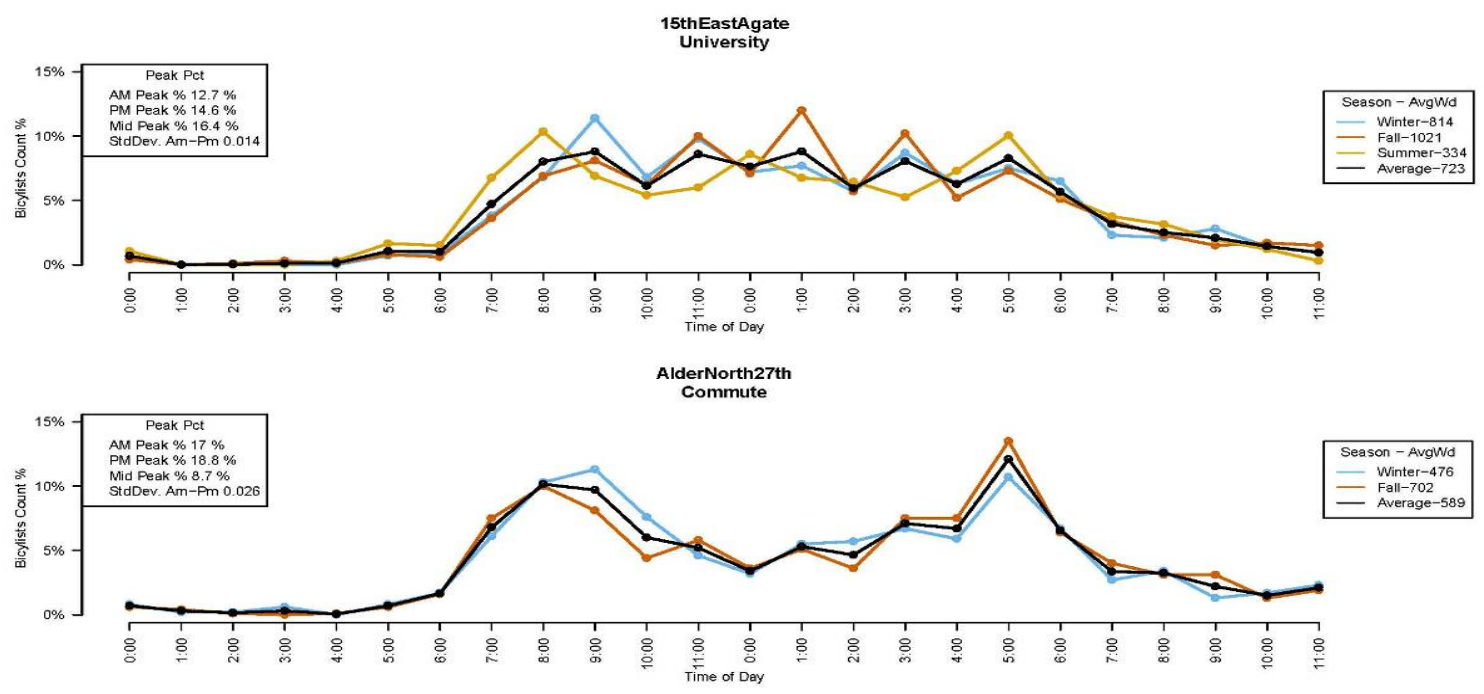

PioneerPkwySoutho
Recreation

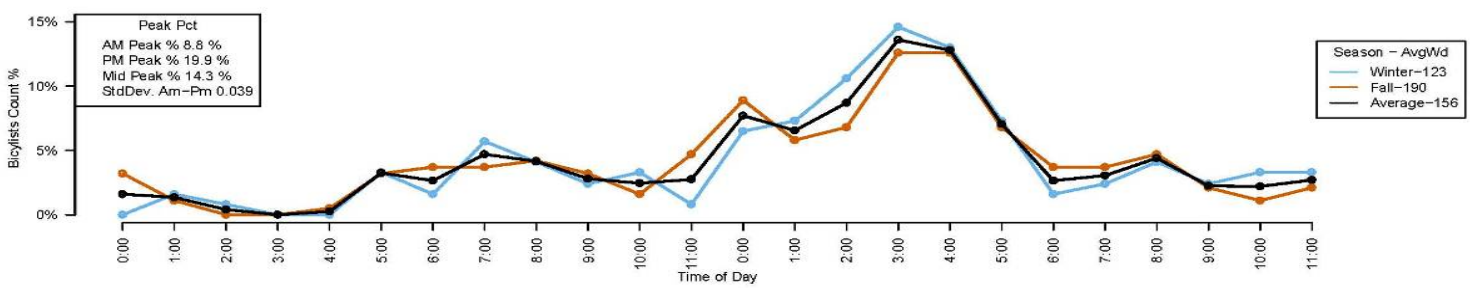

In Figure 3.5, the first plot demonstrates the hourly distribution of a typical university travel pattern while the next two plots display a commute and recreation pattern respectively. The university plot shows how travel remains steady throughout the day while the Commute plot has distinct morning and afternoon peaks. The third plot in Figure 3.5 shows the hourly distribution on a recreational traffic pattern with a distinct and protracted afternoon-evening peak.

Using just the hourly distributions to inform the travel pattern is useful but, MorenoMiranda (2013) also used the average weekday compared to an average weekend to 
justify a particular classification. Unfortunately the data set from the study area lacks consistently gathered weekend data so this research cannot use average daily to inform the classification process. Data was collected on weekends at some locations featured in Figure 3.3 (and Appendix B) and would indicate that a university travel pattern has less travel on average, during weekends compared with weekdays. This difference between average weekday and weekend travel would make sense for a university travel pattern since classes are nottypically held on weekends and therefore daily travel overall would be expected to be much lower. Additional data needs to be collected to more firmly establish characteristics of a "University" travel pattern. However, from current data collected from the study region, this research proposes two possible characteristics of a university travel pattern presented in Figure 3.6 below.

Figure 3.6 - Characteristic of University Travel Pattern

1. Generally flat hourly distribution between the morning and evening peak periods

2. Higher levels of traffic observed on weekdays compared to weekends

These characteristics do not result in peak factors that appear to be consistent enough to use as time-of-day extrapolation factors however. Table 3.7 below details the range of $A M$ and PM peak factors for locations exhibiting a university travel pattern and those that do not according to the two characteristics found in Figure 3.7 above. This finding 
would have implications if a university factor group were to be created and tested as was done using other types of patterns of usage as discussed in the next section.

\section{Table 3.7 - Range of AM and PM Peak Factors for Locations Exhibiting University Travel Patterns}

\begin{tabular}{|c|c|c|}
\hline $\begin{array}{c}\text { Exhibits University Travel } \\
\text { Pattern }\end{array}$ & $\begin{array}{c}\text { AM } \\
\text { Range }\end{array}$ & $\begin{array}{c}\text { PM } \\
\text { Range }\end{array}$ \\
\hline TRUE & $9-21 \%$ & $13-23 \%$ \\
\hline FALSE & $6-23 \%$ & $10-30 \%$ \\
\hline
\end{tabular}

\section{5 - Validating the Application of Time-of-Day Factors}

Factoring methods aim to improve data coverage in both time and geographic coverage. Factoring methods are also used to extrapolate counts from sites to similar locations, larger geographies or generalize data for modeling purposes. These methods usually use a baseline data set, a sample data set of field data and statistical methods to process data.

The metropolitan planning organization in the study region has been collecting data for over 8 months using automatic pneumatic tube counters capable of observing multiple full day bicycle travel at count stations around the study region. Data collected in the study region will be used to derive two sets (Scenario 1 and Scenario 2) of timeof-day expansion factors based on different factor grouping strategies. These will then be used to estimate 24-hour counts and compared against estimates derived from time- 
of-day expansion factors taken from the nationally recognized NBPDP bicycle data collection program. The application of each set of factors is a relatively simple calculation with descriptions of the computation below. All of the calculations are performed in the R open source statistical language which allows for quick and repeatable analysis. All $\mathrm{R}$ code is available as an appendix (Appendix D).

As discussed in the literature review, federal and state transportation agencies have developed guidance for motorized traffic extrapolation factoring but have yet to do so for non-motorized traffic such as bicycles. Short-term counts are many times the only data collected for bicycle traffic (FHWA 2005; FHWA 2011) and factoring allows for approximating more meaningful estimates (e.g. daily, weekly, monthly, and yearly volumes). This process occurs by first developing factors from multiple long-term counts of typically 24-hours or more. To calculate factors from long term counts, simply calculate the average percentage of traffic observed for each hour or factor period.

To extrapolate a short duration count of two-hours taken during either the morning or evening peaks, the product of the short-term count and the factor will approximate the 24-hour total. A simple equation in Figure 3.7 shows the factoring calculation

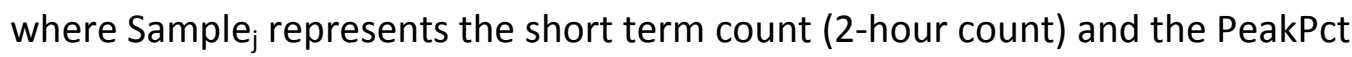
represents the assumed percentage of total daily volumes (DailyTraffic $\mathrm{c}_{\mathrm{i}}$ ).

Figure 3.7 - Time-of-Day Factoring

$$
\text { DailyTraffic } i_{i}=\left(\sum \text { Sample }_{j}\right) \div \text { PeakPct }
$$


Figure 3.8 uses example data from a single hourly distribution table to derive a short-term count (58 for the AM peak) and the factor (0.141) which when combined in the equation equal 411, which is nearly the observed 24-hour traffic count (minor discrepancy due to rounding). In order for this method to be reliable the peak period traffic proportion in the data where factors are constructed needs to be relatively close to the data in which the short-term count is being done. Reliability is accomplished by the creation of factor groups where count stations are classified by their traffic behavior as described in the section 3.3 above.

Figure 3.8 - Example of Application of Time-of-Day Factor

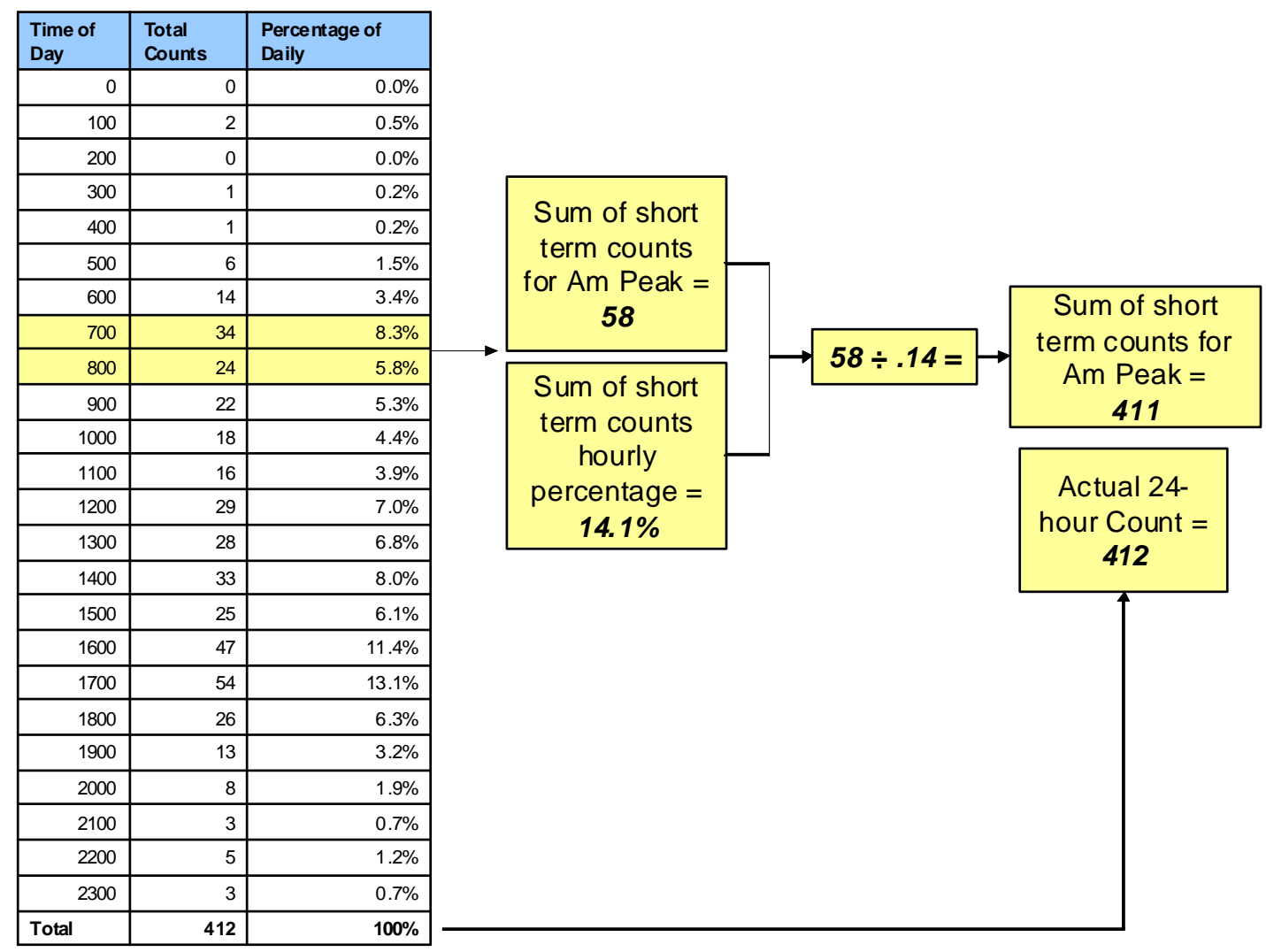




\subsection{1 - Time-of-Day Factors from Study Area Data - Scenario 1}

Data collected from 20 locations within the study area during the summer of 2012 were used to create four groups of time-of-day peak period factors. Factor groups were created by separating the data by bicycle facility type (Multi-use Regional Path, Bike Lane, or Bike Boulevard) or where no bicycle facility existed at all (No Facility). These time-of-day factors are summarized in Table 3.8 below and are the same factors presented in Table 3.5 above for Scenario 1.

Table 3.8 - Scenario 1 Time-of-Day Extrapolation Factors

\begin{tabular}{|c|c|c|c|}
\hline \multicolumn{3}{|c|}{ Time-of-Day Factor Comparison } \\
\hline Data set & $\begin{array}{c}\text { AM Peak Factor } \\
\text { (7-9 AM) }\end{array}$ & $\begin{array}{c}\text { PM Peak } \\
\text { Factor (4-6) }\end{array}$ \\
\hline & Average & 0.13 & 0.18 \\
\cline { 2 - 4 } & Path & 0.11 & 0.21 \\
\cline { 2 - 4 } & Lane & 0.13 & 0.16 \\
\cline { 2 - 4 } & Blvd & 0.13 & 0.18 \\
\cline { 2 - 4 } $\begin{array}{c}\text { Study Area Counts - } \\
\text { Scenario 1 }\end{array}$ & No Facility & 0.13 & 0.17 \\
\hline
\end{tabular}

In order to test the reliability of these time-of-day factors they will be applied to independent data collected in a separate season from the study region. Figures 3.9 and 3.10 describe the spatial location of each set of data. Tables 3.9 and 3.10 describe the total daily volumes for Tuesday and Thursday data collected at the factor creation and application sites. 
Table 3.9- Scenario 1 Factor Creation Location Daily Volumes

\begin{tabular}{|l|c|c|c|}
\hline Location & Thursday & Tuesday & $\begin{array}{c}\text { Location } \\
\text { Id }\end{array}$ \\
\hline DefazioSouthRiverEastBridge & 650 & 685 & 5 \\
\hline 11thWestAlder & 180 & 209 & 72 \\
\hline 12thEastLincoln & 513 & 492 & 28 \\
\hline 12thWestHigh & 372 & 344 & 12 \\
\hline 13thWestAlder & 1392 & 1335 & 21 \\
\hline 15thEastAgate & 340 & 327 & 24 \\
\hline 15thWestAlder & 469 & 477 & 26 \\
\hline 16thWestAlder & 167 & 140 & 70 \\
\hline 18thWestAlder & 352 & 349 & 46 \\
\hline 24thEastFilmore & 93 & 101 & 88 \\
\hline 24thWestAdams & 117 & 129 & 89 \\
\hline AgateSouth18th & 545 & 559 & 25 \\
\hline AgateSouthFranklin & 572 & 530 & 23 \\
\hline AlderSouth18th & 1227 & 1130 & 20 \\
\hline AlderSouthFranklin & 345 & 299 & 27 \\
\hline FrohnmayerSouthRiver & 1094 & 1084 & 6 \\
\hline HarrisSouth18th & 121 & $\mathrm{NA}$ & 73 \\
\hline OnyxNorthFranklin & 702 & 658 & 22 \\
\hline PolkNorth24th & 105 & 88 & 90 \\
\hline UniversitySouth18th & 203 & 213 & 71 \\
\hline
\end{tabular}


Table 3.10- Scenario 1 Factor Application Location Daily Volumes

\begin{tabular}{|l|c|c|c|}
\hline Location & Thursday & Tuesday & Location Id \\
\hline 15thWestJefferson & NA & 798 & 29 \\
\hline 32ndSouthOregon & 878 & NA & 36 \\
\hline AlderNorth27th & 702 & NA & 18 \\
\hline AmazonPathNorth24th & 1023 & NA & 8 \\
\hline DEastPioneerPkwyPath & 75 & NA & 38 \\
\hline DefazioSouthRiverEastBridge & 566 & NA & 5 \\
\hline DeltaBPBridgeEastGoodPasture & 207 & NA & 31 \\
\hline EastbankSouthOwossoBr & 488 & NA & 59 \\
\hline EWEBPathEast5th & NA & 76 & 34 \\
\hline EWEBPathEastPioneerPkwy & NA & 47 & 33 \\
\hline FernRidgeEastDanebo & NA & 178 & 9 \\
\hline FirEastRiverRd & 319 & NA & 19 \\
\hline FriendlySouth18th & NA & 217 & 14 \\
\hline GatewayBPBridgeEastl5 & 95 & NA & 40 \\
\hline GatewayStreetEastl5 & 42 & NA & 44 \\
\hline GoodpastureEastDeltaHwy & 149 & NA & 1 \\
\hline GWestMohawk & NA & 112 & 35 \\
\hline HarlowEastl5 & 55 & NA & 41 \\
\hline HeronBridgeSouthFernRidge & NA & 372 & 10 \\
\hline HighNorth13th & NA & 299 & 30 \\
\hline HighSouth4th & 718 & NA & 48 \\
\hline MonroeSouth8th & 478 & NA & 11 \\
\hline NorthbankEastKnickerbocker & 596 & NA & 97 \\
\hline NorthbankSouthGreenwayBr & NA & 509 & 3 \\
\hline NorthbankWestDeltaHwy & 338 & NA & 16 \\
\hline PearlSouth19th & 119 & NA & 17 \\
\hline PioneerPkwySouthQ & NA & 190 & 39 \\
\hline RichardsonBridge & NA & 427 & 50 \\
\hline SouthbankSouthGreenwayBr & NA & 1098 & 4 \\
\hline ThurstonEast58th & 44 & NA & 37 \\
\hline UniversityNorth24th & 220 & NA & 15 \\
\hline WillametteNorth15th & NA & 330 & 13 \\
\hline
\end{tabular}


Figure 3.9 - Scenario 1 Time-of-Day Factor Creation Locations (20 locations)

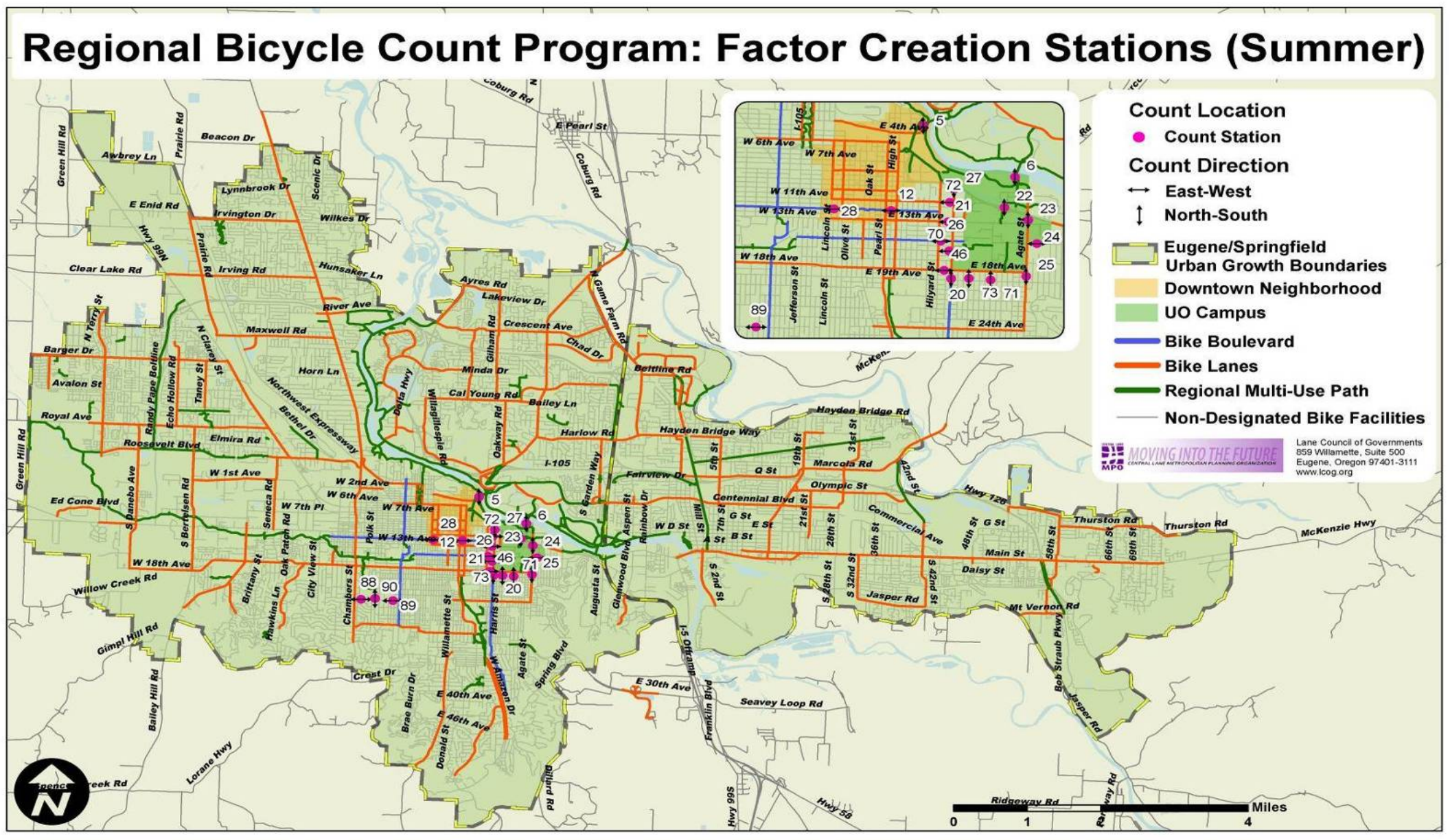


Figure 3.10 - Scenario 1 Time-of-Day Factor Application Locations (32 locations)

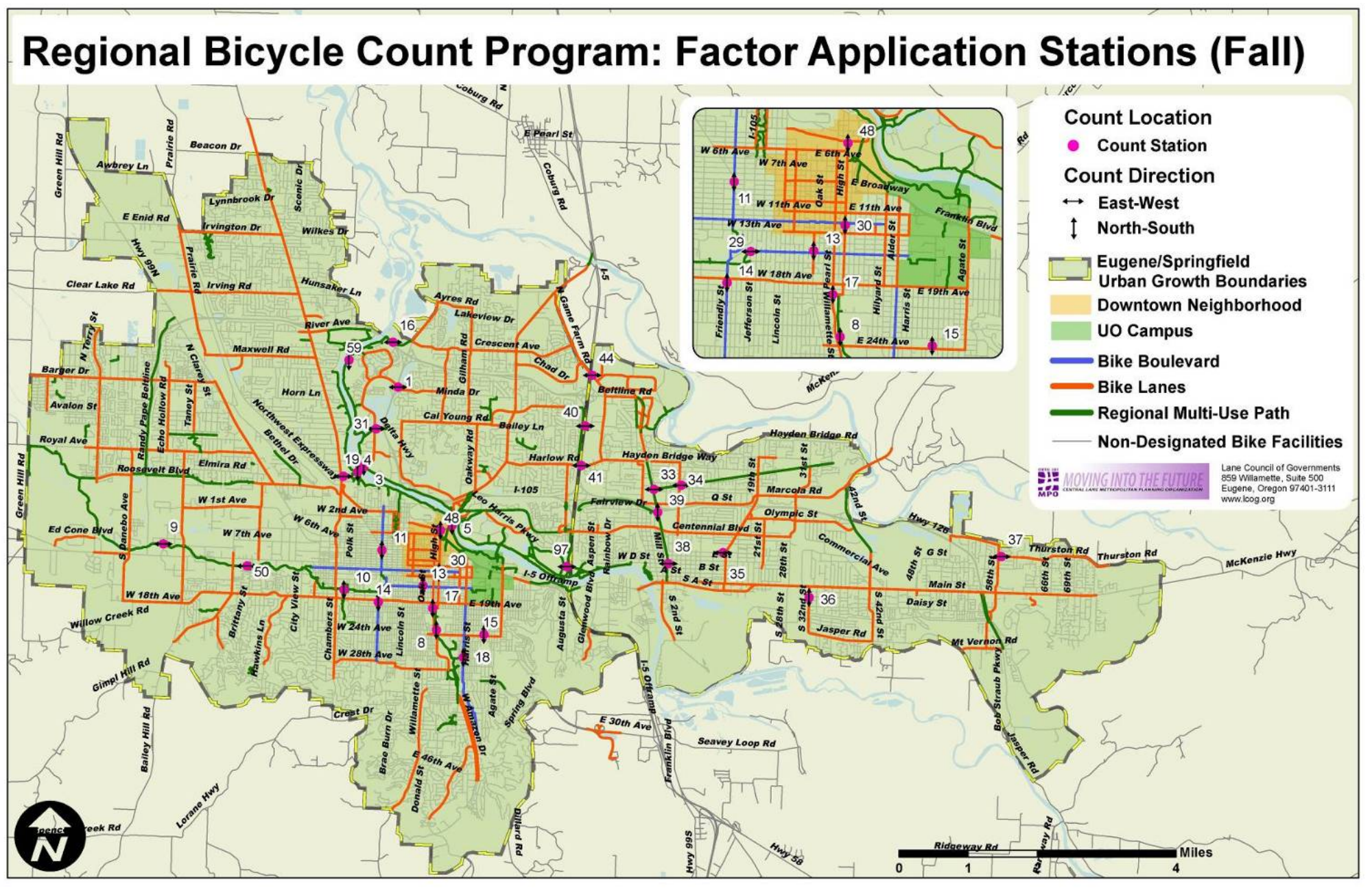


Factors from study area data were put into factor groups based on the bicycle facility type(or lack of bike facility) in order to increase the reliability of the estimation process and provide for sensible groupings of factors. Since the data used to create the factors are independent(i.e. different locations) of the data in which the factors are applied this validation will test how well these factor groupings work when appllied simulate how some agencies might actually create and apply time-of-day factors. It is important to point out again that all data are representing travel on either a Tuesday or a Thursday since the structure of the study area's bike count program collects information only on these days.

The factors used from the National Bicycle and Pedestrian Documentation Project (NBPDP) are not broken into detailed factor groups. These factors have been taken from the NBPDP methodology and spreadsheet tool used to estimate daily traffic from short-term counts and the NBPDP methodology only provides two facility types, pedestrian district and off-street path. For the purposes of reasonable comparison with factors derived from study area data, the off-street path factors will be used since that facility type most closely resembles the types of facilities where the study area data were collected. Time of year is considered as well, as the NBPDP factors are broken into two groups, one for April to September and the other for October to March. The application of factors in Scenario 1 will apply time-of-day factors to data collected during the fall, during the months of October and November, so the latter time period 
will be used for the NPBPD factors since it adheres to a similar time period when study area data was collected.

The estimation process using NBPDP factors is similar to the method using study area derived factors described above but uses different time-of-day factors and also has a final step to account for travel not observed in the hourly percentages. According to the NBPDP documentation, the factoring calculation only accounts for the hours between 6:00 AM and 10:00 PM, the time period in which 95\% of all bicycle travel occurs. An additional step then accounts for the remaining $5 \%$ by using a 1.05 multiplier at the end of the calculation. The equation for the NBPDP calculation is described in Figure 3.11 below, where Sample is $_{\mathrm{j}}$ the sample taken at location $\mathrm{i}$ and PeakPct is the average peak percentage assumed by the NBPDP.

\section{Figure 3.11 - NBPDP Time-of-Day Factoring$$
\text { DailyTraffic } \left._{i}=\left(\sum \text { Sample }_{j}\right) \div \text { PeakPct }\right) \times 1.05
$$

The premise of testing the time-of-day factors comes from the current state of the practice for bicycle counts summarized in two Federal Highways Administration reports (FHWA 2007; FHWA 2011) discussed above, that found most count programs consisting of manual 2-hour bicycle traffic counts. This research simulates a possible scenario where 24-hour data is collected for a number of locations where time-of-day factors are derived and then applied to simulated two-hour sample counts. These two-hour sample 
counts are "simulated" because in reality 24-hours of counts exist, which allows for comparison with the estimated 24-hour count to verify the estimation results. The process of applying the study area factors and NBPDP factors is summarized below in Figure 3.12 .

\section{Figure 3.12 - Summary of Application of Time-of-Day Factors for Scenario 1}

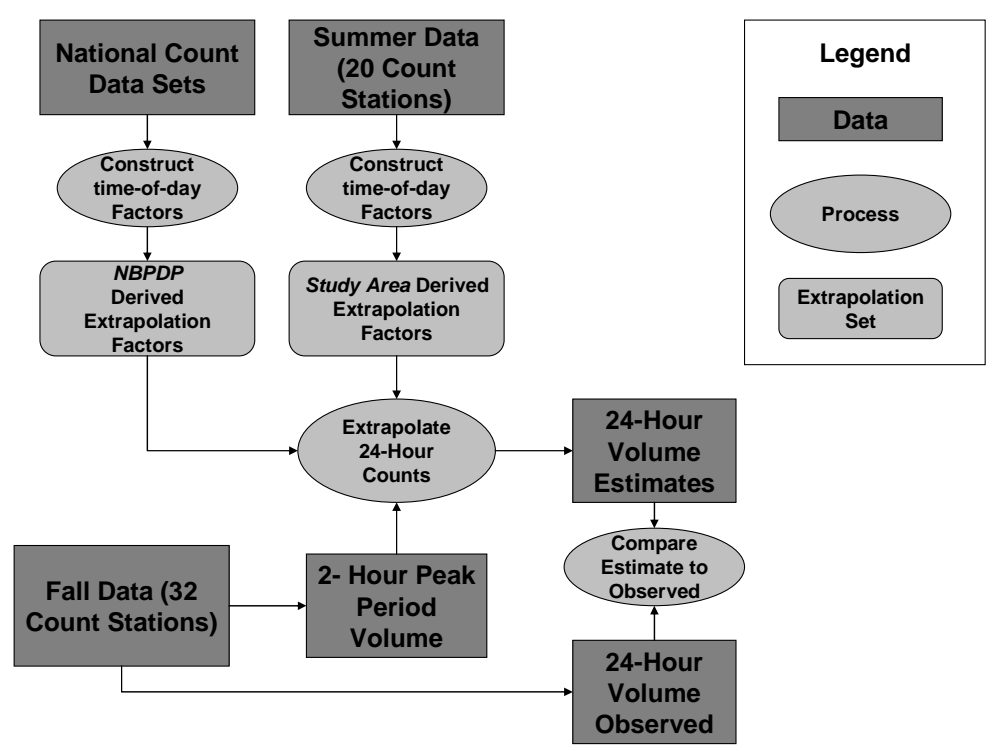

Using data collected from 20 count sites in the summer of 2012, time-of-day factors have been constructed and applied to sample peak period (both morning and evening) two-hour volumes pulled from observed 24-hour counts done in fall of 2012 collected from independent count stations. The same application process was completed using 
factors derived from the NBPDP. The results from each application of factors are presented in the tables and figures below.

Tables 3.11 and 3.12 detail the observed and estimated results using study area derived time-of-day factors using morning and evening factors and 2-hour samples. The results table also includes the percent difference and the absolute percent difference.

Figure 3.13 describe how these measures of error have been calculated.

\section{Figure 3.13 - Calculation of Measure of Error}

$$
\begin{aligned}
& \text { Error as \% Difference }=\left(\mathrm{DBT}_{\mathrm{e}}-\mathrm{DBT}\right) / \mathrm{DBT} \\
& \text { Error as Absolute \% Difference }=\mid\left(\mathrm{DBT} T_{\mathrm{e}}-\mathrm{DBT} \mid / \mathrm{DBT}\right. \\
& \text { where } \\
& \mathrm{DBT}_{\mathrm{e}}=\text { estimated Daily Bicycle Traffic } \\
& \mathrm{DBT}=\text { actual Daily Bicycle Traffic }
\end{aligned}
$$

Results from the application of the NBPDP factors are then presented in Tables 3.13 and 3.14 with the same measures of error included. 
Table 3.11 - AMPeak Factor Results using Study Area Factors

\begin{tabular}{|c|c|c|c|c|c|c|c|}
\hline \multicolumn{8}{|c|}{ Study Area - AM Factor } \\
\hline Location & Observed & Estimate & $\begin{array}{l}\% \\
\text { Difference }\end{array}$ & $\begin{array}{l}\text { Absolute } \\
\% \\
\text { Difference } \\
\end{array}$ & $\begin{array}{l}\text { Factor } \\
\text { Group }\end{array}$ & $\begin{array}{l}\text { Federal } \\
\text { Functional } \\
\text { Class }\end{array}$ & $\begin{array}{l}\text { Location } \\
\text { Id }\end{array}$ \\
\hline GoodpastureEastDeltaHwy & 149 & 148 & $99 \%$ & $1 \%$ & Lane & $\begin{array}{l}\text { Major } \\
\text { Collector }\end{array}$ & 1 \\
\hline UniversityNorth24th & 220 & 215 & $98 \%$ & $2 \%$ & NoFacility & Local & 15 \\
\hline DefazioSouthRiverEastBridge & 566 & 591 & $104 \%$ & $4 \%$ & Path & Path & 5 \\
\hline GWestMohawk & 112 & 108 & $96 \%$ & $4 \%$ & NoFacility & $\begin{array}{l}\text { Major } \\
\text { Collector }\end{array}$ & 35 \\
\hline HighSouth4th & 718 & 762 & $106 \%$ & $6 \%$ & NoFacility & $\begin{array}{l}\text { Major } \\
\text { Collector }\end{array}$ & 48 \\
\hline NorthbankEastKnickerbocker & 596 & 555 & $93 \%$ & $7 \%$ & Path & Path & 97 \\
\hline GatewayStreetEastl5 & 42 & 39 & $93 \%$ & $7 \%$ & Lane & Minor Arterial & 44 \\
\hline SouthbankSouthGreenwayBr & 1098 & 1009 & $92 \%$ & $8 \%$ & Path & Path & 4 \\
\hline DEastPioneerPkwyPath & 75 & 69 & $92 \%$ & $8 \%$ & NoFacility & Local & 38 \\
\hline 15thWestJefferson & 798 & 867 & $109 \%$ & $9 \%$ & Blvd & Local & 29 \\
\hline DeltaBPBridgeEastGoodPasture & 207 & 227 & $110 \%$ & $10 \%$ & Path & Path & 31 \\
\hline HarlowEastl5 & 55 & 62 & $113 \%$ & $13 \%$ & Lane & Minor Arterial & 41 \\
\hline NorthbankSouthGreenwayBr & 509 & 418 & $82 \%$ & $18 \%$ & Path & Path & 3 \\
\hline MonroeSouth8th & 478 & 375 & $78 \%$ & $22 \%$ & Blvd & $\begin{array}{l}\text { Major } \\
\text { Collector }\end{array}$ & 11 \\
\hline FriendlySouth18th & 217 & 266 & $123 \%$ & $23 \%$ & Blvd & $\begin{array}{l}\text { Major } \\
\text { Collector }\end{array}$ & 14 \\
\hline PioneerPkwySouthQ & 190 & 136 & $72 \%$ & $28 \%$ & Path & Path & 39 \\
\hline PearlSouth19th & 119 & 86 & $72 \%$ & $28 \%$ & Lane & $\begin{array}{l}\text { Major } \\
\text { Collector }\end{array}$ & 17 \\
\hline FirEastRiverRd & 319 & 231 & $72 \%$ & $28 \%$ & NoFacility & Local & 19 \\
\hline EastbankSouthOwossoBr & 488 & 336 & $69 \%$ & $31 \%$ & Path & Path & 2 \\
\hline FernRidgeEastDanebo & 178 & 236 & $133 \%$ & $33 \%$ & Path & Path & 9 \\
\hline RichardsonBridge & 427 & 582 & $136 \%$ & $36 \%$ & Path & Path & 32 \\
\hline
\end{tabular}




\begin{tabular}{|l|r|r|r|r|l|l|l|} 
AlderNorth27th & 702 & 961 & $137 \%$ & $37 \%$ & Blvd & Local \\
\hline WillametteNorth15th & 330 & 203 & $62 \%$ & $38 \%$ & Lane & Minor Arterial & 13 \\
\hline HeronBridgeSouthFernRidge & 372 & 518 & $139 \%$ & $39 \%$ & Path & Path \\
\hline NorthbankWestDeltaHwy & 338 & 191 & $57 \%$ & $43 \%$ & Path & Path & 10 \\
\hline 32ndSouthOregon & 878 & 445 & $51 \%$ & $49 \%$ & Lane & Minor Arterial & 36 \\
\hline AmazonPathNorth24th & 1023 & 1782 & $174 \%$ & $74 \%$ & Path & Path \\
\hline EWEBPathEastPioneerPkwy & 47 & 82 & $174 \%$ & $74 \%$ & Path & Path & Minor Arterial \\
\hline HighNorth13th & 299 & 523 & $175 \%$ & $75 \%$ & Lane & 33 \\
\hline EWEBPathEast5th & 76 & 136 & $179 \%$ & $79 \%$ & Path & Path & 34 \\
\hline GatewayBPBridgeEastl5 & 95 & 200 & $211 \%$ & $111 \%$ & Path & Path & 34 \\
\hline ThurstonEast58th & 44 & 117 & $266 \%$ & $166 \%$ & Lane & Minor Arterial & 37 \\
\hline
\end{tabular}


Table 3.12 - PM Peak Factor Results using Study Area Factors

\begin{tabular}{|c|c|c|c|c|c|c|c|}
\hline \multicolumn{8}{|c|}{ Study Area - PM Factor } \\
\hline Location & Observed & Estimate & $\begin{array}{l}\% \\
\text { Difference }\end{array}$ & $\begin{array}{l}\text { Absolute } \\
\% \\
\text { Difference }\end{array}$ & $\begin{array}{l}\text { Factor } \\
\text { Group }\end{array}$ & $\begin{array}{l}\text { Federal } \\
\text { Functional } \\
\text { Class }\end{array}$ & $\begin{array}{l}\text { Location } \\
\text { Id }\end{array}$ \\
\hline MonroeSouth8th & 478 & 484 & $101 \%$ & $1 \%$ & Blvd & $\begin{array}{l}\text { Major } \\
\text { Collector }\end{array}$ & 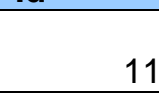 \\
\hline HighSouth4th & 718 & 713 & $99 \%$ & $1 \%$ & NoFacility & $\begin{array}{l}\text { Major } \\
\text { Collector }\end{array}$ & 48 \\
\hline AmazonPathNorth24th & 1023 & 1043 & $102 \%$ & $2 \%$ & Path & Path & 8 \\
\hline GoodpastureEastDeltaHwy & 149 & 154 & $103 \%$ & $3 \%$ & Lane & $<N A>$ & 1 \\
\hline WillametteNorth15th & 330 & 321 & $97 \%$ & $3 \%$ & Lane & Minor Arterial & 13 \\
\hline DefazioSouthRiverEastBridge & 566 & 595 & $105 \%$ & $5 \%$ & Path & Path & 5 \\
\hline PioneerPkwySouthQ & 190 & 176 & $93 \%$ & $7 \%$ & Path & Path & 39 \\
\hline HighNorth13th & 299 & 278 & $93 \%$ & $7 \%$ & Lane & Minor Arterial & 30 \\
\hline HeronBridgeSouthFernRidge & 372 & 405 & $109 \%$ & $9 \%$ & Path & Path & 10 \\
\hline PearlSouth19th & 119 & 130 & $109 \%$ & $9 \%$ & Lane & $\begin{array}{l}\text { Major } \\
\text { Collector }\end{array}$ & 17 \\
\hline NorthbankEastKnickerbocker & 596 & 662 & $111 \%$ & $11 \%$ & Path & Path & 97 \\
\hline NorthbankWestDeltaHwy & 338 & 376 & $111 \%$ & $11 \%$ & Path & Path & 16 \\
\hline HarlowEastl5 & 55 & 49 & $89 \%$ & $11 \%$ & Lane & Minor Arterial & 41 \\
\hline EWEBPathEast5th & 76 & 67 & $88 \%$ & $12 \%$ & Path & Path & 34 \\
\hline FirEastRiverRd & 319 & 356 & $112 \%$ & $12 \%$ & NoFacility & Local & 19 \\
\hline RichardsonBridge & 427 & 481 & $113 \%$ & $13 \%$ & Path & Path & 32 \\
\hline FernRidgeEastDanebo & 178 & 205 & $115 \%$ & $15 \%$ & Path & Path & 9 \\
\hline GWestMohawk & 112 & 95 & $85 \%$ & $15 \%$ & NoFacility & $\begin{array}{l}\text { Major } \\
\text { Collector }\end{array}$ & 35 \\
\hline AlderNorth27th & 702 & 813 & $116 \%$ & $16 \%$ & Blvd & Local & 18 \\
\hline EWEBPathEastPioneerPkwy & 47 & 57 & $121 \%$ & $21 \%$ & Path & Path & 33 \\
\hline SouthbankSouthGreenwayBr & 1098 & 1348 & $123 \%$ & $23 \%$ & Path & Path & 4 \\
\hline UniversityNorth24th & 220 & 273 & $124 \%$ & $24 \%$ & NoFacility & Local & 15 \\
\hline
\end{tabular}




\begin{tabular}{|l|r|r|r|r|l|l|r|} 
15thWestJefferson & 798 & 1022 & $128 \%$ & $28 \%$ & Blvd & Local & \\
\hline FriendlySouth18th & 217 & 280 & $129 \%$ & $29 \%$ & Blvd & $\begin{array}{l}\text { Major } \\
\text { Collector }\end{array}$ & 14 \\
\hline ThurstonEast58th & 44 & 31 & $70 \%$ & $30 \%$ & Lane & Minor Arterial & 37 \\
\hline GatewayBPBridgeEast15 & 95 & 129 & $136 \%$ & $36 \%$ & Path & Path & 40 \\
\hline EastbankSouthOwossoBr & 488 & 676 & $139 \%$ & $39 \%$ & Path & Path & 2 \\
\hline NorthbankSouthGreenwayBr & 509 & 729 & $143 \%$ & $43 \%$ & Path & Path & 3 \\
\hline DEastPioneerPkwyPath & 75 & 113 & $151 \%$ & $51 \%$ & NoFacility & Local & 38 \\
\hline 32ndSouthOregon & 878 & 1333 & $152 \%$ & $52 \%$ & Lane & Minor Arterial & 36 \\
\hline DeltaBPBridgeEastGoodPasture & 207 & 329 & $159 \%$ & $59 \%$ & Path & Path & 31 \\
\hline GatewayStreetEastl5 & 42 & 12 & 0.29 & $71 \%$ & Lane & Minor Arterial & 44 \\
\hline
\end{tabular}


Table 3.13 - AM Peak Factor Results using NBPDP Factors

\begin{tabular}{|c|c|c|c|c|c|c|c|}
\hline \multicolumn{5}{|c|}{ NBPDP - AM Factor } & \multirow{2}{*}{$\begin{array}{l}\text { Factor } \\
\text { Group }\end{array}$} & \multirow{2}{*}{$\begin{array}{l}\text { Functional } \\
\text { Class }\end{array}$} & \multirow{2}{*}{$\begin{array}{l}\text { Location } \\
\text { Id }\end{array}$} \\
\hline Location & Observed & Estimate & $\begin{array}{l}\% \\
\text { Difference }\end{array}$ & $\begin{array}{l}\text { Absolute } \\
\% \\
\text { Difference }\end{array}$ & & & \\
\hline FirEastRiverRd & 319 & 315 & $99 \%$ & $1 \%$ & NoFacility & Local & 19 \\
\hline PearlSouth19th & 119 & 116 & $97 \%$ & $3 \%$ & Lane & $\begin{array}{l}\text { Major } \\
\text { Collector }\end{array}$ & 17 \\
\hline NorthbankSouthGreenwayBr & 509 & 483 & $95 \%$ & $5 \%$ & Path & Path & 3 \\
\hline MonroeSouth8th & 478 & 504 & $105 \%$ & $5 \%$ & Blvd & $\begin{array}{l}\text { Major } \\
\text { Collector }\end{array}$ & 11 \\
\hline SouthbankSouthGreenwayBr & 1098 & 1166 & $106 \%$ & $6 \%$ & Path & Path & 4 \\
\hline NorthbankEastKnickerbocker & 596 & 640 & $107 \%$ & $7 \%$ & Path & Path & 97 \\
\hline PioneerPkwySouthQ & 190 & 158 & $83 \%$ & $17 \%$ & Path & Path & 39 \\
\hline WillametteNorth15th & 330 & 273 & $83 \%$ & $17 \%$ & Lane & Minor Arterial & 13 \\
\hline DefazioSouthRiverEastBridge & 566 & 682 & $120 \%$ & $20 \%$ & Path & Path & 5 \\
\hline EastbankSouthOwossoBr & 488 & 388 & $80 \%$ & $20 \%$ & Path & Path & 2 \\
\hline GatewayStreetEastl5 & 42 & 52 & $124 \%$ & $24 \%$ & Lane & Minor Arterial & 44 \\
\hline DEastPioneerPkwyPath & 75 & 94 & $125 \%$ & $25 \%$ & NoFacility & Local & 38 \\
\hline DeltaBPBridgeEastGoodPasture & 207 & 262 & $127 \%$ & $27 \%$ & Path & Path & 31 \\
\hline GWestMohawk & 112 & 147 & $131 \%$ & $31 \%$ & NoFacility & $\begin{array}{l}\text { Major } \\
\text { Collector }\end{array}$ & 35 \\
\hline 32ndSouthOregon & 878 & 598 & $68 \%$ & $32 \%$ & Lane & Minor Arterial & 36 \\
\hline GoodpastureEastDeltaHwy & 149 & 200 & $134 \%$ & $34 \%$ & Lane & $<\mathrm{NA}>$ & 1 \\
\hline UniversityNorth24th & 220 & 294 & $134 \%$ & $34 \%$ & NoFacility & Local & 15 \\
\hline NorthbankWestDeltaHwy & 338 & 220 & $65 \%$ & $35 \%$ & Path & Path & 16 \\
\hline HighSouth4th & 718 & 1040 & $145 \%$ & $45 \%$ & NoFacility & $\begin{array}{l}\text { Major } \\
\text { Collector }\end{array}$ & 48 \\
\hline 15thWestJefferson & 798 & 1166 & $146 \%$ & $46 \%$ & Blvd & Loeal & 29 \\
\hline FernRidgeEastDanebo & 178 & 273 & $153 \%$ & $53 \%$ & Path & Path & 9 \\
\hline HarlowEastl5 & 55 & 84 & $153 \%$ & $53 \%$ & Lane & Minor Arterial & 41 \\
\hline
\end{tabular}




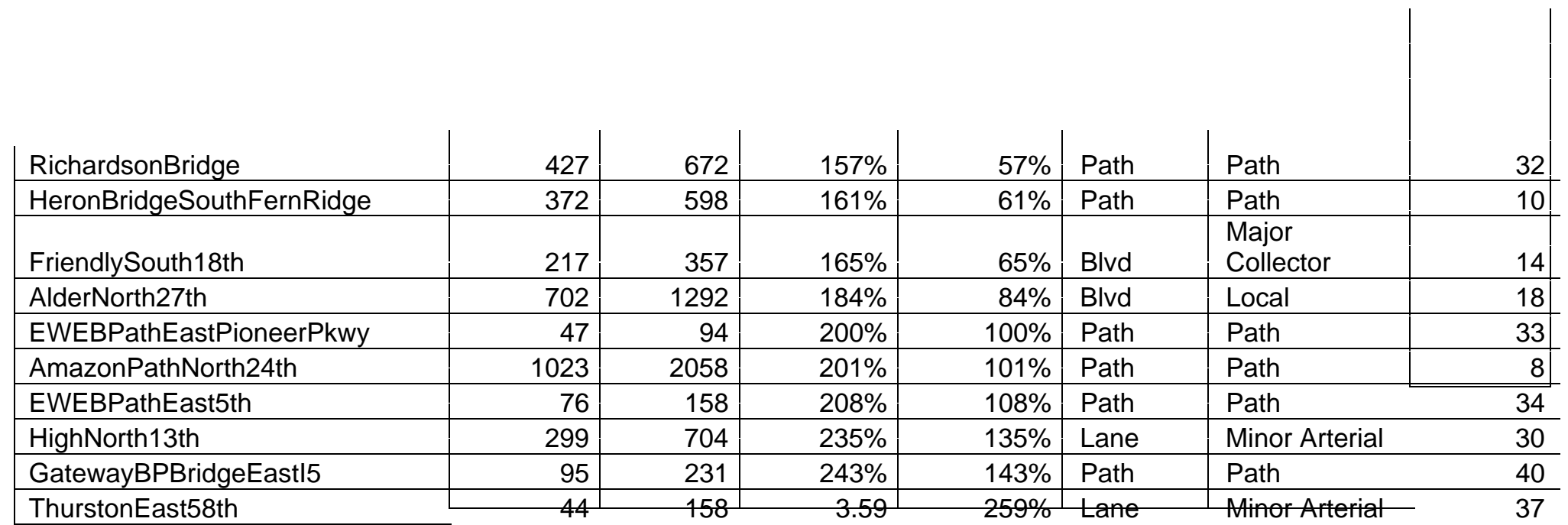


Table 3.14 - PM Peak Factor Results using NBPD Factors

\begin{tabular}{|c|c|c|c|c|c|c|c|}
\hline \multirow[b]{2}{*}{ Location } & \multicolumn{5}{|c|}{ NBPDP - PM Factor } & \multirow{2}{*}{$\begin{array}{l}\text { reueral } \\
\text { Functional } \\
\text { Class }\end{array}$} & \multirow{2}{*}{$\begin{array}{l}\text { Location } \\
\text { Id }\end{array}$} \\
\hline & Observed & Estimate & $\begin{array}{l}\% \\
\text { Difference }\end{array}$ & $\begin{array}{l}\text { Absolute \% } \\
\text { Difference }\end{array}$ & $\begin{array}{l}\text { Factor } \\
\text { Group }\end{array}$ & & \\
\hline GWestMohawk & 112 & 112 & $100 \%$ & $0 \%$ & NoFacility & $\begin{array}{l}\text { Major } \\
\text { Collector }\end{array}$ & 35 \\
\hline HarlowEastl5 & 55 & 56 & $102 \%$ & $2 \%$ & Lane & Minor Arterial & 41 \\
\hline HighNorth13th & 299 & 315 & $105 \%$ & $5 \%$ & Lane & Minor Arterial & 30 \\
\hline WillametteNorth15th & 330 & 364 & $110 \%$ & $10 \%$ & Lane & Minor Arterial & 13 \\
\hline GoodpastureEastDeltaHwy & 149 & 175 & $117 \%$ & $17 \%$ & Lane & $<\mathrm{NA}>$ & 1 \\
\hline HighSouth4th & 718 & 840 & $117 \%$ & $17 \%$ & NoFacility & $\begin{array}{l}\text { Major } \\
\text { Collector }\end{array}$ & 48 \\
\hline ThurstonEast58th & 44 & 35 & $80 \%$ & $20 \%$ & Lane & Minor Arterial & 37 \\
\hline PearlSouth19th & 119 & 147 & $124 \%$ & $24 \%$ & Lane & $\begin{array}{l}\text { Major } \\
\text { Collector }\end{array}$ & 17 \\
\hline EWEBPathEast5th & 76 & 98 & $129 \%$ & $29 \%$ & Path & Path & 34 \\
\hline MonroeSouth8th & 478 & 616 & $129 \%$ & $29 \%$ & Blvd & $\begin{array}{l}\text { Major } \\
\text { Collector }\end{array}$ & 11 \\
\hline FirEastRiverRd & 319 & 420 & $132 \%$ & $32 \%$ & NoFacility & Local & 19 \\
\hline PioneerPkwySouthQ & 190 & 259 & $136 \%$ & $36 \%$ & Path & Path & 39 \\
\hline UniversityNorth24th & 220 & 322 & $146 \%$ & $46 \%$ & NoFacility & Local & 15 \\
\hline AlderNorth27th & 702 & 1036 & $148 \%$ & $48 \%$ & Blvd & Local & 18 \\
\hline AmazonPathNorth24th & 1023 & 1533 & $150 \%$ & $50 \%$ & Path & Path & 8 \\
\hline DefazioSouthRiverEastBridge & 566 & 875 & $155 \%$ & $55 \%$ & Path & Path & 5 \\
\hline HeronBridgeSouthFernRidge & 372 & 595 & $160 \%$ & $60 \%$ & Path & Path & 10 \\
\hline NorthbankEastKnickerbocker & 596 & 973 & $163 \%$ & $63 \%$ & Path & Path & 97 \\
\hline 15thWestJefferson & 798 & 1302 & $163 \%$ & $63 \%$ & Blvd & Local & 29 \\
\hline NorthbankWestDeltaHwy & 338 & 553 & $164 \%$ & $64 \%$ & Path & Path & 16 \\
\hline FriendlySouth18th & 217 & 357 & $165 \%$ & $65 \%$ & Blvd & $\begin{array}{l}\text { Major } \\
\text { Collector }\end{array}$ & 14 \\
\hline
\end{tabular}




\begin{tabular}{|c|c|c|c|c|c|c|c|}
\hline RichardsonBridge & 427 & 707 & $166 \%$ & $66 \%$ & Path & Path & 32 \\
\hline GatewayStreetEastl5 & 42 & 14 & $33 \%$ & $67 \%$ & Lane & Minor Arterial & 44 \\
\hline FernRidgeEastDanebo & 178 & 301 & $169 \%$ & $69 \%$ & Path & Path & 9 \\
\hline 32ndSouthOregon & 878 & 1512 & $172 \%$ & $72 \%$ & Lane & Minor Arterial & 36 \\
\hline DEastPioneerPkwyPath & 75 & 133 & $177 \%$ & $77 \%$ & NoFacility & Local & 38 \\
\hline EWEBPathEastPioneerPkwy & 47 & 84 & $179 \%$ & $79 \%$ & Path & Path & 33 \\
\hline SouthbankSouthGreenwayBr & 1098 & 1981 & $180 \%$ & $80 \%$ & Path & Path & 4 \\
\hline GatewayBPBridgeEastl5 & 95 & 189 & $199 \%$ & $99 \%$ & Path & Path & 40 \\
\hline EastbankSouthOwossoBr & 488 & 994 & $204 \%$ & $104 \%$ & Path & Path & 2 \\
\hline NorthbankSouthGreenwayBr & 509 & 1071 & $210 \%$ & $110 \%$ & Path & Path & 3 \\
\hline DeltaBPBridgeEastGoodPasture & 207 & 483 & $233 \%$ & $133 \%$ & Path & Path & 31 \\
\hline
\end{tabular}


Figure 3.14 below plots each the results in Scenario 1 using both peak periods for each time-of-day factor set for a visual representation which includes some summary statistics for the overall performance for the estimation. Results using factors generated from study area data are featured in the top two plots with the NBPDP results in the bottom half of the figure.

Figure 3.14- Results for Scenario 1 Study Area Factors Compared to NBPDP Factors

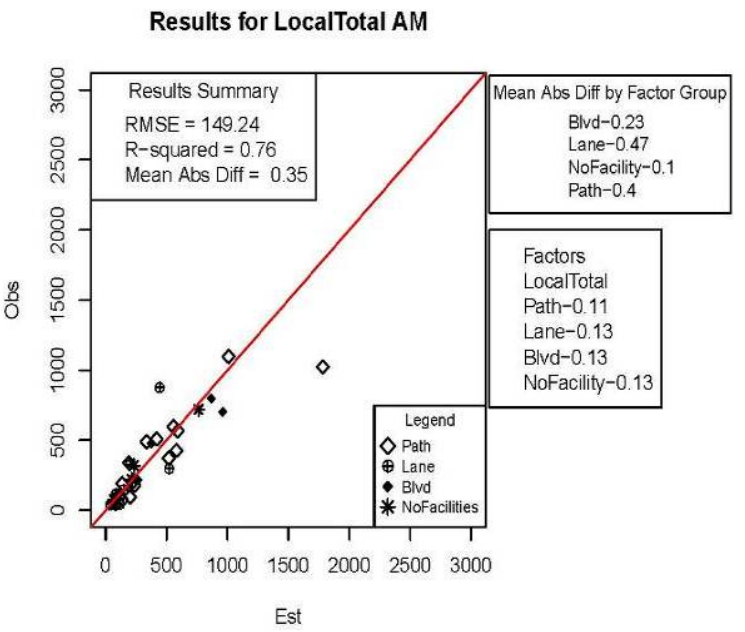

Results for NBPD AM

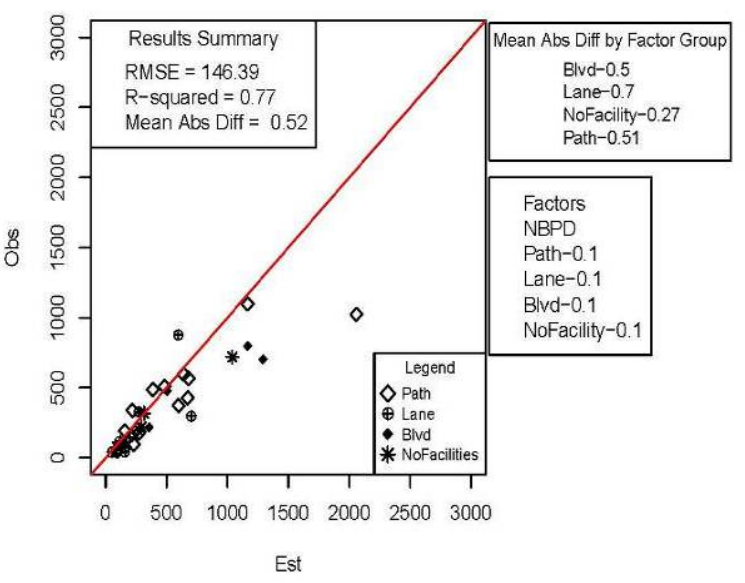

Results for LocalTotal PM

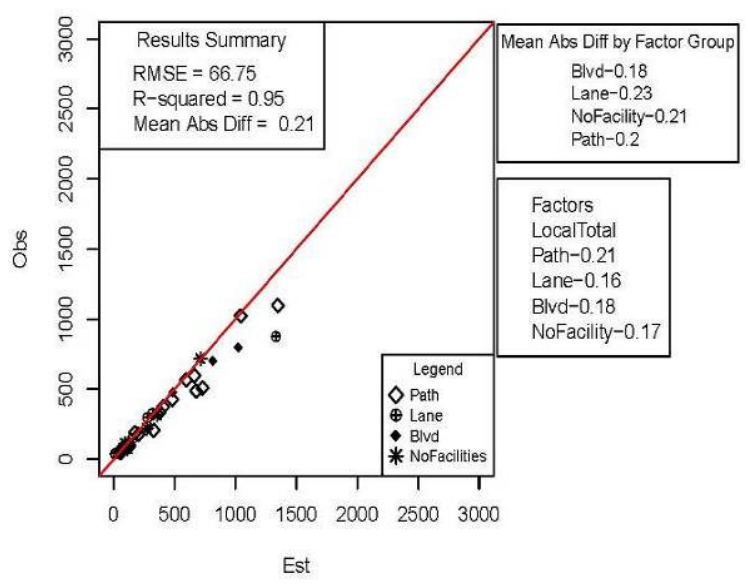

Results for NBPD PM

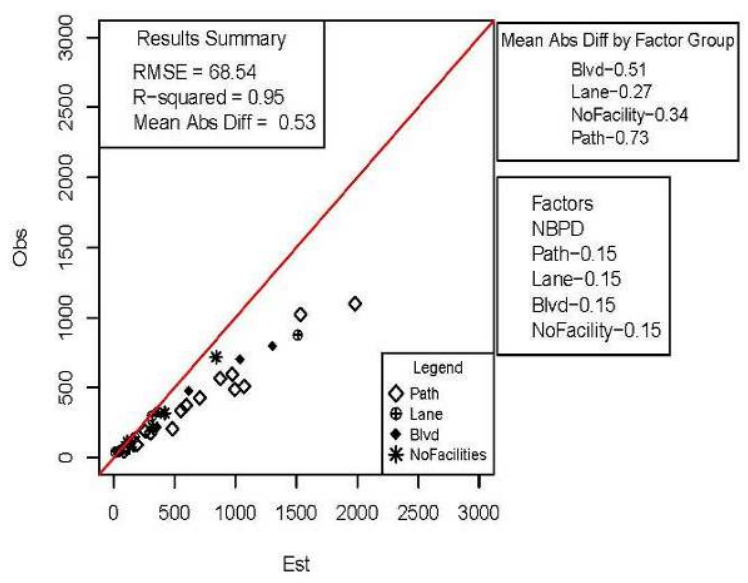


Points in the plots in Figure 3.14 that appear above the line indicate an under estimation while points on the plot below the red line indicate an over estimation. The application of each set of factors produce different outcomes. Measures of R-squared of the observed and the estimates as well as the root mean squared error and average absolute percentage difference are displayed in the top right corner of each plot. The average absolute percentage differences by factor group are also presented in the outer box in the top right hand corner of each plot. Factors used in the extrapolation process are described in the outer box to the right of each plot. The application of study area time-of-day factors using PM samples demonstrate better results compared to the morning peak which is the opposite of what is observed for the application of the NBPDP time-of-day factors which shows the AM results produce less error. The factors that produce the least error are the application of study area factors using the PM peak period factors and samples. The average absolute error is $23 \%$ which over generalizes the results somewhat since a few estimates of the factoring are very poor and skew the average. Some of this may be due to a poor initial sample used in the extrapolation calculation. One of the results with substantial error comes from location 44 (Gateway Street East I-5) where the PM study area factors produced an estimate significantly different that the observed, 12 versus 42 . With such low number of daily bicyclists it could be that this particular location is highly variable by hour and the counts used in the extrapolation calculation were not representative of the travel for that location. For 
this particular implementation of factors, the average absolute difference for locations that observed less than 100 bicyclists per day is $56 \%$ compared to $13 \%$ for locations observing 100 bicyclist or more. That being stated, there are results where daily bicyclists were less than 100 but the absolute difference was not less than $15 \%$. The results from stations with more than 100 daily bicyclists are generally better in each of the application of time-of-day factors however. Table 3.15 details the average absolute percent difference separated by count stations with more or less than 100 observed daily bicyclists.

\section{Table 3.15 - Exploring Effect of Outliers- Average Absolute \% Difference Results Comparison}

\begin{tabular}{|l|c|c|c|c|}
\hline $\begin{array}{l}\text { Factor Set } \\
\text { Used }\end{array}$ & $\begin{array}{l}\text { Average } \\
\text { Absolute } \\
\%\end{array}$ & $\begin{array}{l}\text { Average Absolute } \\
\text { \% Difference for } \\
\text { locations less } \\
\text { than 100 Daily } \\
\text { Bicyclists }\end{array}$ & $\begin{array}{l}\text { Average Absolute \% } \\
\text { Difference for } \\
\text { locations more than } \\
\text { 100 Daily Bicyclists }\end{array}$ & Period \\
\hline Study Area & $35 \%$ & $65 \%$ & $26 \%$ & AM \\
\hline Study Area & $21 \%$ & $33 \%$ & $17 \%$ & PM \\
\hline NBPDP & $52 \%$ & $102 \%$ & $38 \%$ & AM \\
\hline NBPDP & $53 \%$ & $53 \%$ & $53 \%$ & PM \\
\hline Observations & $32 \%$ & 7 & 25 & \\
\hline
\end{tabular}

Locations with less than 100 daily bicyclists might represent some kind of outlier since hourly observations can be skewed more easily by a small number of bicyclists, producing poorer results using these hourly observations in any extrapolation process. 
Table 3.16 presents the average absolute percent difference results by factor group for all estimates. For the PM results for the study area factors, absolute percent difference is less than the NBPDP factors for all factor groups. This table demonstrates that the bike boulevard (Blvd) generally resulted in the best overall result for PM study area factors with an absolute percentage difference of $18 \%$.

\section{Table 3.16 - Average Absolute \% Difference Results Comparison by Factor Group}

\begin{tabular}{|l|c|c|r|r|l|}
\hline & \multicolumn{5}{|c|}{$\begin{array}{c}\text { Average Absolute \% Difference by } \\
\text { Factor Group }\end{array}$} \\
\hline Factor Group & Blvd & Lane & \multicolumn{1}{|c|}{ No Facility } & Path & Period \\
\hline Study Area & $23 \%$ & $47 \%$ & $10 \%$ & $40 \%$ & AM \\
\hline Study Area & $18 \%$ & $23 \%$ & $21 \%$ & $20 \%$ & PM \\
\hline NBPDP & $50 \%$ & $70 \%$ & $27 \%$ & $51 \%$ & AM \\
\hline NBPDP & $51 \%$ & $27 \%$ & $34 \%$ & $73 \%$ & PM \\
\hline
\end{tabular}

In general, the estimates from each factor set implementation result in over estimation of observed counts though the study area factors over estimate by less than the NBPDP factors. Table 3.17 below demonstrates the sum of all estimates relative to the sum of observed traffic for all count stations where estimates were provided. The study area factors over estimate daily counts by $6-17 \%$ while the NBPDP factors over estimate by $32-57 \%$ when reviewed this way. These results are summarized in Table 3.15 below. 


\section{Table 3.17- Relative Levels of Over-estimation}

\begin{tabular}{|c|c|c|c|}
\hline Factors & $\begin{array}{c}\text { Sum of } \\
\text { All } \\
\text { Estimates }\end{array}$ & Observed & $\begin{array}{c}\text { Percent } \\
\text { Difference }\end{array}$ \\
\hline $\begin{array}{c}\text { Study Area } \\
\text { AM }\end{array}$ & 12,476 & 11,765 & $106 \%$ \\
\hline $\begin{array}{c}\text { Study Area } \\
\text { PM }\end{array}$ & 13,735 & 11,765 & $117 \%$ \\
\hline NBPDP AM & 15,477 & 11,765 & $132 \%$ \\
\hline NBPDP PM & 18,452 & 11,765 & $157 \%$ \\
\hline
\end{tabular}

Evaluated in a few ways, the best results come from applying the study area factors using the PM peak period. Of the 32 estimates from the PM study area factors, 18 of estimates had $15 \%$ error or less while only 6 of the NBPDP estimates were less than $15 \%$ error. More discussion of the implications of this level of error will follow the next section's testing of additional study area derived factors. 


\subsection{2 - Time-of-Day Factors from Study Area Data - Scenario 2}

The above application of factors created time-of-day expansion factors from study area data using bicycle counts collected in one season (summer) at one set of locations and applied them to count data collected in another season (fall) from a separate set of locations. This process of development and application of factors produces considerable error in some cases. Even in the best results, those using evening peak factors derived from study area data, error is as much as $59 \%$ for count locations with more than 100 daily bicyclists (up to $159 \%$ error for all locations where factors were applied). In order to decrease levels of error, an approach was developed using iterative programming in $\mathrm{R}$ to test different factors and report the level of error derived from each tested factor set looking for factors that produce the best estimates overall. These simulated factors were applied based on a factor grouping derived from the bicycle facility type where each count location was located. An additional factor grouping was added to those tried in Scenario 1 by splitting the "Path" facility into a "Path-Commute" and a "Path-Recreation" based on a review of the hourly distributions (see Figure 3.5 below and related appendix below). Path facilities with a wider evening peak period are classified as Path-Recreation while locations on paths with a more pronounced morning and afternoon peak were characterized as Path-Commute. This classification approach uses some of the methods employed by Moreno-Miranda (2012) to classify bicycle count locations except that this approach does not have weekend daily totals to inform the classification process. 
Data for this application of factors uses 24-hour counts collected on either a Tuesday or Thursday for each of the three seasons where data was available resulting in 123 days of data after cleaning out anomalous data. A process was developed in the R statistical software which iteratively tested different factors on an expanded factor grouping. The "Path" factor group used in Scenario 1 was split into two separate groups based on the hourly distribution, one for path facilities assumed to carry more commute travel and another assumed to carry more recreational travel. The process developed in $\mathrm{R}$ iterated through a vector of possible peak period factor values from 0.05 to 0.30 by increments of 0.01 and stored the results for each application of factors to determine the overall error associated with each elemental factor. By doing this, a set of factors was obtained that minimized the level of error to the greatest extent possible given the factor groupings. Figure 3.15 below describes graphically the process of the iterating through each test factor and applying the factor to each of the 123 daily counts and storing the results. After iterating through all test factors, results are reviewed to determine which factors produce the best results. The factors derived from the iterative process that produced the least amount of error are presented in Table 3.16 below. 
Figure 3.15 - Iterative Process for Determining Most Effective

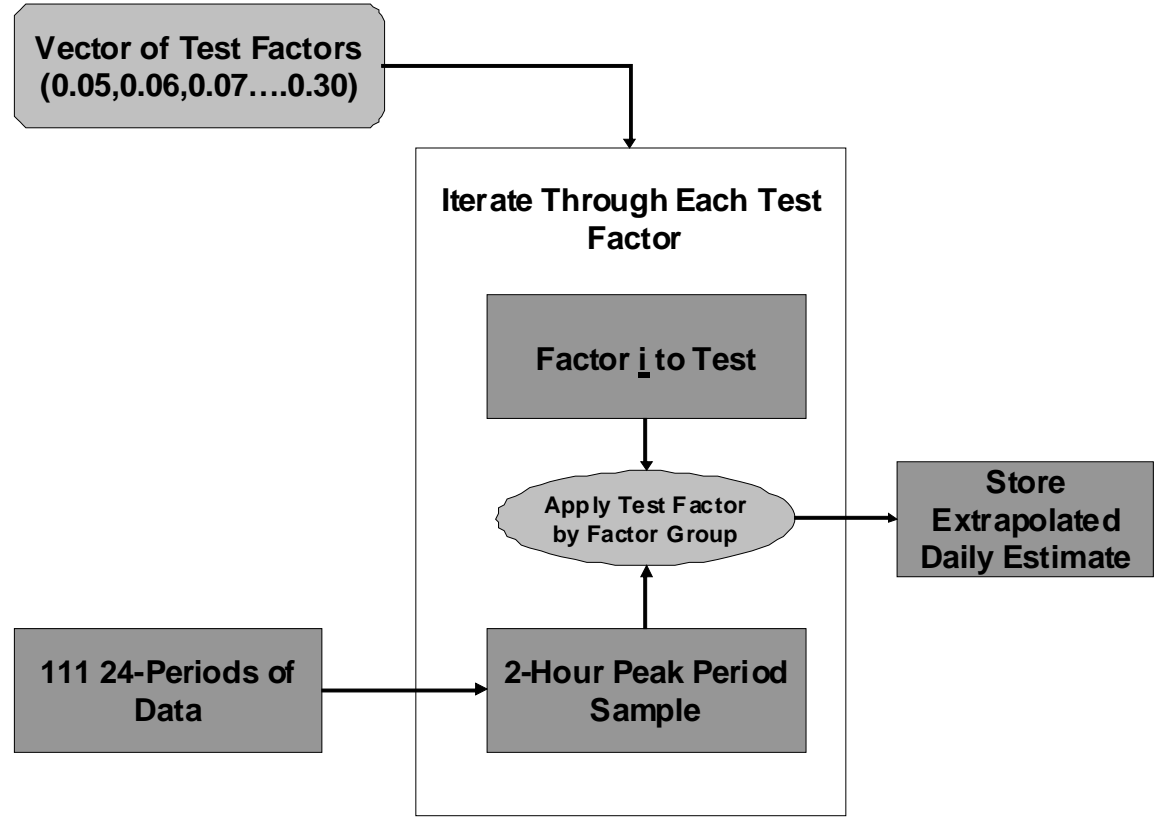

Table 3.18 - Scenario 2 Time-of-Day Extrapolation Factors

\begin{tabular}{|c|c|c|c|}
\hline \multicolumn{4}{|c|}{ Time-of-Day Factor Comparison } \\
\hline \multicolumn{2}{|l|}{ Data set } & $\begin{array}{l}\text { AM Peak Factor } \\
(7-9 \text { AM) }\end{array}$ & $\begin{array}{l}\text { PM Peak } \\
\text { Factor (4-6) }\end{array}$ \\
\hline \multirow{5}{*}{$\begin{array}{l}\text { Study Area Counts - Scenario } \\
2\end{array}$} & Path - Rec & 0.15 & 0.25 \\
\hline & Path - Commute & 0.13 & 0.21 \\
\hline & Lane & 0.15 & 0.17 \\
\hline & Blvd & 0.15 & 0.19 \\
\hline & No Facility & 0.13 & 0.19 \\
\hline
\end{tabular}


This approach to developing factors represents a completely different method of constructing factors compared to what was done in Scenario 1. It uses some simple iterative programming to determine factors that produce the least amount of error within the factor groups that were tried.

The result of this implementation is that the levels of error associated with the application of the Scenario 2 factors have been reduced. Tables $3.19-3.22$ detail the results for each location for each season where data was available. The tables also denote the day of the week since some of the data come from bicycle traffic collected on Tuesday and some on Thursday. 
Table 3.19 - Scenario 2 AM Peak Factor Results using Study Area Factors

\begin{tabular}{|c|c|c|c|c|c|c|c|c|c|c|}
\hline \multicolumn{11}{|c|}{ tudy Area - AM Factor } \\
\hline Location & Season & Observed & Estimate & Weekday & $\begin{array}{c}\% \\
\text { Difference }\end{array}$ & $\begin{array}{c}\text { Absolute } \\
\% \\
\text { Difference }\end{array}$ & $\begin{array}{c}\text { Bike } \\
\text { Facility }\end{array}$ & $\begin{array}{l}\text { Factor } \\
\text { Group }\end{array}$ & $\begin{array}{c}\text { Federal } \\
\text { Functional } \\
\text { Class }\end{array}$ & $\begin{array}{l}\text { Location } \\
\text { Id }\end{array}$ \\
\hline 13thWestAlder & Winter & 1631 & 1627 & Tuesday & $100 \%$ & $0 \%$ & Lane & Lane & $\begin{array}{l}\text { Major } \\
\text { Collector }\end{array}$ & 21 \\
\hline 16thWestAlder & Summer & 140 & 138 & Tuesday & $99 \%$ & $1 \%$ & NoFacility & NoFacility & Local & 70 \\
\hline AgateSouth18th & Summer & 438 & 433 & Thursday & $99 \%$ & $1 \%$ & Lane & Lane & Minor Arterial & 25 \\
\hline FernRidgeWestChambers & Winter & 497 & 492 & Thursday & $99 \%$ & $1 \%$ & Path & $\begin{array}{l}\text { Path- } \\
\text { Commute }\end{array}$ & Path & 95 \\
\hline FrohnmayerSouthRiver & Fall & 1691 & 1700 & Tuesday & $101 \%$ & $1 \%$ & Path & $\begin{array}{l}\text { Path- } \\
\text { Commute }\end{array}$ & Path & 6 \\
\hline SouthbankSouthGreenwayBr & Winter & 576 & 580 & Thursday & $101 \%$ & $1 \%$ & Path & Path-Rec & Path & 4 \\
\hline 12thEastLawrence & Winter & 495 & 507 & Tuesday & $102 \%$ & $2 \%$ & Blvd & Blvd & $\begin{array}{l}\text { Major } \\
\text { Collector }\end{array}$ & 96 \\
\hline 15thWestAlder & Summer & 477 & 487 & Tuesday & $102 \%$ & $2 \%$ & Blvd & Blvd & Local & 26 \\
\hline GWestMohawk & Winter & 63 & 62 & Thursday & $98 \%$ & $2 \%$ & NoFacility & NoFacility & $\begin{array}{l}\text { Major } \\
\text { Collector }\end{array}$ & 35 \\
\hline UniversityNorth24th & Fall & 220 & 215 & \begin{tabular}{|l} 
Thursday \\
\end{tabular} & $98 \%$ & $2 \%$ & NoFacility & NoFacility & Local & 15 \\
\hline 13thWestAlder & Summer & 1335 & 1300 & Tuesday & $97 \%$ & $3 \%$ & Lane & Lane & $\begin{array}{l}\text { Major } \\
\text { Collector }\end{array}$ & 21 \\
\hline 24thWestAdams & Summer & 129 & 133 & Tuesday & $103 \%$ & $3 \%$ & Lane & Lane & $\begin{array}{l}\text { Major } \\
\text { Collector }\end{array}$ & 89 \\
\hline DefazioSouthRiverEastBridge & Summer & 650 & 631 & Thursday & $97 \%$ & $3 \%$ & Path & $\begin{array}{l}\text { Path- } \\
\text { Commute }\end{array}$ & Path & 5 \\
\hline DEastPioneerPkwyPath & Winter & 74 & 77 & Tuesday & $104 \%$ & $4 \%$ & NoFacility & NoFacility & Local & 38 \\
\hline GWestMohawk & Fall & 112 & 108 & Tuesday & $96 \%$ & $4 \%$ & NoFacility & NoFacility & $\begin{array}{l}\text { Major } \\
\text { Collector }\end{array}$ & 35 \\
\hline HarlowEastl5 & Fall & 55 & 53 & \begin{tabular}{|l|} 
Thursday \\
\end{tabular} & $96 \%$ & $4 \%$ & Lane & Lane & Minor Arterial & 41 \\
\hline AgateSouthFranklin & Summer & 570 & 540 & Thursday & $95 \%$ & $5 \%$ & Lane & Lane & Minor Arterial & 23 \\
\hline FriendlySouth18th & Fall & 217 & 227 & Tuesday & $105 \%$ & $5 \%$ & Blvd & Blvd & $\begin{array}{l}\text { Major } \\
\text { Collector }\end{array}$ & 14 \\
\hline OnyxNorthFranklin & Fall & 1121 & 1177 & Tuesday & $105 \%$ & $5 \%$ & NoFacility & NoFacility & $<N A>$ & 22 \\
\hline FirEastRiverRd & Winter & 175 & 185 & Thursday & $106 \%$ & $6 \%$ & NoFacility & NoFacility & Local & 19 \\
\hline FrohnmayerSouthRiver & Summer & 1094 & 1031 & Thursday & $94 \%$ & $6 \%$ & Path & $\begin{array}{l}\text { Path- } \\
\text { Commute }\end{array}$ & Path & 6 \\
\hline GatewayBPBridgeEastl5 & Winter & 120 & 113 & \begin{tabular}{|l|} 
Thursday \\
\end{tabular} & $94 \%$ & $6 \%$ & Path & Path-Rec & Path & 40 \\
\hline HighSouth4th & Fall & 718 & 762 & Thursday & $106 \%$ & $6 \%$ & NoFacility & NoFacility & $\begin{array}{l}\text { Major } \\
\text { Collector }\end{array}$ & 48 \\
\hline 15thWestAlder & Winter & 579 & 540 & Tuesday & $93 \%$ & $7 \%$ & Blvd & Blvd & Local & 26 \\
\hline 15thWestJefferson & Fall & 798 & 740 & Tuesday & $93 \%$ & $7 \%$ & Blvd & Blvd & Local & 29 \\
\hline 15thEastAgate & Summer & 327 & 353 & Tuesday & $108 \%$ & $8 \%$ & Blvd & Blvd & Local & 24 \\
\hline 18thWestAlder & Winter & 248 & 227 & Tuesday & $92 \%$ & $8 \%$ & Lane & Lane & Minor Arterial & 46 \\
\hline
\end{tabular}




\begin{tabular}{|c|c|c|c|c|c|c|c|c|c|c|}
\hline DEastPioneerPkwyPath & Fall & 75 & 69 & Thursday & $92 \%$ & $8 \%$ & NoFacility & NoFacility & Local & 38 \\
\hline FriendlySouth18th & Winter & 192 & 207 & Tuesday & $108 \%$ & $8 \%$ & Blvd & Blvd & $\begin{array}{l}\text { Major } \\
\text { Collector }\end{array}$ & 14 \\
\hline 13thWestAlder & Summer & 1392 & 1260 & Thursday & $91 \%$ & $9 \%$ & Lane & Lane & $\begin{array}{l}\text { Major } \\
\text { Collector }\end{array}$ & 21 \\
\hline AlderNorth27th & Winter & 476 & 520 & Thursday & $109 \%$ & $9 \%$ & Blvd & Blvd & Local & 18 \\
\hline DefazioSouthRiverEastBridge & Summer & 685 & 623 & Tuesday & $91 \%$ & $9 \%$ & Path & $\begin{array}{l}\text { Path- } \\
\text { Commute }\end{array}$ & Path & 5 \\
\hline 15thWestAlder & Summer & 469 & 420 & Thursday & $90 \%$ & $10 \%$ & Blvd & Blvd & Local & 26 \\
\hline 15thWestAlder & Fall & 974 & 873 & Tuesday & $90 \%$ & $10 \%$ & Blvd & Blvd & Local & 26 \\
\hline AgateSouthFranklin & Summer & 527 & 473 & Tuesday & $90 \%$ & $10 \%$ & Lane & Lane & Minor Arterial & 23 \\
\hline AlderSouthFranklin & Summer & 345 & 380 & Thursday & $110 \%$ & $10 \%$ & Blvd & Blvd & $\begin{array}{l}\text { Major } \\
\text { Collector }\end{array}$ & 27 \\
\hline PolkNorth24th & Summer & 105 & 115 & Thursday & $110 \%$ & $10 \%$ & NoFacility & NoFacility & Local & 90 \\
\hline UniversitySouth24th & Summer & 77 & 85 & Tuesday & $110 \%$ & $10 \%$ & NoFacility & NoFacility & $\begin{array}{l}\text { Major } \\
\text { Collector }\end{array}$ & 65 \\
\hline HarlowEastl5 & Winter & 434 & 387 & Thursday & $89 \%$ & $11 \%$ & Lane & Lane & Minor Arterial & 41 \\
\hline DefazioSouthRiverEastBridge & Fall & 566 & 500 & Thursday & $88 \%$ & $12 \%$ & Path & $\begin{array}{l}\text { Path- } \\
\text { Commute }\end{array}$ & Path & 5 \\
\hline EastbankSouthOwossoBr & Winter & 136 & 120 & Tuesday & $88 \%$ & $12 \%$ & Path & Path-Rec & Path & 2 \\
\hline FernRidgeEastDanebo & Fall & 178 & 200 & Tuesday & $112 \%$ & $12 \%$ & Path & $\begin{array}{l}\text { Path- } \\
\text { Commute }\end{array}$ & Path & 9 \\
\hline HighSouth4th & Winter & 331 & 292 & Thursday & $88 \%$ & $12 \%$ & NoFacility & NoFacility & $\begin{array}{l}\text { Major } \\
\text { Collector }\end{array}$ & 48 \\
\hline DeltaBPBridgeEastGoodPasture & Winter & 61 & 53 & Tuesday & $87 \%$ & $13 \%$ & Path & Path-Rec & Path & 31 \\
\hline 24thEastFilmore & Summer & 93 & 80 & Thursday & $86 \%$ & $14 \%$ & Lane & Lane & Minor Arterial & 88 \\
\hline AgateSouth18th & Summer & 471 & 407 & Tuesday & $86 \%$ & $14 \%$ & Lane & Lane & Minor Arterial & 25 \\
\hline MonroeSouth8th & Winter & 304 & 260 & Tuesday & $86 \%$ & $14 \%$ & Blvd & Blvd & $\begin{array}{l}\text { Major } \\
\text { Collector }\end{array}$ & 11 \\
\hline 12thEastLincoln & Summer & 492 & 420 & Tuesday & $85 \%$ & $15 \%$ & Blvd & Blvd & Local & 28 \\
\hline 24thWestAdams & Summer & 117 & 100 & Thursday & $85 \%$ & $15 \%$ & Lane & Lane & $\begin{array}{l}\text { Major } \\
\text { Collector }\end{array}$ & 89 \\
\hline FernRidgeWestChambers & Winter & 537 & 454 & Tuesday & $85 \%$ & $15 \%$ & Path & $\begin{array}{l}\text { Path- } \\
\text { Commute }\end{array}$ & Path & 95 \\
\hline FrohnmayerSouthRiver & Winter & 637 & 731 & Thursday & $115 \%$ & $15 \%$ & Path & $\begin{array}{l}\text { Path- } \\
\text { Commute }\end{array}$ & Path & 6 \\
\hline GoodpastureEastDeltaHwy & Fall & 149 & 127 & Thursday & $85 \%$ & $15 \%$ & Lane & Lane & $\begin{array}{l}\text { Major } \\
\text { Collector }\end{array}$ & 1 \\
\hline RichardsonBridge & Fall & 427 & 492 & Tuesday & $115 \%$ & $15 \%$ & Path & $\begin{array}{l}\text { Path- } \\
\text { Commute }\end{array}$ & Path & 32 \\
\hline FrohnmayerSouthRiver & Summer & 1084 & 915 & Tuesday & $84 \%$ & $16 \%$ & Path & $\begin{array}{l}\text { Path- } \\
\text { Commute }\end{array}$ & Path & 6 \\
\hline AlderNorth27th & Fall & 702 & 820 & Thursday & $117 \%$ & $17 \%$ & Blvd & Blvd & Local & 18 \\
\hline AgateSouthFranklin & Fall & 563 & 460 & Tuesday & $82 \%$ & $18 \%$ & Lane & Lane & Minor Arterial & 23 \\
\hline AlderSouthFranklin & Summer & 299 & 353 & Tuesday & $118 \%$ & $18 \%$ & Blvd & Blvd & $\begin{array}{l}\text { Major } \\
\text { Collector }\end{array}$ & 27 \\
\hline HeronBridgeSouthFernRidge & Fall & 372 & 438 & Tuesday & $118 \%$ & $18 \%$ & Path & $\begin{array}{l}\text { Path- } \\
\text { Commute }\end{array}$ & Path & 10 \\
\hline
\end{tabular}




\begin{tabular}{|c|c|c|c|c|c|c|c|c|c|c|}
\hline DeltaBPBridgeEastGoodPasture & Fall & 207 & 167 & Thursday & $81 \%$ & $19 \%$ & Path & Path-Rec & Path & 31 \\
\hline 15thEastAgate & Summer & 340 & 407 & Thursday & $120 \%$ & $20 \%$ & Blvd & Blvd & Local & 24 \\
\hline OnyxNorthFranklin & Summer & 658 & 792 & Tuesday & $120 \%$ & $20 \%$ & NoFacility & NoFacility & $\langle N A>$ & 22 \\
\hline 13thWestAlder & Fall & 2602 & 2067 & Tuesday & $79 \%$ & $21 \%$ & Lane & Lane & $\begin{array}{l}\text { Major } \\
\text { Collector }\end{array}$ & 21 \\
\hline GatewayStreetEast15 & Fall & 42 & 33 & Thursday & $79 \%$ & $21 \%$ & Lane & Lane & Minor Arterial & 44 \\
\hline HeronBridgeSouthFernRidge & Winter & 342 & 415 & Tuesday & $121 \%$ & $21 \%$ & Path & $\begin{array}{l}\text { Path- } \\
\text { Commute }\end{array}$ & Path & 10 \\
\hline NorthbankEastKnickerbocker & Fall & 596 & 469 & Thursday & $79 \%$ & $21 \%$ & Path & $\begin{array}{l}\text { Path- } \\
\text { Commute }\end{array}$ & Path & 97 \\
\hline 12thWestHigh & Summer & 344 & 267 & Tuesday & $78 \%$ & $22 \%$ & Blvd & Blvd & Local & 12 \\
\hline OnyxNorthFranklin & Summer & 702 & 854 & Thursday & $122 \%$ & $22 \%$ & NoFacility & NoFacility & $\begin{array}{l}\text { Major } \\
\text { Collector } \\
\end{array}$ & 22 \\
\hline 18thWestAlder & Summer & 349 & 267 & Tuesday & $77 \%$ & $23 \%$ & Lane & Lane & Minor Arterial & 46 \\
\hline AgateSouthFranklin & Winter & 329 & 407 & Thursday & $124 \%$ & $24 \%$ & Lane & Lane & Minor Arterial & 23 \\
\hline DefazioSouthRiverEastBridge & Winter & 230 & 285 & Thursday & $124 \%$ & $24 \%$ & Path & $\begin{array}{l}\text { Path- } \\
\text { Commute }\end{array}$ & Path & 5 \\
\hline NorthbankSouthGreenwayBr & Winter & 158 & 120 & Tuesday & $76 \%$ & $24 \%$ & Path & Path-Rec & Path & 3 \\
\hline UniversitySouth18th & Summer & 213 & 162 & Tuesday & $76 \%$ & $24 \%$ & NoFacility & NoFacility & Local & 71 \\
\hline ThurstonEast58th & Winter & 36 & 27 & Thursday & $75 \%$ & $25 \%$ & Lane & Lane & Minor Arterial & 37 \\
\hline HighNorth13th & Winter & 292 & 367 & Tuesday & $126 \%$ & $26 \%$ & Lane & Lane & Minor Arterial & 30 \\
\hline OnyxNorthFranklin & Winter & 707 & 892 & Tuesday & $126 \%$ & $26 \%$ & NoFacility & NoFacility & $\begin{array}{l}\text { Major } \\
\text { Collector }\end{array}$ & 22 \\
\hline 12thEastLincoln & Summer & 513 & 373 & Thursday & $73 \%$ & $27 \%$ & Blvd & Blvd & Local & 28 \\
\hline EWEBPathEastPioneerPkwy & Fall & 47 & 60 & Tuesday & $128 \%$ & $28 \%$ & Path & Path-Rec & Path & 33 \\
\hline FirEastRiverRd & Fall & 319 & 231 & Thursday & $72 \%$ & $28 \%$ & NoFacility & NoFacility & Local & 19 \\
\hline UniversitySouth18th & Summer & 203 & 146 & Thursday & $72 \%$ & $28 \%$ & NoFacility & NoFacility & Local & 71 \\
\hline 15thEastAgate & Fall & 1021 & 713 & Thursday & $70 \%$ & $30 \%$ & Blvd & Blvd & Local & 24 \\
\hline 15thEastAgate & Winter & 814 & 573 & Tuesday & $70 \%$ & $30 \%$ & Blvd & Blvd & Local & 24 \\
\hline 42ndNorthCommercial & Winter & 189 & 133 & Thursday & $70 \%$ & $30 \%$ & Lane & Lane & Minor Arterial & 45 \\
\hline AgateSouth18th & Winter & 295 & 207 & Thursday & $70 \%$ & $30 \%$ & Lane & Lane & Minor Arterial & 25 \\
\hline ClearwaterPath & Winter & 10 & 13 & Thursday & $130 \%$ & $30 \%$ & Path & Path-Rec & Path & 98 \\
\hline HarrisSouth18th & Summer & 121 & 85 & Thursday & $70 \%$ & $30 \%$ & NoFacility & NoFacility & $\begin{array}{l}\text { Major } \\
\text { Collector }\end{array}$ & 73 \\
\hline 16thWestAlder & Summer & 167 & 115 & Thursday & $69 \%$ & $31 \%$ & NoFacility & NoFacility & Local & 70 \\
\hline 24thEastFilmore & Summer & 101 & 133 & Tuesday & $132 \%$ & $32 \%$ & Lane & Lane & Minor Arterial & 88 \\
\hline AlderSouthFranklin & Winter & 293 & 387 & Tuesday & $132 \%$ & $32 \%$ & Blvd & Blvd & $\begin{array}{l}\text { Major } \\
\text { Collector } \\
\end{array}$ & 27 \\
\hline EWEBPathEast5th & Fall & 76 & 100 & Tuesday & $132 \%$ & $32 \%$ & Path & Path-Rec & Path & 34 \\
\hline 13thEastHigh & Fall & 623 & 827 & Thursday & $133 \%$ & $33 \%$ & Lane & Lane & $\langle\mathrm{NA}\rangle$ & 52 \\
\hline MonroeSouth8th & Fall & 478 & 320 & Thursday & $67 \%$ & $33 \%$ & Blvd & Blvd & $\begin{array}{l}\text { Major } \\
\text { Collector }\end{array}$ & 11 \\
\hline SouthbankSouthGreenwayBr & Fall & 1098 & 740 & Tuesday & $67 \%$ & $33 \%$ & Path & Path-Rec & Path & 4 \\
\hline GatewayStreetEast15 & Winter & 82 & 53 & Thursday & $65 \%$ & $35 \%$ & Lane & Lane & Minor Arterial & 44 \\
\hline PioneerPkwySouthQ & Winter & 123 & 80 & Tuesday & $65 \%$ & $35 \%$ & Path & Path-Rec & Path & 39 \\
\hline 11thWestAlder & Summer & 180 & 113 & Thursday & $63 \%$ & $37 \%$ & Lane & Lane & Minor Arterial & 72 \\
\hline
\end{tabular}




\begin{tabular}{|c|c|c|c|c|c|c|c|c|c|c|}
\hline 12thWestHigh & Fall & 466 & 293 & Tuesday & $63 \%$ & $37 \%$ & Blvd & Blvd & Local & 12 \\
\hline NorthbankWestDeltaHwy & Winter & 147 & 92 & Tuesday & $63 \%$ & $37 \%$ & Path & \begin{tabular}{|l|} 
Path- \\
Commute
\end{tabular} & Path & 16 \\
\hline UniversityNorth24th & Winter & 184 & 115 & Thursday & $62 \%$ & $38 \%$ & NoFacility & NoFacility & Local & 15 \\
\hline PearlSouth19th & Fall & 119 & 73 & Thursday & $61 \%$ & $39 \%$ & Lane & Lane & \begin{tabular}{|l} 
Major \\
Collector
\end{tabular} & 17 \\
\hline NorthbankSouthGreenwayBr & Fall & 509 & 307 & Tuesday & $60 \%$ & $40 \%$ & Path & Path-Rec & Path & 3 \\
\hline 18thWestAlder & Fall & 646 & 373 & Thursday & $58 \%$ & $42 \%$ & Lane & Lane & Minor Arterial & 46 \\
\hline 15thWestJefferson & Winter & 348 & 193 & \begin{tabular}{|l|} 
Thursday \\
\end{tabular} & $55 \%$ & $45 \%$ & Blvd & Blvd & Local & 29 \\
\hline 18thWestAlder & Summer & 352 & 193 & Thursday & $55 \%$ & $45 \%$ & Lane & Lane & Minor Arterial & 46 \\
\hline RichardsonBridge & Winter & 387 & 562 & Tuesday & $145 \%$ & $45 \%$ & Path & \begin{tabular}{|l|} 
Path- \\
Commute
\end{tabular} & Path & 32 \\
\hline 32ndSouthOregon & Winter & 36 & 53 & Thursday & $147 \%$ & $47 \%$ & Lane & Lane & Minor Arterial & 36 \\
\hline AmazonPathNorth24th & Fall & 1023 & 1508 & Thursday & $147 \%$ & $47 \%$ & Path & \begin{tabular}{|l|} 
Path- \\
Commute
\end{tabular} & Path & 8 \\
\hline PioneerPkwySouthQ & Fall & 190 & 100 & Tuesday & $53 \%$ & $47 \%$ & Path & Path-Rec & Path & 39 \\
\hline WillametteNorth15th & Fall & 330 & 173 & Tuesday & $52 \%$ & $48 \%$ & Lane & Lane & Minor Arterial & 13 \\
\hline 11thWestAlder & Summer & 209 & 107 & Tuesday & $51 \%$ & $49 \%$ & Lane & Lane & Minor Arterial & 72 \\
\hline 12thWestHigh & Winter & 393 & 200 & Tuesday & $51 \%$ & $49 \%$ & Blvd & Blvd & Local & 12 \\
\hline EastbankSouthOwossoBr & Fall & 488 & 247 & Thursday & $51 \%$ & $49 \%$ & Path & Path-Rec & \begin{tabular}{|l|} 
Path \\
\end{tabular} & 2 \\
\hline HighNorth13th & Fall & 299 & 447 & Tuesday & $149 \%$ & $49 \%$ & Lane & Lane & Minor Arterial & 30 \\
\hline PolkNorth24th & Summer & 88 & 131 & Tuesday & $149 \%$ & $49 \%$ & NoFacility & NoFacility & Local & 90 \\
\hline 12thWestHigh & Summer & 372 & 187 & \begin{tabular}{|l} 
Thursday \\
\end{tabular} & $50 \%$ & $50 \%$ & Blvd & Blvd & Local & 12 \\
\hline NorthbankWestDeltaHwy & Fall & 338 & 162 & Thursday & $48 \%$ & $52 \%$ & Path & \begin{tabular}{|l|} 
Path- \\
Commute
\end{tabular} & Path & 16 \\
\hline AmazonPathNorth24th & Winter & 538 & 831 & Thursday & $154 \%$ & $54 \%$ & Path & \begin{tabular}{|l|} 
Path- \\
Commute
\end{tabular} & Path & 8 \\
\hline GatewayBPBridgeEastl5 & Fall & 95 & 147 & Thursday & $155 \%$ & $55 \%$ & Path & Path-Rec & Path & 40 \\
\hline 32ndSouthOregon & Fall & 878 & 380 & Thursday & $43 \%$ & $57 \%$ & Lane & Lane & Minor Arterial & 36 \\
\hline UniversitySouth24th & Summer & 68 & 108 & Thursday & $159 \%$ & $59 \%$ & NoFacility & NoFacility & Major & 65 \\
\hline FernRidgeEastDanebo & Winter & 144 & 231 & Tuesday & $160 \%$ & $60 \%$ & Path & \begin{tabular}{|l|} 
Path- \\
Commute \\
\end{tabular} & Path & 9 \\
\hline WillametteNorth15th & Winter & 311 & 80 & Tuesday & $26 \%$ & $74 \%$ & Lane & \begin{tabular}{|l} 
Lane \\
\end{tabular} & Minor Arterial & 13 \\
\hline DefazioNorthRiverWestBridge & Winter & 104 & 200 & Thursday & $192 \%$ & $92 \%$ & Path & \begin{tabular}{|l} 
Path- \\
Commute
\end{tabular} & Path & 47 \\
\hline ThurstonEast58th & Fall & 44 & 100 & Thursday & $227 \%$ & $127 \%$ & Lane & \begin{tabular}{|l} 
Lane \\
\end{tabular} & Minor Arterial & 37 \\
\hline
\end{tabular}


Table 3.20 - Scenario 2 PM Peak Factor Results using Study Area Factors

\begin{tabular}{|c|c|c|c|c|c|c|c|c|c|c|}
\hline \multicolumn{11}{|c|}{ Study Area - PM Factor } \\
\hline Location & Season & Observed & Estimate & Weekday & $\begin{array}{c}\% \\
\text { Difference }\end{array}$ & \begin{tabular}{|c} 
Absolute \\
$\%$ \\
Difference
\end{tabular} & $\begin{array}{c}\text { Bike } \\
\text { Facility }\end{array}$ & $\begin{array}{l}\text { Factor } \\
\text { Group } \\
\end{array}$ & $\begin{array}{c}\text { Federal } \\
\text { Functional } \\
\text { Class }\end{array}$ & $\begin{array}{l}\text { Location } \\
\text { Id }\end{array}$ \\
\hline FernRidgeWestChambers & Winter & 537 & 538 & Tuesday & $100 \%$ & $0 \%$ & Path & $\begin{array}{l}\text { Path- } \\
\text { Commute }\end{array}$ & Path & 95 \\
\hline FrohnmayerSouthRiver & Fall & 1691 & 1686 & Tuesday & $100 \%$ & $0 \%$ & Path & $\begin{array}{l}\text { Path- } \\
\text { Commute }\end{array}$ & Path & 6 \\
\hline 18thWestAlder & Fall & 646 & 641 & Thursday & $99 \%$ & $1 \%$ & Lane & Lane & Minor Arterial & 46 \\
\hline FirEastRiverRd & Fall & 319 & 316 & \begin{tabular}{|l} 
Thursday \\
\end{tabular} & $99 \%$ & $1 \%$ & NoFacility & NoFacility & Local & 19 \\
\hline GoodpastureEastDeltaHwy & Fall & 149 & 147 & Thursday & $99 \%$ & $1 \%$ & \begin{tabular}{|l} 
Lane \\
\end{tabular} & Lane & $\langle N A>$ & 1 \\
\hline AmazonPathNorth24th & Fall & 1023 & 1043 & Thursday & $102 \%$ & $2 \%$ & Path & $\begin{array}{l}\text { Path- } \\
\text { Commute }\end{array}$ & Path & 8 \\
\hline EWEBPathEastPioneerPkwy & Fall & 47 & 48 & Tuesday & $102 \%$ & $2 \%$ & Path & Path-Rec & Path & 33 \\
\hline FirEastRiverRd & Winter & 175 & 179 & Thursday & $102 \%$ & $2 \%$ & NoFacility & NoFacility & Local & 19 \\
\hline FriendlySouth18th & Winter & 192 & 195 & Tuesday & $102 \%$ & $2 \%$ & Blvd & Blvd & $\begin{array}{l}\text { Major } \\
\text { Collector }\end{array}$ & 14 \\
\hline OnyxNorthFranklin & Summer & 658 & 674 & Tuesday & $102 \%$ & $2 \%$ & NoFacility & NoFacility & $<\mathrm{NA}>$ & 22 \\
\hline EastbankSouthOwossoBr & Winter & 136 & 132 & Tuesday & $97 \%$ & $3 \%$ & \begin{tabular}{|l} 
Path \\
\end{tabular} & Path-Rec & Path & 2 \\
\hline FrohnmayerSouthRiver & Summer & 1084 & 1048 & Tuesday & $97 \%$ & $3 \%$ & Path & $\begin{array}{l}\text { Path- } \\
\text { Commute }\end{array}$ & Path & 6 \\
\hline MonroeSouth8th & Fall & 478 & 463 & Thursday & $97 \%$ & $3 \%$ & Blvd & Blvd & $\begin{array}{l}\text { Major } \\
\text { Collector }\end{array}$ & 11 \\
\hline SouthbankSouthGreenwayBr & Fall & 1098 & 1132 & Tuesday & $103 \%$ & $3 \%$ & Path & Path-Rec & Path & 4 \\
\hline ThurstonEast58th & Winter & 36 & 35 & Thursday & $97 \%$ & $3 \%$ & Lane & Lane & Minor Arterial & 37 \\
\hline AgateSouthFranklin & Winter & 329 & 341 & Thursday & $104 \%$ & $4 \%$ & Lane & Lane & Minor Arterial & 23 \\
\hline HarlowEast15 & Winter & 434 & 453 & Thursday & $104 \%$ & $4 \%$ & Lane & Lane & Minor Arterial & 41 \\
\hline HarrisSouth18th & Summer & 121 & 116 & Thursday & $96 \%$ & $4 \%$ & NoFacility & NoFacility & $\begin{array}{l}\text { Major } \\
\text { Collector }\end{array}$ & 73 \\
\hline PearlSouth19th & Fall & 119 & 124 & Thursday & $104 \%$ & $4 \%$ & Lane & Lane & $\begin{array}{l}\text { Major } \\
\text { Collector }\end{array}$ & 17 \\
\hline DefazioSouthRiverEastBridge & Fall & 566 & 595 & Thursday & $105 \%$ & $5 \%$ & Path & $\begin{array}{l}\text { Path- } \\
\text { Commute }\end{array}$ & Path & 5 \\
\hline UniversityNorth24th & Winter & 184 & 174 & Thursday & $95 \%$ & $5 \%$ & NoFacility & NoFacility & Local & 15 \\
\hline 15thWestAlder & Summer & 469 & 442 & $\begin{array}{l}\text { Thursday } \\
\end{array}$ & $94 \%$ & $6 \%$ & Blvd & Blvd & Local & 26 \\
\hline NorthbankSouthGreenwayBr & Winter & 158 & 168 & Tuesday & $106 \%$ & $6 \%$ & Path & Path-Rec & Path & 3 \\
\hline NorthbankWestDeltaHwy & Winter & 147 & 138 & Tuesday & $94 \%$ & $6 \%$ & Path & $\begin{array}{l}\text { Path- } \\
\text { Commute }\end{array}$ & Path & 16 \\
\hline UniversitySouth18th & Summer & 213 & 200 & Tuesday & $94 \%$ & $6 \%$ & NoFacility & NoFacility & Local & 71 \\
\hline UniversitySouth18th & Summer & 203 & 216 & Thursday & $106 \%$ & $6 \%$ & NoFacility & NoFacility & Local & 71 \\
\hline 15thEastAgate & Summer & 340 & 316 & $\begin{array}{l}\text { Thursday } \\
\end{array}$ & $93 \%$ & $7 \%$ & Blvd & Blvd & Local & 24 \\
\hline 15thWestAlder & Fall & 974 & 905 & Tuesday & $93 \%$ & $7 \%$ & Blvd & Blvd & Local & 26 \\
\hline 18thWestAlder & Summer & 349 & 324 & Tuesday & $93 \%$ & $7 \%$ & Lane & Lane & Minor Arterial & 46 \\
\hline
\end{tabular}




\begin{tabular}{|c|c|c|c|c|c|c|c|c|c|c|}
\hline MonroeSouth8th & Winter & 304 & 326 & Tuesday & $107 \%$ & $7 \%$ & Blvd & Blvd & $\begin{array}{l}\text { Major } \\
\text { Collector }\end{array}$ & 11 \\
\hline WillametteNorth15th & Fall & 330 & 306 & Tuesday & $93 \%$ & $7 \%$ & Lane & Lane & Minor Arterial & 13 \\
\hline 24thEastFilmore & Summer & 93 & 100 & Thursday & $108 \%$ & $8 \%$ & Lane & Lane & Minor Arterial & 88 \\
\hline AlderSouthFranklin & Summer & 345 & 316 & Thursday & $92 \%$ & $8 \%$ & Blvd & Blvd & $\begin{array}{l}\text { Major } \\
\text { Collector }\end{array}$ & 27 \\
\hline DefazioSouthRiverEastBridge & Summer & 685 & 743 & Tuesday & $108 \%$ & $8 \%$ & Path & $\begin{array}{l}\text { Path- } \\
\text { Commute }\end{array}$ & Path & 5 \\
\hline 12thWestHigh & Summer & 344 & 374 & Tuesday & $109 \%$ & $9 \%$ & Blvd & Blvd & Local & 12 \\
\hline 15thWestAlder & Winter & 579 & 526 & Tuesday & $91 \%$ & $9 \%$ & Blvd & Blvd & Local & 26 \\
\hline 42ndNorthCommercial & Winter & 189 & 206 & \begin{tabular}{|l|} 
Thursday \\
\end{tabular} & $109 \%$ & $9 \%$ & Lane & Lane & Minor Arterial & 45 \\
\hline AgateSouth18th & Summer & 438 & 476 & \begin{tabular}{|l|} 
Thursday \\
\end{tabular} & $109 \%$ & $9 \%$ & Lane & Lane & Minor Arterial & 25 \\
\hline AgateSouthFranklin & Fall & 563 & 612 & \begin{tabular}{|l|} 
Tuesday \\
\end{tabular} & $109 \%$ & $9 \%$ & Lane & Lane & Minor Arterial & 23 \\
\hline DefazioNorthRiverWestBridge & Winter & 104 & 95 & Thursday & $91 \%$ & $9 \%$ & Path & $\begin{array}{l}\text { Path- } \\
\text { Commute }\end{array}$ & Path & 47 \\
\hline FrohnmayerSouthRiver & Summer & 1094 & 1190 & Thursday & $109 \%$ & $9 \%$ & Path & $\begin{array}{l}\text { Path- } \\
\text { Commute }\end{array}$ & Path & 6 \\
\hline HeronBridgeSouthFernRidge & Fall & 372 & 405 & Tuesday & $109 \%$ & $9 \%$ & Path & $\begin{array}{l}\text { Path- } \\
\text { Commute }\end{array}$ & Path & 10 \\
\hline OnyxNorthFranklin & Summer & 702 & 642 & \begin{tabular}{|l|} 
Thursday \\
\end{tabular} & $91 \%$ & $9 \%$ & NoFacility & NoFacility & $<N A>$ & 22 \\
\hline OnyxNorthFranklin & Winter & 707 & 768 & Tuesday & $109 \%$ & $9 \%$ & NoFacility & NoFacility & $<N A$ & 22 \\
\hline 15thEastAgate & Summer & 327 & 295 & Tuesday & $90 \%$ & $10 \%$ & Blvd & Blvd & Local & 24 \\
\hline GatewayBPBridgeEast15 & Winter & 120 & 108 & \begin{tabular}{|l} 
Thursday \\
\end{tabular} & $90 \%$ & $10 \%$ & Path & Path-Rec & Path & 40 \\
\hline UniversityNorth24th & Fall & 220 & 242 & \begin{tabular}{|l|} 
Thursday \\
\end{tabular} & $110 \%$ & $10 \%$ & NoFacility & NoFacility & Local & 15 \\
\hline 12thEastLincoln & Summer & 492 & 437 & Tuesday & $89 \%$ & $11 \%$ & Blvd & Blvd & Local & 28 \\
\hline AlderNorth27th & Fall & 702 & 779 & Thursday & $111 \%$ & $11 \%$ & Blvd & Blvd & Local & 18 \\
\hline HighNorth13th & Fall & 299 & 265 & Tuesday & $89 \%$ & $11 \%$ & Lane & Lane & Minor Arterial & 30 \\
\hline NorthbankEastKnickerbocker & Fall & 596 & 662 & Thursday & $111 \%$ & $11 \%$ & Path & $\begin{array}{l}\text { Path- } \\
\text { Commute }\end{array}$ & Path & 97 \\
\hline NorthbankWestDeltaHwy & Fall & 338 & 376 & Thursday & $111 \%$ & $11 \%$ & Path & $\begin{array}{l}\text { Path- } \\
\text { Commute }\end{array}$ & Path & 16 \\
\hline 12thWestHigh & Fall & 466 & 521 & Tuesday & $112 \%$ & $12 \%$ & Blvd & Blvd & Local & 12 \\
\hline 12thWestHigh & Winter & 393 & 442 & Tuesday & $112 \%$ & $12 \%$ & Blvd & Blvd & Local & 12 \\
\hline HighSouth4th & Fall & 718 & 632 & Thursday & $88 \%$ & $12 \%$ & NoFacility & NoFacility & $\begin{array}{l}\text { Major } \\
\text { Collector }\end{array}$ & 48 \\
\hline OnyxNorthFranklin & Fall & 1121 & 984 & \begin{tabular}{|l} 
Tuesday \\
\end{tabular} & $88 \%$ & $12 \%$ & NoFacility & NoFacility & $<N A>$ & 22 \\
\hline AlderNorth27th & Winter & 476 & 416 & \begin{tabular}{|l|} 
Thursday \\
\end{tabular} & $87 \%$ & $13 \%$ & Blvd & Blvd & Local & 18 \\
\hline FrohnmayerSouthRiver & Winter & 637 & 552 & Thursday & $87 \%$ & $13 \%$ & Path & $\begin{array}{l}\text { Path- } \\
\text { Commute }\end{array}$ & Path & 6 \\
\hline GatewayStreetEast15 & Winter & 82 & 71 & \begin{tabular}{|l} 
Thursday \\
\end{tabular} & $87 \%$ & $13 \%$ & Lane & Lane & Minor Arterial & 44 \\
\hline RichardsonBridge & Fall & 427 & 481 & Tuesday & $113 \%$ & $13 \%$ & Path & $\begin{array}{l}\text { Path- } \\
\text { Commute }\end{array}$ & Path & 32 \\
\hline WillametteNorth15th & Winter & 311 & 271 & Tuesday & $87 \%$ & $13 \%$ & Lane & Lane & Minor Arterial & 13 \\
\hline 15thWestAlder & Summer & 477 & 411 & Tuesday & $86 \%$ & $14 \%$ & Blvd & Blvd & Local & 26 \\
\hline DefazioSouthRiverEastBridge & Summer & 650 & 557 & Thursday & $86 \%$ & $14 \%$ & Path & $\begin{array}{l}\text { Path- } \\
\text { Commute }\end{array}$ & Path & 5 \\
\hline
\end{tabular}




\begin{tabular}{|c|c|c|c|c|c|c|c|c|c|c|}
\hline GatewayBPBridgeEast15 & Fall & 95 & 108 & Thursday & $114 \%$ & $14 \%$ & Path & Path-Rec & Path & 40 \\
\hline 11thWestAlder & Summer & 209 & 241 & Tuesday & $115 \%$ & $15 \%$ & Lane & Lane & Minor Arterial & 72 \\
\hline DefazioSouthRiverEastBridge & Winter & 230 & 195 & Thursday & $85 \%$ & $15 \%$ & Path & $\begin{array}{l}\text { Path- } \\
\text { Commute }\end{array}$ & Path & 5 \\
\hline DeltaBPBridgeEastGoodPasture & Winter & 61 & 52 & Tuesday & $85 \%$ & $15 \%$ & Path & Path-Rec & Path & 31 \\
\hline FernRidgeEastDanebo & Fall & 178 & 205 & Tuesday & $115 \%$ & $15 \%$ & Path & $\begin{array}{l}\text { Path- } \\
\text { Commute }\end{array}$ & Path & 9 \\
\hline HarlowEast15 & Fall & 55 & 47 & Thursday & $85 \%$ & $15 \%$ & Lane & Lane & Minor Arterial & 41 \\
\hline HeronBridgeSouthFernRidge & Winter & 342 & 290 & Tuesday & $85 \%$ & $15 \%$ & Path & $\begin{array}{l}\text { Path- } \\
\text { Commute }\end{array}$ & Path & 10 \\
\hline 15thWestJefferson & Winter & 348 & 405 & Thursday & $116 \%$ & $16 \%$ & Blvd & Blvd & Local & 29 \\
\hline 18thWestAlder & Summer & 352 & 294 & Thursday & $84 \%$ & $16 \%$ & Lane & Lane & Minor Arterial & 46 \\
\hline AgateSouthFranklin & Summer & 527 & 612 & Tuesday & $116 \%$ & $16 \%$ & Lane & Lane & Minor Arterial & 23 \\
\hline AmazonPathNorth24th & Winter & 538 & 452 & Thursday & $84 \%$ & $16 \%$ & Path & $\begin{array}{l}\text { Path- } \\
\text { Commute }\end{array}$ & Path & 8 \\
\hline EastbankSouthOwossoBr & Fall & 488 & 568 & Thursday & $116 \%$ & $16 \%$ & Path & Path-Rec & Path & 2 \\
\hline 12thEastLawrence & Winter & 495 & 411 & Tuesday & $83 \%$ & $17 \%$ & Blvd & Blvd & $<\mathrm{NA}>$ & 96 \\
\hline 12thEastLincoln & Summer & 513 & 426 & Thursday & $83 \%$ & $17 \%$ & Blvd & Blvd & Local & 28 \\
\hline 13thWestAlder & Summer & 1335 & 1112 & Tuesday & $83 \%$ & $17 \%$ & Lane & Lane & $\begin{array}{l}\text { Major } \\
\text { Collector }\end{array}$ & 21 \\
\hline 18thWestAlder & Winter & 248 & 206 & Tuesday & $83 \%$ & $17 \%$ & Lane & Lane & Minor Arterial & 46 \\
\hline AlderSouthFranklin & Summer & 299 & 247 & Tuesday & $83 \%$ & $17 \%$ & Blvd & Blvd & $\begin{array}{l}\text { Major } \\
\text { Collector }\end{array}$ & 27 \\
\hline 13thWestAlder & Fall & 2602 & 2129 & Tuesday & $82 \%$ & $18 \%$ & Lane & Lane & $\begin{array}{l}\text { Major } \\
\text { Collector }\end{array}$ & 21 \\
\hline 24thWestAdams & Summer & 129 & 106 & Tuesday & $82 \%$ & $18 \%$ & Lane & Lane & $\begin{array}{l}\text { Major } \\
\text { Collector }\end{array}$ & 89 \\
\hline FernRidgeWestChambers & Winter & 497 & 410 & Thursday & $82 \%$ & $18 \%$ & Path & $\begin{array}{l}\text { Path- } \\
\text { Commute }\end{array}$ & Path & 95 \\
\hline RichardsonBridge & Winter & 387 & 457 & Tuesday & $118 \%$ & $18 \%$ & Path & $\begin{array}{l}\text { Path- } \\
\text { Commute }\end{array}$ & Path & 32 \\
\hline 32ndSouthOregon & Winter & 36 & 29 & Thursday & $81 \%$ & $19 \%$ & Lane & Lane & Minor Arterial & 36 \\
\hline PioneerPkwySouthQ & Winter & 123 & 100 & Tuesday & $81 \%$ & $19 \%$ & Path & Path-Rec & Path & 39 \\
\hline ClearwaterPath & Winter & 10 & 12 & Thursday & $120 \%$ & $20 \%$ & Path & Path-Rec & Path & 98 \\
\hline DEastPioneerPkwyPath & Winter & 74 & 89 & Tuesday & $120 \%$ & $20 \%$ & NoFacility & NoFacility & Local & 38 \\
\hline NorthbankSouthGreenwayBr & Fall & 509 & 612 & Tuesday & $120 \%$ & $20 \%$ & Path & Path-Rec & Path & 3 \\
\hline 16thWestAlder & Summer & 140 & 111 & Tuesday & $79 \%$ & $21 \%$ & NoFacility & NoFacility & Local & 70 \\
\hline 24thWestAdams & Summer & 117 & 141 & Thursday & $121 \%$ & $21 \%$ & Lane & Lane & $\begin{array}{l}\text { Major } \\
\text { Collector }\end{array}$ & 89 \\
\hline 12thWestHigh & Summer & 372 & 453 & Thursday & $122 \%$ & $22 \%$ & Blvd & Blvd & Local & 12 \\
\hline PioneerPkwySouthQ & Fall & 190 & 148 & Tuesday & $78 \%$ & $22 \%$ & Path & Path-Rec & Path & 39 \\
\hline 15thWestJefferson & Fall & 798 & 979 & Tuesday & $123 \%$ & $23 \%$ & Blvd & Blvd & Local & 29 \\
\hline AgateSouthFranklin & Summer & 570 & 700 & Thursday & $123 \%$ & $23 \%$ & Lane & Lane & Minor Arterial & 23 \\
\hline PolkNorth24th & Summer & 88 & 68 & Tuesday & $77 \%$ & $23 \%$ & NoFacility & NoFacility & Local & 90 \\
\hline FriendlySouth18th & Fall & 217 & 268 & Tuesday & $124 \%$ & $24 \%$ & Blvd & Blvd & $\begin{array}{l}\text { Major } \\
\text { Collector }\end{array}$ & 14 \\
\hline
\end{tabular}




\begin{tabular}{|c|c|c|c|c|c|c|c|c|c|c|}
\hline SouthbankSouthGreenwayBr & Winter & 576 & 436 & Thursday & $76 \%$ & $24 \%$ & Path & Path-Rec & Path & 4 \\
\hline 24thEastFilmore & Summer & 101 & 76 & Tuesday & $75 \%$ & $25 \%$ & Lane & Lane & Minor Arterial & 88 \\
\hline AlderSouthFranklin & Winter & 293 & 221 & Tuesday & $75 \%$ & $25 \%$ & Blvd & Blvd & $\begin{array}{l}\text { Major } \\
\text { Collector }\end{array}$ & 27 \\
\hline GWestMohawk & Fall & 112 & 84 & Tuesday & $75 \%$ & $25 \%$ & NoFacility & NoFacility & $\begin{array}{l}\text { Major } \\
\text { Collector }\end{array}$ & 35 \\
\hline PolkNorth24th & Summer & 105 & 79 & Thursday & $75 \%$ & $25 \%$ & NoFacility & NoFacility & Local & 90 \\
\hline 13thWestAlder & Winter & 1631 & 1200 & Tuesday & $74 \%$ & $26 \%$ & Lane & Lane & $\begin{array}{l}\text { Major } \\
\text { Collector }\end{array}$ & 21 \\
\hline EWEBPathEast5th & Fall & 76 & 56 & Tuesday & $74 \%$ & $26 \%$ & Path & Path-Rec & Path & 34 \\
\hline 11thWestAlder & Summer & 180 & 229 & Thursday & $127 \%$ & $27 \%$ & Lane & Lane & Minor Arterial & 72 \\
\hline 15thEastAgate & Winter & 814 & 589 & Tuesday & $72 \%$ & $28 \%$ & Blvd & Blvd & Local & 24 \\
\hline 16thWestAlder & Summer & 167 & 121 & Thursday & $72 \%$ & $28 \%$ & NoFacility & NoFacility & Local & 70 \\
\hline 13thWestAlder & Summer & 1392 & 988 & Thursday & $71 \%$ & $29 \%$ & Lane & Lane & $\begin{array}{l}\text { Major } \\
\text { Collector }\end{array}$ & 21 \\
\hline HighSouth4th & Winter & 331 & 232 & Thursday & $70 \%$ & $30 \%$ & NoFacility & NoFacility & $\begin{array}{l}\text { Major } \\
\text { Collector }\end{array}$ & 48 \\
\hline AgateSouth18th & Summer & 471 & 624 & Tuesday & $132 \%$ & $32 \%$ & Lane & Lane & Minor Arterial & 25 \\
\hline DEastPioneerPkwyPath & Fall & 75 & 100 & Thursday & $133 \%$ & $33 \%$ & NoFacility & NoFacility & Local & 38 \\
\hline DeltaBPBridgeEastGoodPasture & Fall & 207 & 276 & Thursday & $133 \%$ & $33 \%$ & Path & Path-Rec & Path & 31 \\
\hline 15thEastAgate & Fall & 1021 & 674 & Thursday & $66 \%$ & $34 \%$ & Blvd & Blvd & Local & 24 \\
\hline ThurstonEast58th & Fall & 44 & 29 & Thursday & $66 \%$ & $34 \%$ & Lane & Lane & Minor Arterial & 37 \\
\hline AgateSouth18th & Winter & 295 & 400 & Thursday & $136 \%$ & $36 \%$ & Lane & Lane & Minor Arterial & 25 \\
\hline 13thEastHigh & Fall & 623 & 394 & Thursday & $63 \%$ & $37 \%$ & Lane & Lane & $\langle\mathrm{NA}\rangle$ & 52 \\
\hline HighNorth13th & Winter & 292 & 176 & Tuesday & $60 \%$ & $40 \%$ & Lane & Lane & Minor Arterial & 30 \\
\hline FernRidgeEastDanebo & Winter & 144 & 205 & Tuesday & $142 \%$ & $42 \%$ & Path & $\begin{array}{l}\text { Path- } \\
\text { Commute }\end{array}$ & Path & 9 \\
\hline UniversitySouth24th & Summer & 77 & 111 & Tuesday & $144 \%$ & $44 \%$ & NoFacility & NoFacility & $\langle N A>$ & 65 \\
\hline 32ndSouthOregon & Fall & 878 & 1271 & Thursday & $145 \%$ & $45 \%$ & Lane & Lane & Minor Arterial & 36 \\
\hline GWestMohawk & Winter & 63 & 32 & Thursday & $51 \%$ & $49 \%$ & NoFacility & NoFacility & $\begin{array}{l}\text { Major } \\
\text { Collector }\end{array}$ & 35 \\
\hline UniversitySouth24th & Summer & 68 & 32 & Thursday & $47 \%$ & $53 \%$ & NoFacility & NoFacility & $\langle N A\rangle$ & 65 \\
\hline GatewayStreetEast15 & Fall & 42 & 12 & Thursday & $29 \%$ & $71 \%$ & Lane & Lane & Minor Arterial & 44 \\
\hline
\end{tabular}


Table 3.21 - Scenario 2 AM Peak Factor Results NBPDP Factors

\begin{tabular}{|c|c|c|c|c|c|c|c|c|c|c|}
\hline \multicolumn{11}{|c|}{ NBPDP AM Factor } \\
\hline Location & Season & Observed & Estimate & Weekday & $\begin{array}{c}\% \\
\text { Difference }\end{array}$ & $\begin{array}{c}\text { Absolute } \\
\% \\
\text { Difference }\end{array}$ & $\begin{array}{c}\text { Bike } \\
\text { Facility }\end{array}$ & $\begin{array}{l}\text { Factor } \\
\text { Group }\end{array}$ & $\begin{array}{l}\text { Federal } \\
\text { Functional } \\
\text { Class }\end{array}$ & $\begin{array}{l}\text { Location } \\
\text { Id }\end{array}$ \\
\hline 11thWestAlder & Summer & 180 & 179 & Thursday & $99 \%$ & $1 \%$ & Lane & Lane & Minor Arterial & 72 \\
\hline 12thWestHigh & Fall & 466 & 462 & Tuesday & $99 \%$ & $1 \%$ & Blvd & Blvd & Local & 12 \\
\hline FirEastRiverRd & Fall & 319 & 315 & Thursday & $99 \%$ & $1 \%$ & NoFacility & NoFacility & Local & 19 \\
\hline GatewayStreetEast15 & Winter & 82 & 84 & Thursday & $102 \%$ & $2 \%$ & Lane & Lane & Minor Arterial & 44 \\
\hline PioneerPkwySouthQ & Winter & 123 & 126 & Tuesday & $102 \%$ & $2 \%$ & Path & Path-Rec & Path & 39 \\
\hline UniversitySouth18th & Summer & 203 & 200 & Thursday & $98 \%$ & $2 \%$ & NoFacility & NoFacility & Local & 71 \\
\hline PearlSouth19th & Fall & 119 & 116 & Thursday & $97 \%$ & $3 \%$ & Lane & Lane & $\begin{array}{l}\text { Major } \\
\text { Collector }\end{array}$ & 17 \\
\hline University South18th & Summer & 213 & 221 & Tuesday & $104 \%$ & $4 \%$ & NoFacility & NoFacility & Local & 71 \\
\hline HarrisSouth18th & Summer & 121 & 116 & Thursday & $95 \%$ & $5 \%$ & NoFacility & NoFacility & $\begin{array}{l}\text { Major } \\
\text { Collector }\end{array}$ & 73 \\
\hline MonroeSouth8th & Fall & 478 & 504 & Thursday & $105 \%$ & $5 \%$ & Blvd & Blvd & $\begin{array}{l}\text { Major } \\
\text { Collector }\end{array}$ & 11 \\
\hline NorthbankSouthGreenwayBr & Fall & 509 & 483 & Tuesday & $95 \%$ & $5 \%$ & Path & Path-Rec & Path & 3 \\
\hline 16thWestAlder & Summer & 167 & 158 & Thursday & $94 \%$ & $6 \%$ & NoFacility & NoFacility & Local & 70 \\
\hline SouthbankSouthGreenwayBr & Fall & 1098 & 1166 & Tuesday & $106 \%$ & $6 \%$ & Path & Path-Rec & Path & 4 \\
\hline NorthbankEastKnickerbocker & Fall & 596 & 641 & Thursday & $107 \%$ & $7 \%$ & Path & $\begin{array}{l}\text { Path- } \\
\text { Commute }\end{array}$ & Path & 97 \\
\hline 18thWestAlder & Fall & 646 & 588 & Thursday & $91 \%$ & $9 \%$ & Lane & Lane & Minor Arterial & 46 \\
\hline 15thEastAgate & Fall & 1021 & 1124 & Thursday & $110 \%$ & $10 \%$ & Blvd & Blvd & Local & 24 \\
\hline AgateSouth18th & Winter & 295 & 326 & Thursday & $110 \%$ & $10 \%$ & Lane & Lane & Minor Arterial & 25 \\
\hline 15thEastAgate & Winter & 814 & 903 & Tuesday & $111 \%$ & $11 \%$ & Blvd & Blvd & Local & 24 \\
\hline 42ndNorthCommercial & Winter & 189 & 210 & Thursday & $111 \%$ & $11 \%$ & Lane & Lane & Minor Arterial & 45 \\
\hline 15thWestJefferson & Winter & 348 & 305 & Thursday & $88 \%$ & $12 \%$ & Blvd & Blvd & Local & 29 \\
\hline 18thWestAlder & Summer & 352 & 305 & Thursday & $87 \%$ & $13 \%$ & Lane & Lane & Minor Arterial & 46 \\
\hline NorthbankWestDeltaHwy & Winter & 147 & 126 & Tuesday & $86 \%$ & $14 \%$ & Path & $\begin{array}{l}\text { Path- } \\
\text { Commute }\end{array}$ & Path & 16 \\
\hline UniversityNorth24th & Winter & 184 & 158 & Thursday & $86 \%$ & $14 \%$ & NoFacility & NoFacility & Local & 15 \\
\hline 12thEastLincoln & Summer & 513 & 588 & Thursday & $115 \%$ & $15 \%$ & Blvd & Blvd & Local & 28 \\
\hline FernRidgeWestChambers & Winter & 537 & 620 & Tuesday & $115 \%$ & $15 \%$ & Path & $\begin{array}{l}\text { Path- } \\
\text { Commute }\end{array}$ & Path & 95 \\
\hline FrohnmayerSouthRiver & Summer & 1084 & 1250 & Tuesday & $115 \%$ & $15 \%$ & Path & $\begin{array}{l}\text { Path- } \\
\text { Commute }\end{array}$ & Path & 6 \\
\hline PioneerPkwySouthQ & Fall & 190 & 158 & Tuesday & $83 \%$ & $17 \%$ & Path & Path-Rec & Path & 39 \\
\hline ThurstonEast58th & Winter & 36 & 42 & Thursday & $117 \%$ & $17 \%$ & Lane & Lane & Minor Arterial & 37 \\
\hline WillametteNorth15th & Fall & 330 & 273 & Tuesday & $83 \%$ & $17 \%$ & Lane & Lane & Minor Arterial & 13 \\
\hline 11thWestAlder & Summer & 209 & 168 & Tuesday & $80 \%$ & $20 \%$ & Lane & Lane & Minor Arterial & 72 \\
\hline 12thWesthigh & Winter & 393 & 315 & Tuesday & $80 \%$ & $20 \%$ & Blvd & Blvd & Local & 12 \\
\hline
\end{tabular}




\begin{tabular}{|c|c|c|c|c|c|c|c|c|c|c|}
\hline 18thWestAlder & Summer & 349 & 420 & Tuesday & $120 \%$ & $20 \%$ & Lane & Lane & Minor Arterial & 46 \\
\hline EastbankSouthOwossoBr & Fall & 488 & 389 & Thursday & $80 \%$ & $20 \%$ & Path & Path-Rec & Path & 2 \\
\hline NorthbankSouthGreenwayBr & Winter & 158 & 189 & Tuesday & $120 \%$ & $20 \%$ & Path & Path-Rec & Path & 3 \\
\hline 12thWestHigh & Summer & 372 & 294 & Thursday & $79 \%$ & $21 \%$ & Blvd & Blvd & Local & 12 \\
\hline DefazioSouthRiverEastBridge & Fall & 566 & 683 & Thursday & $121 \%$ & $21 \%$ & Path & $\begin{array}{l}\text { Path- } \\
\text { Commute }\end{array}$ & Path & 5 \\
\hline HighSouth4th & Winter & 331 & 399 & Thursday & $121 \%$ & $21 \%$ & NoFacility & NoFacility & $\begin{array}{l}\text { Major } \\
\text { Collector }\end{array}$ & 48 \\
\hline 12thWestHigh & Summer & 344 & 420 & Tuesday & $122 \%$ & $22 \%$ & Blvd & Blvd & Local & 12 \\
\hline DefazioSouthRiverEastBridge & Summer & 685 & 851 & Tuesday & $124 \%$ & $24 \%$ & Path & $\begin{array}{l}\text { Path- } \\
\text { Commute }\end{array}$ & Path & 5 \\
\hline 13thWestAlder & Fall & 2602 & 3255 & Tuesday & $125 \%$ & $25 \%$ & Lane & Lane & $\begin{array}{l}\text { Major } \\
\text { Collector }\end{array}$ & 21 \\
\hline GatewayStreetEastl5 & Fall & 42 & 53 & Thursday & $125 \%$ & $25 \%$ & Lane & Lane & Minor Arterial & 44 \\
\hline DEastPioneerPkwyPath & Fall & 75 & 95 & Thursday & $126 \%$ & $26 \%$ & NoFacility & NoFacility & Local & 38 \\
\hline DeltaBPBridgeEastGoodPasture & Fall & 207 & 263 & Thursday & $127 \%$ & $27 \%$ & Path & Path-Rec & Path & 31 \\
\hline AgateSouthFranklin & Fall & 563 & 725 & Tuesday & $129 \%$ & $29 \%$ & Lane & Lane & Minor Arterial & 23 \\
\hline FrohnmayerSouthRiver & Summer & 1094 & 1407 & Thursday & $129 \%$ & $29 \%$ & Path & $\begin{array}{l}\text { Path- } \\
\text { Commute }\end{array}$ & Path & 6 \\
\hline GWestMohawk & Fall & 112 & 147 & Tuesday & $131 \%$ & $31 \%$ & NoFacility & NoFacility & $\begin{array}{l}\text { Major } \\
\text { Collector }\end{array}$ & 35 \\
\hline 32ndSouthOregon & Fall & 878 & 599 & Thursday & $68 \%$ & $32 \%$ & Lane & Lane & Minor Arterial & 36 \\
\hline DefazioSouthRiverEastBridge & Summer & 650 & 861 & Thursday & $132 \%$ & $32 \%$ & Path & $\begin{array}{l}\text { Path- } \\
\text { Commute }\end{array}$ & Path & 5 \\
\hline GWestMohawk & Winter & 63 & 84 & Thursday & $133 \%$ & $33 \%$ & NoFacility & NoFacility & $\begin{array}{l}\text { Major } \\
\text { Collector }\end{array}$ & 35 \\
\hline 12thEastLincoln & Summer & 492 & 662 & Tuesday & $134 \%$ & $34 \%$ & Blvd & Blvd & Local & 28 \\
\hline GoodpastureEastDeltaHwy & Fall & 149 & 200 & Thursday & $134 \%$ & $34 \%$ & Lane & Lane & $\begin{array}{l}\text { Major } \\
\text { Collector }\end{array}$ & 1 \\
\hline UniversityNorth24th & Fall & 220 & 294 & Thursday & $134 \%$ & $34 \%$ & NoFacility & NoFacility & Local & 15 \\
\hline 16thWestAlder & Summer & 140 & 189 & Tuesday & $135 \%$ & $35 \%$ & NoFacility & NoFacility & Local & 70 \\
\hline 24thEastFilmore & Summer & 93 & 126 & Thursday & $135 \%$ & $35 \%$ & Lane & Lane & Minor Arterial & 88 \\
\hline 24thWestAdams & Summer & 117 & 158 & Thursday & $135 \%$ & $35 \%$ & Lane & Lane & $\begin{array}{l}\text { Major } \\
\text { Collector }\end{array}$ & 89 \\
\hline FernRidgeWestChambers & Winter & 497 & 672 & Thursday & $135 \%$ & $35 \%$ & Path & $\begin{array}{l}\text { Path- } \\
\text { Commute }\end{array}$ & Path & 95 \\
\hline MonroeSouth8th & Winter & 304 & 410 & Tuesday & $135 \%$ & $35 \%$ & Blvd & Blvd & $\begin{array}{l}\text { Major } \\
\text { Collector }\end{array}$ & 11 \\
\hline NorthbankWestDeltaHwy & Fall & 338 & 221 & Thursday & $65 \%$ & $35 \%$ & Path & $\begin{array}{l}\text { Path- } \\
\text { Commute }\end{array}$ & Path & 16 \\
\hline AgateSouth18th & Summer & 471 & 641 & Tuesday & $136 \%$ & $36 \%$ & Lane & Lane & Minor Arterial & 25 \\
\hline FrohnmayerSouthRiver & Fall & 1691 & 2321 & Tuesday & $137 \%$ & $37 \%$ & Path & $\begin{array}{l}\text { Path- } \\
\text { Commute }\end{array}$ & Path & 6 \\
\hline DeltaBPBridgeEastGoodPasture & Winter & 61 & 84 & Tuesday & $138 \%$ & $38 \%$ & Path & Path-Rec & Path & 31 \\
\hline EastbankSouthOwossoBr & Winter & 136 & 189 & Tuesday & $139 \%$ & $39 \%$ & Path & Path-Rec & Path & 2 \\
\hline HarlowEast15 & Winter & 434 & 609 & Thursday & $140 \%$ & $40 \%$ & Lane & Lane & Minor Arterial & 41 \\
\hline 15thWestAlder & Summer & 469 & 662 & Thursday & $141 \%$ & $41 \%$ & Blvd & Blvd & Local & 26 \\
\hline
\end{tabular}




\begin{tabular}{|c|c|c|c|c|c|c|c|c|c|c|}
\hline 15thWestAlder & Fall & 974 & 1376 & Tuesday & $141 \%$ & $41 \%$ & Blvd & Blvd & Local & 26 \\
\hline AgateSouthFranklin & Summer & 527 & 746 & Tuesday & $141 \%$ & $41 \%$ & Lane & Lane & Minor Arterial & 23 \\
\hline DEastPioneerPkwyPath & Winter & 74 & 105 & Tuesday & $142 \%$ & $42 \%$ & NoFacility & NoFacility & Local & 38 \\
\hline 13thWestAlder & Summer & 1392 & 1985 & Thursday & $143 \%$ & $43 \%$ & Lane & Lane & $\begin{array}{l}\text { Major } \\
\text { Collector }\end{array}$ & 21 \\
\hline OnyxNorthFranklin & Fall & 1121 & 1607 & Tuesday & $143 \%$ & $43 \%$ & NoFacility & NoFacility & $\begin{array}{l}\text { Major } \\
\text { Collector }\end{array}$ & 22 \\
\hline 18thWestAlder & Winter & 248 & 357 & Tuesday & $144 \%$ & $44 \%$ & Lane & Lane & Minor Arterial & 46 \\
\hline FirEastRiverRd & Winter & 175 & 252 & Thursday & $144 \%$ & $44 \%$ & NoFacility & NoFacility & Local & 19 \\
\hline HighSouth4th & Fall & 718 & 1040 & Thursday & $145 \%$ & $45 \%$ & NoFacility & NoFacility & $\begin{array}{l}\text { Major } \\
\text { Collector }\end{array}$ & 48 \\
\hline 15thWestJefferson & Fall & 798 & 1166 & Tuesday & $146 \%$ & $46 \%$ & Blvd & Blvd & Local & 29 \\
\hline 15thWestAlder & Winter & 579 & 851 & Tuesday & $147 \%$ & $47 \%$ & Blvd & Blvd & Local & 26 \\
\hline AgateSouthFranklin & Summer & 570 & 851 & Thursday & $149 \%$ & $49 \%$ & Lane & Lane & Minor Arterial & 23 \\
\hline GatewayBPBridgeEast15 & Winter & 120 & 179 & Thursday & $149 \%$ & $49 \%$ & Path & Path-Rec & Path & 40 \\
\hline PolkNorth24th & Summer & 105 & 158 & Thursday & $150 \%$ & $50 \%$ & NoFacility & NoFacility & Local & 90 \\
\hline UniversitySouth24th & Summer & 77 & 116 & Tuesday & $150 \%$ & $50 \%$ & NoFacility & NoFacility & $\begin{array}{l}\text { Major } \\
\text { Collector }\end{array}$ & 65 \\
\hline 13thWestAlder & Summer & 1335 & 2048 & Tuesday & $153 \%$ & $53 \%$ & Lane & Lane & $\begin{array}{l}\text { Major } \\
\text { Collector }\end{array}$ & 21 \\
\hline FernRidgeEastDanebo & Fall & 178 & 273 & Tuesday & $153 \%$ & $53 \%$ & Path & $\begin{array}{l}\text { Path- } \\
\text { Commute }\end{array}$ & Path & 9 \\
\hline HarlowEastl5 & Fall & 55 & 84 & Thursday & $153 \%$ & $53 \%$ & Lane & Lane & Minor Arterial & 41 \\
\hline AgateSouth18th & Summer & 438 & 683 & Thursday & $156 \%$ & $56 \%$ & Lane & Lane & Minor Arterial & 25 \\
\hline 13thWestAlder & Winter & 1631 & 2562 & Tuesday & $157 \%$ & $57 \%$ & Lane & Lane & $\begin{array}{l}\text { Major } \\
\text { Collector }\end{array}$ & 21 \\
\hline FrohnmayerSouthRiver & Winter & 637 & 998 & Thursday & $157 \%$ & $57 \%$ & Path & $\begin{array}{l}\text { Path- } \\
\text { Commute }\end{array}$ & Path & 6 \\
\hline RichardsonBridge & Fall & 427 & 672 & Tuesday & $157 \%$ & $57 \%$ & Path & $\begin{array}{l}\text { Path- } \\
\text { Commute }\end{array}$ & Path & 32 \\
\hline SouthbankSouthGreenwayBr & Winter & 576 & 914 & Thursday & $159 \%$ & $59 \%$ & Path & Path-Rec & Path & 4 \\
\hline WillametteNorth15th & Winter & 311 & 126 & Tuesday & $41 \%$ & $59 \%$ & Lane & Lane & Minor Arterial & 13 \\
\hline 12thEastLawrence & Winter & 495 & 798 & Tuesday & $161 \%$ & $61 \%$ & Blvd & Blvd & $\begin{array}{l}\text { Major } \\
\text { Collector }\end{array}$ & 96 \\
\hline 15thWestAlder & Summer & 477 & 767 & Tuesday & $161 \%$ & $61 \%$ & Blvd & Blvd & Local & 26 \\
\hline HeronBridgeSouthFernRidge & Fall & 372 & 599 & Tuesday & $161 \%$ & $61 \%$ & Path & $\begin{array}{l}\text { Path- } \\
\text { Commute }\end{array}$ & Path & 10 \\
\hline 24thWestAdams & Summer & 129 & 210 & Tuesday & $163 \%$ & $63 \%$ & Lane & Lane & $\begin{array}{l}\text { Major } \\
\text { Collector }\end{array}$ & 89 \\
\hline OnyxNorthFranklin & Summer & 658 & 1082 & Tuesday & $164 \%$ & $64 \%$ & NoFacility & NoFacility & $\begin{array}{l}\text { Major } \\
\text { Collector }\end{array}$ & 22 \\
\hline FriendlySouth18th & Fall & 217 & 357 & Tuesdav & $165 \%$ & $65 \%$ & Blvd & Blvd & $\begin{array}{l}\text { Major } \\
\text { Collector }\end{array}$ & 14 \\
\hline HeronBridgeSouthFernRidge & Winter & 342 & 567 & Tuesday & $166 \%$ & $66 \%$ & Path & $\begin{array}{l}\text { Path- } \\
\text { Commute }\end{array}$ & Path & 10 \\
\hline OnyxNorthFranklin & Summer & 702 & 1166 & Thursday & $166 \%$ & $66 \%$ & NoFacility & NoFacility & $\begin{array}{l}\text { Major } \\
\text { Collector }\end{array}$ & 22 \\
\hline
\end{tabular}




\begin{tabular}{|c|c|c|c|c|c|c|c|c|c|c|}
\hline DefazioSouthRiverEastBridge & Winter & 230 & 389 & Thursday & $169 \%$ & $69 \%$ & Path & $\begin{array}{l}\text { Path- } \\
\text { Commute }\end{array}$ & Path & 5 \\
\hline 15thEastAgate & Summer & 327 & 557 & Tuesday & $170 \%$ & $70 \%$ & Blvd & Blvd & Local & 24 \\
\hline FriendlySouth18th & Winter & 192 & 326 & Tuesday & $170 \%$ & $70 \%$ & Blvd & Blvd & $\begin{array}{l}\text { Major } \\
\text { Collector }\end{array}$ & 14 \\
\hline AlderNorth27th & Winter & 476 & 819 & Thursday & $172 \%$ & $72 \%$ & Blvd & Blvd & Local & 18 \\
\hline OnyxNorthFranklin & Winter & 707 & 1218 & Tuesday & $172 \%$ & $72 \%$ & NoFacility & NoFacility & $\begin{array}{l}\text { Major } \\
\text { Collector }\end{array}$ & 22 \\
\hline AlderSouthFranklin & Summer & 345 & 599 & Thursday & $173 \%$ & $73 \%$ & Blvd & Blvd & $\begin{array}{l}\text { Major } \\
\text { Collector }\end{array}$ & 27 \\
\hline AlderNorth27th & Fall & 702 & 1292 & Thursday & $184 \%$ & $84 \%$ & Blvd & Blvd & Local & 18 \\
\hline AlderSouthFranklin & Summer & 299 & 557 & Tuesday & $186 \%$ & $86 \%$ & Blvd & Blvd & $\begin{array}{l}\text { Major } \\
\text { Collector }\end{array}$ & 27 \\
\hline 15thEastAgate & Summer & 340 & 641 & Thursday & $188 \%$ & $88 \%$ & Blvd & Blvd & Local & 24 \\
\hline AgateSouthFranklin & Winter & 329 & 641 & Thursday & $195 \%$ & $95 \%$ & Lane & Lane & Minor Arterial & 23 \\
\hline HighNorth13th & Winter & 292 & 578 & Tuesday & $198 \%$ & $98 \%$ & Lane & Lane & Minor Arterial & 30 \\
\hline RichardsonBridge & Winter & 387 & 767 & Tuesday & $198 \%$ & $98 \%$ & Path & $\begin{array}{l}\text { Path- } \\
\text { Commute }\end{array}$ & Path & 32 \\
\hline AmazonPathNorth24th & Fall & 1023 & 2058 & Thursday & $201 \%$ & $101 \%$ & Path & $\begin{array}{l}\text { Path- } \\
\text { Commute }\end{array}$ & Path & 8 \\
\hline EWEBPathEastPioneerPkwy & Fall & 47 & 95 & Tuesday & $201 \%$ & $101 \%$ & Path & Path-Rec & Path & 33 \\
\hline PolkNorth24th & Summer & 88 & 179 & Tuesday & $203 \%$ & $103 \%$ & NoFacility & NoFacility & Local & 90 \\
\hline EWEBPathEast5th & Fall & 76 & 158 & Tuesday & $207 \%$ & $107 \%$ & Path & Path-Rec & Path & 34 \\
\hline 24thEastFilmore & Summer & 101 & 210 & Tuesday & $208 \%$ & $108 \%$ & Lane & Lane & Minor Arterial & 88 \\
\hline AlderSouthFranklin & Winter & 293 & 609 & Tuesday & $208 \%$ & $108 \%$ & Blvd & Blvd & $\begin{array}{l}\text { Major } \\
\text { Collector }\end{array}$ & 27 \\
\hline 13thEastHigh & Fall & 623 & 1302 & Thursday & $209 \%$ & $109 \%$ & Lane & Lane & $\begin{array}{l}\text { Major } \\
\text { Collector }\end{array}$ & 52 \\
\hline ClearwaterPath & Winter & 10 & 21 & Thursday & $210 \%$ & $110 \%$ & Path & Path-Rec & Path & 98 \\
\hline AmazonPathNorth24th & Winter & 538 & 1134 & Thursday & $211 \%$ & $111 \%$ & Path & $\begin{array}{l}\text { Path- } \\
\text { Commute }\end{array}$ & Path & 8 \\
\hline UniversitySouth24th & Summer & 68 & 147 & Thursday & $216 \%$ & $116 \%$ & NoFacility & NoFacility & $\begin{array}{l}\text { Major } \\
\text { Collector }\end{array}$ & 65 \\
\hline FernRidgeEastDanebo & Winter & 144 & 315 & Tuesday & $219 \%$ & $119 \%$ & Path & $\begin{array}{l}\text { Path- } \\
\text { Commute }\end{array}$ & Path & 9 \\
\hline 32ndSouthOregon & Winter & 36 & 84 & Thursday & $233 \%$ & $133 \%$ & Lane & Lane & Minor Arterial & 36 \\
\hline HighNorth13th & Fall & 299 & 704 & Tuesday & $235 \%$ & $135 \%$ & Lane & Lane & Minor Arterial & 30 \\
\hline GatewayBPBridgeEast15 & Fall & 95 & 231 & Thursday & $243 \%$ & $143 \%$ & Path & Path-Rec & Path & 40 \\
\hline DefazioNorthRiverWestBridge & Winter & 104 & 273 & Thursday & $262 \%$ & $162 \%$ & Path & $\begin{array}{l}\text { Path- } \\
\text { Commute }\end{array}$ & Path & 47 \\
\hline ThurstonEast58th & Fall & 44 & 158 & Thursday & $358 \%$ & $258 \%$ & Lane & Lane & Minor Arterial & 37 \\
\hline
\end{tabular}


Table 3.22 - Scenario 2 PM Peak Factor Results NBPDP Factors

\begin{tabular}{|c|c|c|c|c|c|c|c|c|c|c|}
\hline \multicolumn{11}{|c|}{ NBPDP PM Factor } \\
\hline Location & Season & Observed & Estimate & Weekday & $\begin{array}{c}\% \\
\text { Difference }\end{array}$ & $\begin{array}{c}\text { Absolute } \\
\% \\
\text { Difference }\end{array}$ & $\begin{array}{c}\text { Bike } \\
\text { Facility }\end{array}$ & $\begin{array}{l}\text { Factor } \\
\text { Group }\end{array}$ & $\begin{array}{c}\text { Federal } \\
\text { Functional } \\
\text { Class }\end{array}$ & $\begin{array}{l}\text { Location } \\
\text { Id }\end{array}$ \\
\hline AlderSouthFranklin & Winter & 293 & 294 & Tuesday & $100 \%$ & $0 \%$ & Blvd & Blvd & $\begin{array}{l}\text { Major } \\
\text { Collector } \\
\end{array}$ & 27 \\
\hline GWestMohawk & Fall & 112 & 112 & Tuesday & $100 \%$ & $0 \%$ & NoFacility & NoFacility & $\begin{array}{l}\text { Major } \\
\text { Collector }\end{array}$ & 35 \\
\hline PolkNorth24th & Summer & 105 & 105 & \begin{tabular}{|l} 
Thursday \\
\end{tabular} & $100 \%$ & $0 \%$ & \begin{tabular}{|l} 
NoFacility \\
\end{tabular} & NoFacility & Local & 90 \\
\hline 13thWestAlder & Summer & 1335 & 1323 & Tuesday & $99 \%$ & $1 \%$ & Lane & Lane & $\begin{array}{l}\text { Major } \\
\text { Collector }\end{array}$ & 21 \\
\hline 18thWestAlder & Summer & 352 & 350 & Thursday & $99 \%$ & $1 \%$ & Lane & Lane & Minor Arterial & 46 \\
\hline 18thWestAlder & Winter & 248 & 245 & Tuesday & $99 \%$ & $1 \%$ & Lane & Lane & Minor Arterial & 46 \\
\hline HarlowEast15 & Fall & 55 & 56 & Thursday & $101 \%$ & $1 \%$ & Lane & Lane & Minor Arterial & 41 \\
\hline 24thWestAdams & Summer & 129 & 126 & Tuesday & $98 \%$ & $2 \%$ & Lane & Lane & $\begin{array}{l}\text { Major } \\
\text { Collector }\end{array}$ & 89 \\
\hline GatewayStreetEast15 & Winter & 82 & 84 & \begin{tabular}{|l|} 
Thursday \\
\end{tabular} & $102 \%$ & $2 \%$ & Lane & Lane & Minor Arterial & 44 \\
\hline 13thWestAlder & Fall & 2602 & 2534 & Tuesday & $97 \%$ & $3 \%$ & Lane & Lane & $\begin{array}{l}\text { Major } \\
\text { Collector }\end{array}$ & 21 \\
\hline 15thEastAgate & Winter & 814 & 784 & Tuesday & $96 \%$ & $4 \%$ & Blvd & Blvd & Local & 24 \\
\hline 16thWestAlder & Summer & 167 & 161 & \begin{tabular}{|l} 
Thursday \\
\end{tabular} & $96 \%$ & $4 \%$ & NoFacility & NoFacility & Local & 70 \\
\hline 32ndSouthOregon & Winter & 36 & 35 & \begin{tabular}{|l} 
Thursday \\
\end{tabular} & $96 \%$ & $4 \%$ & \begin{tabular}{|l} 
Lane \\
\end{tabular} & Lane & Minor Arterial & 36 \\
\hline PolkNorth24th & Summer & 88 & 91 & Tuesday & $104 \%$ & $4 \%$ & \begin{tabular}{|l} 
NoFacility \\
\end{tabular} & NoFacility & Local & 90 \\
\hline WillametteNorth15th & Winter & 311 & 322 & Tuesday & $104 \%$ & $4 \%$ & \begin{tabular}{|l} 
Lane \\
\end{tabular} & Lane & Minor Arterial & 13 \\
\hline 16thWestAlder & Summer & 140 & 147 & Tuesday & $105 \%$ & $5 \%$ & \begin{tabular}{|l} 
NoFacility \\
\end{tabular} & NoFacility & Local & 70 \\
\hline HighNorth13th & Fall & 299 & 315 & Tuesday & $105 \%$ & $5 \%$ & Lane & Lane & Minor Arterial & 30 \\
\hline HighSouth4th & Winter & 331 & 308 & Thursday & $93 \%$ & $7 \%$ & NoFacility & NoFacility & $\begin{array}{l}\text { Major } \\
\text { Collector }\end{array}$ & 48 \\
\hline 12thEastLawrence & Winter & 495 & 546 & Tuesday & $110 \%$ & $10 \%$ & Blvd & Blvd & $\begin{array}{l}\text { Major } \\
\text { Collector }\end{array}$ & 96 \\
\hline 18thWestAlder & Summer & 349 & 385 & Tuesday & $110 \%$ & $10 \%$ & Lane & Lane & Minor Arterial & 46 \\
\hline 24thEastFilmore & Summer & 101 & 91 & Tuesday & $90 \%$ & $10 \%$ & Lane & Lane & Minor Arterial & 88 \\
\hline AlderSouthFranklin & Summer & 299 & 329 & Tuesday & $110 \%$ & $10 \%$ & Blvd & Blvd & $\begin{array}{l}\text { Major } \\
\text { Collector }\end{array}$ & 27 \\
\hline WillametteNorth15th & Fall & 330 & 364 & Tuesday & $110 \%$ & $10 \%$ & Lane & Lane & Minor Arterial & 13 \\
\hline 12thEastLincoln & Summer & 513 & 567 & Thursday & $111 \%$ & $11 \%$ & Blvd & Blvd & Local & 28 \\
\hline 13thWestAlder & Winter & 1631 & 1428 & Tuesday & $88 \%$ & $12 \%$ & Lane & Lane & $\begin{array}{l}\text { Major } \\
\text { Collector }\end{array}$ & 21 \\
\hline 15thEastAgate & Fall & 1021 & 896 & $\begin{array}{l}\text { Thursday } \\
\end{array}$ & $88 \%$ & $12 \%$ & Blvd & Blvd & Local & 24 \\
\hline 15thWestAlder & Summer & 477 & 546 & Tuesday & $114 \%$ & $14 \%$ & Blvd & Blvd & Local & 26 \\
\hline 13thWestAlder & Summer & 1392 & 1176 & Thursday & $84 \%$ & $16 \%$ & Lane & Lane & $\begin{array}{l}\text { Major } \\
\text { Collector }\end{array}$ & 21 \\
\hline AlderNorth27th & Winter & 476 & 553 & \begin{tabular}{|l} 
Thursday \\
\end{tabular} & $116 \%$ & $16 \%$ & Blvd & Blvd & Local & 18 \\
\hline
\end{tabular}




\begin{tabular}{|c|c|c|c|c|c|c|c|c|c|c|}
\hline HighSouth4th & Fall & 718 & 840 & Thursday & $117 \%$ & $17 \%$ & NoFacility & NoFacility & $\begin{array}{l}\text { Major } \\
\text { Collector }\end{array}$ & 48 \\
\hline OnyxNorthFranklin & Fall & 1121 & 1309 & Tuesday & $117 \%$ & $17 \%$ & NoFacility & NoFacility & $\begin{array}{l}\text { Major } \\
\text { Collector }\end{array}$ & 22 \\
\hline ThurstonEast58th & Winter & 36 & 42 & Thursday & $117 \%$ & $17 \%$ & Lane & Lane & Minor Arterial & 37 \\
\hline 12thEastLincoln & Summer & 492 & 581 & Tuesday & $118 \%$ & $18 \%$ & Blvd & Blvd & Local & 28 \\
\hline 18thWestAlder & Fall & 646 & 763 & Thursday & $118 \%$ & $18 \%$ & Lane & Lane & Minor Arterial & 46 \\
\hline GoodpastureEastDeltaHwy & Fall & 149 & 175 & Thursday & $118 \%$ & $18 \%$ & Lane & Lane & $\begin{array}{l}\text { Major } \\
\text { Collector } \\
\end{array}$ & \\
\hline 15thEastAgate & Summer & 327 & 392 & Tuesday & $120 \%$ & $20 \%$ & Blvd & Blvd & Local & 24 \\
\hline 15thWestAlder & Winter & 579 & 700 & Tuesday & $121 \%$ & $21 \%$ & Blvd & Blvd & Local & 26 \\
\hline FernRidgeWestChambers & Winter & 497 & 602 & Thursday & $121 \%$ & $21 \%$ & Path & $\begin{array}{l}\text { Path- } \\
\text { Commute }\end{array}$ & Path & 95 \\
\hline ThurstonEast58th & Fall & 44 & 35 & Thursday & $79 \%$ & $21 \%$ & Lane & Lane & Minor Arterial & 37 \\
\hline AlderSouthFranklin & Summer & 345 & 420 & Thursday & $122 \%$ & $22 \%$ & Blvd & Blvd & $\begin{array}{l}\text { Major } \\
\text { Collector }\end{array}$ & 27 \\
\hline OnyxNorthFranklin & Summer & 702 & 854 & Thursday & $122 \%$ & $22 \%$ & NoFacility & NoFacility & $\begin{array}{l}\text { Major } \\
\text { Collector }\end{array}$ & 22 \\
\hline 15thEastAgate & Summer & 340 & 420 & Thursday & $124 \%$ & $24 \%$ & Blvd & Blvd & Local & 24 \\
\hline 15thWestAlder & Fall & 974 & 1204 & Tuesday & $124 \%$ & $24 \%$ & Blvd & Blvd & Local & 26 \\
\hline AgateSouthFranklin & Winter & 329 & 406 & Thursday & $124 \%$ & $24 \%$ & Lane & Lane & Minor Arterial & 23 \\
\hline AmazonPathNorth24th & Winter & 538 & 665 & Thursday & $124 \%$ & $24 \%$ & Path & $\begin{array}{l}\text { Path- } \\
\text { Commute }\end{array}$ & Path & \\
\hline HarlowEast15 & Winter & 434 & 539 & Thursday & $124 \%$ & $24 \%$ & Lane & Lane & Minor Arterial & 41 \\
\hline PearlSouth19th & Fall & 119 & 147 & Thursday & $124 \%$ & $24 \%$ & Lane & Lane & $\begin{array}{l}\text { Major } \\
\text { Collector }\end{array}$ & 17 \\
\hline 13thEastHigh & Fall & 623 & 469 & Thursday & $75 \%$ & $25 \%$ & Lane & Lane & $\begin{array}{l}\text { Major } \\
\text { Collector }\end{array}$ & 52 \\
\hline 15thWestAlder & Summer & 469 & 588 & Thursday & $125 \%$ & $25 \%$ & Blvd & Blvd & Local & 26 \\
\hline DefazioSouthRiverEastBridge & Winter & 230 & 287 & Thursday & $125 \%$ & $25 \%$ & Path & $\begin{array}{l}\text { Path- } \\
\text { Commute }\end{array}$ & Path & 5 \\
\hline HeronBridgeSouthFernRidge & Winter & 342 & 427 & Tuesday & $125 \%$ & $25 \%$ & Path & \begin{tabular}{|l|} 
Path- \\
Commute
\end{tabular} & Path & 10 \\
\hline UniversitySouth18th & Summer & 213 & 266 & Tuesday & $125 \%$ & $25 \%$ & NoFacility & NoFacility & Local & 71 \\
\hline DefazioSouthRiverEastBridge & Summer & 650 & 819 & Thursday & $126 \%$ & $26 \%$ & Path & \begin{tabular}{|l|} 
Path- \\
Commute \\
\end{tabular} & Path & 5 \\
\hline UniversityNorth24th & Winter & 184 & 231 & Thursday & $126 \%$ & $26 \%$ & NoFacility & \begin{tabular}{|l} 
NoFacility \\
\end{tabular} & Local & 15 \\
\hline FrohnmayerSouthRiver & Winter & 637 & 812 & Thursday & $127 \%$ & $27 \%$ & Path & \begin{tabular}{|l|} 
Path- \\
Commute
\end{tabular} & Path & 6 \\
\hline 24thEastFilmore & Summer & 93 & 119 & Thursday & $128 \%$ & $28 \%$ & Lane & Lane & Minor Arterial & 88 \\
\hline EWEBPathEast5th & Fall & 76 & 98 & Tuesday & $128 \%$ & $28 \%$ & Path & Path-Rec & Path & 34 \\
\hline HarrisSouth18th & Summer & 121 & 154 & Thursday & $128 \%$ & $28 \%$ & NoFacility & NoFacility & $\begin{array}{l}\text { Major } \\
\text { Collector }\end{array}$ & 73 \\
\hline HighNorth13th & Winter & 292 & 210 & Tuesday & $72 \%$ & $28 \%$ & Lane & Lane & Minor Arterial & 30 \\
\hline 42ndNorthCommercial & Winter & 189 & 245 & Thursday & $129 \%$ & $29 \%$ & Lane & Lane & Minor Arterial & 45 \\
\hline AgateSouth18th & Summer & 438 & 567 & Thursday & $129 \%$ & $29 \%$ & Lane & Lane & Minor Arterial & 25 \\
\hline AgateSouthFranklin & Fall & 563 & 728 & Tuesday & $129 \%$ & $29 \%$ & Lane & Lane & Minor Arterial & 23 \\
\hline
\end{tabular}




\begin{tabular}{|c|c|c|c|c|c|c|c|c|c|c|}
\hline MonroeSouth8th & Fall & 478 & 616 & Thursday & $129 \%$ & $29 \%$ & Blvd & Blvd & $\begin{array}{l}\text { Major } \\
\text { Collector }\end{array}$ & 11 \\
\hline FirEastRiverRd & Fall & 319 & 420 & Thursday & $132 \%$ & $32 \%$ & NoFacility & NoFacility & Local & 19 \\
\hline GWestMohawk & Winter & 63 & 42 & Thursday & $67 \%$ & $33 \%$ & NoFacility & NoFacility & $\begin{array}{l}\text { Major } \\
\text { Collector }\end{array}$ & 35 \\
\hline SouthbankSouthGreenwayBr & Winter & 576 & 763 & Thursday & $133 \%$ & $33 \%$ & Path & Path-Rec & Path & 4 \\
\hline DefazioNorthRiverWestBridge & Winter & 104 & 140 & Thursday & $134 \%$ & $34 \%$ & Path & $\begin{array}{l}\text { Path- } \\
\text { Commute }\end{array}$ & Path & 47 \\
\hline FriendlySouth18th & Winter & 192 & 259 & Tuesday & $135 \%$ & $35 \%$ & Blvd & Blvd & $\begin{array}{l}\text { Major } \\
\text { Collector }\end{array}$ & 14 \\
\hline FirEastRiverRd & Winter & 175 & 238 & Thursday & $136 \%$ & $36 \%$ & NoFacility & \begin{tabular}{|l} 
NoFacility \\
\end{tabular} & Local & 19 \\
\hline OnyxNorthFranklin & Summer & 658 & 896 & Tuesday & $136 \%$ & $36 \%$ & NoFacility & NoFacility & $\begin{array}{l}\text { Major } \\
\text { Collector }\end{array}$ & 22 \\
\hline 11thWestAlder & Summer & 209 & 287 & Tuesday & $137 \%$ & $37 \%$ & Lane & Lane & Minor Arterial & 72 \\
\hline PioneerPkwySouthQ & Fall & 190 & 259 & Tuesday & $137 \%$ & $37 \%$ & Path & Path-Rec & Path & 39 \\
\hline AgateSouthFranklin & Summer & 527 & 728 & Tuesday & $138 \%$ & $38 \%$ & Lane & Lane & Minor Arterial & 23 \\
\hline NorthbankWestDeltaHwy & Winter & 147 & 203 & Tuesday & $138 \%$ & $38 \%$ & Path & $\begin{array}{l}\text { Path- } \\
\text { Commute }\end{array}$ & Path & 16 \\
\hline UniversitySouth24th & Summer & 68 & 42 & Thursday & $62 \%$ & $38 \%$ & NoFacility & NoFacility & $\begin{array}{l}\text { Major } \\
\text { Collector }\end{array}$ & 65 \\
\hline UniversitySouth18th & Summer & 203 & 287 & Thursday & $141 \%$ & $41 \%$ & NoFacility & NoFacility & Local & 71 \\
\hline FrohnmayerSouthRiver & Summer & 1084 & 1540 & Tuesday & $142 \%$ & $42 \%$ & Path & \begin{tabular}{|l} 
Path- \\
Commute
\end{tabular} & Path & 6 \\
\hline MonroeSouth8th & Winter & 304 & 434 & Tuesday & $143 \%$ & $43 \%$ & Blvd & Blvd & $\begin{array}{l}\text { Major } \\
\text { Collector }\end{array}$ & 11 \\
\hline PioneerPkwySouthQ & Winter & 123 & 175 & Tuesday & $143 \%$ & $43 \%$ & Path & Path-Rec & Path & 39 \\
\hline 12thWestHigh & Summer & 344 & 497 & Tuesday & $144 \%$ & $44 \%$ & Blvd & Blvd & Local & 12 \\
\hline 24thWestAdams & Summer & 117 & 168 & Thursday & $144 \%$ & $44 \%$ & Lane & Lane & $\begin{array}{l}\text { Major } \\
\text { Collector }\end{array}$ & 89 \\
\hline OnyxNorthFranklin & Winter & 707 & 1022 & Tuesday & $145 \%$ & $45 \%$ & NoFacility & NoFacility & $\begin{array}{l}\text { Major } \\
\text { Collector }\end{array}$ & 22 \\
\hline AgateSouthFranklin & Summer & 570 & 833 & Thursday & $146 \%$ & $46 \%$ & Lane & Lane & Minor Arterial & 23 \\
\hline FernRidgeWestChambers & Winter & 537 & 791 & Tuesday & $147 \%$ & $47 \%$ & Path & $\begin{array}{l}\text { Path- } \\
\text { Commute }\end{array}$ & Path & 95 \\
\hline FrohnmayerSouthRiver & Fall & 1691 & 2478 & Tuesday & $147 \%$ & $47 \%$ & Path & \begin{tabular}{|l|} 
Path- \\
Commute
\end{tabular} & Path & 6 \\
\hline UniversityNorth24th & Fall & 220 & 322 & Thursday & $147 \%$ & $47 \%$ & NoFacility & NoFacility & Local & 15 \\
\hline AlderNorth27th & Fall & 702 & 1036 & Thursday & $148 \%$ & $48 \%$ & Blvd & Blvd & Local & 18 \\
\hline 12thWestHigh & Fall & 466 & 693 & Tuesday & $149 \%$ & $49 \%$ & Blvd & Blvd & Local & 12 \\
\hline 12thWestHigh & Winter & 393 & 588 & Tuesday & $150 \%$ & $50 \%$ & Blvd & Blvd & Local & 12 \\
\hline AmazonPathNorth24th & Fall & 1023 & 1533 & Thursday & $150 \%$ & $50 \%$ & Path & $\begin{array}{l}\text { Path- } \\
\text { Commute }\end{array}$ & Path & 8 \\
\hline DeltaBPBridgeEastGoodPasture & Winter & 61 & 91 & Tuesday & $150 \%$ & $50 \%$ & Path & Path-Rec & Path & 31 \\
\hline 11thWestAlder & Summer & 180 & 273 & Thursday & $152 \%$ & $52 \%$ & Lane & Lane & Minor Arterial & 72 \\
\hline 15thWestJefferson & Winter & 348 & 539 & Thursday & $155 \%$ & $55 \%$ & Blvd & Blvd & Local & 29 \\
\hline DefazioSouthRiverEastBridge & Fall & 566 & 875 & Thursday & $155 \%$ & $55 \%$ & Path & $\begin{array}{l}\text { Path- } \\
\text { Commute }\end{array}$ & Path & \\
\hline
\end{tabular}




\begin{tabular}{|c|c|c|c|c|c|c|c|c|c|c|}
\hline GatewayBPBridgeEast15 & Winter & 120 & 189 & Thursday & $157 \%$ & $57 \%$ & Path & Path-Rec & Path & 40 \\
\hline AgateSouth18th & Summer & 471 & 742 & Tuesday & $158 \%$ & $58 \%$ & Lane & Lane & Minor Arterial & 25 \\
\hline DefazioSouthRiverEastBridge & Summer & 685 & 1092 & Tuesday & $159 \%$ & $59 \%$ & Path & $\begin{array}{l}\text { Path- } \\
\text { Commute }\end{array}$ & Path & 5 \\
\hline DEastPioneerPkwyPath & Winter & 74 & 119 & Tuesday & $160 \%$ & $60 \%$ & NoFacility & NoFacility & Local & 38 \\
\hline FrohnmayerSouthRiver & Summer & 1094 & 1750 & Thursday & $160 \%$ & $60 \%$ & Path & $\begin{array}{l}\text { Path- } \\
\text { Commute }\end{array}$ & Path & 6 \\
\hline HeronBridgeSouthFernRidge & Fall & 372 & 595 & Tuesday & $160 \%$ & $60 \%$ & Path & $\begin{array}{l}\text { Path- } \\
\text { Commute }\end{array}$ & Path & 10 \\
\hline AgateSouth18th & Winter & 295 & 476 & Thursday & $161 \%$ & $61 \%$ & Lane & Lane & Minor Arterial & 25 \\
\hline 12thWestHigh & Summer & 372 & 602 & Thursday & $162 \%$ & $62 \%$ & Blvd & Blvd & Local & 12 \\
\hline 15thWestJefferson & Fall & 798 & 1302 & Tuesday & $163 \%$ & $63 \%$ & Blvd & Blvd & Local & 29 \\
\hline NorthbankEastKnickerbocker & Fall & 596 & 973 & Thursday & $163 \%$ & $63 \%$ & Path & $\begin{array}{l}\text { Path- } \\
\text { Commute }\end{array}$ & Path & 97 \\
\hline NorthbankWestDeltaHwy & Fall & 338 & 553 & Thursday & $164 \%$ & $64 \%$ & Path & $\begin{array}{l}\begin{array}{l}\text { Path- } \\
\text { Commute }\end{array} \\
\end{array}$ & Path & 16 \\
\hline FriendlySouth18th & Fall & 217 & 357 & Tuesday & $165 \%$ & $65 \%$ & Blvd & Blvd & $\begin{array}{l}\text { Major } \\
\text { Collector }\end{array}$ & 14 \\
\hline RichardsonBridge & Fall & 427 & 707 & Tuesday & $165 \%$ & $65 \%$ & Path & $\begin{array}{l}\begin{array}{l}\text { Path- } \\
\text { Commute }\end{array} \\
\end{array}$ & Path & 32 \\
\hline GatewayStreetEast15 & Fall & 42 & 14 & Thursday & $32 \%$ & $68 \%$ & Lane & Lane & Minor Arterial & 44 \\
\hline FernRidgeEastDanebo & Fall & 178 & 301 & Tuesday & $169 \%$ & $69 \%$ & Path & $\begin{array}{l}\begin{array}{l}\text { Path- } \\
\text { Commute }\end{array} \\
\end{array}$ & Path & 9 \\
\hline EastbankSouthOwossoBr & Winter & 136 & 231 & Tuesday & $170 \%$ & $70 \%$ & Path & Path-Rec & Path & 2 \\
\hline 32ndSouthOregon & Fall & 878 & 1512 & Thursday & $172 \%$ & $72 \%$ & Lane & Lane & Minor Arterial & 36 \\
\hline RichardsonBridge & Winter & 387 & 672 & Tuesday & $174 \%$ & $74 \%$ & Path & $\begin{array}{l}\text { Path- } \\
\text { Commute }\end{array}$ & Path & 32 \\
\hline DEastPioneerPkwyPath & Fall & 75 & 133 & Thursday & $178 \%$ & $78 \%$ & NoFacility & NoFacility & Local & 38 \\
\hline EWEBPathEastPioneerPkwy & Fall & 47 & 84 & Tuesday & $179 \%$ & $79 \%$ & Path & Path-Rec & Path & 33 \\
\hline SouthbankSouthGreenwayBr & Fall & 1098 & 1981 & Tuesday & $180 \%$ & $80 \%$ & Path & Path-Rec & Path & 4 \\
\hline NorthbankSouthGreenwayBr & Winter & 158 & 294 & Tuesday & $186 \%$ & $86 \%$ & Path & Path-Rec & Path & 3 \\
\hline UniversitySouth24th & Summer & 77 & 147 & Tuesday & $191 \%$ & $91 \%$ & NoFacility & NoFacility & $\begin{array}{l}\text { Major } \\
\text { Collector }\end{array}$ & 65 \\
\hline GatewayBPBridgeEast15 & Fall & 95 & 189 & Thursday & $199 \%$ & $99 \%$ & Path & Path-Rec & Path & 40 \\
\hline EastbankSouthOwossoBr & Fall & 488 & 994 & Thursday & $204 \%$ & $104 \%$ & Path & Path-Rec & Path & 2 \\
\hline FernRidgeEastDanebo & Winter & 144 & 301 & Tuesday & $209 \%$ & $109 \%$ & Path & $\begin{array}{l}\text { Path- } \\
\text { Commute }\end{array}$ & Path & 9 \\
\hline ClearwaterPath & Winter & 10 & 21 & Thursday & $210 \%$ & $110 \%$ & Path & Path-Rec & Path & 98 \\
\hline NorthbankSouthGreenwayBr & Fall & 509 & 1071 & Tuesday & $210 \%$ & $110 \%$ & Path & Path-Rec & Path & 3 \\
\hline DeltaBPBridgeEastGoodPasture & Fall & 207 & 483 & Thursday & $233 \%$ & $133 \%$ & Path & Path-Rec & Path & 31 \\
\hline ThurstonEast58th & & 44 & 158 & Thursday & $358 \%$ & $258 \%$ & Lane & Lane & Minor Arterial & 37 \\
\hline
\end{tabular}


These results indicate that error in estimating total daily bicycle traffic from 2-hour time-of-day factors can be reduced with more refined factors. Figure 3.16 demonstrates graphically the results presented in the tables above. The results using time-of-day factors based on study area data are featured in the top two plots with the results from the NBPDP factors application are presented in the bottom two plots. Each of these factors' results are presented using both an AM and PM period sample and respective factor. This plot also summarizes the R-squared and RMSE the average absolute percentage difference while also describing the mean absolute percent difference by factor group.

\section{Figure 3.16 - Results for Scenario 2 Study Area Factors Compared to NBPDP Factors}

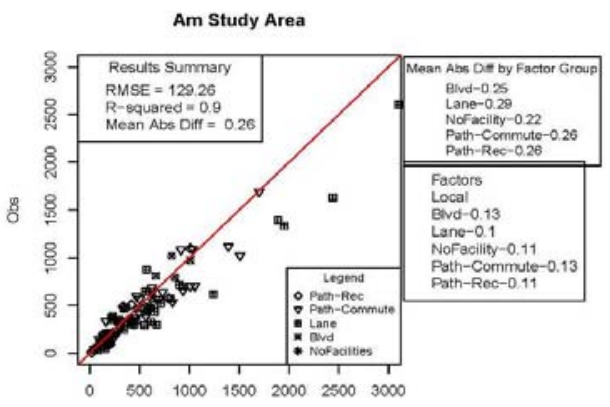

Est

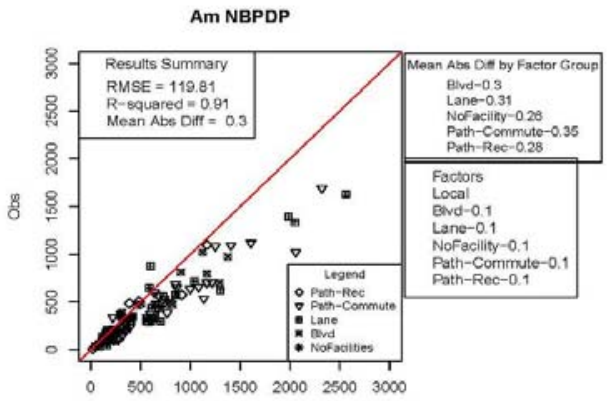

Est

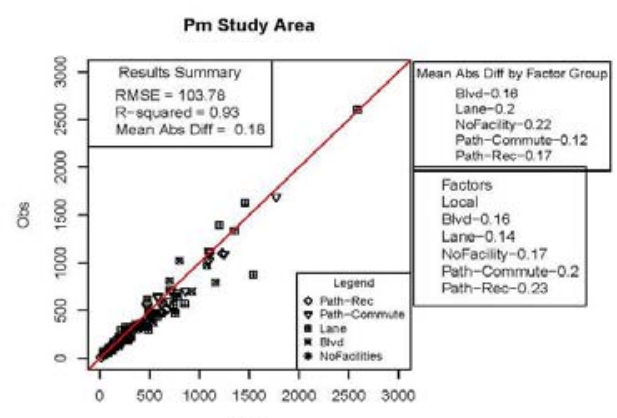

Est

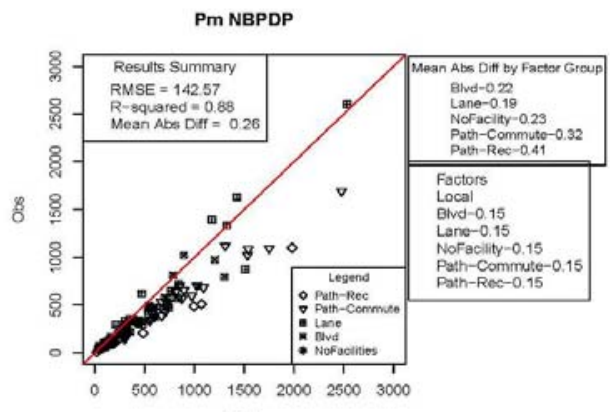

Est 
Points in the plots in Figure 3.16 that appear above the line indicate an under estimate while points on the plot below the red line indicate an over estimation. Table 3.23 sums all of the estimates for each factor application and compares with the sum of observed counts to better understand the relative levels of over versus under estimation. Each of the factor application s result in an over estimate but the study area PM factors appear to over estimate by the least amount when reviewed in this way.

Table 3.23- Relative Levels of Over-estimation

\begin{tabular}{|c|c|c|c|}
\hline Factors & $\begin{array}{c}\text { Sum of All } \\
\text { Estimates }\end{array}$ & Observed & $\begin{array}{c}\text { Percent } \\
\text { Difference }\end{array}$ \\
\hline $\begin{array}{c}\text { Study Area } \\
\text { AM }\end{array}$ & 56,214 & 48,617 & $116 \%$ \\
\hline $\begin{array}{c}\text { Study Area } \\
\text { PM }\end{array}$ & 53,608 & 48,617 & $110 \%$ \\
\hline NBPDP AM & 67,777 & 48,617 & $139 \%$ \\
\hline NBPDP PM & 63,474 & 48,617 & $131 \%$ \\
\hline
\end{tabular}

Table 3.24 reviews the results by removing count locations that could be considered outliers because of low overall daily traffic. Locations with less than 100 daily cyclists could have substantial variation in hourly traffic which could result in daily estimates with much more error. Removing these locations does improve the average absolute percentage difference for all factor implementations.however slightly. 


\section{Table 3.24- Exploring Effect of Outliers- Average Absolute \% Difference Results Comparison}

\begin{tabular}{|c|c|c|c|c|}
\hline & $\begin{array}{c}\text { Average } \\
\text { Absolute \% } \\
\text { Difference for } \\
\text { locations less } \\
\text { than 100 Daily } \\
\text { Bicyclists }\end{array}$ & $\begin{array}{c}\text { Average } \\
\text { Absolute \% } \\
\text { Difference }\end{array}$ & $\begin{array}{c}\text { Average Absolute } \\
\text { locations more } \\
\text { than 100 Daily } \\
\text { Bicyclists }\end{array}$ & Period \\
\hline Study Area & $24 \%$ & $31 \%$ & $23 \%$ & AM \\
\hline Study Area & $16 \%$ & $26 \%$ & $14 \%$ & PM \\
\hline NBPDP & $48 \%$ & $77 \%$ & $43 \%$ & AM \\
\hline NBPDP & $37 \%$ & $45 \%$ & $36 \%$ & PM \\
\hline Observations & 123 & 18 & 105 & \\
\hline
\end{tabular}

Detailed in Table 3.23 below is the average absolute percentage difference by factor group type.

Table 3.25- Average Absolute Percentage Difference by Factor Group

\begin{tabular}{|c|c|c|c|c|c|c|}
\hline $\begin{array}{c}\text { Factors } \\
\text { Study Area } \\
\text { AM }\end{array}$ & Blvd & Lane & No Facility & $\begin{array}{c}\text { Path- } \\
\text { Commute }\end{array}$ & $\begin{array}{c}\text { Path- } \\
\text { Recreation }\end{array}$ & Average \\
\hline $\begin{array}{c}\text { Study Area } \\
\text { PM }\end{array}$ & $20 \%$ & $28 \%$ & $18 \%$ & $26 \%$ & $28 \%$ & $24 \%$ \\
\hline NBPDP AM & $46 \%$ & $19 \%$ & $19 \%$ & $11 \%$ & $16 \%$ & $16 \%$ \\
\hline NBPDP PM & $30 \%$ & $50 \%$ & $39 \%$ & $55 \%$ & $50 \%$ & $48 \%$ \\
\hline
\end{tabular}

Additional breakdowns of the Scenario 2 results are presented in tables below. Table 3.26 presents the results broken out by the season in which the counts were collected to examine any differences in performance of the estimation. For the AM study area 
factors result in estimates with less error for the summer results while the study area PM results are identical across seasons. The summer results have slightly less error for both the AM and PM NBPDP factors compared to the average.

Table 3.26 - Average Absolute Percentage Difference by Season

\begin{tabular}{|c|c|c|c|c|}
\hline \multicolumn{5}{|c|}{ Average Absolute Percentage Difference by Season } \\
\hline Factors & Summer & Fall & Winter & Average \\
\hline Study Area AM & $19 \%$ & $28 \%$ & $25 \%$ & $24 \%$ \\
\hline Study Area PM & $16 \%$ & $16 \%$ & $16 \%$ & $16 \%$ \\
\hline NBPDP AM & $43 \%$ & $48 \%$ & $53 \%$ & $48 \%$ \\
\hline NBPDP PM & $28 \%$ & $47 \%$ & $35 \%$ & $37 \%$ \\
\hline Observations & 39 & 41 & 43 & 123 \\
\hline
\end{tabular}

Since some of the factors were applied to data collected on Tuesday and some of

Thursday a breakdown of the error is presented in Table 3.27. For the results using study area factors there are slight differences of $2 \%$ and $5 \%$ between the Tuesday and Thursday results for the AM and PM factors respectively while there is no real difference between the Tuesday and Thursday results for either period for the NBPDP factors.

Table 3.27 - Average Absolute Percentage Difference by Tuesday/Thursday

\begin{tabular}{|l|c|c|}
\hline \multicolumn{3}{|c|}{$\begin{array}{c}\text { Average Absolute Percentage } \\
\text { Difference by Tuesday/Thursday }\end{array}$} \\
\hline Factors & Thursday & Tuesday \\
\hline $\begin{array}{l}\text { Study Area } \\
\text { AM }\end{array}$ & $27 \%$ & $22 \%$ \\
\hline $\begin{array}{l}\text { Study Area } \\
\text { PM }\end{array}$ & $17 \%$ & $15 \%$ \\
\hline NBPDP AM & $48 \%$ & $49 \%$ \\
\hline NBPDP PM & $37 \%$ & $37 \%$ \\
\hline Observations & 63 & 60 \\
\hline
\end{tabular}


The above tables and plots summarize the results from the application of the Scenario 2 factors derived from study area data and compared with NBPDP factors. A review of these results, as well as the results from the Scenario 1 application will follow in the next section, including discussion on how the factors tested above compare with factors from other data sources including the NHTS and OHAS surveys. 


\subsection{3 - Comparison of Scenario 1 and Scenario 2 Factor Results}

To further the understanding of how well the factors from Scenario 1 and 2 compare, an evaluation of the estimates for the 32 count locations analyzed in Section 3.5.1 (and detailed in Figure 3.14 above) will be presented. Tables 3.26 through 3.29 detail the comparisons of estimates and their measures of absolute percentage difference. The Scenario 2 results presented above were done for 123 locations available for application, including the 32 previously tested in the Scenario 1 application of factors. 
Table 3.28 - Comparison of Scenario 1 and Scenario 2 AM Study Area Factors

\begin{tabular}{|c|c|c|c|c|c|}
\hline \multicolumn{6}{|c|}{ Comparison of Scenario 1 and Scenario 2 AM Study Area Factors } \\
\hline Location & Observed & $\begin{array}{l}\text { Scenario } \\
1 \\
\text { Estimate }\end{array}$ & $\begin{array}{l}\text { Scenario } \\
2 \\
\text { Estimate }\end{array}$ & $\begin{array}{l}\text { Scenario } 1 \\
\text { Absolute \% } \\
\text { Difference }\end{array}$ & $\begin{array}{l}\text { Scenario } \\
2 \\
\text { Absolute } \\
\% \\
\text { Difference }\end{array}$ \\
\hline 15thWestJefferson & 798 & 867 & 740 & $9 \%$ & $7 \%$ \\
\hline AlderNorth27th & 702 & 961 & 820 & $37 \%$ & $17 \%$ \\
\hline AmazonPathNorth24th & 1023 & 1782 & 1508 & $74 \%$ & $47 \%$ \\
\hline DEastPioneerPkwyPath & 75 & 69 & 69 & $8 \%$ & $8 \%$ \\
\hline DefazioSouthRiverEastBridge & 566 & 591 & 500 & $4 \%$ & $12 \%$ \\
\hline DeltaBPBridgeEastGoodPasture & 207 & 227 & 167 & $10 \%$ & $19 \%$ \\
\hline EastbankSouthOwossoBr & 488 & 336 & 247 & $31 \%$ & $49 \%$ \\
\hline EWEBPathEast5th & 76 & 136 & 100 & $79 \%$ & $32 \%$ \\
\hline EWEBPathEastPioneerPkwy & 47 & 82 & 60 & $74 \%$ & $28 \%$ \\
\hline FernRidgeEastDanebo & 178 & 236 & 200 & $33 \%$ & $12 \%$ \\
\hline FirEastRiverRd & 319 & 231 & 231 & $28 \%$ & $28 \%$ \\
\hline FriendlySouth18th & 217 & 266 & 227 & $23 \%$ & $5 \%$ \\
\hline GatewayBPBridgeEastl5 & 95 & 200 & 147 & $111 \%$ & $55 \%$ \\
\hline GatewayStreetEastl5 & 42 & 39 & 33 & $7 \%$ & $21 \%$ \\
\hline GoodpastureEastDeltaHwy & 149 & 148 & 127 & $1 \%$ & $15 \%$ \\
\hline GWestMohawk & 112 & 108 & 108 & $4 \%$ & $4 \%$ \\
\hline HarlowEastl5 & 55 & 62 & 53 & $13 \%$ & $4 \%$ \\
\hline HeronBridgeSouthFernRidge & 372 & 518 & 438 & $39 \%$ & $18 \%$ \\
\hline HighNorth13th & 299 & 523 & 447 & $75 \%$ & $49 \%$ \\
\hline HighSouth4th & 718 & 762 & 762 & $6 \%$ & $6 \%$ \\
\hline MonroeSouth8th & 478 & 375 & 320 & $22 \%$ & $33 \%$ \\
\hline
\end{tabular}




\begin{tabular}{|c|c|c|c|c|c|}
\hline NorthbankEastKnickerbocker & 596 & 555 & 469 & $7 \%$ & $21 \%$ \\
\hline NorthbankSouthGreenwayBr & 509 & 418 & 307 & $18 \%$ & $40 \%$ \\
\hline NorthbankWestDeltaHwy & 338 & 191 & 162 & $43 \%$ & $52 \%$ \\
\hline PearlSouth19th & 119 & 86 & 73 & $28 \%$ & $39 \%$ \\
\hline PioneerPkwySouthQ & 190 & 136 & 100 & $28 \%$ & $47 \%$ \\
\hline RichardsonBridge & 427 & 582 & 492 & $36 \%$ & $15 \%$ \\
\hline SouthbankSouthGreenwayBr & 1098 & 1009 & 740 & $8 \%$ & $33 \%$ \\
\hline ThurstonEast58th & 44 & 117 & 100 & $166 \%$ & $127 \%$ \\
\hline UniversityNorth24th & 220 & 215 & 215 & $2 \%$ & $2 \%$ \\
\hline WillametteNorth15th & 330 & 203 & 173 & $38 \%$ & $48 \%$ \\
\hline
\end{tabular}


Table 3.29 - Comparison of Scenario 1 and Scenario 2 PM Study Area Factors

\begin{tabular}{|c|c|c|c|c|c|}
\hline \multicolumn{6}{|c|}{ Comparison of Scenario 1 and Scenario 2 PM Study Area Factors } \\
\hline Location & Observed & $\begin{array}{l}\text { Scenario } \\
1 \\
\text { Estimate }\end{array}$ & $\begin{array}{l}\text { Scenario } \\
2 \\
\text { Estimate }\end{array}$ & $\begin{array}{l}\text { Scenario } 1 \\
\text { Absolute \% } \\
\text { Difference }\end{array}$ & $\begin{array}{l}\text { Scenario } \\
2 \\
\text { Absolute } \\
\% \\
\text { Difference } \\
\end{array}$ \\
\hline 15thWestJefferson & 798 & 1022 & 979 & $28 \%$ & $23 \%$ \\
\hline AlderNorth27th & 702 & 813 & 779 & $16 \%$ & $11 \%$ \\
\hline AmazonPathNorth24th & 1023 & 1043 & 1043 & $2 \%$ & $2 \%$ \\
\hline DEastPioneerPkwyPath & 75 & 113 & 100 & $51 \%$ & $33 \%$ \\
\hline DefazioSouthRiverEastBridge & 566 & 595 & 595 & $5 \%$ & $5 \%$ \\
\hline DeltaBPBridgeEastGoodPasture & 207 & 329 & 276 & $59 \%$ & $33 \%$ \\
\hline EastbankSouthOwossoBr & 488 & 676 & 568 & $39 \%$ & $16 \%$ \\
\hline EWEBPathEast5th & 76 & 67 & 56 & $12 \%$ & $26 \%$ \\
\hline EWEBPathEastPioneerPkwy & 47 & 57 & 48 & $21 \%$ & $2 \%$ \\
\hline FernRidgeEastDanebo & 178 & 205 & 205 & $15 \%$ & $15 \%$ \\
\hline FirEastRiverRd & 319 & 356 & 316 & $12 \%$ & $1 \%$ \\
\hline FriendlySouth18th & 217 & 280 & 268 & $29 \%$ & $24 \%$ \\
\hline GatewayBPBridgeEastl5 & 95 & 129 & 108 & $36 \%$ & $14 \%$ \\
\hline GatewayStreetEastl5 & 42 & 12 & 12 & $71 \%$ & $71 \%$ \\
\hline GoodpastureEastDeltaHwy & 149 & 154 & 147 & $3 \%$ & $1 \%$ \\
\hline GWestMohawk & 112 & 95 & 84 & $15 \%$ & $25 \%$ \\
\hline HarlowEastl5 & 55 & 49 & 47 & $11 \%$ & $15 \%$ \\
\hline HeronBridgeSouthFernRidge & 372 & 405 & 405 & $9 \%$ & $9 \%$ \\
\hline
\end{tabular}




\begin{tabular}{|c|c|c|c|c|c|}
\hline HighNorth13th & 299 & 278 & 265 & $7 \%$ & $11 \%$ \\
\hline HighSouth4th & 718 & 713 & 632 & $1 \%$ & $12 \%$ \\
\hline MonroeSouth8th & 478 & 484 & 463 & $1 \%$ & $3 \%$ \\
\hline NorthbankEastKnickerbocker & 596 & 662 & 662 & $11 \%$ & $11 \%$ \\
\hline NorthbankSouthGreenwayBr & 509 & 729 & 612 & $43 \%$ & $20 \%$ \\
\hline NorthbankWestDeltaHwy & 338 & 376 & 376 & $11 \%$ & $11 \%$ \\
\hline PearlSouth19th & 119 & 130 & 124 & $9 \%$ & $4 \%$ \\
\hline PioneerPkwySouthQ & 190 & 176 & 148 & $7 \%$ & $22 \%$ \\
\hline RichardsonBridge & 427 & 481 & 481 & $13 \%$ & $13 \%$ \\
\hline SouthbankSouthGreenwayBr & 1098 & 1348 & 1132 & $23 \%$ & $3 \%$ \\
\hline ThurstonEast58th & 44 & 31 & 29 & $30 \%$ & $34 \%$ \\
\hline UniversityNorth24th & 220 & 273 & 242 & $24 \%$ & $10 \%$ \\
\hline WillametteNorth15th & 330 & 321 & 306 & $3 \%$ & $7 \%$ \\
\hline
\end{tabular}


Table 3.30 - Comparison of Scenario 1 and Scenario 2 AM NBPDP Factors

\begin{tabular}{|c|c|c|c|c|c|}
\hline \multicolumn{6}{|c|}{ Comparison of Scenario 1 and Scenario 2 AM NBPDP Factors } \\
\hline Location & Observed & $\begin{array}{l}\text { Scenario } \\
1 \\
\text { Estimate }\end{array}$ & $\begin{array}{l}\text { Scenario } \\
2 \\
\text { Estimate }\end{array}$ & $\begin{array}{l}\text { Scenario } 1 \\
\text { Absolute \% } \\
\text { Difference }\end{array}$ & $\begin{array}{l}\text { Scenario } \\
2 \\
\text { Absolute } \\
\% \\
\text { Difference }\end{array}$ \\
\hline 15thWestJefferson & 798 & 1166 & 1166 & $46 \%$ & $46 \%$ \\
\hline AlderNorth27th & 702 & 1292 & 1292 & $84 \%$ & $84 \%$ \\
\hline AmazonPathNorth24th & 1023 & 2058 & 2058 & $101 \%$ & $101 \%$ \\
\hline DEastPioneerPkwyPath & 75 & 94 & 95 & $25 \%$ & $26 \%$ \\
\hline DefazioSouthRiverEastBridge & 566 & 682 & 683 & $20 \%$ & $21 \%$ \\
\hline DeltaBPBridgeEastGoodPasture & 207 & 262 & 263 & $27 \%$ & $27 \%$ \\
\hline EastbankSouthOwossoBr & 488 & 388 & 389 & $20 \%$ & $20 \%$ \\
\hline EWEBPathEast5th & 76 & 158 & 158 & $108 \%$ & $107 \%$ \\
\hline EWEBPathEastPioneerPkwy & 47 & 94 & 95 & $100 \%$ & $101 \%$ \\
\hline FernRidgeEastDanebo & 178 & 273 & 273 & $53 \%$ & $53 \%$ \\
\hline FirEastRiverRd & 319 & 315 & 315 & $1 \%$ & $1 \%$ \\
\hline FriendlySouth18th & 217 & 357 & 357 & $65 \%$ & $65 \%$ \\
\hline GatewayBPBridgeEastl5 & 95 & 231 & 231 & $143 \%$ & $143 \%$ \\
\hline GatewayStreetEastl5 & 42 & 52 & 53 & $24 \%$ & $25 \%$ \\
\hline GoodpastureEastDeltaHwy & 149 & 200 & 200 & $34 \%$ & $34 \%$ \\
\hline GWestMohawk & 112 & 147 & 147 & $31 \%$ & $31 \%$ \\
\hline HarlowEastl5 & 55 & 84 & 84 & $53 \%$ & $53 \%$ \\
\hline HeronBridgeSouthFernRidge & 372 & 598 & 599 & $61 \%$ & $61 \%$ \\
\hline HighNorth13th & 299 & 704 & 704 & $135 \%$ & $135 \%$ \\
\hline HighSouth4th & 718 & 1040 & 1040 & $45 \%$ & $45 \%$ \\
\hline MonroeSouth8th & 478 & 504 & 504 & $5 \%$ & $5 \%$ \\
\hline
\end{tabular}




\begin{tabular}{|c|c|c|c|c|c|}
\hline NorthbankEastKnickerbocker & 596 & 640 & 641 & $7 \%$ & $7 \%$ \\
\hline NorthbankSouthGreenwayBr & 509 & 483 & 483 & $5 \%$ & $5 \%$ \\
\hline NorthbankWestDeltaHwy & 338 & 220 & 221 & $35 \%$ & $35 \%$ \\
\hline PearlSouth19th & 119 & 116 & 116 & $3 \%$ & $3 \%$ \\
\hline PioneerPkwySouthQ & 190 & 158 & 158 & $17 \%$ & $17 \%$ \\
\hline RichardsonBridge & 427 & 672 & 672 & $57 \%$ & $57 \%$ \\
\hline SouthbankSouthGreenwayBr & 1098 & 1166 & 1166 & $6 \%$ & $6 \%$ \\
\hline ThurstonEast58th & 44 & 158 & 158 & $259 \%$ & $258 \%$ \\
\hline UniversityNorth24th & 220 & 294 & 294 & $34 \%$ & $34 \%$ \\
\hline WillametteNorth15th & 330 & 273 & 273 & $17 \%$ & $17 \%$ \\
\hline
\end{tabular}


Table 3.31 - Comparison of Scenario 1 and Scenario 2 PM NBPDP Factors

\begin{tabular}{|c|c|c|c|c|c|}
\hline \multicolumn{6}{|c|}{ Comparison of Scenario 1 and Scenario 2 PM NBPDP Factors } \\
\hline Location & Observed & $\begin{array}{l}\text { Scenario } \\
1 \\
\text { Estimate }\end{array}$ & $\begin{array}{l}\text { Scenario } \\
2 \\
\text { Estimate }\end{array}$ & $\begin{array}{l}\text { Scenario } 1 \\
\text { Absolute \% } \\
\text { Difference }\end{array}$ & $\begin{array}{l}\text { Scenario } \\
2 \\
\text { Absolute } \\
\% \\
\text { Difference }\end{array}$ \\
\hline 15thWestJefferson & 798 & 1302 & 1302 & $63 \%$ & $63 \%$ \\
\hline AlderNorth27th & 702 & 1036 & 1036 & $48 \%$ & $48 \%$ \\
\hline AmazonPathNorth24th & 1023 & 1533 & 1533 & $50 \%$ & $50 \%$ \\
\hline DEastPioneerPkwyPath & 75 & 133 & 133 & $77 \%$ & $78 \%$ \\
\hline DefazioSouthRiverEastBridge & 566 & 875 & 875 & $55 \%$ & $55 \%$ \\
\hline DeltaBPBridgeEastGoodPasture & 207 & 483 & 483 & $133 \%$ & $133 \%$ \\
\hline EastbankSouthOwossoBr & 488 & 994 & 994 & $104 \%$ & $104 \%$ \\
\hline EWEBPathEast5th & 76 & 98 & 98 & $29 \%$ & $28 \%$ \\
\hline EWEBPathEastPioneerPkwy & 47 & 84 & 84 & $79 \%$ & $79 \%$ \\
\hline FernRidgeEastDanebo & 178 & 301 & 301 & $69 \%$ & $69 \%$ \\
\hline FirEastRiverRd & 319 & 420 & 420 & $32 \%$ & $32 \%$ \\
\hline FriendlySouth18th & 217 & 357 & 357 & $65 \%$ & $65 \%$ \\
\hline GatewayBPBridgeEastl5 & 95 & 189 & 189 & $99 \%$ & $99 \%$ \\
\hline GatewayStreetEastl5 & 42 & 14 & 14 & $67 \%$ & $68 \%$ \\
\hline GoodpastureEastDeltaHwy & 149 & 175 & 175 & $17 \%$ & $18 \%$ \\
\hline GWestMohawk & 112 & 112 & 112 & $0 \%$ & $0 \%$ \\
\hline HarlowEastl5 & 55 & 56 & 56 & $2 \%$ & $1 \%$ \\
\hline HeronBridgeSouthFernRidge & 372 & 595 & 595 & $60 \%$ & $60 \%$ \\
\hline HighNorth13th & 299 & 315 & 315 & $5 \%$ & $5 \%$ \\
\hline HighSouth4th & 718 & 840 & 840 & $17 \%$ & $17 \%$ \\
\hline MonroeSouth8th & 478 & 616 & 616 & $29 \%$ & $29 \%$ \\
\hline
\end{tabular}




\begin{tabular}{|l|r|r|r|r|r|} 
NorthbankEastKnickerbocker & 596 & 973 & 973 & $63 \%$ & $63 \%$ \\
\hline NorthbankSouthGreenwayBr & 509 & 1071 & 1071 & $110 \%$ & $110 \%$ \\
\hline NorthbankWestDeltaHwy & 338 & 553 & 553 & $64 \%$ & $64 \%$ \\
\hline PearlSouth19th & 119 & 147 & 147 & $24 \%$ & $24 \%$ \\
\hline PioneerPkwySouthQ & 190 & 259 & 259 & $36 \%$ & $37 \%$ \\
\hline RichardsonBridge & 427 & 707 & 707 & $66 \%$ & $65 \%$ \\
\hline SouthbankSouthGreenwayBr & 1098 & 1981 & 1981 & $80 \%$ & $80 \%$ \\
\hline ThurstonEast58th & 44 & 35 & 35 & $20 \%$ & $21 \%$ \\
\hline UniversityNorth24th & 220 & 322 & 322 & $46 \%$ & $47 \%$ \\
\hline WillametteNorth15th & 330 & 364 & 364 & $10 \%$ & $10 \%$ \\
\hline
\end{tabular}


A comparison of the average absolute percentage difference is presented in Table 3.30 to help understand the overall outcomes and the differences between the different estimation scenarios. The table demonstrates that Scenario 2 factors produced estimates with less error on average compared to Scenario 1 factors for locations tested in the initial Scenario 1 factor implementation. The results for the NBPDP did not change since those factors were not altered for either scenario nor id the locations where factors were applied. The study area PM factors again produced the least amount of error. The results from the 32 locations resulted in slightly higher error with $16 \%$ compared to the full set of 123 locations that Scenario 2 factors were applied to above which resulted in average absolute error of $15 \%$.

Table 3.32 - Comparison of Scenario 1 and Scenario 2 Average Absolute Percentage Difference

\begin{tabular}{|l|c|c|}
\hline \multicolumn{3}{|c|}{$\begin{array}{l}\text { Comparison of Scenario } 1 \text { and Scenario 2 } \\
\text { Average Absolute Percentage Difference }\end{array}$} \\
\hline Factors & Scenario 1 & Scenario 2 \\
\hline Study Area AM & $34 \%$ & $29 \%$ \\
\hline Study Area PM & $20 \%$ & $16 \%$ \\
\hline NBPDP AM & $52 \%$ & $52 \%$ \\
\hline NBPDP PM & $53 \%$ & $53 \%$ \\
\hline
\end{tabular}




\section{Discussion and Conclusion}

\section{1 - Introduction}

Applying time-of-day factors to expand two-hour counts to estimated full day counts can be an effective way to make bicycle count programs with limited resources produce more meaningful bicycle traffic counts provided those estimates are reliable. The examinations completed for this research tested different time-of-day factors created from study area data and compared them to NBPDP factors in order to demonstrate the possible error from each application. Estimates from study area factors continuously out performed estimates derived from NBPDP factors. Factors from the NHTS and OHAS travel surveys would likely produce poor results since they do not conform to either Scenario 1 or Scenario 2 factors and factor groupings are impossible to form from the survey data available. Evaluation of the study area data and the revelation of a university travel pattern is interesting but does not help with time-of-day factoring since the peak period factors do not compare well for locations with a possible university travel designation.

\section{2 - NHTS and OHAS Derived Factors}

The factors based on NHTS and OHAS travel surveys do not appear capable of producing reliable results in all cases when applied in an extrapolation exercise. Though no test of their application was performed, a review of the factor values compared to 
those in Scenario 1 and Scenario 2 reveal that the NHTS and OHAS values are different in many instances. For the NHTS factors, the AM factor is identical to the Scenario 1 path factor group but since the rest of the factor group factors vary estimates would result in some error. The OHAS factors also result in some overlap with some of the study area scenario factors. The OHAS AM factors are all much higher compared with AM study area factors but the OHAS-Eugene PM factor is identical to the study area scenario 2 Blvd and No Facility factors. Some testing could be done applying these factors but without bicycle facility specific groupings estimates are likely to contain high levels of error. Based on the review of NHTS and OHAS factors for this research, it is not recommended they be used as time-of-day factors.

\section{3 - Review of the University Bicycle Travel Pattern}

Using study area data a university travel patterns is suggested with proposed characteristics to identify this pattern. Identification of this travel pattern would be useful to planners looking to understand users of a particular facility but would not be helpful in constructing factors or factor groupings since no trend revealed itself in the AM or PM peak factors for locations characterized as exhibiting university travel patterns. Understanding user types by identifying bicycle travel patterns is important when considering how bicyclists may be interacting with motorized travel which could inform how future improvements might occur. For instance, knowing that bicycle travel around a university is more or less steady throughout the day instead of being diurnal 
like many transportation facilities with commute patterns could help make the case for completely separated bicycle infrastructure. The identification of this travel pattern is important but there is a need to develop additional attributes like weekday and weekend daily volumes to characterize this traffic pattern.

\section{4 - Study Area Time-of-Day Factors Application}

The results from study area time-of-day factors consistently outperformed NBPDP factors, especially the estimates derived from PM factor application. This outcome is important to those looking to apply the NBPDP to locally collected two-hour manual counts. It was not known how well the NBPDP factors performed in a given area as no test was previously done with observed 24 -hour bicycle counts. An important takeaway is that NBPDP factors have the potential for significant error in a given application.

Factors from Scenario 2 produced results with less error compared to Scenario 1 but required more data to create. As described above, an iterative process was created that tested various factors by factor group to determine the best factors to apply. Creating factors from this method would require interested parties to collect a larger number of 24-hour counts that would then be fed into an iterative testing process in order to determine the factors that produce the least amount of error. This research used 123 24-hour observations of data from $40+$ locations which is a great deal of data to collect. This process is conceivable if private contractors made such services (collecting 24-hour bicycle counts) available but might be too difficult for other agencies without a lot of 
resources for their non-morotized traffic data collection programs. The same approach could be taken with factors derived in Scenario 1, though with less data feeding the factor creation process more error is likely.

Basing factor groups on bicycle facility type helped to minimize error and is a convenient way to classify factors. Interestingly, the AM study area factors for both Scenario 1 and Scenario 2 exhibit less variation between groups compared to those in the PM factors. The AM factors also produce more error than the PM factors however. The Scenario 2 study area factor application saw the addition of separate off-street path factor groups, one for paths with more commute users and another for recreational users. This additional factor grouping produced better results for path facilities. For this research the designation was done based on observations of 24-hour distributions which requires some kind of automated counter up front though its possible to make such a characterization based on minimal manual observations.

The results and discussion have described the error associated with each set of factors and their application in terms relative to one another, but how much error is reasonable? Lindsey et al. (2007) reported results for error in estimating monthly and annual figures at between $-6.2 \%$ to $-31.6 \%$ and $-20.2 \%$ to $-36.4 \%$ respectively. Nordback et al. (2012) suggest acceptable error from using short term counts to estimate annual average daily traffic may be around $15 \%-30 \%$. Both of these studies used short-term counts to estimate an annual traffic counts. The best estimates this research was able 
to generate resulted in error of between $0-71 \%$ with an average of $16 \%$. Some of the less accurate estimates come from locations where low daily traffic was observed and could very well be removed from the results for the reason described above regarding hourly variability (though a few fairly reliable estimates came form locations with less than 100 daily bicyclists). Removing these outliers, the average absolute percentage difference is $14 \%$ for the Scenario 2 PM study area factors. Is this level of error acceptable?

The level of acceptable error might differ depending on the application of the results. If the results are used to describe a daily estimate of traffic perhaps they are acceptable. What if they are used in additional extrapolation procedure to estimate annual traffic? A rough calculation using some test day-of-week and monthly factors can give an idea as to how much error might accumulate for an annual estimate using a daily estimate with some initial error. Annual estimates will be compared based on the method for estimating their daily traffic.

Even though the NBPDP factors have proven themselves as unreliable for the study region, for the purposes of estimating annual bicycle traffic, the NBPDP day-of-week and monthly factors will be applied to a small number of time-of-day estimates to determine how daily estimate error might compound for annual traffic estimates. The NBPDP recommends using 0.13 and 0.12 day-of week factors for Tuesday and Thursday 
respectively. They also recommend using 0.06 as a monthly factor for counts collected in October and November. Therefore the annual estimation equation will be as follows:

\section{Figure 4.1 - Annual Bicycle Traffic Estimation Equation AnnualTraffici $=($ DailyEstimate $/$ WeeklyFactor $/$ MonthlyFactor $))$}

where AnnualTraffic is the total yearly bicycle traffic estimate, DailyEstimate is the estimate from the time-of-day factor application, WeeklyFactor is the day-of-week factor from NBPDP described above and the MonthlyFactor is the monthly factor from NBPDP described above. Applying this equation to just one location where the daily estimate varied between study area factors and NBPDP factors, the difference of yearly bicycle traffic will be presented. This quick examination will use a daily estimate from the Frohnmayer South River location which when using the study area Scenario 2 PM factors resulted in almost no error but when applying the NBPDP factors results in $47 \%$ error. The results from this annual extrapolation are presented below. 
Table 4.1 - Annual Estimate pf Bicycle Traffic Results

\begin{tabular}{|l|r|r|l|l|l|}
\hline & Location & $\begin{array}{l}\text { Absolute } \\
\text { Percent } \\
\text { Difference(Time- } \\
\text { of-Day Factor } \\
\text { Estimate) }\end{array}$ & $\begin{array}{l}\text { Annual } \\
\text { Estimate }\end{array}$ & $\begin{array}{l}\text { Factor } \\
\text { Type }\end{array}$ \\
\hline FrohnmayerSouthRiver & 1686 & 1691 & & $\begin{array}{l}\text { Study Area } \\
\text { PM } \\
\text { Scenario 2 }\end{array}$ \\
\hline FrohnmayerSouthRiver & 2478 & 1691 & $0 \%$ & 23,417 & $\begin{array}{l}\text { NBPDP } \\
\text { PM }\end{array}$ \\
\hline
\end{tabular}

The annual result error does not necessary compound the daily estimate result using the above day-of-week and monthly factors as the NBPDP derived daily estimate results in a $47 \%$ higher annual estimate which is identical to the original time-of-day factor derived result. However, the total bicyclist difference is 11,000 which would have some major implications in any analysis that used these figures as their basis for bicycle travel. Any kind of public health or green house gas analysis using these numbers would vastly over estimate the benefits from bicycle travel using the NBPDP derived estimate. The implications would be similar for a safety analysis where bicyclist's crash data were used in conjunction to this annualized estimate. More work needs to be done to compare how estimates derived from factoring affect the uses of those estimates. Minimizing error should be the goal in all factor development and application of factors whether they are time-of-day, day-of-week, or monthly.

It is possible that additional factor groups are needed to further refine the estimation results. One issue is how to best describe the factor group without count data to support the classification. Bike facility makes for an easy factor group because it 
only requires that the facility type is known but this may not get at the user differences adequately, as was seen in the off-street path factor group between Scenario 1 and 2. More testing could be done using attributes of urban form in and around the count location to establish more factor groups though that does take more data which might not be available to all users.

\section{5 - Research Limitations}

It is important to restate that the study area factors were constructed using only Tuesday and Thursday data. Tuesday and Thursday average daily volumes and hourly distributions are similar and remove some of the variation that would likely cause more error if different days of the week were used. Some counts from the summer had multiple days of the week but because of the structure of the count program from which this data was collected, Tuesday and Thursdays are the only days collected for nearly all the locations. The factors presented above then are AM and PM peak period factors for Tuesday and Thursday and would likely be different for other days of the week, especially weekends when bicycle travel is primarily recreational. This would imply that hourly patterns would probably change for all factor groups.

Another limitation to this research is the application of NBPDP for all types of bicycle facilities. The NBPDP factors are only designed to be applied to either a pedestrian district or an off-street path facility, though no explicit guidance against doing so is 
found on in the NBPDP documentation. Additionally, of the reports that applied NBDPD factors, many counts came from non-path facility types.

The product of using short-term two-hour counts to estimate 24-hour volumes is not the most useful result. As the NBPDP factoring methodology does provide factors to extrapolate short-term counts to weekly, and ultimately monthly, and yearly volumes, any factors developed from local data should also look to maintain the capability to estimate longer term, ideally, annual, traffic counts. Some analysis tools like the World Health Organization's Health Economic Assessment Tools (HEAT) instrument prefer longer term counts that represent average traffic levels (WHO 2011). Additionally, annual volumes tell a more compelling story to the public and to policy makers that are looking at how best to spend scarce public dollars. Though daily estimates from shortterm counts are not the most compelling, they may still be an important metric for traffic if more agencies continue to only collect two-hour counts.

This research did attempt to refine the time-of-day estimation process by splitting the typical practice of summing both directions of travel and estimating each direction separately. This led to some improvement in locations that were one-way but overall did not significantly improve estimation results.

\section{6- Conclusion}

Having the ability to use short-term two-hour bicycle counts to reliably estimate full day 24-hour counts allows transportation analysts and planners the ability to use fewer 
resources to get more information they may need for both short-term and long-term planning goals. Having reliable time-of-day factors to estimate daily traffic would reduce error in any analysis where daily traffic estimates are employed. This research tested time-of-day factors developed from study area bicycle counts and compares the results from applying factors from the NBPDP, and found that study area factors consistently out perform NBPDP factors. Factors developed in Scenario 2 from study area data produced results with the least amount of error though it would be up to those wishing to apply factors in a similar way to determine how much error is acceptable. NBPDP factors do not appear to be deployable in the study area without acceptance of levels of error that might mis-characterize the location's level of bicycle traffic. Other areas looking to augment their own two-hour counts by applying the NBPDP factors should do so with caution and this research recommends some kind of validation of the factors similar to what was done for this research. Creating factor groupings on bicycle facility and users is a convenient way to refine factors that help reduce error from 24-hour estimates.

Reliability of estimates was reduced when counts with less than 100 daily riders were observed. This finding would suggest that hourly distributions for these kinds of locations are highly variable and that when locations with low daily traffic are are counted using manual data, multiple 2-hour counts should be collected and averaged in order to reduce some of the variation. This recommendation is supported in the NBPDP 
documentation where they suggest averaging multiple 2-hour manual counts and report the average.

This work establishes the university travel activity pattern using study area data which may help other areas when they think about how to classify behavior on bicycle facilities. Though not useful for time-of-day factoring, the university travel pattern might be useful for monthly or annual factoring processes. More work should be done in this area. This research also examined available data sets including national and statewide travel survey data in order to assess the ability of those data sources to determine usable time-of-day factors, finding these data sources do not produce usable factors.

For practitionaers and others thinking about how to start a count program, the NBPDP offers good guidance on how to begin data collection but the use of their factors should be limited. If manual counts do not provide enough information and automatic technology can be acquired, a great deal more about bicycle traffic can be learned. Using 24-hour count data collected from automatic devices, time-of-day factors can be derived based on factor groupings that are easily constructed which can then be used to augment any on going or future manual count program. Even without applying factors, so much more information can be acquired with a limited number of automatic movable counting devices. The CLMPO was able to collect all of the data used in this research with just four movable tube counters. 
As the funding sources increase for non-motorized traffic, it should become easier to justify spending money on tracking bicycle traffic changes. Two-hour counts provide some useful information about traffic levels but they do not supplant the resolution of full day or week counts. The ultimate goal should be permanent counters that detail each hour of each day over the course of an entire year. As bicycle traffic counting become more common and the investments in bicycle infrastructure can be demonstrated, more investment can be substantiated, which wouldcreate a feedback loop that will help supply the many benefits of bicycling to a growing number of people. Bicycle count programs have begun all over the country, and some, like those in Boulder, Colorado, Minneapolis, Minnesota, and San Diego California, have also installed a large number of permanent continuous automatic counters. As these programs mature and more like them come online, factoring methodologies should catch up to their motorized traffic counter parts, though likely not soon enough. Whether counts are used as supplemental inputs into safety planning or health impact assessments or simply as a performance measure to determine how well a particular areas' investment in bicycle infrastructure is performing, bike count data are vital in telling the story that U.S. Census data like journey to work is unable to tell. Some agencies and practitioners will be unable to afford counting equipment and will rely on manual counts collected by volunteers long into the future which will make reliable extrapolation factors increasingly important. As efforts such as the NPBPD develop, state departments of transportation and metropolitan planning organizations will 
hopefully gain a greater understanding of these estimation processes and will seek to provide locally relevant bicycle traffic count factors as many of them currently do for motorized traffic.

The benefits from increased bicycling, whether it is for utilitarian purposes like commuting to work or recreational trips, are becoming increasingly apparent with growing body of evidence. The public health, economic development and environmental benefits as well as energy savings from increased walking and biking are demonstratable. Funding bicycle and active transportation improvements in general will likely grow as they have over the last 20 years. According to the bicycle and walking advocacy group Advocacy Advance, federal funding for bicycle and pedestrian related projects has increase from less than $\$ 50$ million per year to over $\$ 600$ million a year not accounting for America Reinvestment and Recovery Act funding which would add over a billion dollars to this amount. (Advocacy Advance 2011) If the level of funding for active modes remains stable or continues to grow, planning agencies will need to better measure and understand travel demand for active transportation. Counting users is a necessary next step but updated travel demand models and other analystical tools is also necessary so that policy makers, planners, and the public have the right information to make good decisions about the transportation system. 


\section{Citations}

Barnes, G. and Krizek, K. Estimating Bicycle Demand. In Transportation Research Record: Journal of the Transportation Research Board of the National Academies 1939, Washington D.C., 2005, pp 45-51

Buffalo Valley Rail Trail Research Team at Bucknell University. Lewisburg Area Recreational Authority and Union County, Pennsylvania. Buffalo Valley Rail Trail 2012 User Survey and Economic Impact Analysis. August 2012

Dill, J., and T. Carr. Bicycle Commuting and Facilities in Major U.S. Cities: If You Build Them, Commuters Will Use Them. In Transportation Research Record: Journal of the Transportation Research Board, No. 1828, Transportation Research Board of the National Academies, Washington, D.C., 2003, pp. 116-123.

Central Lane Metropolitan Planning Organization, 2012 Summer and Fall Bicycle Counts Report, 2013.

http://www.thempo.org/documents/CLMPO RBCP Summer Fall 2012 Report.pdf (retrieved January 2013)

Chicago Department of Transportation. 2009 Bike Counts Project. 2009. http://www.cityofchicago.org/content/dam/city/depts/cdot/bicycling/CDOT bicycle co unt study 2009.pdf (retrieved December 2012)

City of Eugene: Bicycle and Pedestrian Master Plan. March 2012.

http://www.centrallanertsp.org/sites/default/files/Eugene\%20PBMP\%20Final\%20small. pdf (retrieved December 2012)

City of Portland Bureau of Transportation. 2011 Bicycle Counts Report. February 2012. www.portlandoregon.gov/transportation/article/386265 (retrieved December 2012)

City of Springfield: Springfield Bicycle Plan. June 1998.

http://www.ci.springfield.or.us/pubworks/EngineeringTransportation/documents/Sprin gfieldBicyclePlan.pdf (retrieved December 2012)

City of Toronto: Cycling Infrastructure and Programs Transportation Services. 2012 Bicycle Count Report. December 2010.

www.toronto.ca/cycling/reports/pdf/bicycle count report 2010.pdf (retrieved December 2012) 
Griswold, J. B., Medury, A., and Schneider, R.J. Pilot Model for Estimating Bicycle Intersection Volumes. In Transportation Research Record: Journal of the Transportation Research Board of the National Academies, Washington D.C., 2011, pp 1-7

Gulf Coast Research Center for Evacuation and Transportation Resiliency. Active Transportation Measurement and Benchmarking Development: New Orleans Pedestrian and Bicycle Count Report, 2010-2011. http://www.evaccenter.Isu.edu/pub/1105Part\%202.pdf (retrieved October 2012)

Dora, C. and M. Phillips (ed). Transport, Environment and Health. WHO Regional Publications, European Series, no 89, World health Organization, 2000.

Dill, J. Bicycling for Transportation and Health: The Role of Infrastructure. Journal of Public Health Policy 2009 30:95-110.

Eco-Counter Pneumatic Tube Documentation. Retrieved 2013. http://www.ecocompteur.com/Tubes.html?wpid=15040

Fairfax Advocates for Better Bicycling (FABB). "FABB Conducts First Volunteer Fairfax Bike Count". FABB Blog. August $5^{\text {th }}$ 2011. http://fabbbikes.blogspot.com/2011/08/fabb-conducts-first-volunteer-fairfax.html. Accessed January 2013

Fleisher, C.S., and D. Mahafy. A Balanced Scorecard Approach to Public Relations Management Assessment. Public Relations Review, Vol. 23, n. 2, 1997, pp.117-142.

Kuzmyak, J.R., Estimating Bicycling and Walking for Planning and Project Development. National Cooperative Highway Research Project 8-78. Completion June 2013

Jones M.G., Ryan S., Donlon J., Ledbetter L., Ragland D.R., Arnold L. Measuring Bicycle and Pedestrian Activity in San Diego County and its Relationship to Land Use, Transportation, Safety, and Facility Type. UC Berkeley Safe Transportation Research \& Education Center. Caltrans Task Order 6117 (2010)

Jones, M., S. Ryan, J. Donlon, L. Ledbetter, D. R. Ragland, and L. Arnold. Seamless Travel: Measuring Bicycle and Pedestrian Activity in San Diego County and its Relationship to Land Use, Transportation,Safety, and Facility Type. Caltrans Task Order 6117. California Department of Transportation, Berkeley, 2010. http://www.path.berkeley.edu/PATH/Publications/PDF/PRR/2010/PRR-2010-12.pdf. Accessed January 2013. 
Lindsey, G., Wilson, J., Rubchinskaya, E., Yang, J., and Han, Y. Estimating urban trail traffic: Methods for existing and proposed trails. Landscape and Urban Planning 2007, 81 (4), p. 299.

McRhodes, Shane, Safe Routes to School Coordinator for 4J School District (EugeneSpringfield Area) Personal Communication - 11/01/12

Mill Valley to Corte Madera Bicycle and Pedestrian Corridor Study, Appendix H: Use Counts and Projections. Prepared by Alta Planning. March 2012 (Accessed October 2012)

http://www.walkbikemarin.org/documents/mv cm study/FINAL\%20Study/Appendix\%2 $\underline{\text { OH.pdf }}$

Miranda-Moreno, L., \& Nosal, T. , T., Schneider, R.J., and Proulx, F. (2013). Classification of bicycle traffic patterns in five North American Cities Paper presented at the Transportation Research Board Annual Meeting.

Miranda-Moreno, L., \& Nosal (2011). Weather or not to cycle; whether or not cyclist ridership has grown: a look at weather's impact on cycling facilities and temporal trends in an urban environment. Paper presented at the Transportation Research Board Annual Meeting.

National Bicycle and Pedestrian Documentation Project http://bikepeddocumentation.org/ (retrieved October 2012)

Nordback, K., Marshall, W.E., Janson, B.N., and Stolz, E. Errors in Estimating Annual Average Daily Bicyclists from Short-term Counts. Practice Ready Paper In Transportation Research Record: Journal of the Transportation Research Board of the National Academies, Washington D.C., 2012

Office of the Auditor General Western Australia (OAG). Public Sector Performance Indicators 1993-94. Report n. 7, OAG, Australia, 1994.

Office of the Auditor General Western Australia (OAG). Preparing Performance Indicators:

A Practical Guide. 2. ed., OAG, Public Sector Management Office, Australia, 1997.

Oregon Department of Transportation. 2009 Oregon Household Activity Survey. http://www.oregon.gov/ODOT/TD/TP/pages/travelsurvey.aspx 
Regional Planning Commission for Jefferson, Orleans, Plaquemines, St. Bernard, and St. Tammary Parishes and the Louisiana Department of Transportation and Development. New Orleans Pedestrian and Bicycle Count Report 2012, July 2012.

http://transportation.uno.edu/phirecontent/assets/files/PBRI 2012 BikePed Count Report.pdf (accessed October 2012)

Roberts, I. et al., Pedalling Health-Health Benefits of a Modal Transport Shift, Bicycle Institute of South Australia (Sydney; www.science.adelaide.edu.au/slate/demos/cyhealth.pdf), 1996.

Sælensminde, K. Cost-Benefit analyses of walking and cycling track networks taking into account insecurity, health effects and external costs of motorized traffic. Transportation Research Part A: A Policy and Practice. Vol. 38, Issue 8, October 2004, Pages 593-606

San Francisco Municipal Transportation Agency. 2011 Bicycle Count Report. December 2010. http://www.sfmta.com/cms/rbikes/documents/2011BicycleCountReportsml.pdf

TR News - Active Transportation: Implementing the Benefits. Number 280 May-June 2012. Transportation Research Board of the National Academies. Accessed January 2013. http://onlinepubs.trb.org/onlinepubs/trnews/trnews280.pdf

University of Oregon 2013 Commuter Survey - Retrieved April 2013

U.S. Census Bureau; American Community Survey 5-year estimates, Table B08301; generated by Josh Roll; using American FactFinder; <<http://factfinder2.census.gov $>$; December 2012

U.S. Department of Transportation Federal Highway Administration. Pedestrian and Bicycle Data Collection in United States Communities Quantifying Use, Surveying Users, and Documenting Facility Extent. January 2005.

U.S. Department of Transportation Federal Highway Administration. Pedestrian and Bicycle Count Collection. January 2011.

U.S. Department of Transportation, Federal Highway Administration, 2009 National Household Travel Survey. URL: http://nhts.ornl.gov.

U.S. Department of Transportation Federal Highway Administration. Traffic Monitoring Guide. 2001. Retreived November 2012. 
Wang, Y., and N. L. Nihan. Estimating the Risk of Collisions Between Bicycles and Motor Vehicles at Signalized Intersections. Accident Analysis and Prevention, Vol. 36, 2004, pp. 313-321.

Wen LM, Rissel C. Inverse associations between cycling to work, public transport, and overweight and obesity: findings from a population based study in Australia. Prev Med. 2008; 46:29-32

World Health Organization. Health Economic Assessment Tools (HEAT) for Walking and for Cycling Methodology and User Guide, Economic Assessment of Transport Infrastructure And Polices. 2011 


\section{Appendix A - Seasonal Comparison of Hourly Distributions}
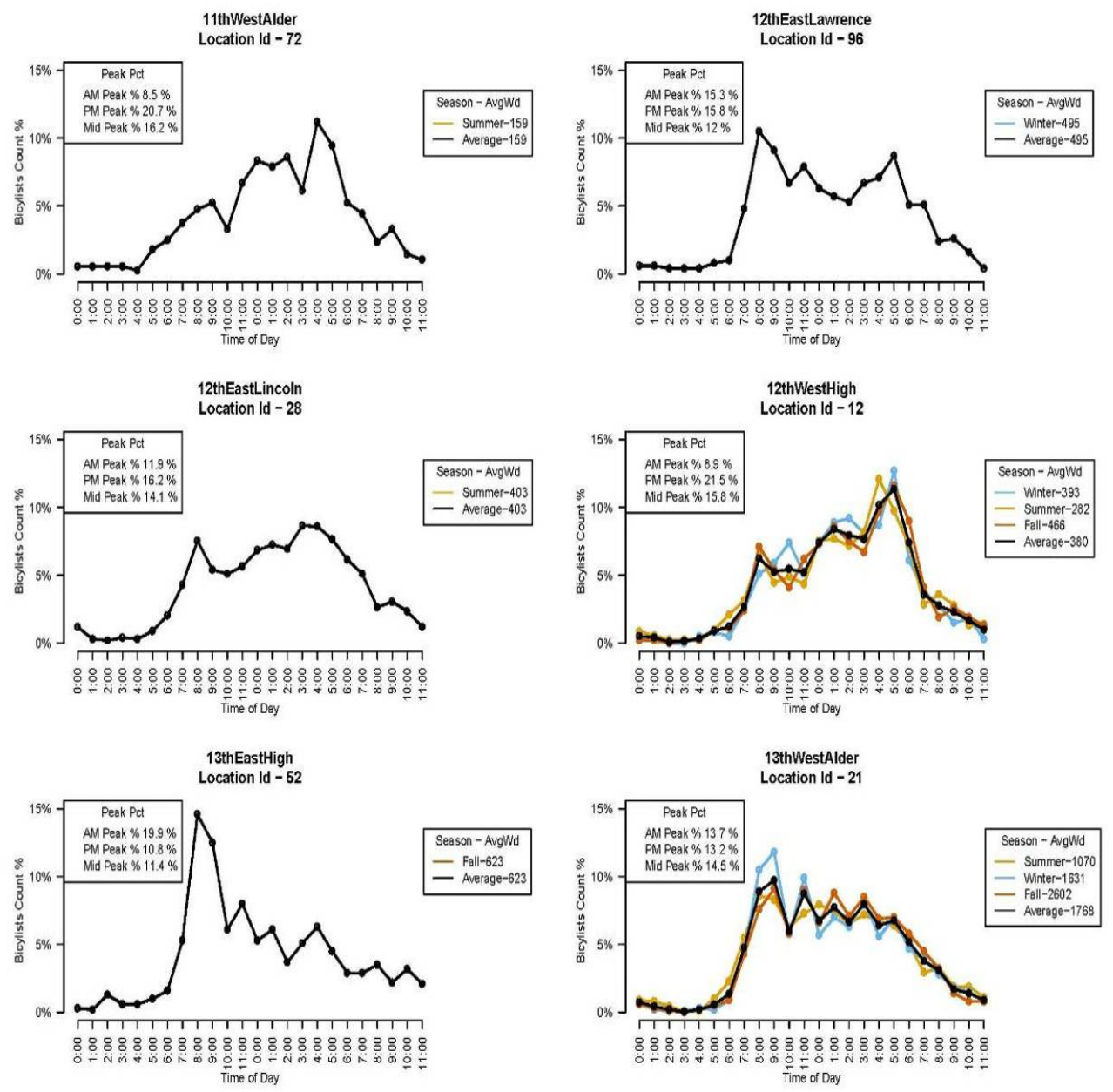

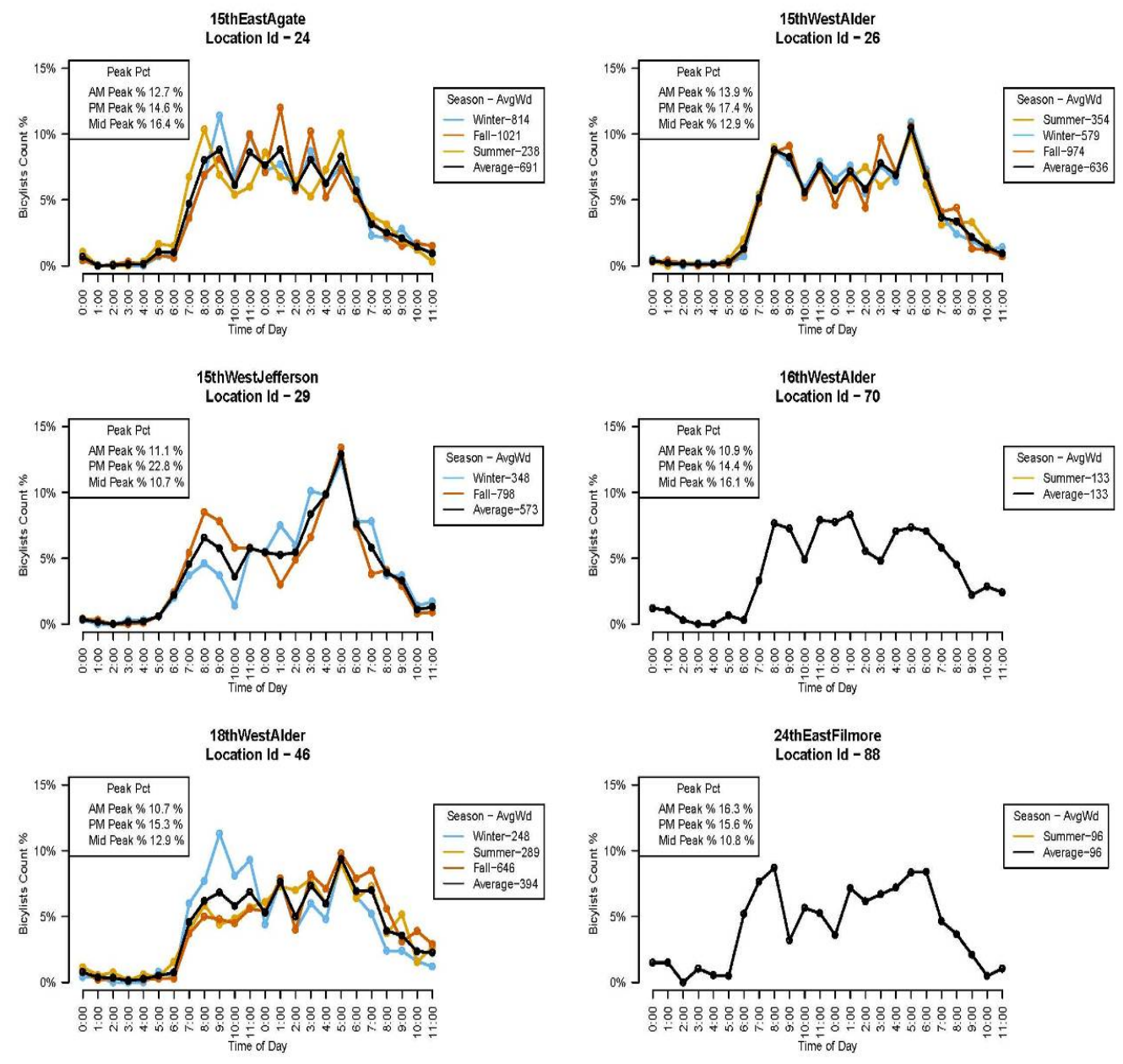

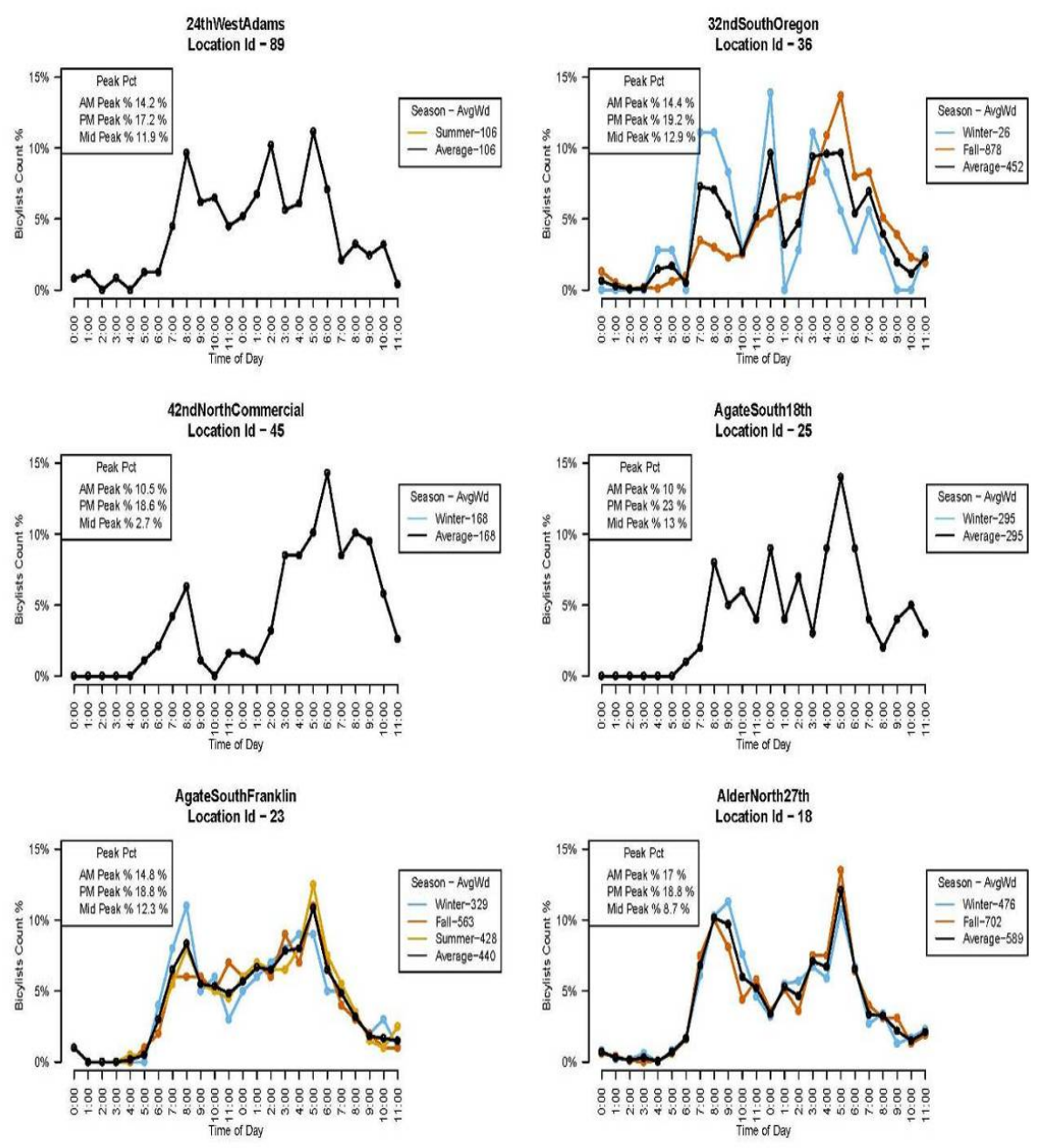

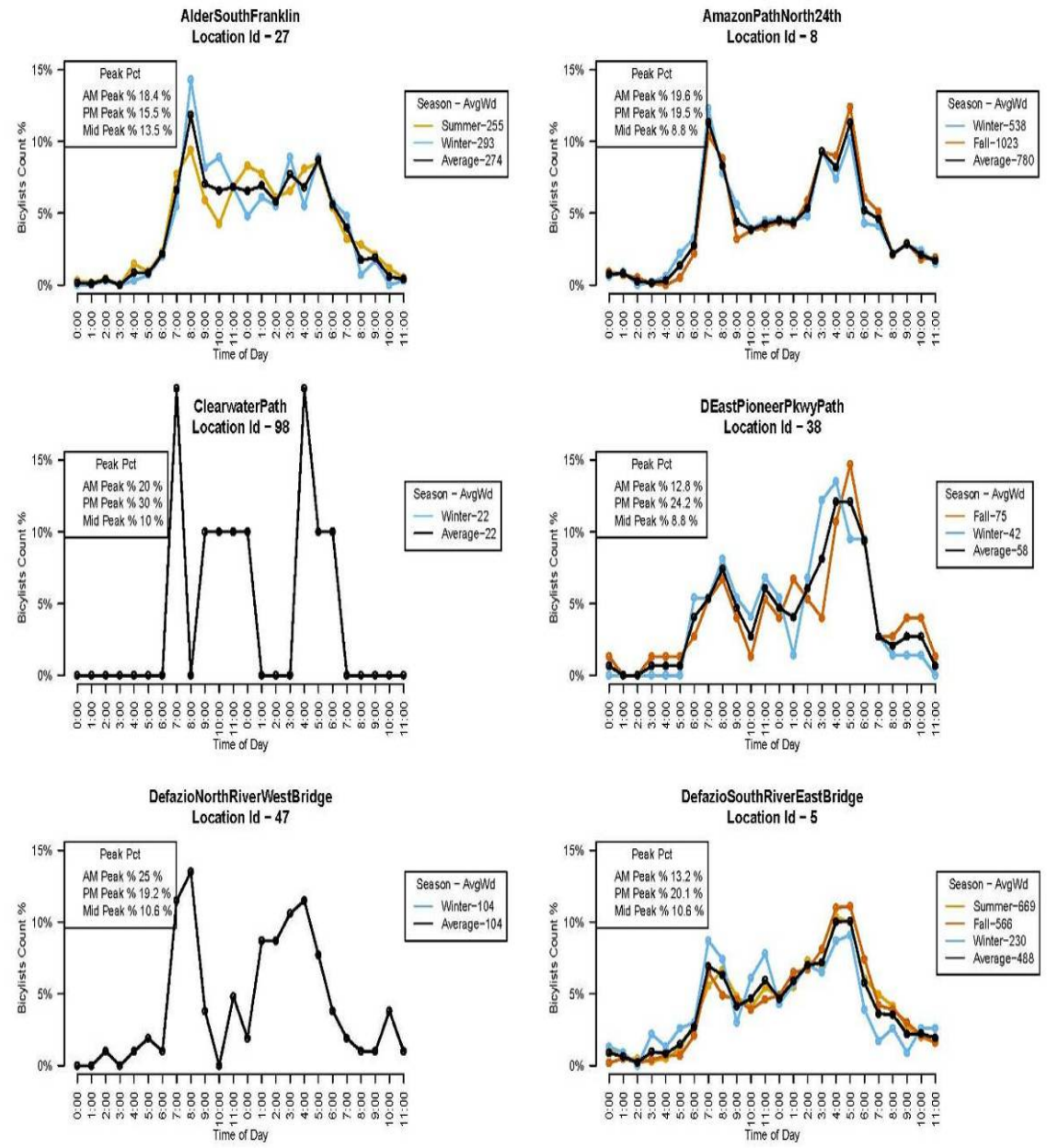

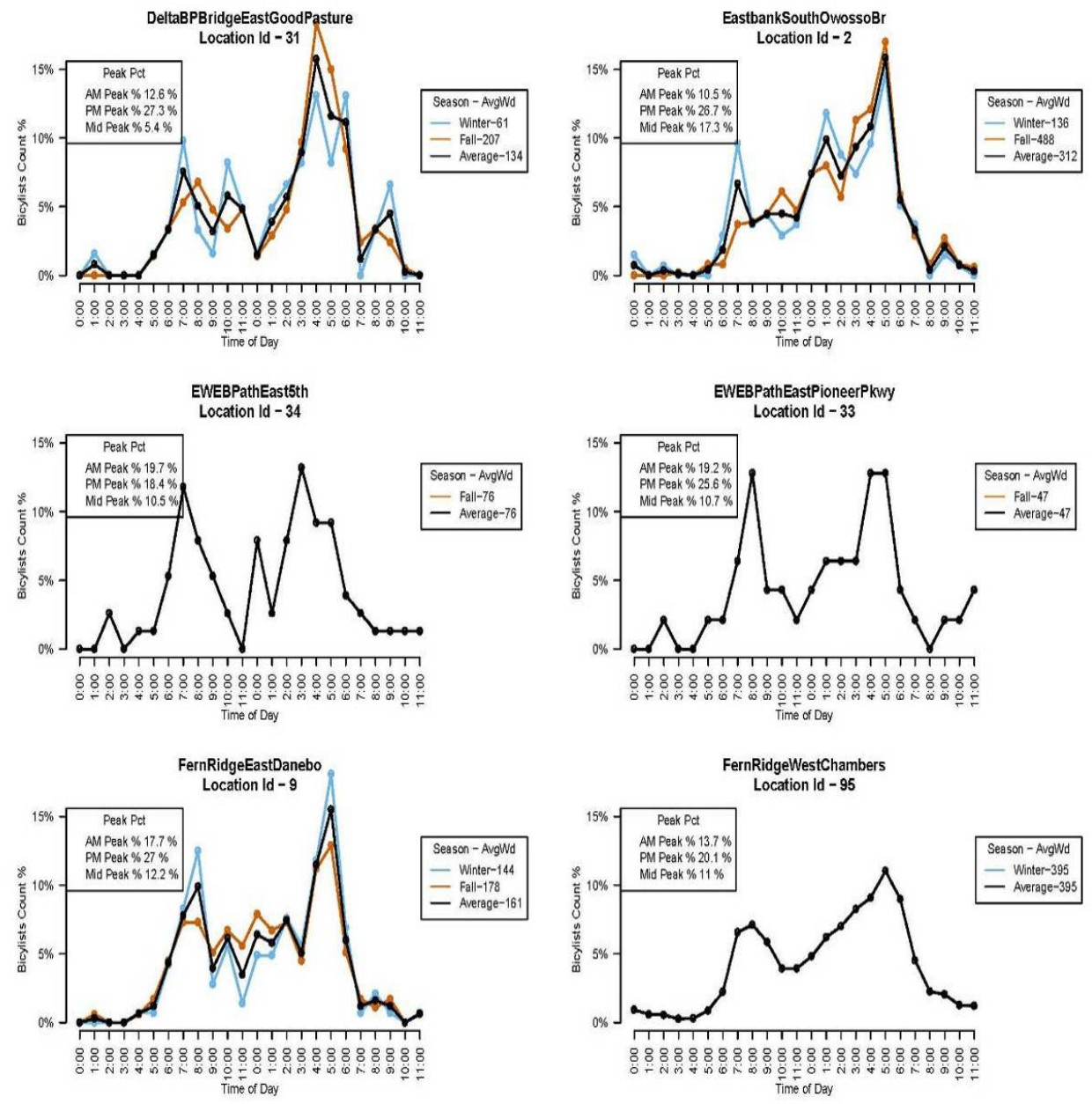

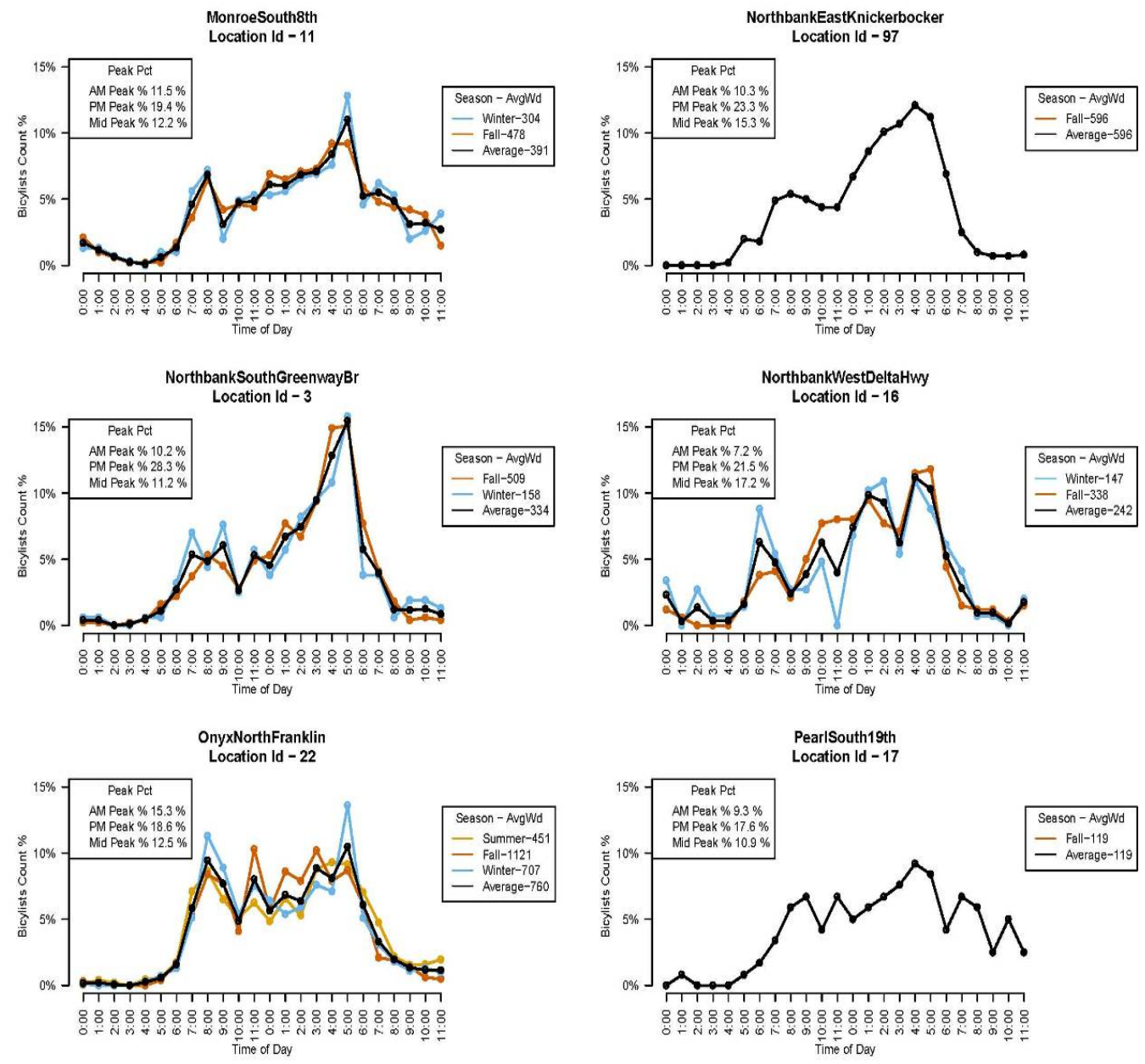

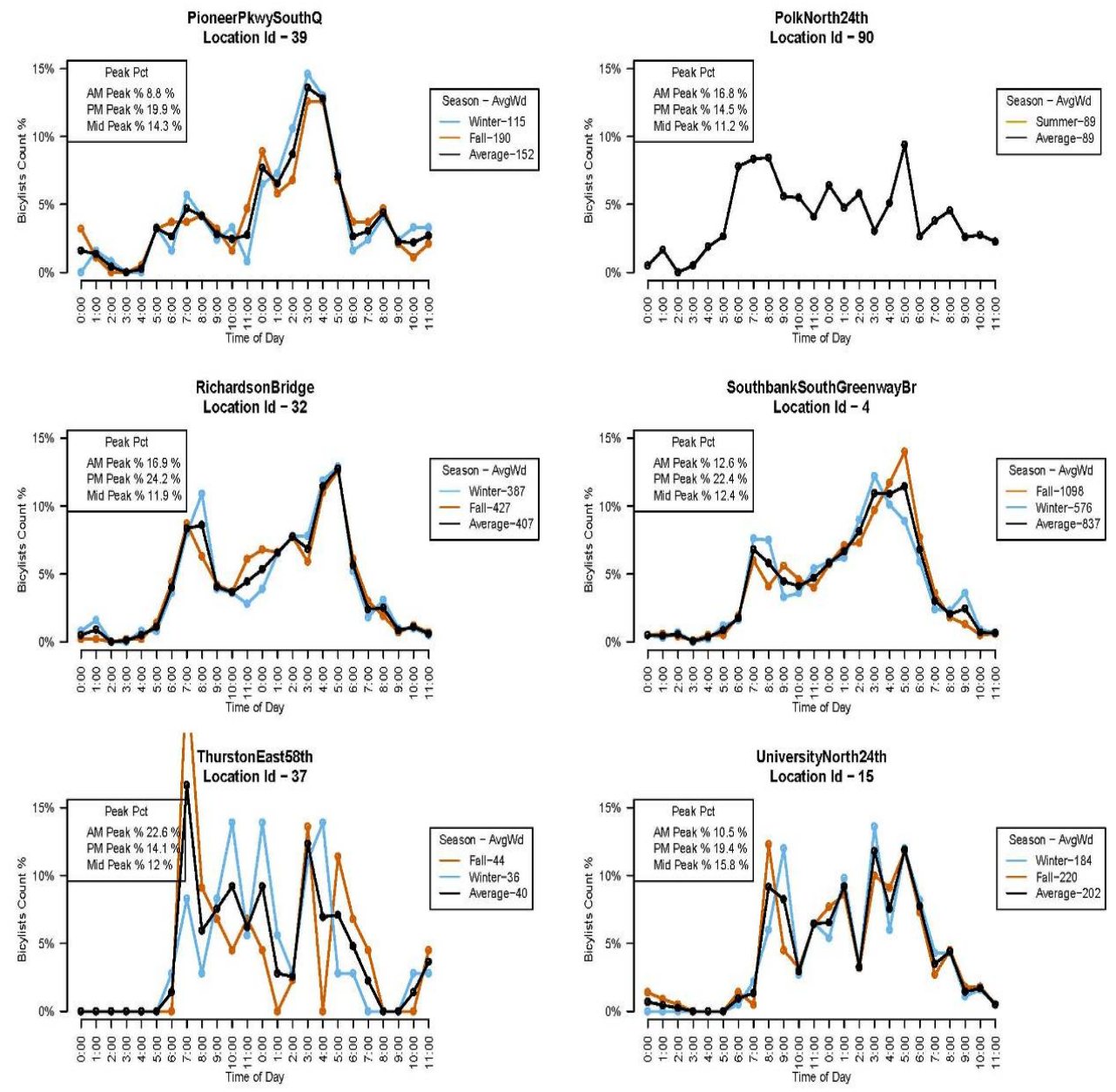

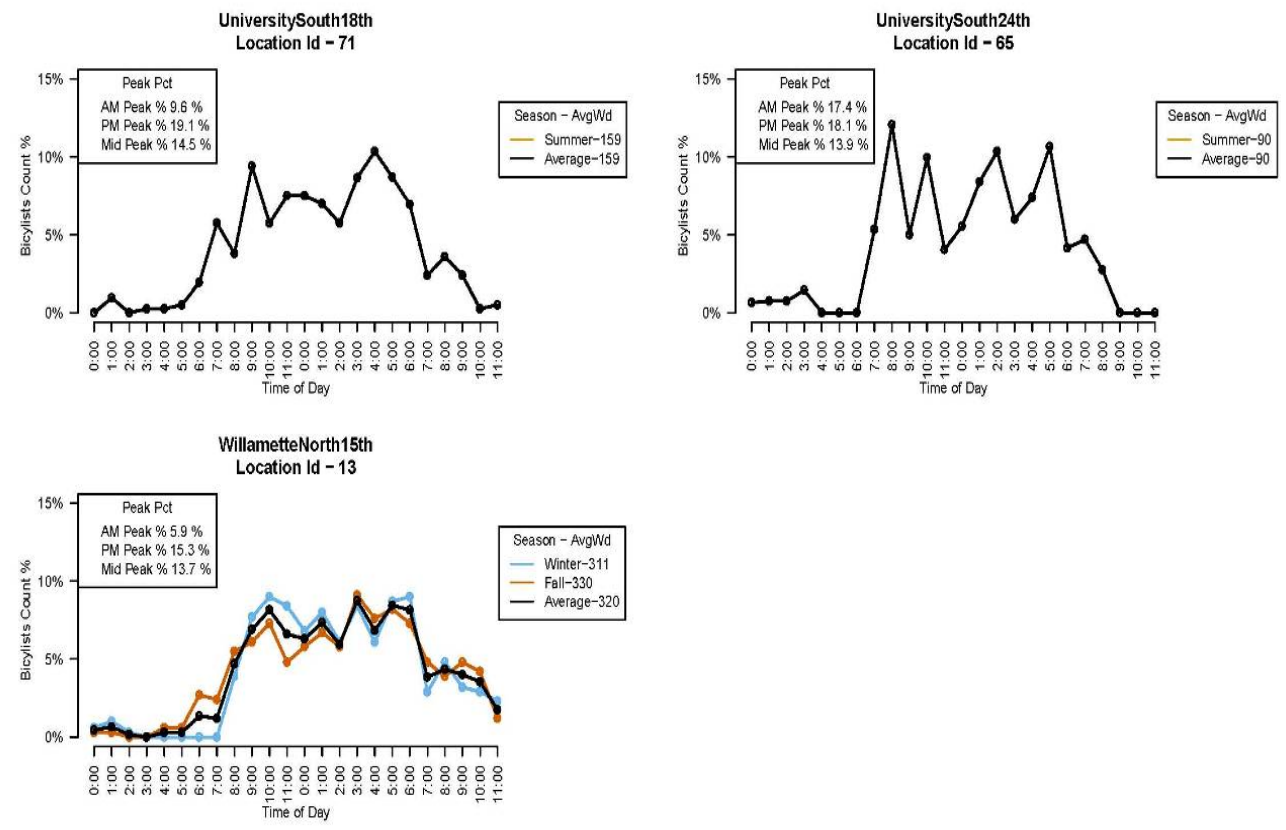

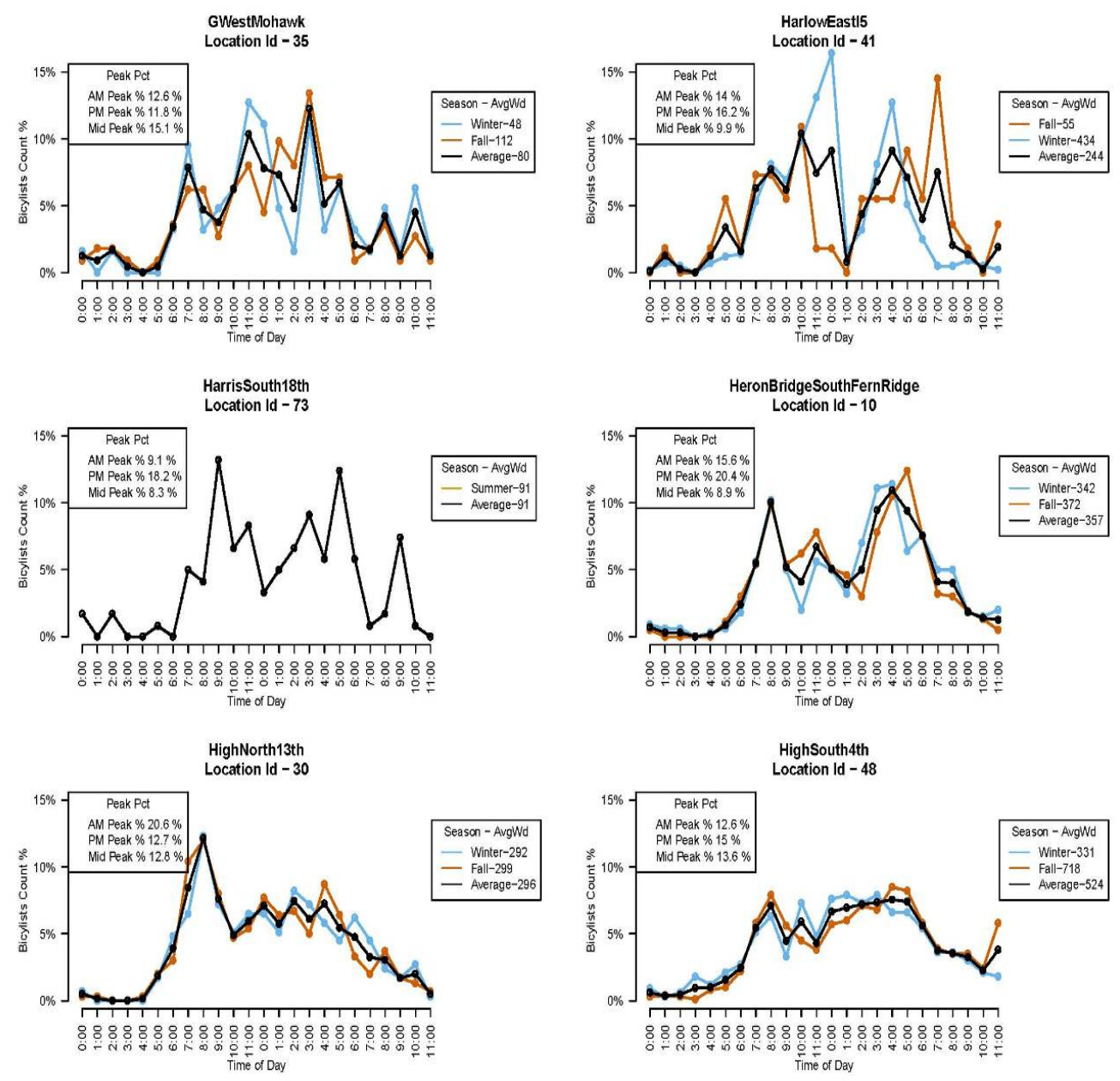


\section{Appendix B - Proportion of Weekly Travel by Day of Week}

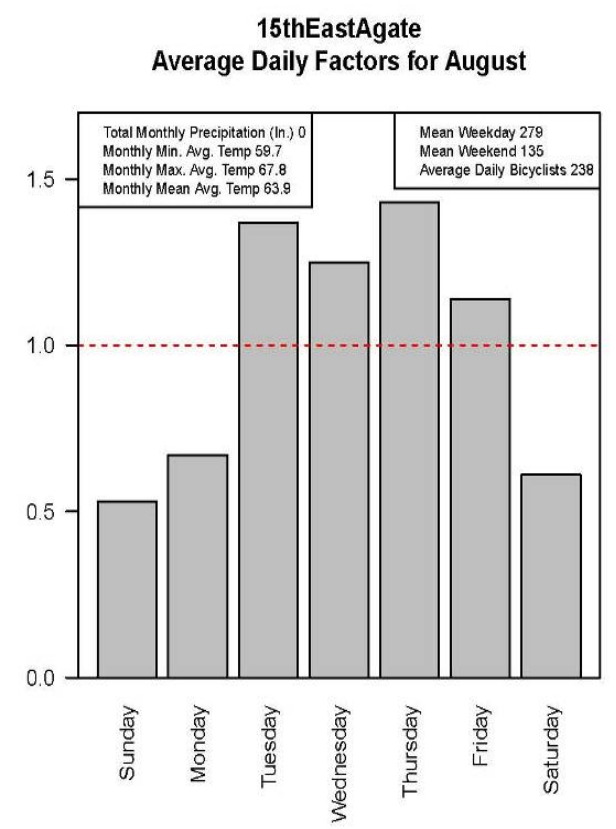

16thWestAlder Average Daily Factors for August

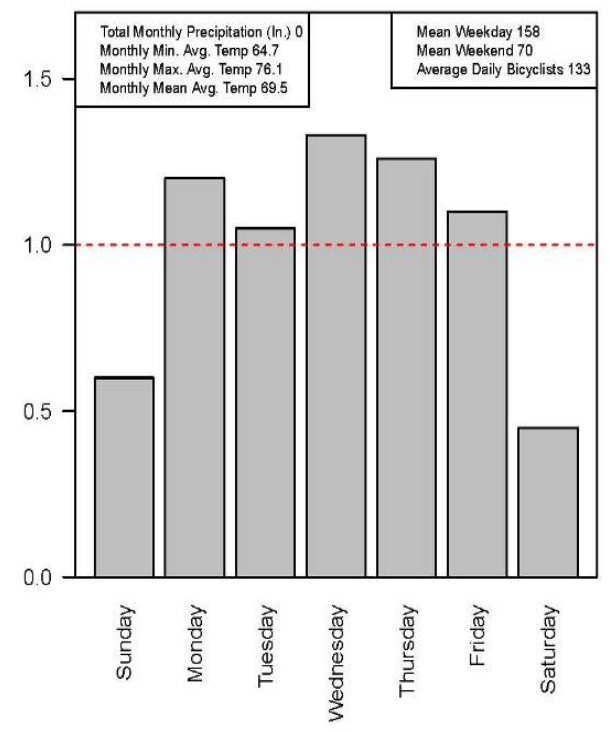

15thWestAlder

Average Daily Factors for July

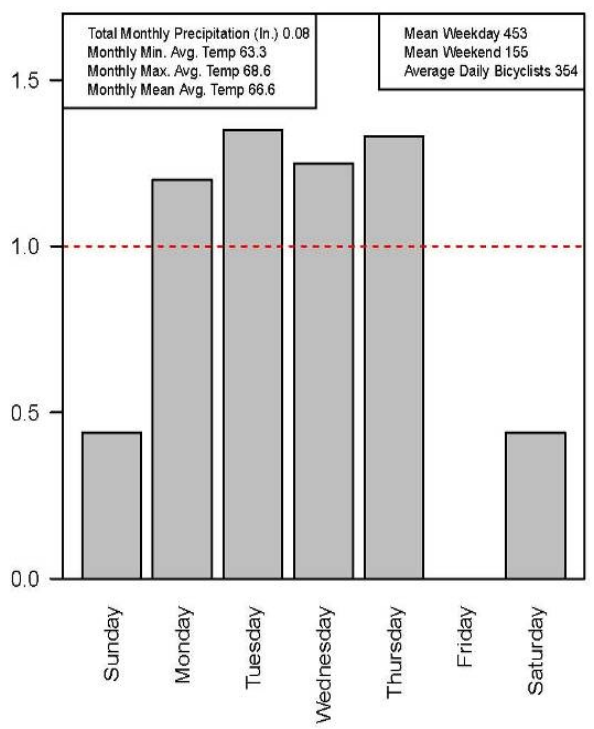

8thWestAlder Average Daily Factors for August

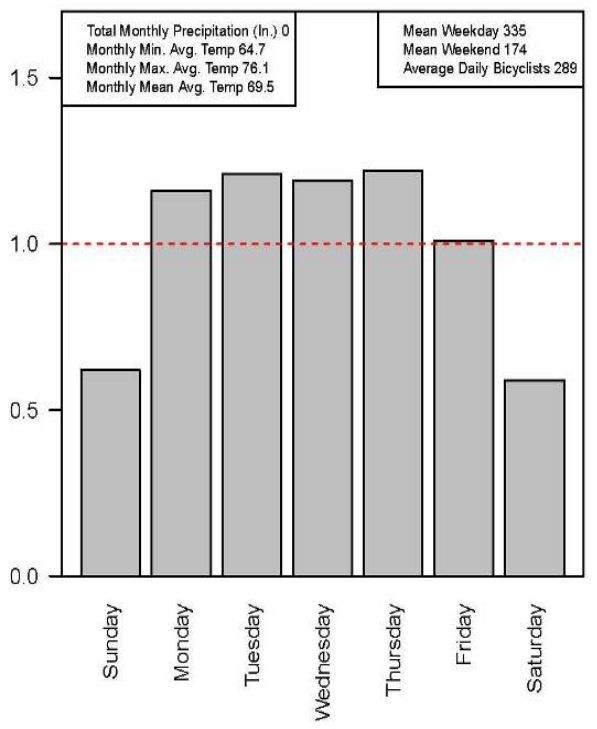


24thEastFilmore Average Daily Factors for September

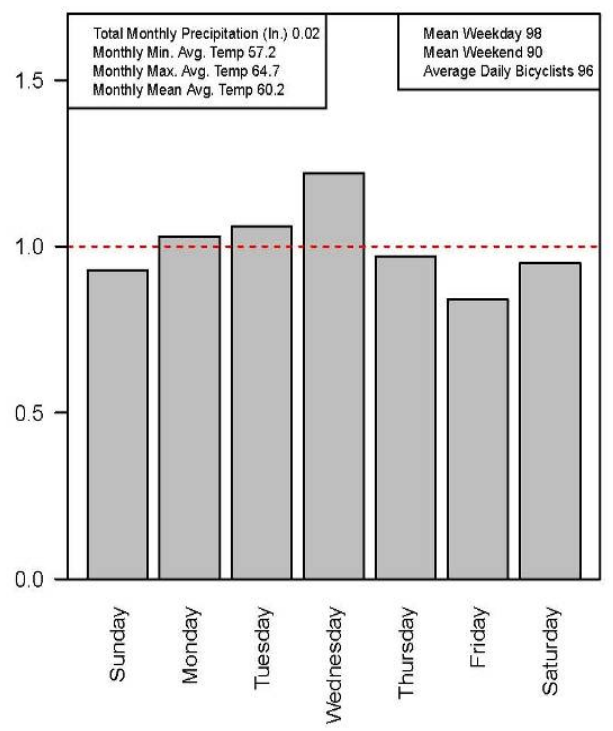

AlderSouth 18th Average Daily Factors for August

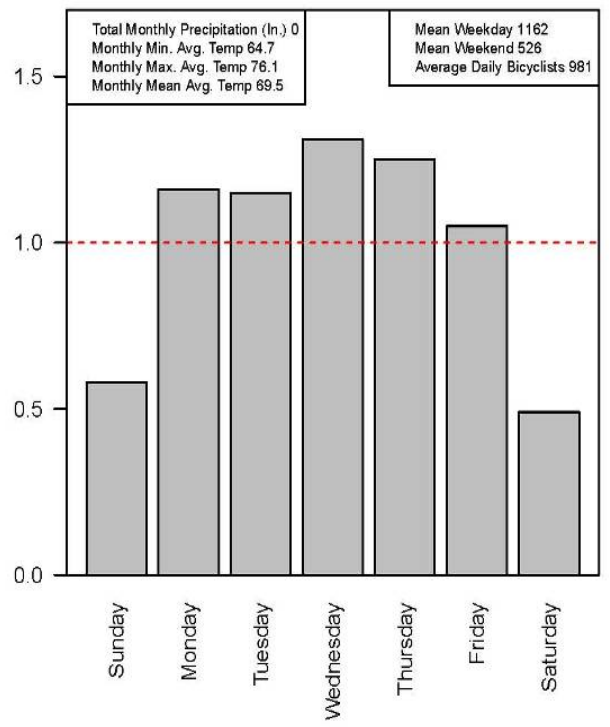

24thWestAdams Average Daily Factors for September

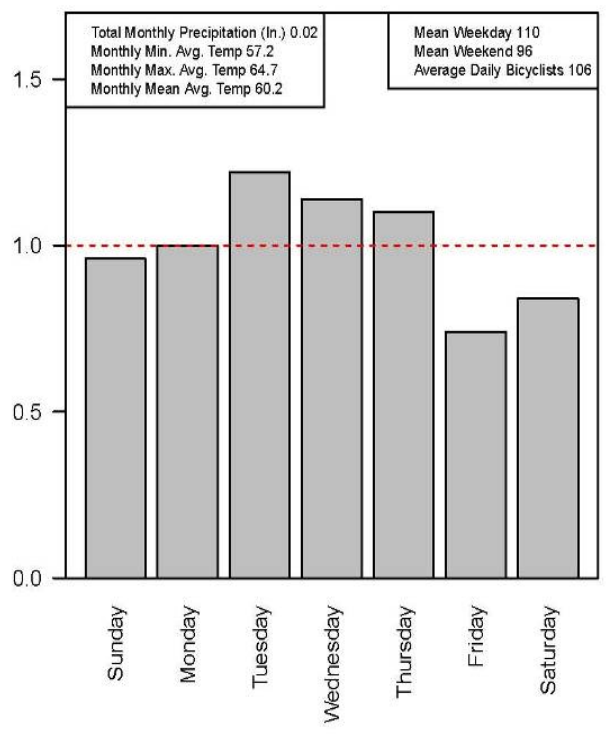

AlderSouthFranklin Average Daily Factors for July

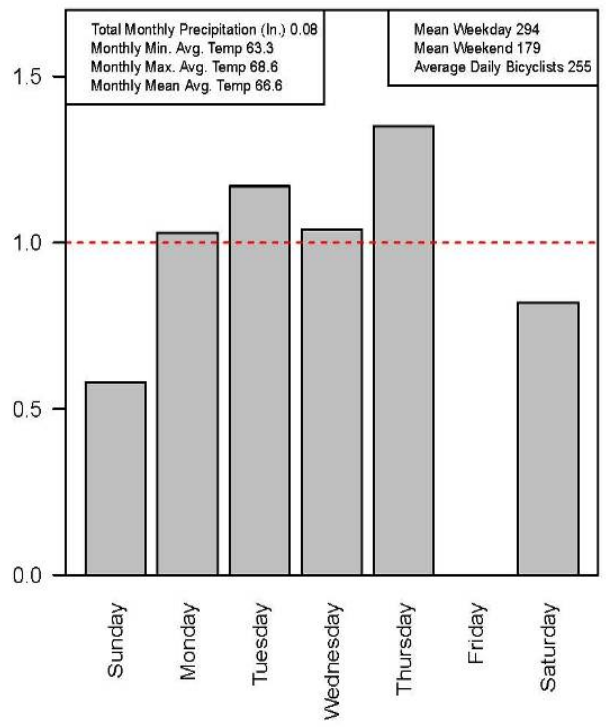




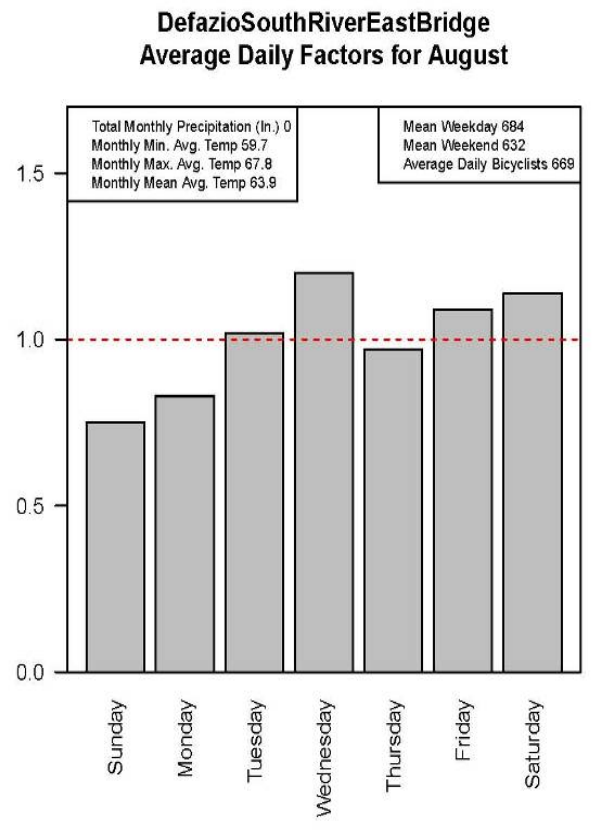

HarrisSouth18th Average Daily Factors for August

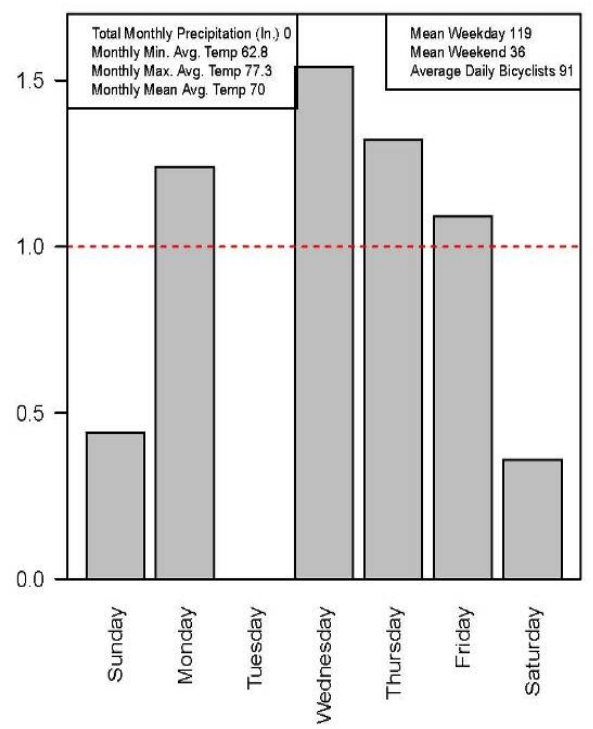

FrohnmayerSouthRiver Average Daily Factors for August

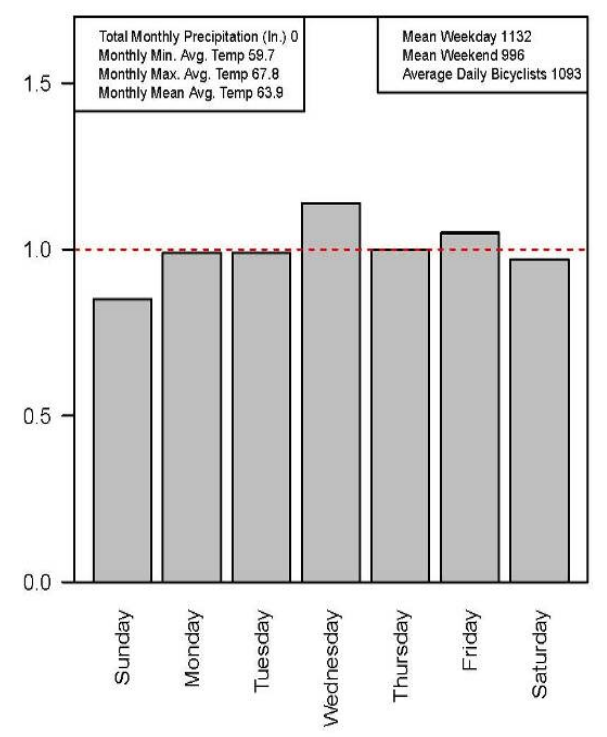

\section{Average Daily Factors for September}

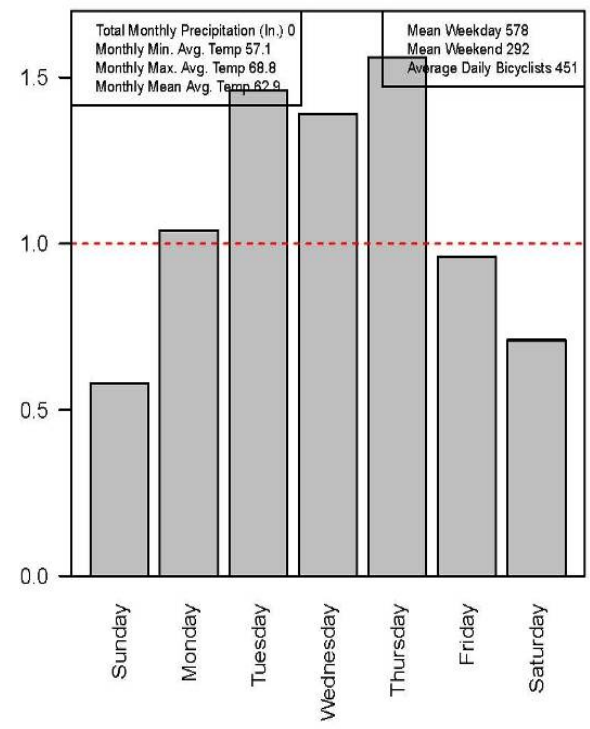


PolkNorth24th

Average Daily Factors for September

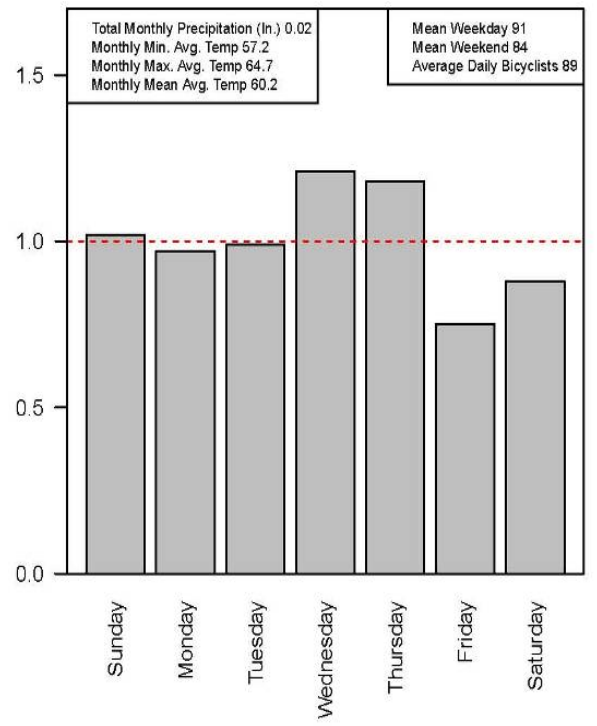

UniversitySouth18th Average Daily Factors for August

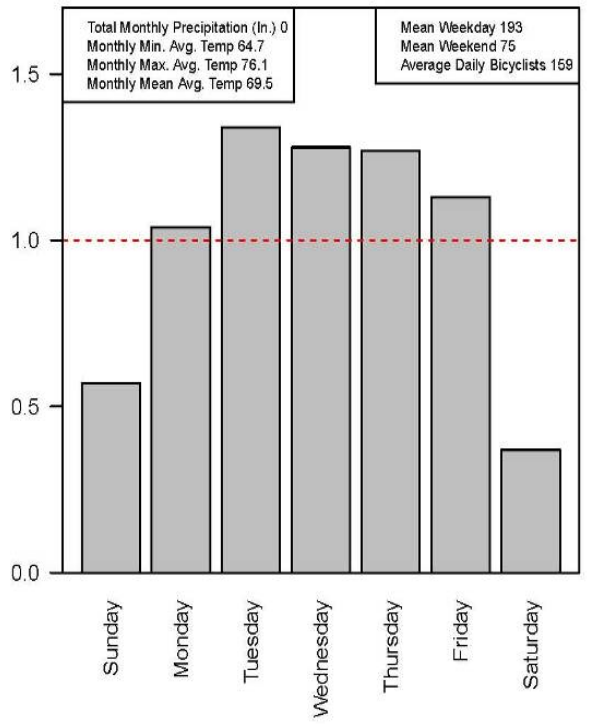


Appendix C

All Daily Counts Data

Download the data in .csv format

All Hourly Counts Data

Download the data in .csv format 


\section{Appendix D}

\section{R Code}

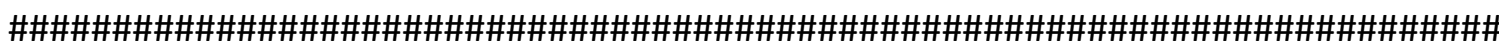
\#\#\#\#\#\#\#\#\#\#\#\#\#\#\#\#\#\#\#\#\#\#\#\#\#\#\#\#\#\#\#\#\#

\# $\quad$ Bicycle Traffic Count Factoring: An Examination of National and Locally Derived Daily \#

\#

Extrapolation Factors Bicycle Traffic Count

\#

\#\#\#\#\#\#\#\#\#\#\#\#\#\#\#\#\#\#\#\#\#\#\#\#\#\#\#\#\#\#\#\#\#\#\#\#\#\#\#\#\#\#\#\#\#\#\#\#\#\#\#\#\#\#\#\#\#\# \#\#\#\#\#\#\#\#\#\#\#\#\#\#\#\#\#\#\#\#\#\#\#\#\#\#\#\#\#\#\#\#\#\#

\#Author : Josh Roll (MAsters of Urban Studies Candidate)

\#Date: 4/12/13

\#Masters Thesis Bicycle Traffic Factoring Analysis

\#.

\#Load and prepare data for analysis

$\#$.

\#Create script defaults

$\#$

\#Create 24-hours vector

Hours. <- as.character(seq $(0,2300,100))$

Hours. <- paste(c(0:23),"00",sep=":")

\#Load Data

$\#$

HourlyPct.. <- read.csv("SummaryTables/HourlyPct.csv", stringsAsFactors =FALSE )

Hourly.. <- read.csv("SummaryTables/Hourly.csv", stringsAsFactors =FALSE )

Daily.. <- read.csv("SummaryTables/Daily.csv",stringsAsFactors =FALSE )

\#Load reference data

CountLocationInformation.. <-

read.csv("SupportingData/CountLocationInformation.csv", as.is =TRUE)

\#Prepare Data

$\#$

\#Rename column headers

colnames(HourlyPct..)[1:24] <- Hours.

colnames(Hourly..)[1:24] <- Hours.

\#Clean out locations and time periods where data is bad or anomalis 
HourlyPct.. <- HourlyPct.. [HourlyPct..\$Location != "AlderSouth18th" ,]

HourlyPct.. <- HourlyPct.. [HourlyPct.. \$Uniqueld != "Total-13thWestAlder-11-282012",]

HourlyPct.. <- HourlyPct.. [HourlyPct.. \$Uniqueld != "Total-13thWestAlder-11-292012",]

HourlyPct.. <- HourlyPct.. [HourlyPct.. \$Uniqueld != "Total-13thWestAlder-11-302012",]

HourlyPct.. <- HourlyPct.. [HourlyPct.. \$Uniqueld != "East-13thWestAlder-11-282012",]

HourlyPct.. <- HourlyPct.. [HourlyPct.. \$Uniqueld != "East-13thWestAlder-11-292012",]

HourlyPct.. <- HourlyPct.. [HourlyPct.. \$Uniqueld != "East-13thWestAlder-11-302012",]

HourlyPct.. <- HourlyPct.. [HourlyPct.. \$Uniqueld != "West-13thWestAlder-11-282012",]

HourlyPct.. <- HourlyPct.. [HourlyPct.. \$Uniqueld != "West-13thWestAlder-11-292012",]

HourlyPct.. <- HourlyPct.. [HourlyPct.. \$Uniqueld != "West-13thWestAlder-11-302012",]

HourlyPct.. <- HourlyPct.. [HourlyPct.. \$Uniqueld != "Total-11.28.2012-11.30.201213thWestAlder",]

HourlyPct.. <- HourlyPct.. [HourlyPct.. \$Location != "13thEastKincaid",]

HourlyPct.. <- HourlyPct.. [HourlyPct.. \$Location != "13thEastLincoln",]

HourlyPct.. <- HourlyPct.. [HourlyPct.. \$Uniqueld != "Total-

DefazioNorthRiverWestBridge-10.03.2012",]

HourlyPct.. <- HourlyPct.. [HourlyPct.. \$Uniqueld != "Total-

DefazioNorthRiverWestBridge-10.04.2012",]

HourlyPct.. <- HourlyPct.. [HourlyPct.. \$Uniqueld != "Total-

DefazioNorthRiverWestBridge-10.05.2012",]

HourlyPct.. <- HourlyPct.. [HourlyPct.. \$Uniqueld != "North-

DefazioNorthRiverWestBridge-10.03.2012",]

HourlyPct.. <- HourlyPct.. [HourlyPct.. \$Uniqueld != "North-

DefazioNorthRiverWestBridge-10.04.2012",]

HourlyPct.. <- HourlyPct.. [HourlyPct.. \$Uniqueld != "North-

DefazioNorthRiverWestBridge-10.05.2012",]

HourlyPct.. <- HourlyPct.. [HourlyPct.. \$Uniqueld != "South-

DefazioNorthRiverWestBridge-10.03.2012",]

HourlyPct.. <- HourlyPct.. [HourlyPct.. \$Uniqueld != "South-

DefazioNorthRiverWestBridge-10.04.2012",]

HourlyPct.. <- HourlyPct.. [HourlyPct.. \$Uniqueld != "South-

DefazioNorthRiverWestBridge-10.05.2012",]

Hourly.. <- Hourly.. [Hourly.. \$Location != "AlderSouth18th",] 
Hourly.. <- Hourly.. [Hourly.. \$Uniqueld != "Total-13thWestAlder-11-28-2012",] Hourly.. <- Hourly.. [Hourly.. \$Uniqueld != "Total-13thWestAlder-11-29-2012",] Hourly.. <- Hourly.. [Hourly.. \$Uniqueld != "Total-13thWestAlder-11-30-2012",] Hourly.. <- Hourly.. [Hourly.. \$Uniqueld != "East-13thWestAlder-11-28-2012",] Hourly.. <- Hourly.. [Hourly.. \$Uniqueld != "East-13thWestAlder-11-29-2012",] Hourly.. <- Hourly.. [Hourly.. \$Uniqueld != "East-13thWestAlder-11-30-2012",] Hourly.. <- Hourly.. [Hourly.. \$Uniqueld != "West-13thWestAlder-11-28-2012",] Hourly.. <- Hourly.. [Hourly.. \$Uniqueld != "West-13thWestAlder-11-29-2012",] Hourly.. <- Hourly.. [Hourly.. \$Uniqueld != "West-13thWestAlder-11-30-2012",] Hourly.. <- Hourly.. [Hourly.. \$Location != "13thEastKincaid",] Hourly.. <- Hourly.. [Hourly.. \$Location != "13thEastLincoln",] Hourly.. <- Hourly.. [Hourly.. \$Uniqueld != "Total-DefazioNorthRiverWestBridge10.03.2012",]

Hourly.. <- Hourly.. [Hourly.. \$Uniqueld != "Total-DefazioNorthRiverWestBridge10.04.2012",]

Hourly.. <- Hourly.. [Hourly.. \$Uniqueld != "Total-DefazioNorthRiverWestBridge10.05.2012",]

Hourly.. <- Hourly.. [Hourly.. \$Uniqueld != "North-DefazioNorthRiverWestBridge10.03.2012",]

Hourly.. <- Hourly.. [Hourly.. \$Uniqueld != "North-DefazioNorthRiverWestBridge10.04.2012",]

Hourly.. <- Hourly.. [Hourly.. \$Uniqueld != "North-DefazioNorthRiverWestBridge10.05.2012",]

Hourly.. <- Hourly.. [Hourly.. \$Uniqueld != "South-DefazioNorthRiverWestBridge10.03.2012",]

Hourly.. <- Hourly.. [Hourly.. \$Uniqueld != "South-DefazioNorthRiverWestBridge10.04.2012",]

Hourly.. <- Hourly.. [Hourly.. \$Uniqueld != "South-DefazioNorthRiverWestBridge10.05.2012",]

Hourly.. <- Hourly..[Hourly..\$Location != "AgateSouth18thSB" \&\& Hourly..\$Period != "08.22.2012-08.30.2012",]

Hourly.. <- Hourly..[Hourly..\$Location != "AgateSouth18thNB" \&\& Hourly..\$Period != "08.22.2012-08.30.2012",]

\#Clean out all data not having 24 hours worth of data Hourly.. <- Hourly..[Hourly..\$ObsHours == 24,] HourlyPct.. <- HourlyPct..[HourlyPct..\$ObsHours ==24,] \#Clean out data without an observed daily total Hourly.. <- Hourly..[Hourly..\$DailyCounts >0,] HourlyPct.. <- HourlyPct..[HourlyPct..\$DailyCounts >0,]

\#Remove Tuesday Thursday 
Hourly.. <- Hourly..[Hourly..\$Weekday\%in\%c("Tuesday","Thursday"),]

HourlyPct.. <- HourlyPct..[HourlyPct..\$Weekday\%in\%c("Tuesday","Thursday"),]

\#Add Factor Groups

Hourly..\$FactorGroup <-

CountLocationInformation..\$FactorGroup[match(Hourly..\$Location,

CountLocationInformation..\$Location)]

HourlyPct..\$FactorGroup <-

CountLocationInformation..\$FactorGroup[match(HourlyPct..\$Location,

CountLocationInformation..\$Location)]

$\#$......

\#Scenario 1 Application of Factors

\#

\#Load saved factor values

load(file $=$

"T:/Data/COUNTS/BicycleCounts/LCOG/ExtrapolationValidationProject/Data/Factors_.R Data")

\#Specify season to analyze

SeasonToAnalyze <- "Fall"

Factors_[["NoFacility"]] <- Factors_[["Local"]]

Result_<- list()

Result2_<- list()

Labels_<- list()

AmPeak_<- list()

PmPeak_<- list()

\#lterate through each time period

for(period in c("Am","Pm"))\{

TempHourly.. <- Hourly..

\#Create lists to store results

Result_<- list()

Result2_<- list() 


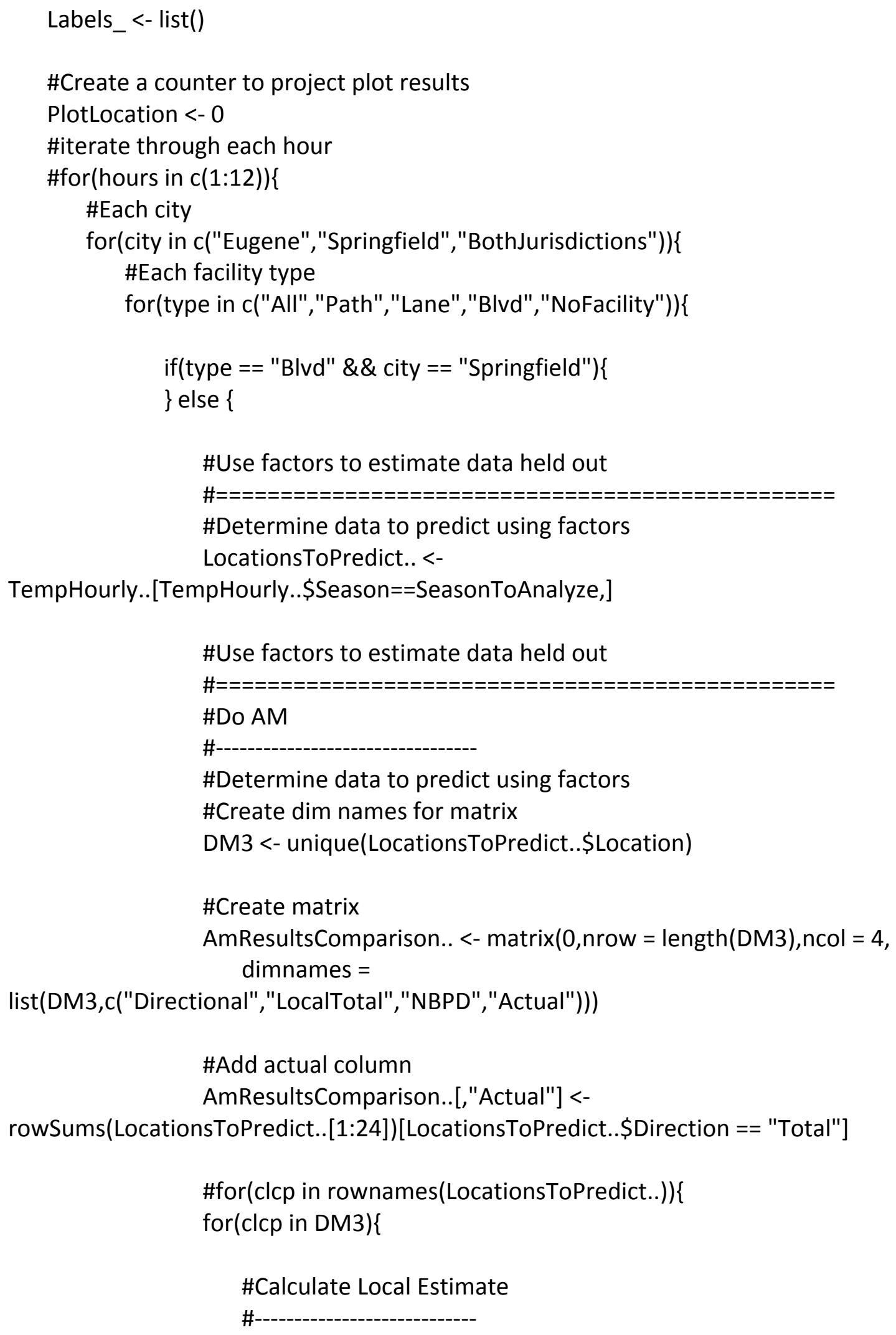


\#Grab data for direction and location

DataTotal. <- LocationsToPredict..[LocationsToPredict..\$Direction

$==$ "Total" \& LocationsToPredict..\$Location == clcp,][1:24]

\#2 hours

AmPeakHours. $<-8: 9$

\#Determine is location is oneway

IsOneway <-

CountLocationInformation..\$IsOneway[CountLocationInformation..\$Location\%in\%clcp]

\#Do if data exists

if(length(rownames(DataTotal. )) != 0) \{

\#Determine PM "sample"

AmSample. <- DataTotal.[AmPeakHours.]

\#Grab location

Location <- unlist(strsplit(clcp,split="-"))[4]

Location <- clcp

\#To estimate using Total factors

\#+++++++++++++++++++++++++++++++++

\#DirectionalAverage. <- FinalTotalAvg.

DirectionalAverage. <- Factors_[[type]][["Total"]]

\#Determine aggregate AM percentage

AMPercentage.dr <- sum(DirectionalAverage.[AmPeakHours.])

\#Calculate value of single percentage $1 \%=x$ )

Xpct <- sum(AmSample.) / (AMPercentage.dr * 100)

\#Multiply by directional average percentages to estimate

"sample" data

LocalSum <- round(sum( Xpct * 100),0)

\#Store Local results

AmResultsComparison..[clcp,"LocalTotal"] <- LocalSum

\#To estimate using Inbound factors

\#+++++++++++++++++++++++++++++++++++

\#Determine Inbound direction

InboundDirection <-

CountLocationInformation..\$Inbound[CountLocationInformation..\$Location\%in\%clcp]

\#Assign oneway

names(InboundDirection) <-

CountLocationInformation..\$OnewayDirection[CountLocationInformation..\$Location\%in

$\%$ clcp]

\#Select inbound data 
Datalnbound. <-

LocationsToPredict..[LocationsToPredict..\$Inbound == TRUE \&

LocationsToPredict..\$Location == clcp,][1:24]

DirectionalAverage.<- Factors_[[type]][["Inbound"]]

AmSample. <- sum(Datalnbound.[AmPeakHours.] )

\#Determine aggregate APM percentage

AMPercentage.dr <- sum(DirectionalAverage.[AmPeakHours.])

"sample" data

\#Multiply by directional average percentages to estimate

InboundSum <- round(AmSample. / AMPercentage.dr,0)

\#To estimate using Outbound factors

\#+++++++++++++++++++++++++++++++++

\#Determine Inbound direction

OutboundDirection <-

CountLocationInformation..\$Outbound[CountLocationInformation..\$Location\%in\%clcp]

\#Assign oneway

names(OutboundDirection) <-

CountLocationInformation..\$OnewayDirection[CountLocationInformation..\$Location\%in \%clcp]

\#Select inbound data

DataOutbound. <-

LocationsToPredict..[LocationsToPredict..\$Outbound == TRUE \&

LocationsToPredict..\$Location == clcp,][1:24]

DirectionalAverage. <- Factors_[[type]][["Outbound"]]

AmSample. <- sum(DataOutbound.[AmPeakHours.] )

\#Determine aggregate AM percentage

AMPercentage.dr <- sum(DirectionalAverage.[AmPeakHours.])

"sample" data

\#Multiply by directional average percentages to estimate

OutboundSum <- round(AmSample. / AMPercentage.dr,0)

\#Store Directional Results

\#Consider if location is oneway - only count one direction

if(IsOneway)\{

if $($ InboundDirection $==$ names(InboundDirection $))\{$

AmResultsComparison..[clcp,"Directional"] <-

InboundSum

\}

if(OutboundDirection $==$ names(OutboundDirection $))\{$ 
OutboundSum

+ OutboundSum

"sample" data

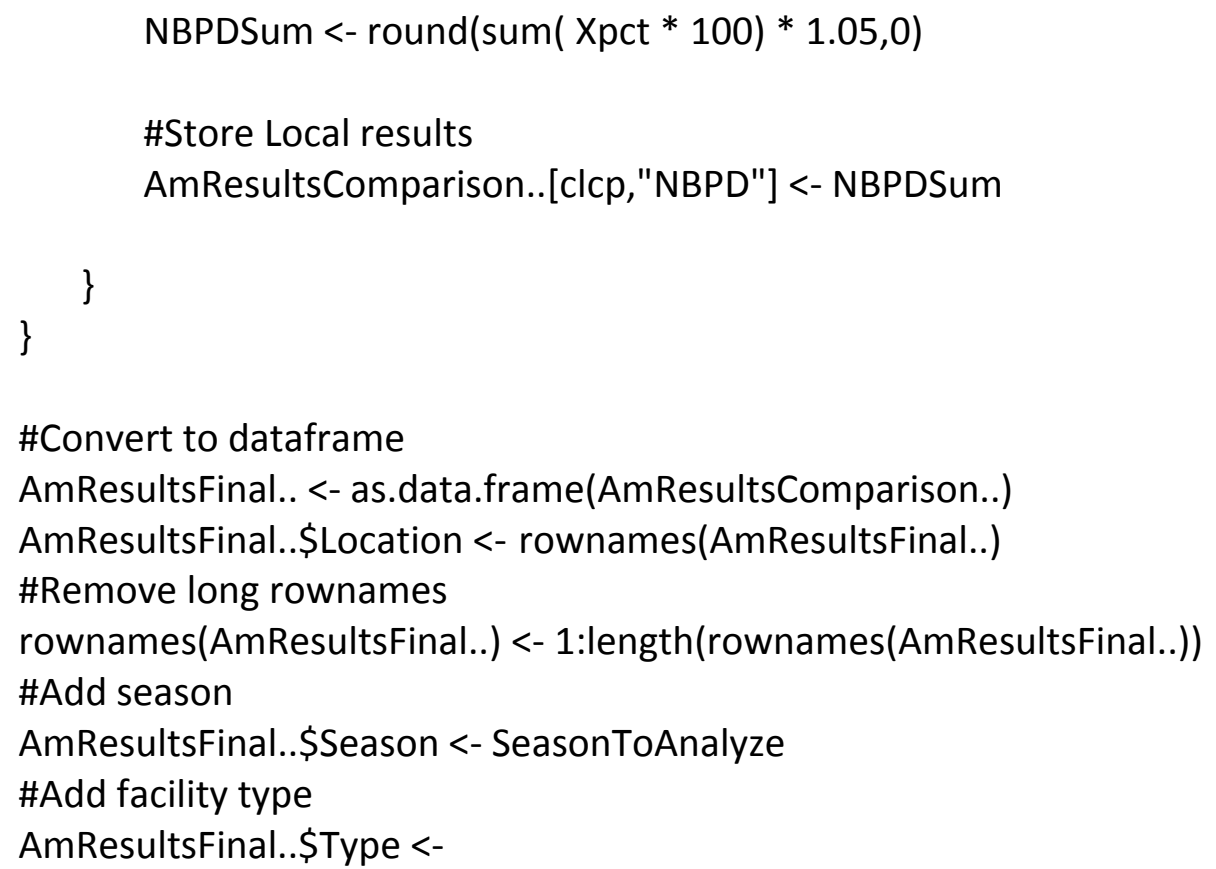

\#Use factors to estimate data held out

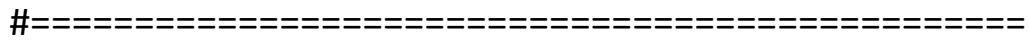


\#Do PM

\#-

\#Determine data to predict using factors

\#Create dim names for matrix

DM3 <- unique(LocationsToPredict..\$Location)

\#Create matrix

PmResultsComparison. . <- matrix(0,nrow = length(DM3), ncol = 4, dimnames $=$

list(DM3,c("Directional","LocalTotal","NBPD","Actual")))

\#Add actual column

PmResultsComparison..[,"Actual"] <-

rowSums(LocationsToPredict..[1:24])[LocationsToPredict..\$Direction == "Total"]

\#for(clcp in rownames(LocationsToPredict..))\{

for(clcp in DM3)\{

\#Calculate Local Estimate

\#-

\#Grab data for direction and location

DataTotal. <- LocationsToPredict..[LocationsToPredict..\$Direction

$==$ "Total" \& LocationsToPredict..\$Location == clcp,][1:24]

\#2 hours

PmPeakHours. <- 17:18

\#Determine is location is oneway

IsOneway <-

CountLocationInformation..\$IsOneway[CountLocationInformation..\$Location\%in\%clcp]

\#Do if data exists

if(length(rownames(DataTotal. )) != 0) \{

\#Determine PM "sample"

PmSample. <- DataTotal.[PmPeakHours.]

\#Grab location

Location <- clcp

\#To estimate using Total factors

\#+++++++++++++++++++++++++++++++++++

\#DirectionalAverage. <- FinalTotalAvg.

DirectionalAverage. <- Factors_[[type]][["Total"]]

\#Determine aggregate AM percentage

PMPercentage.dr <- sum(DirectionalAverage.[PmPeakHours.]) 


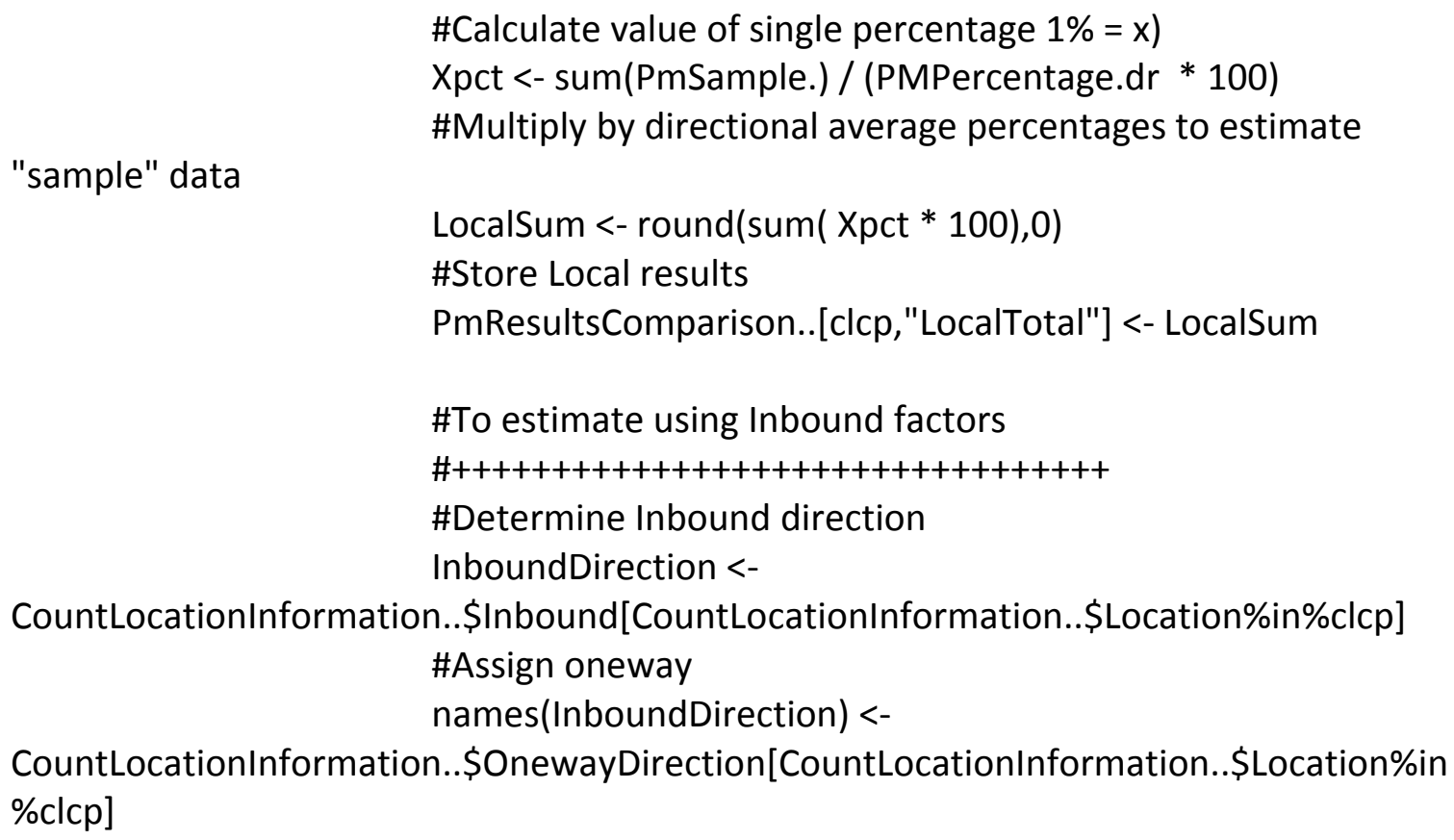

\#Select outbound data 
DataOutbound. <-

LocationsToPredict..[LocationsToPredict..\$Outbound == TRUE \&

LocationsToPredict..\$Location == clcp,][1:24]

DirectionalAverage. <- Factors_[[type]][["Outbound"]]

PmSample. <- sum(DataOutbound.[PmPeakHours.] )

\#Determine aggregate AM percentage

PMPercentage.dr <- sum(DirectionalAverage.[PmPeakHours.])

"sample" data

\#Multiply by directional average percentages to estimate

OutboundSum <- round(PmSample. / PMPercentage.dr,0)

InboundSum

\#Store Directional Results

\#Consider if location is oneway - only count one direction

if(IsOneway)\{

if $($ InboundDirection $==$ names $($ InboundDirection $))\{$

PmResultsComparison..[clcp,"Directional"] <-

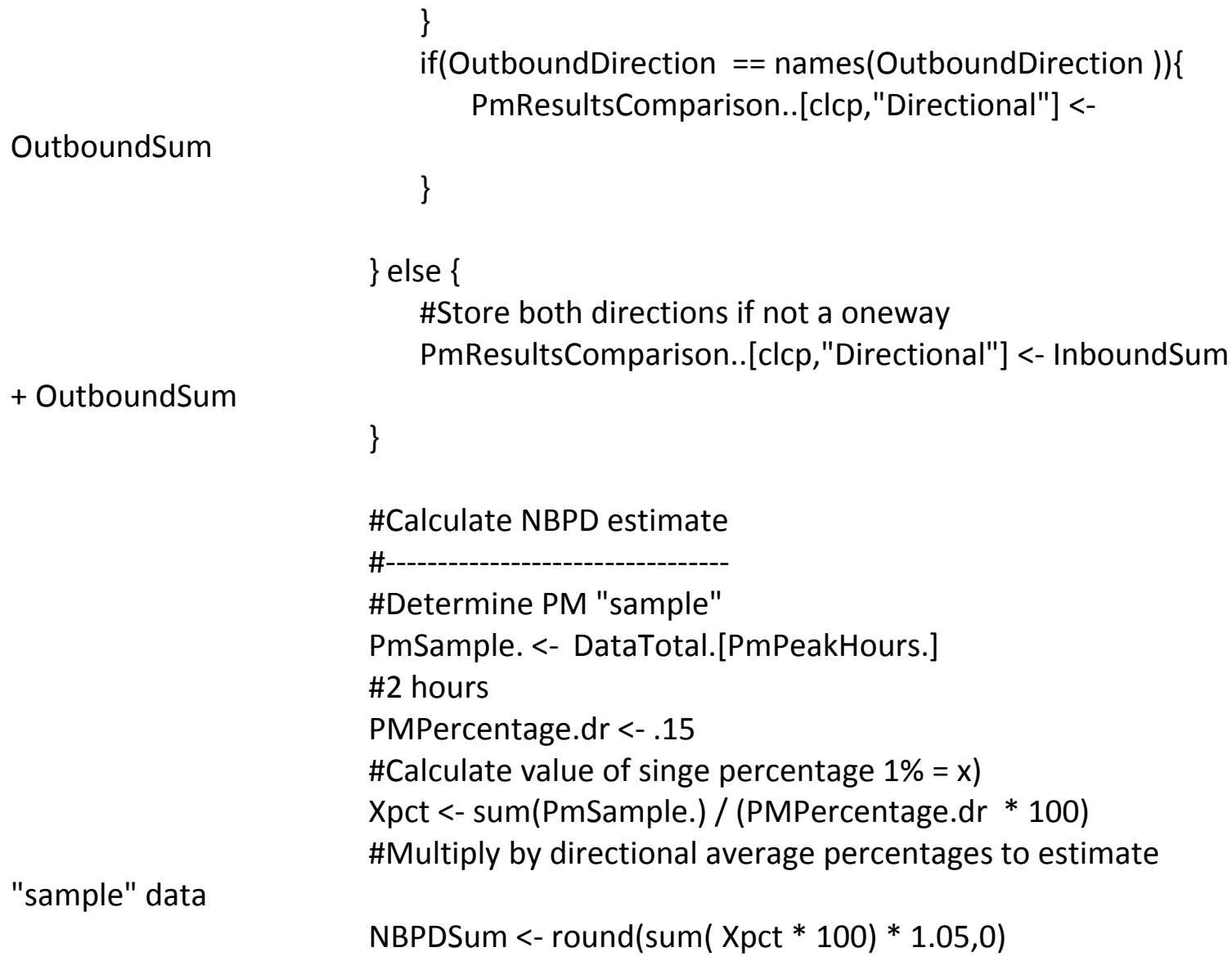

OutboundSum

+ OutboundSum 


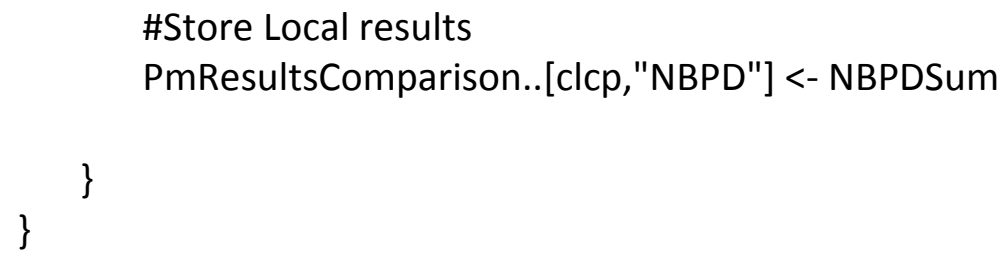

\#Convert to dataframe

PmResultsFinal.. <- as.data.frame(PmResultsComparison..)

PmResultsFinal..\$Location <- rownames(PmResultsFinal..)

\#Remove long rownames

rownames(PmResultsFinal..) <- 1:length(rownames(PmResultsFinal..))

\#Add season

PmResultsFinal..\$Season <- SeasonToAnalyze

\#Add facility type

PmResultsFinal..\$Type <-

CountLocationInformation..\$Type[match(PmResultsFinal..\$Location,CountLocationInfor mation..\$Location)]

\#Select locations to check results

\#Never counted in Estimation data

if(city == "Eugene")\{

ToPlot. <-

c("15thWestJefferson","AlderNorth27th","AmazonPathNorth24th",

"DefazioSouthRiverEastBridge","DeltaBPBridgeEastGoodPasture","FernRidgeEastDaneb o","FernRidgeEastSeneca", "FernRidgeSouthWestMoeBr",

"FirEastRiverRd"

,"FriendlySouth18th","HighNorth13th","HighSouth4th","MonroeSouth8th",

"NorthbankSouthGreenwayBr",

"NorthbankSouthOwossaBr","NorthbankWestDeltaHwy/","NorthbankEastKnickerbocker ",

"PearlSouth19th",

"SouthbankSouthGreenwayBr","UniversityNorth24th/", "WillametteNorth15th")

\}

if(city == "Springfield")\{

\#Springfield

ToPlot. <-

c("32ndSouthOregon", "EWEBPathEast5th", "EWEBPathEastPioneerPkwy","DEastPioneer PkwyPath", 
"GWestMohawk","GoodpastureEastDeltaHwy","GatewayBPBridgeEastl5","Gateway StreetEastl5" ,"HarlowEastl5","PioneerPkwySouthQ","ThurstonEast58th")

\}

if(city == "BothJurisdictions")\{

ToPlot. <-

c("15thWestJefferson","AlderNorth27th","AmazonPathNorth24th",

"DefazioSouthRiverEastBridge","DeltaBPBridgeEastGoodPasture","FernRidgeEastDaneb o","RichardsonBridge","HeronBridgeSouthFernRidge",

"FirEastRiverRd"

,"FriendlySouth18th","GoodpastureEastDeltaHwy","GWestMohawk","HighNorth13th"," HighSouth4th","MonroeSouth8th",

"NorthbankSouthGreenwayBr",

"EastbankSouthOwossoBr","NorthbankWestDeltaHwy","NorthbankEastKnickerbocker", "PearlSouth19th",

"SouthbankSouthGreenwayBr","UniversityNorth24th", "WillametteNorth15th",

"32ndSouthOregon","EWEBPathEast5th","EWEBPathEastPioneerPkwy","DEastPioneerP kwyPath",

"GatewayBPBridgeEast15","GatewayStreetEastl5"

,"HarlowEastI5","PioneerPkwySouthQ","ThurstonEast58th")

\}

\#Select location of interest

AmResultsFinalPlot.. <-

AmResultsFinal..[AmResultsFinal..\$Location\%in\%ToPlot.,]

PmResultsFinalPlot.. <-

PmResultsFinal..[PmResultsFinal..\$Location\%in\%ToPlot.,]

\#Select facility type

if(type != "All")\{

AmResultsFinalPlot.. <-

AmResultsFinalPlot..[AmResultsFinalPlot..\$Type == type,]

PmResultsFinalPlot.. <-

PmResultsFinalPlot..[PmResultsFinalPlot..\$Type == type,]

\}

\# \#Create table for output

\#----------------------------- 
AmResultsFinalPlot..\$Period <- "AM"

PmResultsFinalPlot..\$Period <- "PM"

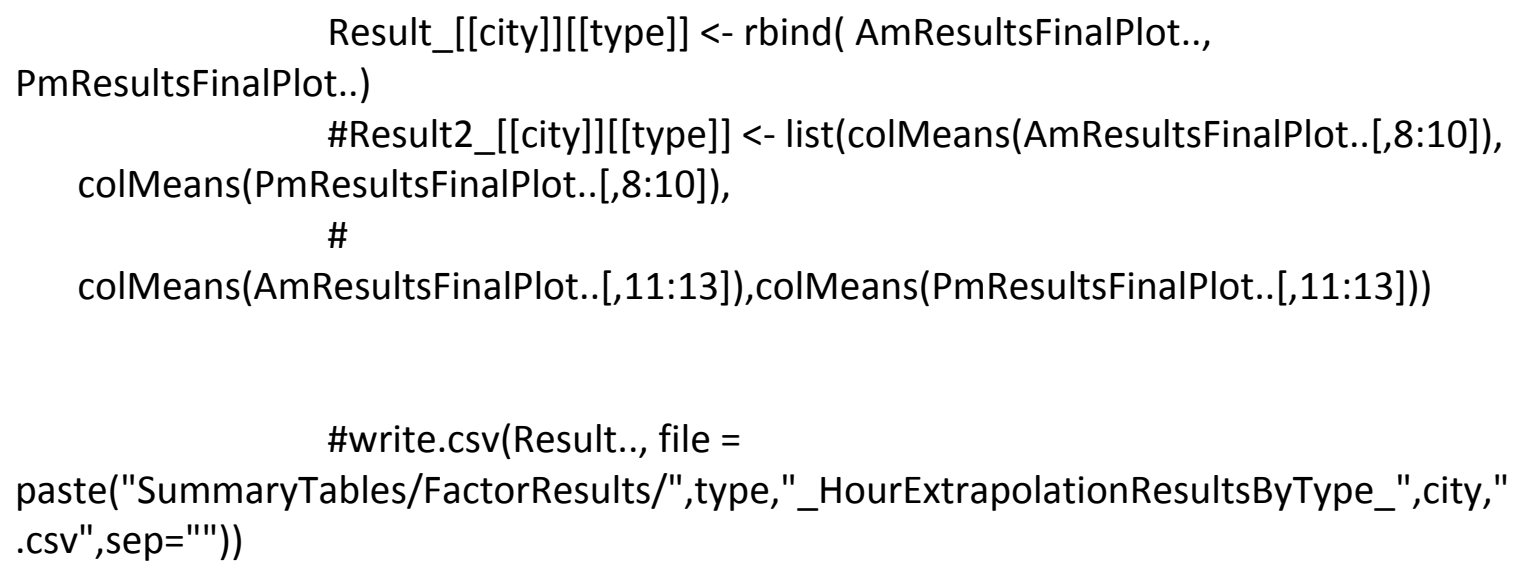


\#Create data frame

TempResults.. <- as.data.frame(Result_[["BothJurisdictions"]][[type]])

if(nrow(TempResults..) $>0)\{$

\#Select only columns of interest

TempResults.. <-

TempResults..[,c("LocalTotal","Directional","NBPD","Actual","Location","Period","Type") ]

\#Add city

\#TempResults..\$City <- cities

\#Add to master results dataframe

ResultsFormatted.. <- rbind(ResultsFormatted..,TempResults..)

\}

\}

\#Change row names

rownames(ResultsFormatted..) <- 1:nrow(ResultsFormatted..)

\#Reformat factors for display

FinalFactors_<- list()

for(period in c("AM","PM"))\{

Am. $<-$ NULL

Pm. $<-$ NULL

Period. <- NULL

for(type in c("Path","Lane","Blvd","NoFacility"))\{

if(period == "AM") \{

Period <-round(sum(Factors_[[type]][["Total"]][8:9]),2)

names(Period) <- type

Period. <- c(Period.,Period)

\}

if(period $==$ "PM") \{

Period <- round(sum(Factors_[[type]][["Total"]][17:18]),2)

names(Period) <- type

Period. <- c(Period.,Period)

\}

\} 


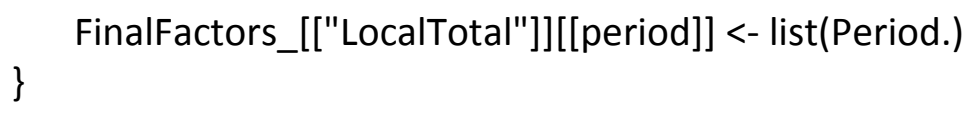


\#Determine symbol for plot

\#BikeFac <- CountLocationInformation..\$FactorGroup[match(tl,

CountLocationInformation..\$Location)]

BikeFac <- CountLocationInformation..\$Type[match(tl,

CountLocationInformation..\$Location)]

if $($ BikeFac $==$ "Path") $\{$ Pch $<-5\}$

if(BikeFac == "Lane") $\{$ Pch $<-10\}$

if(BikeFac $==$ "Blvd") $\{$ Pch $<-18\}$

if(BikeFac $==$ "NoFacility") $\{$ Pch $<-8\}$

\#Plot is available

if(!(is.na(Obs)))\{

plot(Obs Est, main = paste("Results for",result, period, sep=" "),xlim =

Xlim, ylim = Ylim, pch = Pch, col = "black")

$\operatorname{par}($ new $=$ TRUE $)$

\#Store

Obs_[[t 1$]]<-$ Obs

Est_[[tl]]<- Est

\}

\}

$\operatorname{par}(\mathrm{xpd}=\mathrm{FALSE})$

\#Add 45 reference line

abline $(a=0, b=1, c o l=$ "red")

$\operatorname{par}(\mathrm{xpd}=\mathrm{TRUE})$

\#Develop summary

Location. <- as.character(do.call("rbind",strsplit(names(Obs_),"-"))[,1])

\#Season <- as.character(do.call("rbind",strsplit(names(Obs_),"-"))[,2])

BestResult.. <- data.frame(Location = Location., Obs = unlist(Obs_F,F), Est =

unlist(Est_,F,F))

\#BestResult..\$PctDiff <- round(BestResult..\$Obs / BestResult..\$Est,2)

\#BestResult..\$AbsPctDiff <- round( abs(1-(BestResult..\$Obs / BestResult..\$Est)),2)

BestResult..\$PctDiff <- round(BestResult..\$Est / BestResult..\$Obs,2)

BestResult..\$AbsPctDiff <- round( abs(1-(BestResult..\$Est / BestResult..\$Obs)),2)

BestResult..\$Type <-

CountLocationInformation..\$Type[match(BestResult..\$Location,

CountLocation Information..\$Location)]

BestResult..\$FedClass <-

CountLocationInformation..\$FedClass[match(BestResult..\$Location,

CountLocationInformation..\$Location)] 
BestResult..\$FactorGroup <-

CountLocationInformation..\$FactorGroup[match(BestResult..\$Location, CountLocationInformation..\$Location)]

BestResult..\$Locationld <-

CountLocationInformation..\$Locationld[match(BestResult..\$Location,

CountLocationInformation..\$Location)]

BestResult.. <- BestResult..[order(BestResult..\$AbsPctDiff),]

\#BestResult.. <- BestResult..[order(BestResult..\$Type),]

\#Store final results data frame

FinalResults_[[paste(result, period, sep="-")]] <- BestResult..

\#Add legend

RMSE <- round(summary(Im(unlist(Obs_) unlist(Est_)))\$sigma,2)

RMSE <- paste("RMSE = ",RMSE,sep="")

RSquared <- round(summary(Im(unlist(Obs_) unlist(Est_)))\$r.squared,2)

RSquared <- paste("R-squared = ", RSquared, sep= "")

MeanAbsDiff <- round(mean(BestResult..\$AbsPctDiff),2)

MeanAbsDiff <- paste("Mean Abs Diff = ", MeanAbsDiff)

\#MeanAbsDiff.Fg <-

round(tapply(BestResult..\$AbsPctDiff,BestResult..\$FactorGroup,mean),2)

MeanAbsDiff.Fg <-

round(tapply(BestResult..\$AbsPctDiff,BestResult..\$Type,mean),2)

\#Add legend 1

legend("topleft",title = "Results Summary", legend =c(RMSE, RSquared, MeanAbsDiff) )

\#Add legend 2

legend(3150,3100,title = "Mean Abs Diff by Factor Group", legend $=c$ (paste(names(MeanAbsDiff.Fg[1]),MeanAbsDiff.Fg[1],sep="-"), paste(names(MeanAbsDiff.Fg[2]),MeanAbsDiff.Fg[2],sep=""),paste(names(MeanAbsDiff.Fg[3]),MeanAbsDiff.Fg[3],sep="-"), paste(names(MeanAbsDiff.Fg[4]),MeanAbsDiff.Fg[4],sep="-")),cex = .9)

\#Add legend 3

Factors. <- unlist(FinalFactors_[[result]][[period]])

Factors. <- paste(names(Factors.),Factors.,sep="-")

\#legend("bottomright", legend = c("Factors","Local",AmTotalFactors.))

legend $(3150,2000$, legend $=c($ "Factors", result,Factors.))

\#Add legend 4 


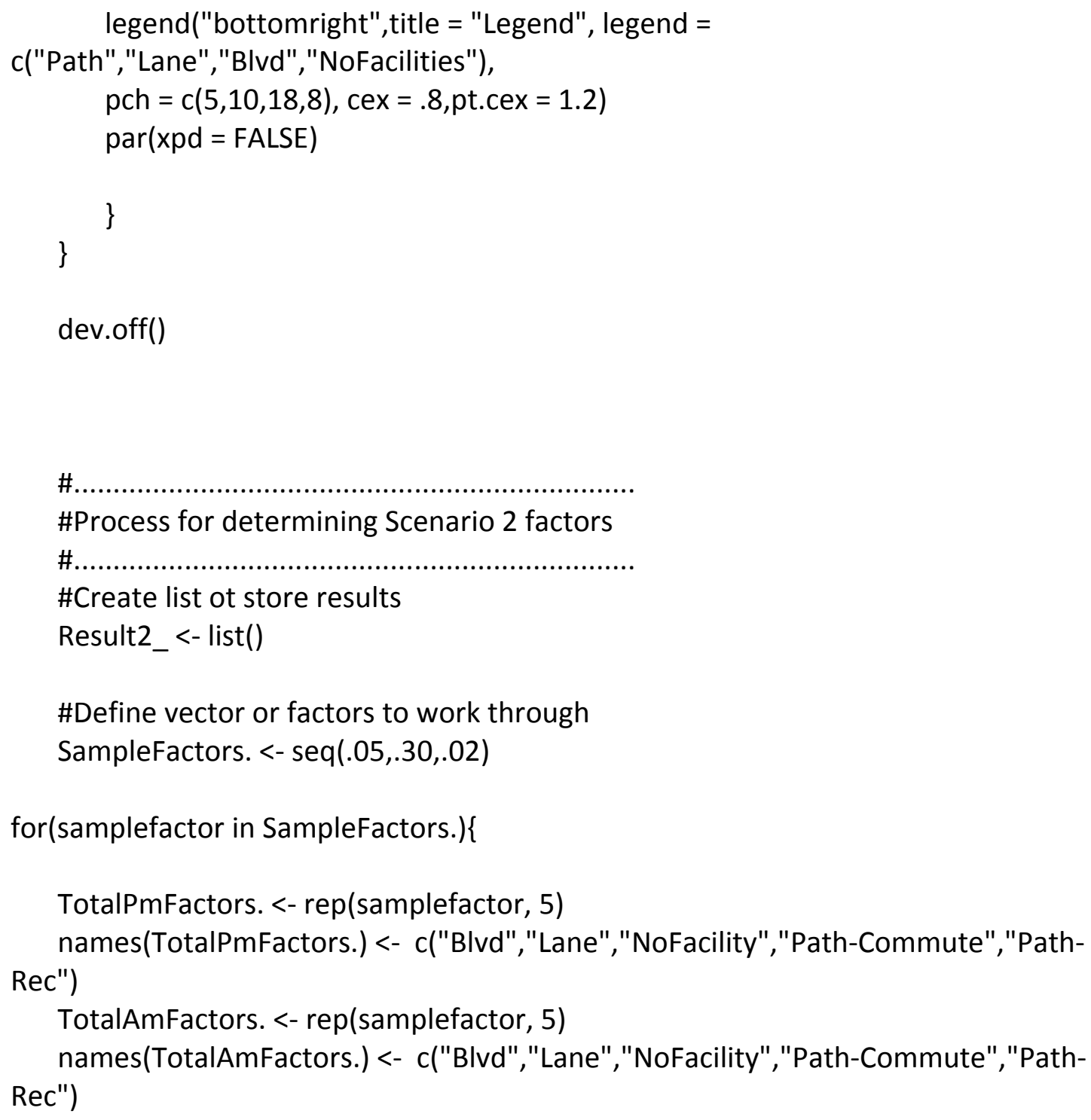

\#Apply Factors to count stations not selected for factor creation

$\#$.......

\#Determine count stations to apply factors too

TestData.. <- Hourly..

\#Create vector of test locations

TestLocations. <- unique(TestData..\$Location)

\#Remove All days but Tuesday and Thursday

TestData.. <- TestData..[TestData..\$Weekday\%in\%c("Tuesday","Thursday"),]

\#Remove Spring

TestData.. <- TestData..[TestData..\$Season != "Spring",]

\#Append factor Groups 
TestData..\$FactorGroup <-

CountLocationInformation..\$FactorGroup[match(TestData..\$Location,

CountLocationInformation..\$Location)]

\#Create an observed array

\#--

\#Create an array to store observed counts

ObservedCounts..DayClSe <- array(NA, c(length(TestLocations.), 3, 2), dimnames = list(TestLocations., c("Summer","Fall","Winter"),

c("Tuesday","Thursday")))

\#Populate array with observed values

for(tl in TestLocations.) \{

\#Select data

TempTestData.. <- TestData..[TestData..\$Location == tl,]

\#Do Total

\#+++++++++++++++++++++++++++++++

TempTestData.. <- TempTestData..[TempTestData..\$Direction == "Total",]

\#Do each day

for(day in TempTestData..\$Date)\{

\#Establish day

Weekday <- TempTestData..\$Weekday[TempTestData..\$Date\%in\%day]

\#Establish season

season <- TempTestData..\$Season[TempTestData..\$Date\%in\%day]

\#Do Am

ObservedCount <-

sum(TempTestData..[TempTestData..\$Date\%in\%day,][1:24])

\#Store result

ObservedCounts..DayClSe [tl,season,Weekday] <- ObservedCount

\}

\}

\#Estimate using factors

\#-------------------------------------

\#Create Array to store raw results

\#Total

AmTotalResults..DayClSe <- $\operatorname{array}(\mathrm{NA}, \mathrm{c}($ length(TestLocations.), 3, 2), dimnames = list(TestLocations., c("Summer","Fall","Winter"),

c("Tuesday","Thursday")))

PmTotalResults..DayCISe <- $\operatorname{array}(\mathrm{NA}, \mathrm{c}($ length(TestLocations.), 3, 2), dimnames = list(TestLocations., c("Summer","Fall","Winter"), 


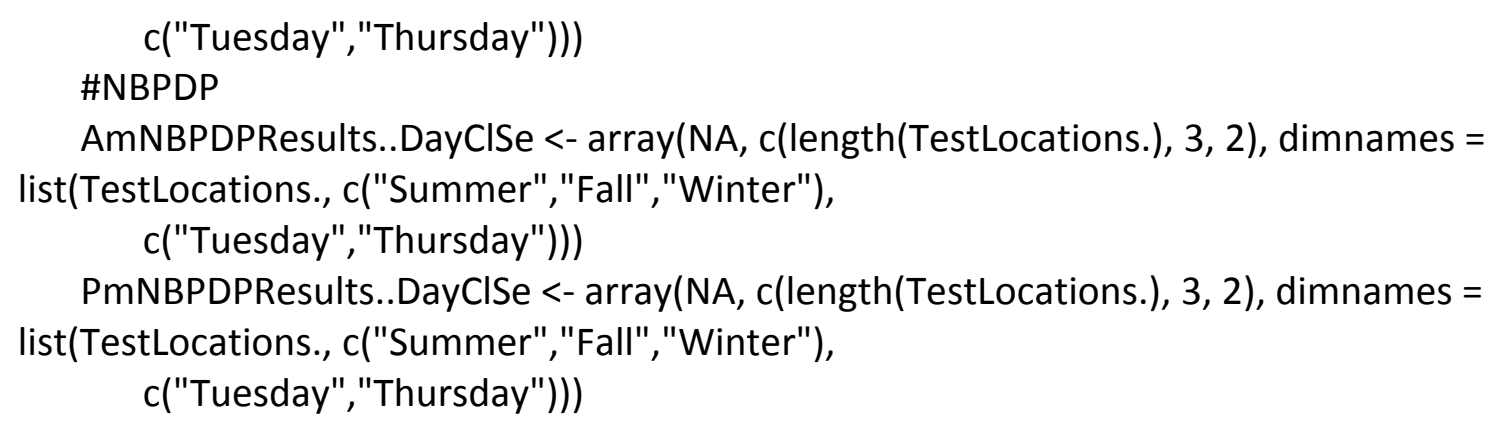

AmNBPDPResults..DayClSe <- array(NA, c(length(TestLocations.), 3, 2), dimnames = list(TestLocations., c("Summer","Fall","Winter"),

c("Tuesday","Thursday")))

PmNBPDPResults..DayClSe <- array(NA, c(length(TestLocations.), 3, 2), dimnames = list(TestLocations., c("Summer","Fall","Winter"),

c("Tuesday","Thursday")))

\section{\#Apply Factors}

\#......

\#Total

for(tl in TestLocations.) \{

\#Select data

TempTestData.. <- TestData..[TestData..\$Location == tl,]

\#Establish facility type

\#type <- unique(TempTestData..\$Type)

type <- unique(TempTestData..\$FactorGroup)

\#Do Total

\#+++++++++++++++++++++++++++++++

TempTestData.. <- TempTestData..[TempTestData..\$Direction == "Total",]

\#Do each day

for(day in TempTestData..\$Date)\{

\#Local Total

\#+++++++++++++++++++++++++++++++++++++++

\#Establish day

Weekday <- TempTestData..\$Weekday[TempTestData..\$Date\%in\%day]

\#Establish season

season <- TempTestData..\$Season[TempTestData..\$Date\%in\%day]

\#Raw

\#Do Am

AmResult <-

round(sum(TempTestData..[TempTestData..\$Date\%in\%day,][8:9]) /

TotalAmFactors.[type],0)

AmTotalResults..DayClSe[tl,season, Weekday] <- AmResult

\#Do Pm 
PmResult <-

round(sum(TempTestData..[TempTestData..\$Date\%in\%day,][17:18]) /

TotalPmFactors.[type],0)

PmTotalResults..DayCISe[tl,season,Weekday] <- PmResult

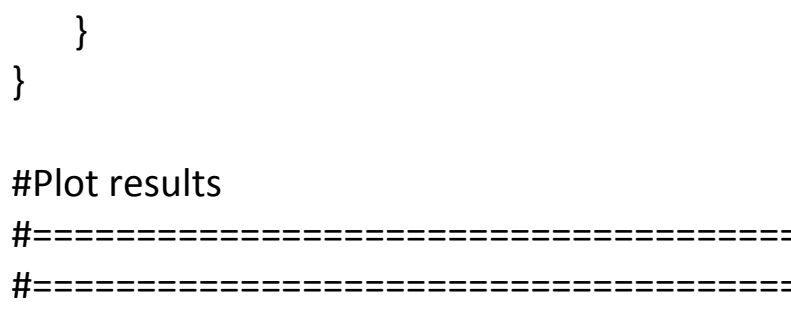

\#Create vector of II result matrices

Results. <- c("Am Study Area","Pm Study Area")

for(result in Results.)\{

if(result == "Am Study Area")\{Data.. <- AmTotalResults..DayClSe\}

if(result == "Pm Study Area")\{Data.. <- PmTotalResults..DayClSe $\}$

if(result == "Am NBPDP")\{Data.. <- AmNBPDPResults..DayClSe $\}$

if(result == "Pm NBPDP")\{Data.. <- PmNBPDPResults..DayClSe $\}$

Obs_<- list()

Est_<- list()

\#Local Total AM

\#+++++++++++++++++++++++

for(t $\mathrm{t}$ in TestLocations. $)\{$

for(season in c("Summer","Fall","Winter"))\{

for(weekday in c("Tuesday","Thursday"))\{

\#Determine symbol for plot

BikeFac <- CountLocationInformation..\$FactorGroup[match(tl, CountLocationInformation..\$Location)]

if(BikeFac $==$ "Path-Rec") $\{$ Pch $<-5\}$

if(BikeFac $==$ "Path-Commute") $\{$ Pch $<-25\}$

if(BikeFac == "Lane") $\{$ Pch $<-12\}$

if(BikeFac == "Blvd") $\{$ Pch $<-13\}$

if(BikeFac == "NoFacilities") $\{$ Pch $<-8\}$ 


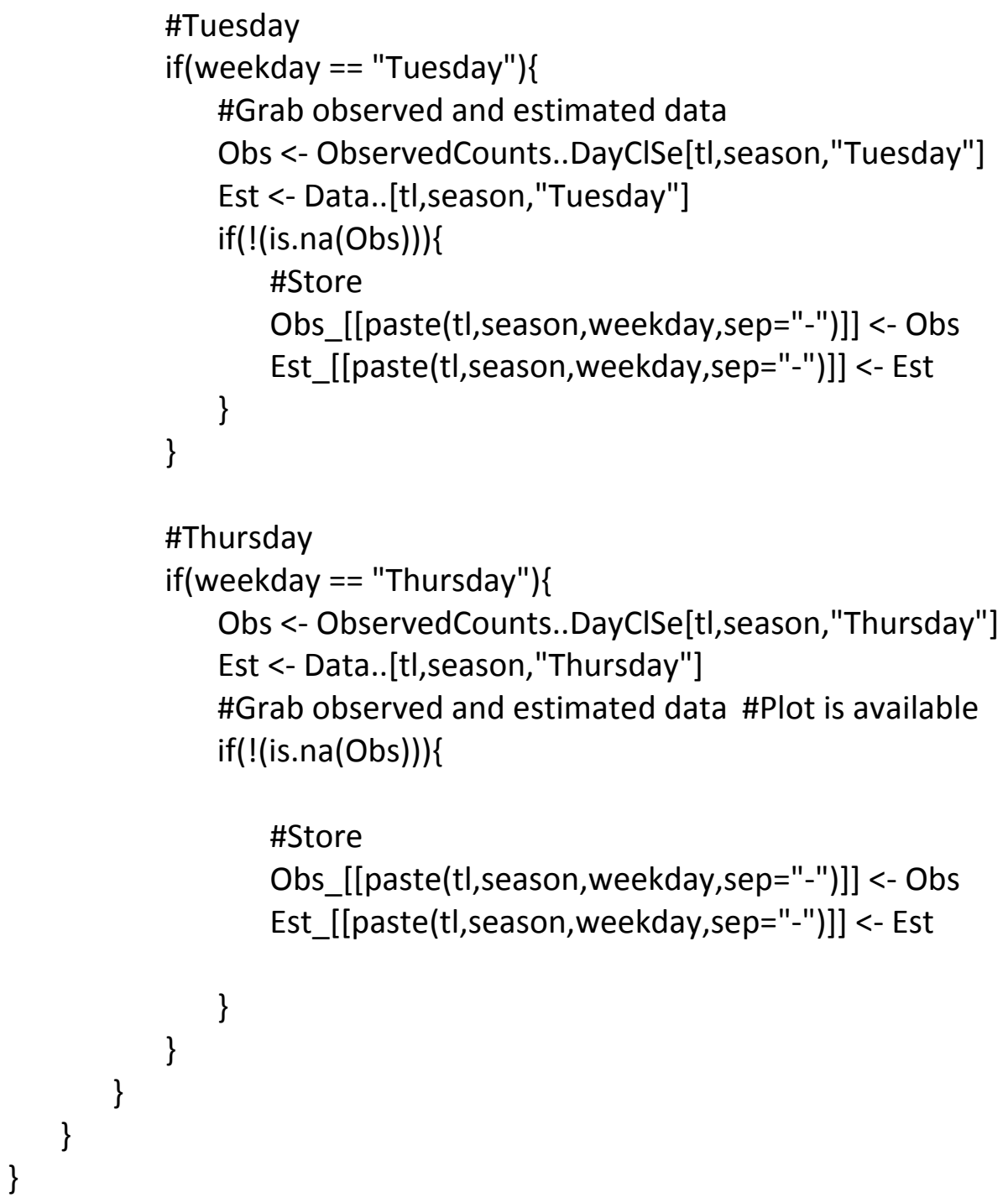


BestResult..\$FactorGroup <-

CountLocationInformation..\$FactorGroup[match(BestResult..\$Location, CountLocationInformation..\$Location)]

BestResult..\$FedClass <-

CountLocationInformation..\$FedClass[match(BestResult..\$Location,

CountLocationInformation..\$Location)]

BestResult..\$Locationld <-

CountLocationInformation..\$Locationld[match(BestResult..\$Location,

CountLocationInformation..\$Location)]

BestResult.. <- BestResult..[order(BestResult..\$AbsPctDiff),]

\#Result_[[result]] <- BestResult..

\#Add legend

RMSE <- round(summary(Im(unlist(Obs_) unlist(Est_)))\$sigma,2)

RMSE <- paste("RMSE = ",RMSE, sep="")

RSquared <- round(summary(Im(unlist(Obs_) unlist(Est_)))\$r.squared,2)

RSquared <- paste("R-squared = ", RSquared, sep= "")

MeanAbsDiff <- round(mean(BestResult..\$AbsPctDiff),2)

MeanAbsDiff <- paste("Mean Abs Diff = ", MeanAbsDiff)

MeanAbsDiff.Fg <-

round(tapply(BestResult..\$AbsPctDiff,BestResult..\$FactorGroup,mean),2)

if(result != "Am Study Area")\{

if(any(MeanAbsDiff.Fg < .30)) \{

ToSave <- MeanAbsDiff.Fg[MeanAbsDiff.Fg < .30]

Result2_[[paste(as.character(samplefactor), result,sep="-")]] <- ToSave \}

\}

\#End result loop

\}

\}

\#.

\#Apply Scenario 2 factors - analysis and comparison with NBPDP

\#.......

\#Create data to apply data 
TestData.. <- Hourly..

\#Define factors derived froim iterative process

TotalAmFactors. <- c(0.15,0.15,0.13,0.13,0.15)

names(TotalAmFactors.) <- c("Blvd","Lane","NoFacility","Path-Commute","PathRec")

TotalPmFactors. <- c(0.19,0.17,0.19,0.21,0.25)

names(TotalPmFactors.) <- c("Blvd","Lane","NoFacility","Path-Commute","PathRec")

\#define NPBDP factors

NBPDPAmFactors. <- c(0.10,0.10,0.10,0.10,0.10)

names(NBPDPAmFactors.) <- c("Blvd","Lane","NoFacility", "Path-Commute", "PathRec")

NBPDPPmFactors. <- $c(.15, .15, .15, .15, .15)$

names(NBPDPPmFactors.) <- c("Blvd","Lane", "NoFacility","Path-Commute","PathRec")

\#Apply Factors to count stations not selected for factor creation

$\#$.

\#Determine count stations to apply factors too

\#TestData.. <- Hourly..[!(Hourly..\$Location\%in\%Samplelds.),]

\#Create vector of test locations

TestLocations. <- unique(TestData..\$Location)

\#Remove All days but Tuesday and Thursday

TestData.. <- TestData..[TestData..\$Weekday\%in\%c("Tuesday","Thursday"),]

\#Remove Spring

TestData.. <- TestData..[TestData..\$Season != "Spring",]

\#Append factor Groups

TestData..\$FactorGroup <-

CountLocationInformation..\$FactorGroup[match(TestData..\$Location,

CountLocationInformation..\$Location)]

\#Create an observed counts array

$\#-$

\#Create an array to store observed counts

ObservedCounts..DayClSe <- $\operatorname{array}(\mathrm{NA}, \mathrm{c}$ (length(TestLocations.), 3, 2), dimnames = list(TestLocations., c("Summer","Fall","Winter"),

c("Tuesday","Thursday")))

\#Populate array with observed values 


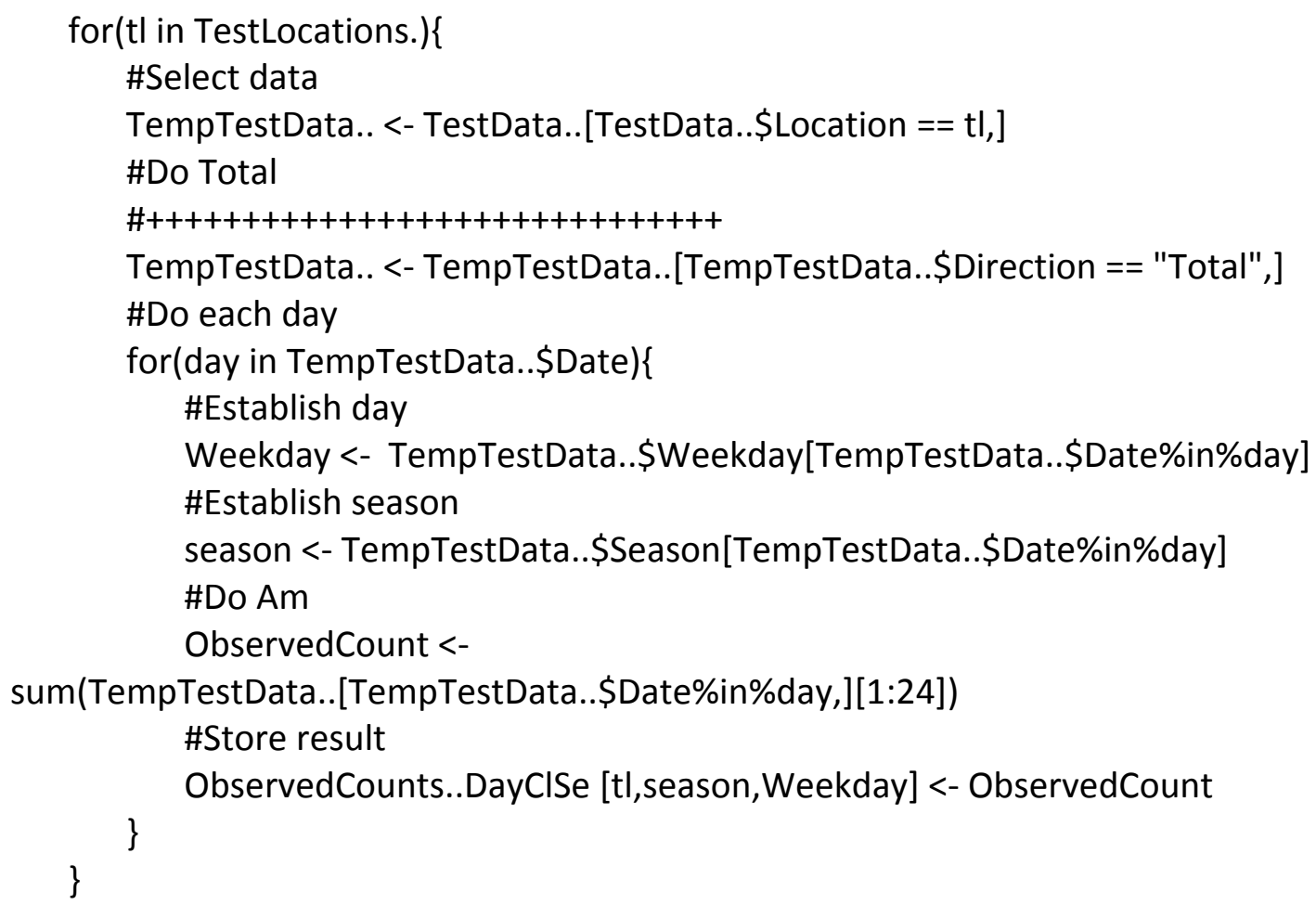

PmTotalResults..DayCISe <- array(NA, c(length(TestLocations.), 3, 2), dimnames = list(TestLocations., c("Summer","Fall","Winter"),

AmNBPDPResults..DayClSe <- array(NA, c(length(TestLocations.), 3, 2), dimnames = list(TestLocations., c("Summer","Fall","Winter"),

c("Tuesday","Thursday")))

PmNBPDPResults..DayClSe <- $\operatorname{array}(N A, c($ length(TestLocations.), 3, 2), dimnames = list(TestLocations., c("Summer","Fall","Winter"),

c("Tuesday","Thursday")))

\#Apply Factors 


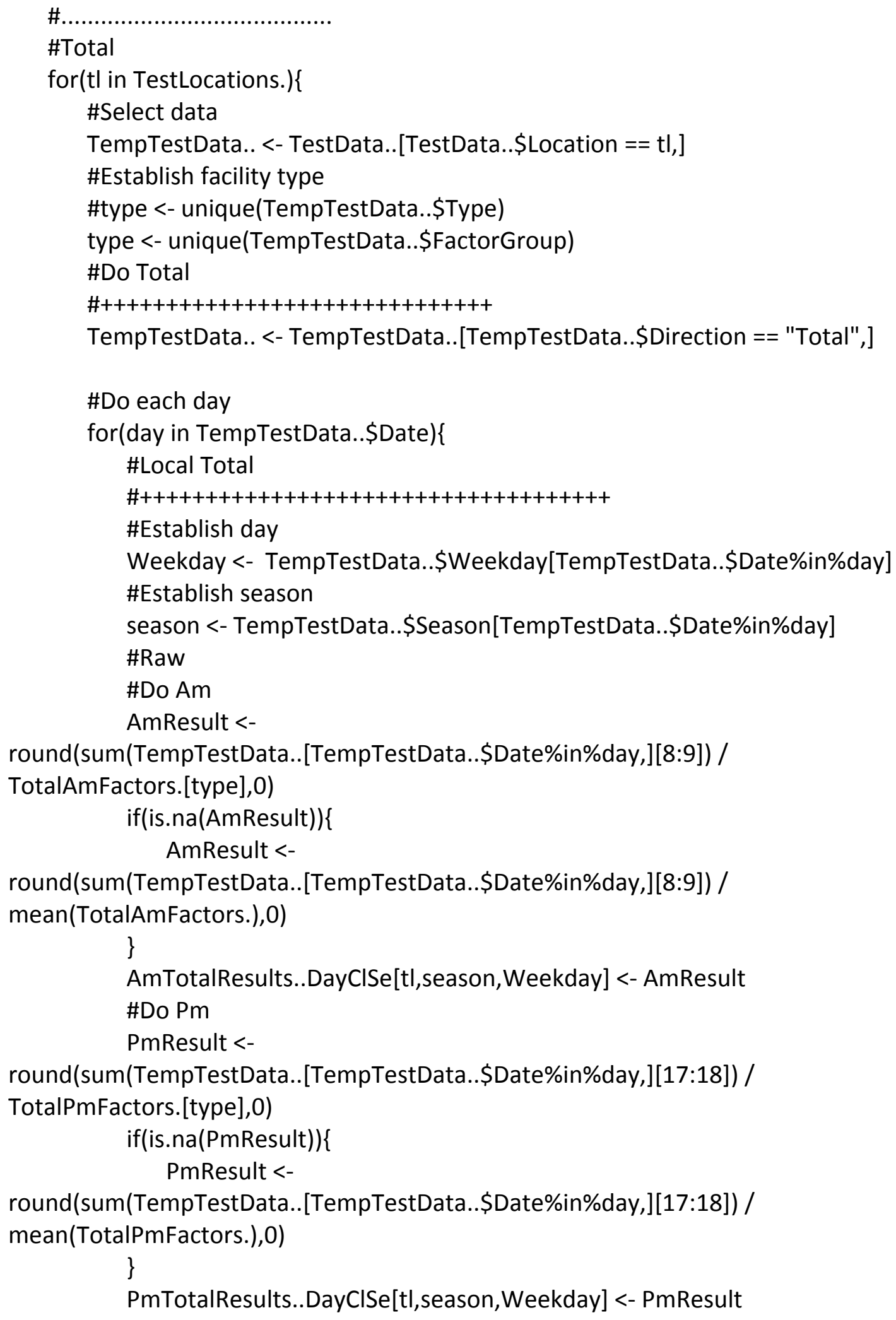




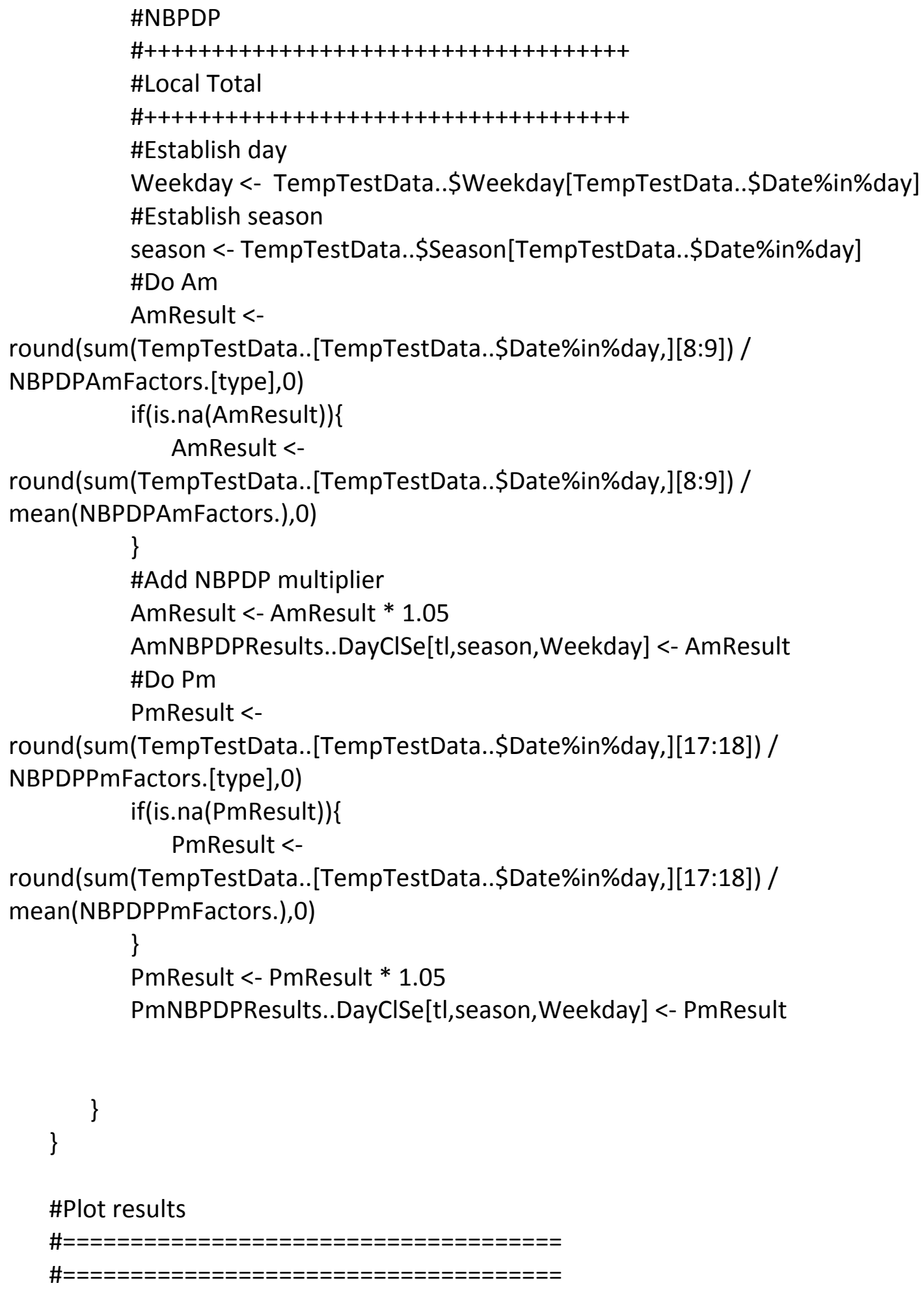


pdf(paste("Reports/FactorReports/SelectedFactors_ComarisonTotal_and_NBPDP.pd $f^{\prime \prime}$, sep $="$ "), width = 11.5, height = 9)

\#Set pdf parameters

$\operatorname{par}(\operatorname{mfrow}=c(2,2), \mathrm{xpd}=$ TRUE, $\operatorname{mar}=\operatorname{par}() \$ \operatorname{mar}+c(0,2,0,9))$

$X \lim <-c(0,3000)$

Ylim <- c(0,3000)

\#Create vector of II result matrices

Results. <- c("Am Study Area","Pm Study Area","Am NBPDP","Pm NBPDP")

Result_<- list()

for(result in Results.)\{

if(result == "Am Study Area")\{Data.. <- AmTotalResults..DayClSe $\}$

if(result == "Pm Study Area")\{Data.. <- PmTotalResults..DayClSe\}

if(result == "Am NBPDP")\{Data.. <- AmNBPDPResults..DayCISe $\}$

if(result == "Pm NBPDP")\{Data.. <- PmNBPDPResults..DayClSe $\}$

Obs_<- list()

Est_<- list()

\#Local Total AM

\#+++++++++++++++++++++++

for(tl in TestLocations. ) \{

for(season in c("Summer","Fall","Winter"))\{

for(weekday in c("Tuesday","Thursday"))\{

\#Determine symbol for plot

BikeFac <- CountLocationInformation..\$FactorGroup[match(tl,

CountLocationInformation..\$Location)]

if(BikeFac $==$ "Path-Rec") $\{$ Pch $<-5\}$

if(BikeFac $==$ "Path-Commute") $\{$ Pch $<-25\}$

if(BikeFac == "Lane") $\{$ Pch <- 12\}

if(BikeFac $==$ "Blvd") $\{$ Pch $<-13\}$

if(BikeFac $==$ "NoFacilities") $\{$ Pch $<-8\}$

\#Tuesday

if(weekday == "Tuesday")\{

\#Grab observed and estimated data

Obs <- ObservedCounts..DayClSe[tl,season,"Tuesday"]

Est <- Data... [tl,season,"Tuesday"]

if(!(is.na(Obs)))\{ 


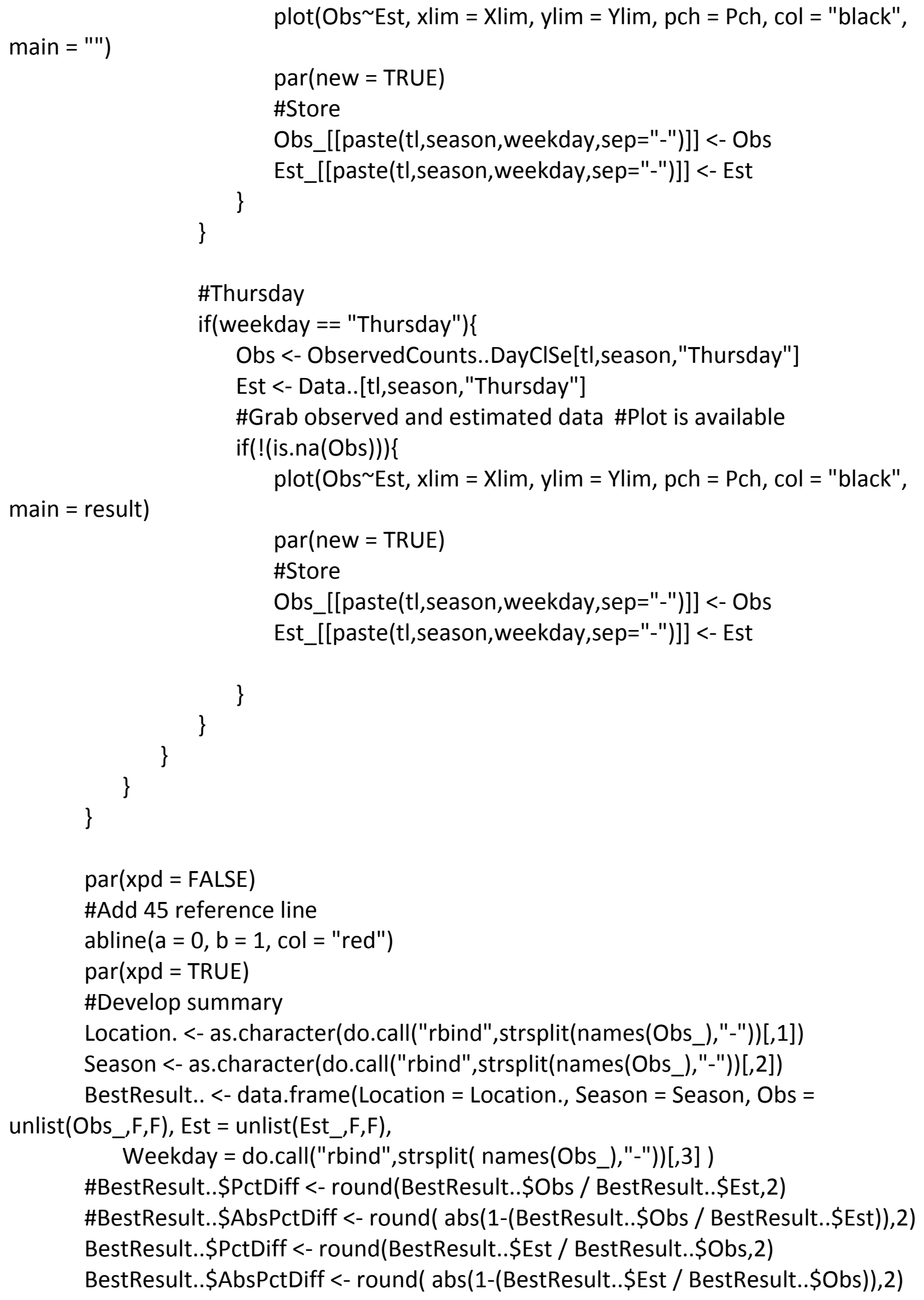


BestResult..\$Type <-

CountLocationInformation..\$Type[match(BestResult..\$Location,

CountLocationInformation..\$Location)]

BestResult..\$FactorGroup <-

CountLocationInformation..\$FactorGroup[match(BestResult..\$Location,

CountLocationInformation..\$Location)]

BestResult..\$FedClass <-

CountLocationInformation..\$FedClass[match(BestResult..\$Location,

CountLocationInformation..\$Location)]

BestResult..\$Locationld <-

CountLocationInformation..\$Locationld[match(BestResult..\$Location,

CountLocationInformation..\$Location)]

BestResult.. <- BestResult..[order(BestResult..\$AbsPctDiff),]

Result_[[result]] <- BestResult..

\#Add legend

RMSE <- round(summary(Im(unlist(Obs_) unlist(Est_)))\$sigma,2)

RMSE <- paste("RMSE = ",RMSE,sep="")

RSquared <- round(summary(Im(unlist(Obs_) unlist(Est_)))\$r.squared,2)

RSquared <- paste("R-squared = ", RSquared, sep= "")

MeanAbsDiff <- round(mean(BestResult..\$AbsPctDiff),2)

MeanAbsDiff <- paste("Mean Abs Diff = ", MeanAbsDiff)

MeanAbsDiff.Fg <-

round(tapply(BestResult..\$AbsPctDiff,BestResult..\$FactorGroup,mean),2)

\#Add legend 1

legend("topleft",title = "Results Summary", legend =c(RMSE, RSquared, MeanAbsDiff) )

\#Add legend 2

legend(3150,3100,title = "Mean Abs Diff by Factor Group", legend $=c$ (paste(names(MeanAbsDiff.Fg[1]),MeanAbsDiff.Fg[1],sep="-"), paste(names(MeanAbsDiff.Fg[2]),MeanAbsDiff.Fg[2],sep=""),paste(names(MeanAbsDiff.Fg[3]),MeanAbsDiff.Fg[3],sep="-"), paste(names(MeanAbsDiff.Fg[4]),MeanAbsDiff.Fg[4],sep="-

"),paste(names(MeanAbsDiff.Fg[5]),MeanAbsDiff.Fg[5],sep="-")),cex = .9)

\#Add legend 3

if(result == "Am Study Area")\{AmTotalFactors. <- TotalAmFactors. $\}$

if(result == "Pm Study Area")\{AmTotalFactors. <- TotalPmFactors. $\}$ 
if(result == "Am NBPDP")\{AmTotalFactors. <- NBPDPAmFactors. $\}$

if(result == "Pm NBPDP")\{AmTotalFactors. <- NBPDPPmFactors. $\}$

AmTotalFactors.<-

paste(names(AmTotalFactors.), round(AmTotalFactors.,2), sep="-")

AmNBPDPFactors. <- NBPDPAmFactors.

AmNBPDPFactors.<- paste(names(AmNBPDPFactors.),AmNBPDPFactors.,sep="-")

\#legend("bottomright", legend = c("Factors","Local",AmTotalFactors.))

legend $(3150,2000$, legend = c("Factors", "Local",AmTotalFactors.))

\#Add legend 4

legend("bottomright",title = "Legend", legend = c("Path-Rec","Path-

Commute","Lane","Blvd","NoFacilities"),

pch $=c(5,25,12,13,8)$, cex $=.8)$

$\operatorname{par}(\mathrm{xpd}=\mathrm{FALSE})$

\#End result loop

\}

\#Close pdf

dev.off() 